\title{
Nucleophilicities of Primary and Secondary Amines
}

\author{
Frank Brotzel, Ying Cheung Chu, and Herbert Mayr* \\ Department Chemie und Biochemie, Ludwig-Maximilians-Universität München, \\ Butenandtstr. 5-13 (Haus F), D-81377 München, Germany
}

\author{
Herbert.Mayr@cup.uni-muenchen.de
}

\section{Content}

1. General 3

2. Correlation with literature data $\quad 4$

3. Ammonia (1a) 8

$\begin{array}{ll}\text { 4. } & \text { Methylamine (1b) } \\ \end{array}$

$\begin{array}{ll}\text { 5. Ethylamine (1c) } & 16\end{array}$

$\begin{array}{ll}\text { 6. Isopropylamine (1d) } & 18\end{array}$

$\begin{array}{lll}\text { 7. } & \text { tert-Butylamine (1e) } & 20\end{array}$

\begin{tabular}{ll} 
8. & Glycinenitrile (1f) \\
\hline
\end{tabular}

$\begin{array}{ll}\text { 9. } & \text { Methyl glycinate (1g) }\end{array}$

10. Glycinamide (1h) 29

11. 2-Aminoethanol (1i) 33

12. 1,2-Ethanediamine (1j) 37 
13. 1,3-Propanediamine (1k)

14. Propargylamine (1I)

15. Allylamine (1m)

48

16. Benzylamine (1n)

17. 4-Chloroaniline (10)

55

18. Aniline (1p)

19. $p$-Toluidine (1q)

62

20. p-Anisidine (1r)

66

21. Dimethylamine (1s)

69

22. Diethylamine (1t)

73

23. Methylaminoacetonitrile (1u)

77

24. 2,2'-Iminodiethanol (1v)

79

25. Pyrrolidine (1w)

81

26. Piperidine (1x)

86

27. Perhydroazepine (1y)

89

28. Morpholine (1z)

93

29. Piperazine (1zz)

98

30. Literature

102 


\section{General}

1.1. Determination of rate constants in water

When an amine is dissolved in water, the concentration of hydroxide increases by protolysis. For that reason we have to calculate the concentration of the free amines $[\mathrm{Nu}]_{\text {eff }}$ and of hydroxide $\left[\mathrm{OH}^{-}\right]$with the $\mathrm{p} K_{\mathrm{B}}$ of the amines. The $\mathrm{p} K_{\mathrm{B}}$ values of the amines are taken from ref 1 unless otherwise stated.

$$
\begin{aligned}
& \mathrm{R}_{2} \mathrm{NH}+\mathrm{H}_{2} \mathrm{O} \rightleftharpoons \mathrm{R}_{2} \stackrel{+}{\mathrm{N}_{2}}+{ }^{-} \mathrm{OH} \\
& K_{B}=\frac{[\text { ammonium }]\left[\mathrm{OH}^{-}\right]}{[\text {amine }]_{e f f}} \\
& {[\text { amine }]_{0}=[\text { amine }]_{\text {eff }}+[\text { ammonium }]=[\text { amine }]_{\text {eff }}+\left[\mathrm{OH}^{-}\right]}
\end{aligned}
$$

(2) in (1)

$$
K_{B}=\frac{\left[\mathrm{OH}^{-}\right]^{2}}{[\text { amine }]_{0}-\left[\mathrm{OH}^{-}\right]}
$$

Solving of the quadratic equation (3) leads to one logic solution for $[\mathrm{OH}]$ (The one with the "+" in the numerator).

$$
\left[\mathrm{OH}^{-}\right]=-\frac{K_{B}}{2}+\sqrt{\left(\frac{K_{B}}{2}\right)^{2}+K_{B}[\text { amine }]_{0}}
$$

The rates of the combination reactions were determined by mixing the colored aqueous solutions of the benzhydrylium salts with aqueous solutions of the amines (usually $>10$ equivalents). Because the products are colorless, the rates of the reactions were determined by UV-Vis spectroscopic monitoring of the absorbances with time.

$$
-\frac{d\left[\mathrm{R}^{+}\right]}{d t}=k_{\mathrm{obs}}\left[\mathrm{R}^{+}\right]
$$

The consumption of the benzhydrylium cations may be due to the reaction with the amines, hydroxide ions and the solvent water.

$$
\begin{aligned}
k_{\mathrm{obs}} & =k_{2, \mathrm{~N}}[\text { amine }]_{\mathrm{eff}}+k_{2, \mathrm{OH}-}\left[\mathrm{OH}^{-}\right]+k_{\mathrm{W}}+k_{-1} \\
& \left.=k_{1 \Psi}+k_{2, \mathrm{OH}-}\left[\mathrm{OH}^{-}\right] \text {, with } k_{1 \Psi}=k_{2, \mathrm{~N}} \text { [amine }\right]_{\mathrm{eff}}+k_{\mathrm{W}}+k_{-1} \text {, and }\left[\mathrm{OH}^{-}\right] \text {from eq. (4) }
\end{aligned}
$$

The amines are usually used in more than 10 -fold excess over the benzhydrylium cations in order to arrive at pseudo first-order conditions. The concentrations of the amines as well as that of hydroxide therefore remains constant during the reactions. With the already published second-order rate 
constants $k_{2, \mathrm{OH}}$-for the reactions of hydroxide with benzhydrylium ions and the first-order rate constants $k_{w}$ for the reactions of water with benzhydrylium ions, ${ }^{2}$ we get the second-order rate constants for the reactions of the amines with the benzhydrylium ions $k_{2, N}$ from a plot of $k_{1 \Psi}$ versus [amine $]_{\text {eff }}$.

\subsection{Determination of the equilibrium constants in water}

Scheme 1

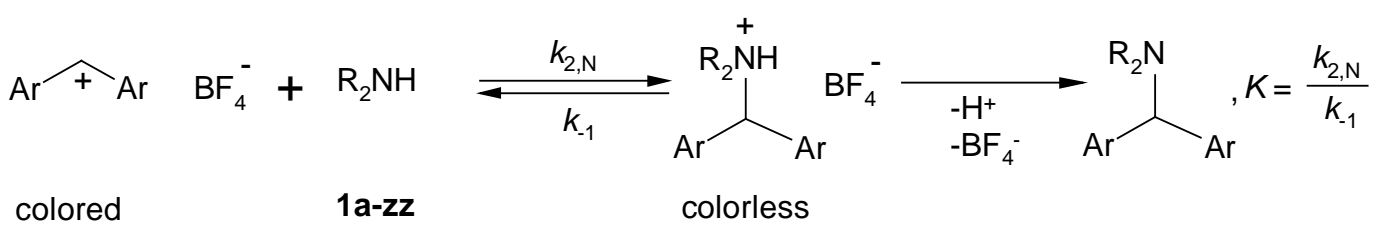

We were not able to measure the equilibrium constants $K$ (Scheme 1$)$ in water directly. When an aqueous solution of the benzhydrylium salt was combined with the amines $\mathbf{1 f}, \mathbf{1 p}, \mathbf{1 q}$ or $\mathbf{1 r}$, the UV/Vis spectrum for the benzhydrylium ion did not show a constant end absorption because of the slow reactions of the benzhydrylium ions with hydroxide and water in the presence of amine. Equilibrium constants were, therefore calculated from the ratio of the second order rate constants $k_{2, N}$ for the forward reaction and the first order rate constant $k_{-1}$ for the backward reaction. The thus obtained equilibrium constants are less accurate than the directly measured ones and are, therefore, written in parentheses.

\section{Correlation with literature data}

2.1. Nucleophilic reactivity of amines toward ethylene oxide

Virtanen and Korhonen studied the kinetics of the reaction of ethylene oxide with a series of nucleophiles in aqueous solution at $25^{\circ} \mathrm{C}$ (Scheme 2). Figure 1 shows a correlation between the reactivities of nucleophiles toward ethylene oxide 2 and the reactivities of the nucleophiles determined toward benzhydrylium ions.

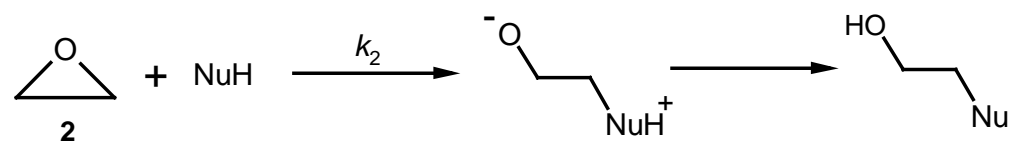

Scheme 2 


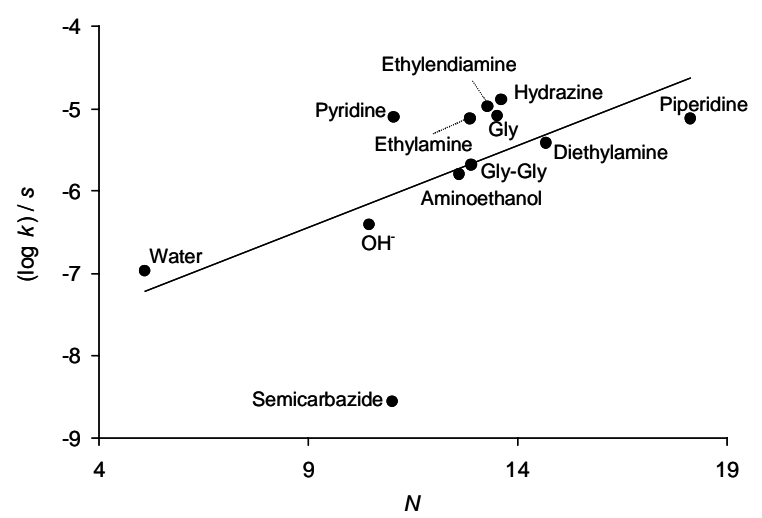

Figure 1. Relationship between the rate constants for the reactions of various nucleophiles with ethylene oxide 2 in water at $25^{\circ} \mathrm{C}$ (rate constants $k$ from ref 3 ; the rate constant for water is first-order, all others are second-order) with the $N$ and s parameters (determined at $20^{\circ} \mathrm{C}$ in this work and in refs 2, 4, 5; correlation line shown includes all data $(\log k) / s=0.199 N-8.23 ; R^{2}=0.3147$; without semicarbazide $\left.(\log k) / s=0.160 N-7.52 ; R^{2}=0.5821\right)$.

\subsection{Nucleophilic reactivity of amines toward a $\mathrm{N}$-nitrososulfonamide}

Leis investigated the kinetics of the reaction of $N$-methyl- $N$-nitrosotoluene- $p$-sulfonamide $\mathbf{3}$ with several primary, secondary and tertiary amines in aqueous solution at $25^{\circ} \mathrm{C}$ (Scheme 3). ${ }^{6}$ Figure 2 shows a moderate correlation between reactivities of amines toward the nitrogen electrophile $\mathbf{3}$ and toward benzhydrylium ions.

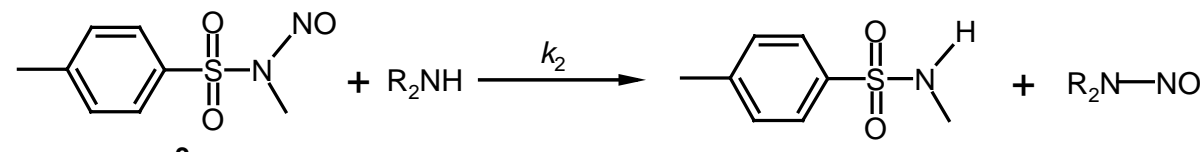

3

Scheme 3

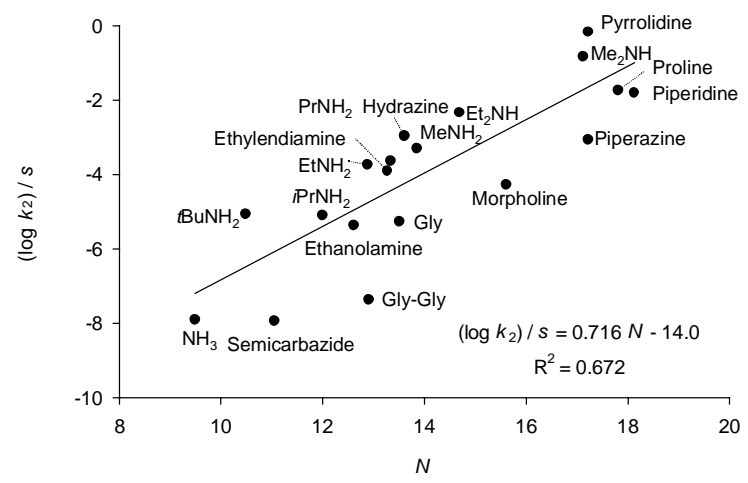

Figure 2. Relationship between the rate constants for the reactions of various amines with $N$ nitrososulfonamide 3 in water at $25^{\circ} \mathrm{C}$ (second-order rate constants $k_{2}$ from ref 6 ) with the $N$ and $\mathrm{s}$ parameters (determined at $20^{\circ} \mathrm{C}$ in this work and in refs 2,7 ). 


\subsection{Nucleophilic reactivity of amines toward S-nitrosopenicillamine}

Williams reported on the kinetics of the reaction of $S$-nitrosopenicillamine 4 with various amines in aqueous solution at $25{ }^{\circ} \mathrm{C}$ (Scheme 4). ${ }^{8}$ Figure 3 shows no correlation between the reactivities of amines determined toward $\mathbf{4}$ and the reactivities of amines determined toward benzhydrylium ions.

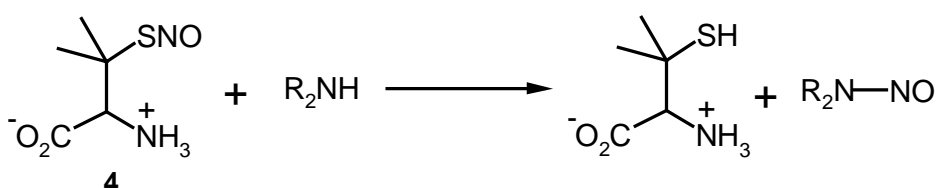

Scheme 4

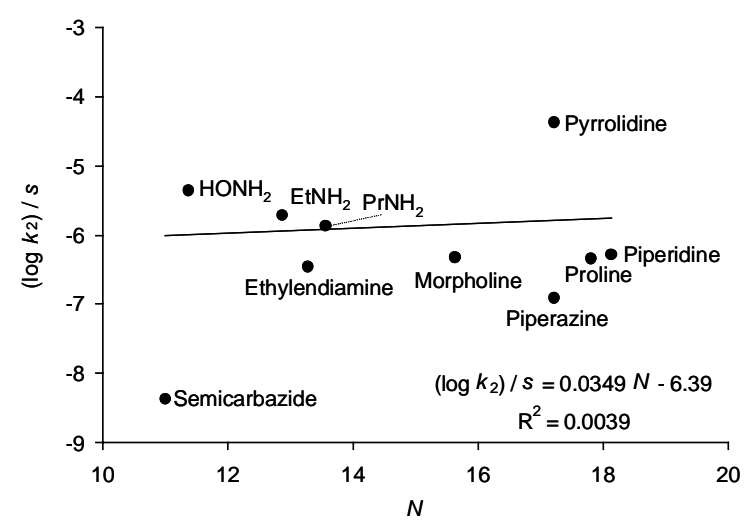

Figure 3. Relationship between the rate constants for the reactions of various amines with $S$ nitrosopenicillamine 4 in water at $25^{\circ} \mathrm{C}$ (second-order rate constants $k_{2}$ from ref 8) with the $\mathrm{N}$ and $\mathrm{s}$ parameters (determined at $20^{\circ} \mathrm{C}$ in this work and in refs 2,7 ).

\subsection{Nucleophilic reactivity of amines toward benzoylacetylene}

Um investigated the kinetics of the reactions of 1-phenyl-2-propyn-1-one 5 with primary amines in aqueous solution at $25^{\circ} \mathrm{C}$ (Scheme 5). ${ }^{9}$ Figure 4 shows a poor correlation between the two data sets. Because of the narrow reactivity range investigated, the slope of this correlation cannot be interpreted.

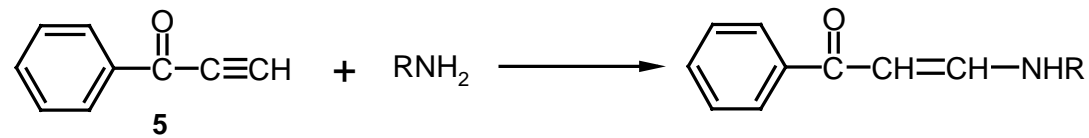

Scheme 5 


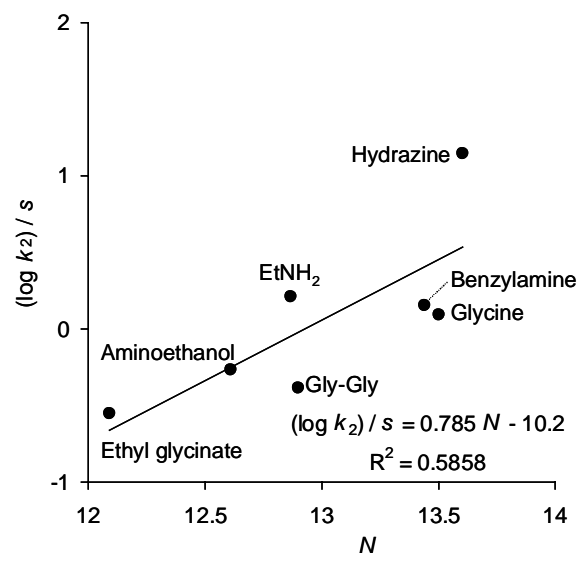

Figure 4. Relationship between the rate constants for the reactions of primary amines with 1-phenyl-2propyn-1-one 5 in water at $25^{\circ} \mathrm{C}$ (second-order rate constants $k_{2}$ from ref 9 ) with the $N$ and $s$ parameters (determined at $20^{\circ} \mathrm{C}$ in this work and ref 7 ; the $N$ parameter for ethyl glycinate was assumed to be the same as for methyl glycinate). 


\section{Ammonia (1a)}

3.1. Rate constants in water

3.1.1. Reaction of Ammonia (1a) with (mor) ${ }_{2} \mathrm{CH}^{+} \mathrm{BF}_{4}{ }_{4}$ (at $20^{\circ} \mathrm{C}$, cosolvent: 9 vol- $\% \mathrm{CH}_{3} \mathrm{CN}$, stoppedflow, detection at $607 \mathrm{~nm}$ )

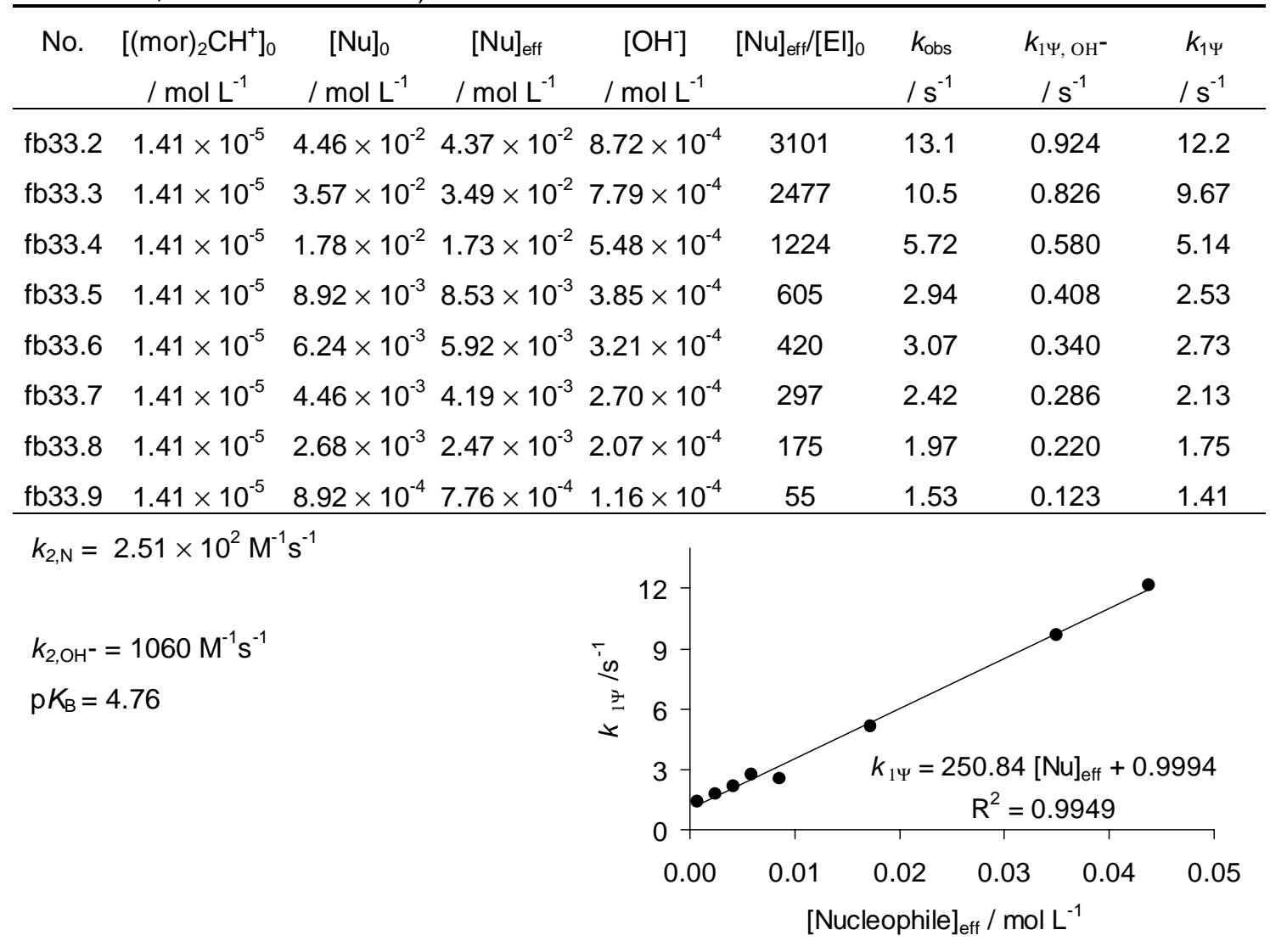


3.1.2. Reaction of Ammonia (1a) with $(\mathrm{dma})_{2} \mathrm{CH}^{+} \mathrm{BF}_{4}^{-}$(at $20{ }^{\circ} \mathrm{C}$, cosolvent: 0.4 vol- $\% \mathrm{CH}_{3} \mathrm{CN}$, stopped-flow, detection at $613 \mathrm{~nm}$ )

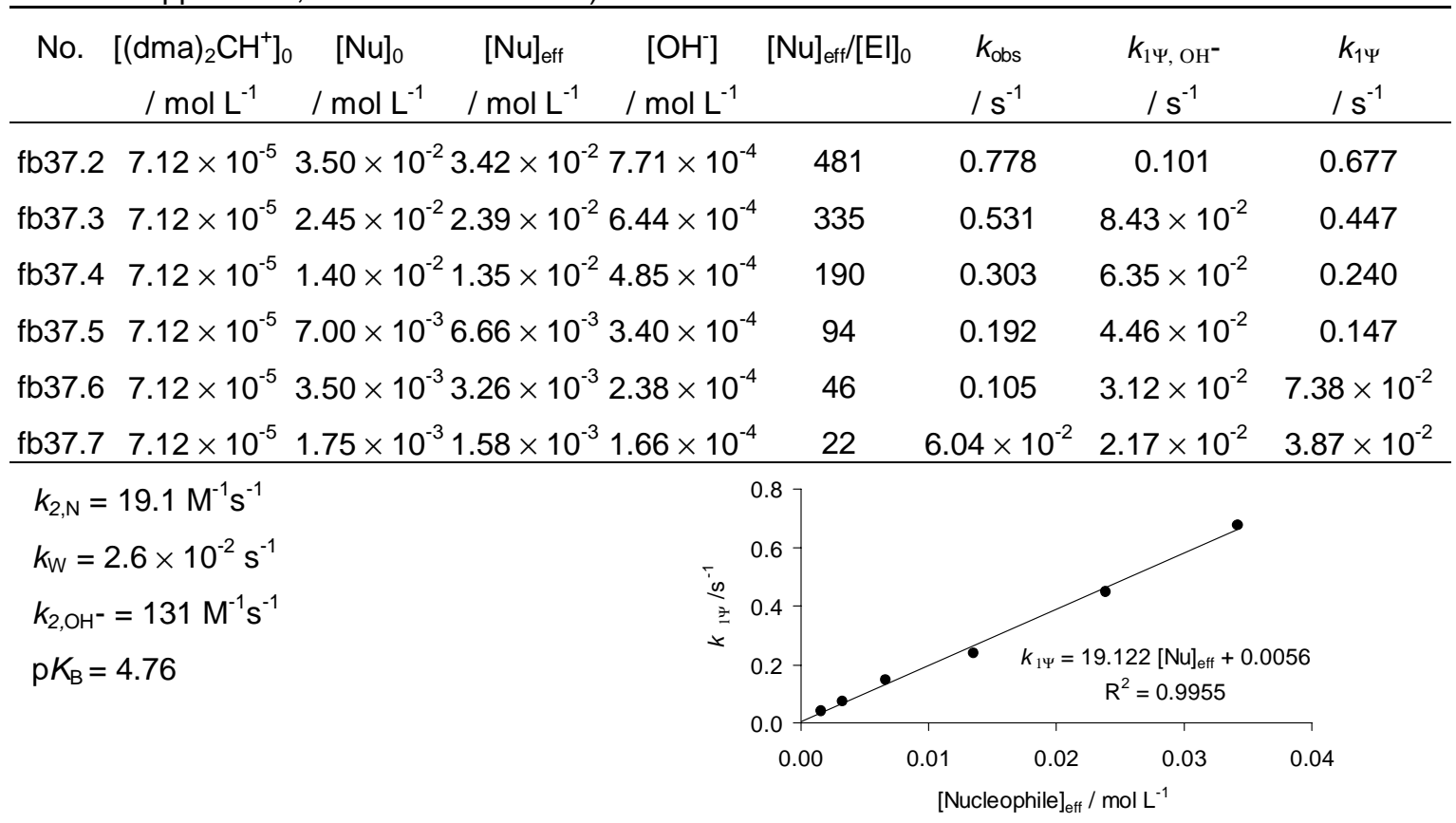

3.1.3. Reaction of Ammonia (1a) with (pyr) $)_{2} \mathrm{CH}^{+} \mathrm{BF}_{4}^{-}$: (at $20^{\circ} \mathrm{C}$, cosolvent: 0.4 vol-\% $\mathrm{CH}_{3} \mathrm{CN}, \mathrm{J} \& \mathrm{M}$, detection at $611 \mathrm{~nm}$ )

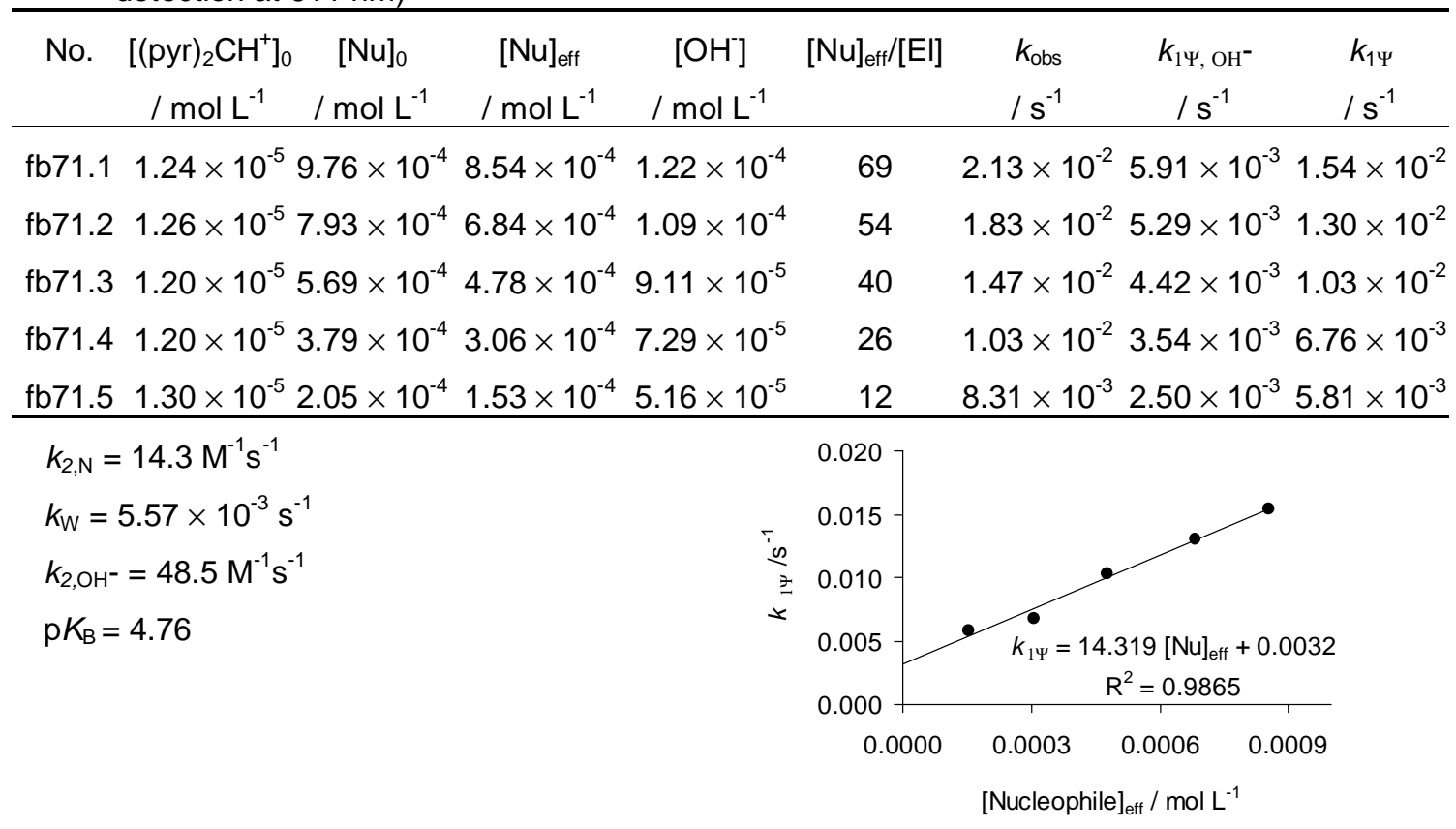


3.1.4. Reaction of Ammonia (1a) with (thq) ${ }_{2} \mathrm{CH}^{+} \mathrm{BF}_{4}$ : (at $20^{\circ} \mathrm{C}$, cosolvent: 0.4 vol- $\% \mathrm{CH}_{3} \mathrm{CN}, \mathrm{J} \& \mathrm{M}$, detection at $618 \mathrm{~nm})$

\begin{tabular}{|c|c|c|c|c|c|c|c|c|}
\hline No. & $\begin{array}{c}{\left[(\text { thq })_{2} \mathrm{CH}^{+}\right]_{0}} \\
/ \mathrm{mol} \mathrm{L}^{-1}\end{array}$ & $\begin{array}{c}{[\mathrm{Nu}]_{0}} \\
/ \mathrm{mol} \mathrm{L}^{-1}\end{array}$ & $\begin{array}{c}{[\mathrm{Nu}]_{\mathrm{eff}}} \\
/ \mathrm{mol} \mathrm{L}^{-1}\end{array}$ & $\begin{array}{c}{\left[\mathrm{OH}^{-}\right]} \\
/ \mathrm{mol} \mathrm{L}^{-1}\end{array}$ & {$[\mathrm{Nu}]_{\mathrm{eff}} /[\mathrm{El}]_{0}$} & $\begin{array}{l}k_{\mathrm{obs}} \\
/ \mathrm{s}^{-1}\end{array}$ & $\begin{array}{c}k_{1 \Psi, \mathrm{OH}^{-}} \\
/ \mathrm{s}^{-1} \\
\end{array}$ & $\begin{array}{l}k_{1 \Psi} \\
/ s^{-1}\end{array}$ \\
\hline $\mathrm{fb} 69.4$ & $1.62 \times 10^{-6}$ & $1.98 \times 10^{-3}$ & $1.80 \times 10^{-3}$ & $1.77 \times 10^{-4}$ & 1113 & $1.53 \times 10^{-2}$ & $4.18 \times 10^{-3}$ & $1.11 \times 10^{-2}$ \\
\hline fb69.5 & $1.57 \times 10^{-6}$ & $1.21 \times 10^{-3}$ & $1.07 \times 10^{-3}$ & $1.37 \times 10^{-4}$ & 684 & $1.11 \times 10^{-2}$ & $3.22 \times 10^{-3}$ & $7.88 \times 10^{-3}$ \\
\hline fb69.6 & $1.63 \times 10^{-6}$ & $3.99 \times 10^{-4}$ & $3.24 \times 10^{-4}$ & $7.50 \times 10^{-5}$ & 199 & $5.21 \times 10^{-3}$ & $1.77 \times 10^{-3}$ & $3.44 \times 10^{-3}$ \\
\hline $\mathrm{fb} 69.7$ & $1.64 \times 10^{-6}$ & $8.01 \times 10^{-5}$ & $5.05 \times 10^{-5}$ & $2.96 \times 10^{-5}$ & 31 & $2.35 \times 10^{-3}$ & $6.99 \times 10^{-4}$ & $1.65 \times 10^{-3}$ \\
\hline $\mathrm{fb} 69.8$ & $4.14 \times 10^{-6}$ & $1.48 \times 10^{-4}$ & $1.05 \times 10^{-4}$ & $4.28 \times 10^{-5}$ & 25 & $3.01 \times 10^{-3}$ & $1.01 \times 10^{-3}$ & $2.00 \times 10^{-3}$ \\
\hline fb69.9 & $4.18 \times 10^{-6}$ & $8.19 \times 10^{-5}$ & $5.19 \times 10^{-5}$ & $3.00 \times 10^{-5}$ & 12 & $2.81 \times 10^{-3}$ & $7.09 \times 10^{-4}$ & $2.10 \times 10^{-3}$ \\
\hline $\mathrm{fb} 69.10$ & $3.92 \times 10^{-6}$ & $3.08 \times 10^{-4}$ & $2.43 \times 10^{-4}$ & $6.50 \times 10^{-5}$ & 62 & $4.68 \times 10^{-3}$ & $1.53 \times 10^{-3}$ & $3.15 \times 10^{-3}$ \\
\hline \multicolumn{2}{|c|}{$k_{2, \mathrm{~N}}=5.39 \mathrm{M}^{-1} \mathrm{~s}^{-1}$} & & & & 0.012 & & & \\
\hline \multicolumn{2}{|c|}{$k_{\mathrm{w}}=2.20 \times 10^{-3} \mathrm{~s}^{-1}$} & & & & 0.009 & & & \\
\hline \multicolumn{2}{|c|}{$k_{2, \mathrm{OH}^{-}}=23.6 \mathrm{M}^{-1} \mathrm{~s}^{-1}$} & & & & 0.006 & & & \\
\hline \multirow[t]{2}{*}{$\mathrm{p} K_{\mathrm{B}}=$} & 4.76 & & & & $\begin{array}{l}0.003 \\
0.000\end{array}$ & & $R^{2}=0.9944$ & \\
\hline & & & & & 0.0000 & 0.0005 & $0010 \quad 0.0015$ & 0.0020 \\
\hline & & & & & & [Nucle & nol L-1 & \\
\hline
\end{tabular}

3.1.5. Reaction of Ammonia (1a) with (ind) ${ }_{2} \mathrm{CH}^{+} \mathrm{BF}_{4}{ }^{-}$: (at $20^{\circ} \mathrm{C}$, cosolvent: 0.4 vol-\% $\mathrm{CH}_{3} \mathrm{CN}, \mathrm{J} \& \mathrm{M}$, detection at $614 \mathrm{~nm}$ )

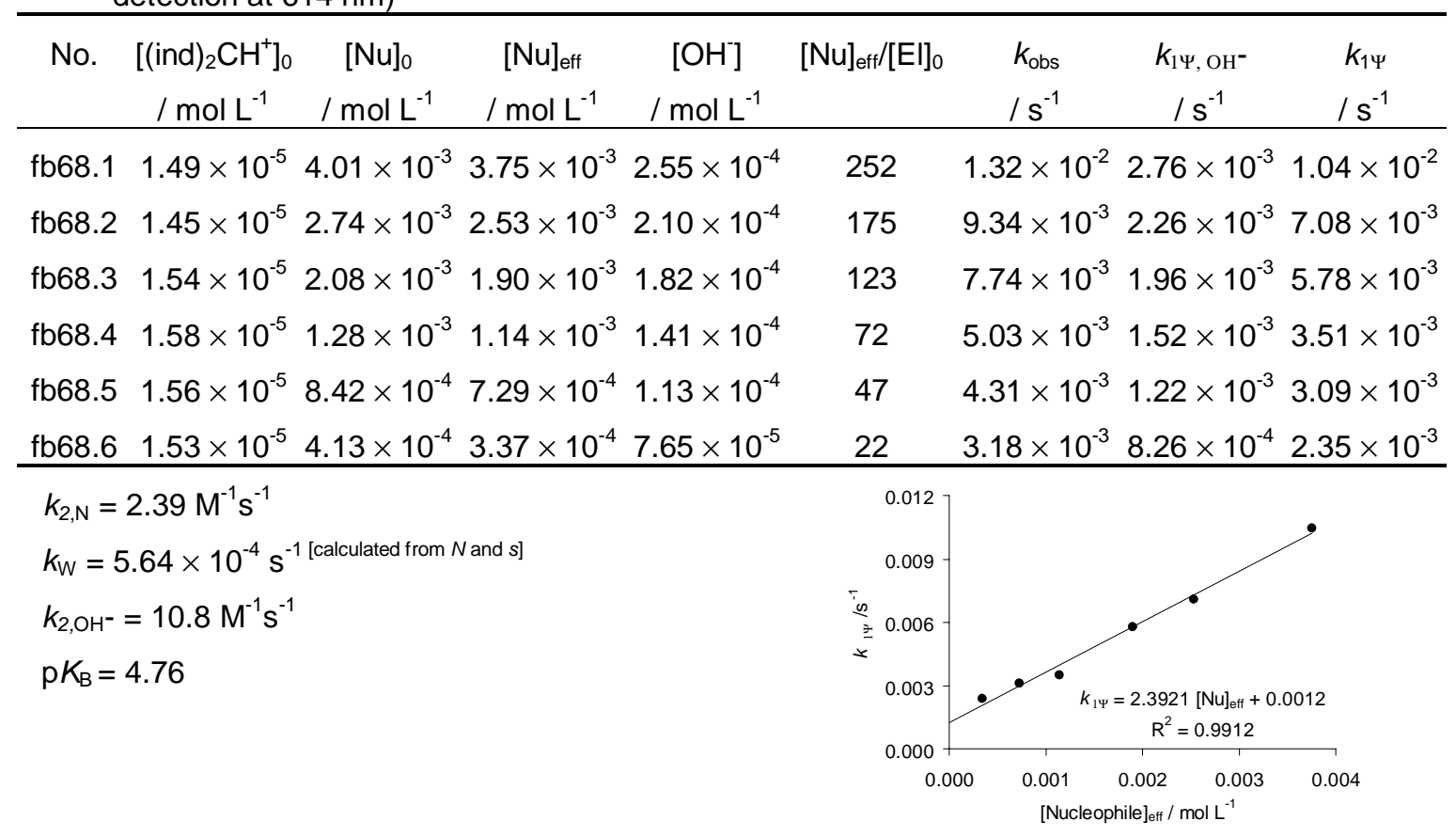


3.1.6. Reaction of Ammonia (1a) with (jul) ${ }_{2} \mathrm{CH}^{+} \mathrm{BF}_{4}$ : (at $20^{\circ} \mathrm{C}$, cosolvent: 0.4 vol- $\% \mathrm{CH}_{3} \mathrm{CN}$, stopped-flow, detection at $634 \mathrm{~nm}$ )

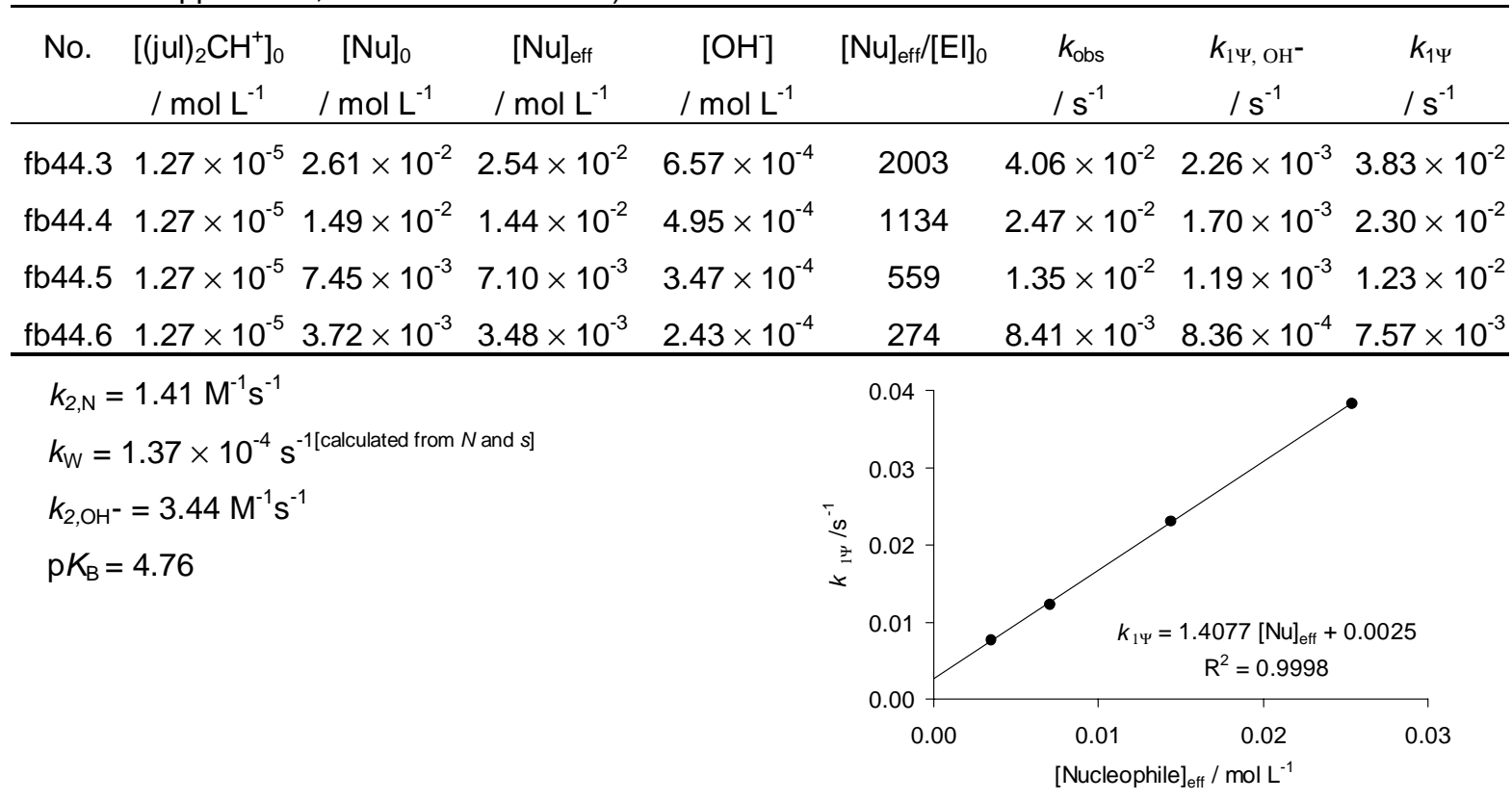

3.1.7. Reaction of Ammonia (1a) with (lil) ${ }_{2} \mathrm{CH}^{+} \mathrm{BF}_{4}{ }^{-}$: (at $20{ }^{\circ} \mathrm{C}$, cosolvent: 0.4 vol- $\% \mathrm{CH}_{3} \mathrm{CN}, \mathrm{J} \& \mathrm{M}$, detection at $630 \mathrm{~nm}$ )

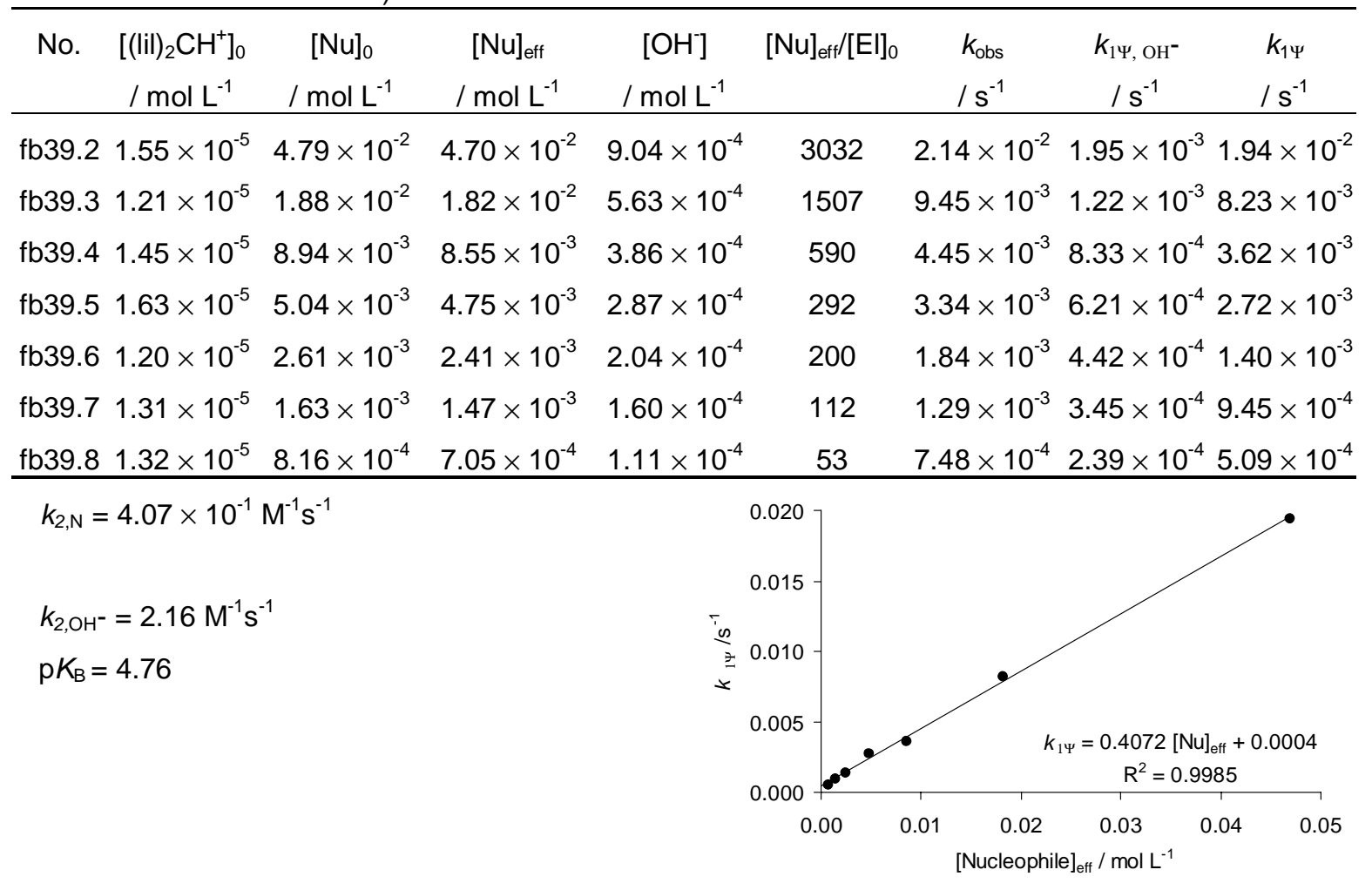


3.2. Reactivity parameters in water: $N=9.48 ; s=0.59$

\begin{tabular}{ccc}
\hline Reference electrophile & $E$ parameter & $k_{2, \mathrm{~N}}\left(20^{\circ} \mathrm{C}\right) / \mathrm{M}^{-1} \mathrm{~s}^{-1}$ \\
\hline (mor $)_{2} \mathrm{CH}^{+}$ & -5.53 & $2.51 \times 10^{2}$ \\
$(\mathrm{dma})_{2} \mathrm{CH}^{+}$ & -7.02 & 18.5 \\
$(\mathrm{pyr})_{2} \mathrm{CH}^{+}$ & -7.69 & 14.3 \\
$(\text { thq })_{2} \mathrm{CH}^{+}$ & -8.22 & 5.39 \\
$(\text { ind })_{2} \mathrm{CH}^{+}$ & -8.76 & 2.39 \\
$(\mathrm{jul})_{2} \mathrm{CH}^{+}$ & -9.45 & 1.41 \\
$(\text { lil })_{2} \mathrm{CH}^{+}$ & -10.04 & 0.407 \\
\hline
\end{tabular}

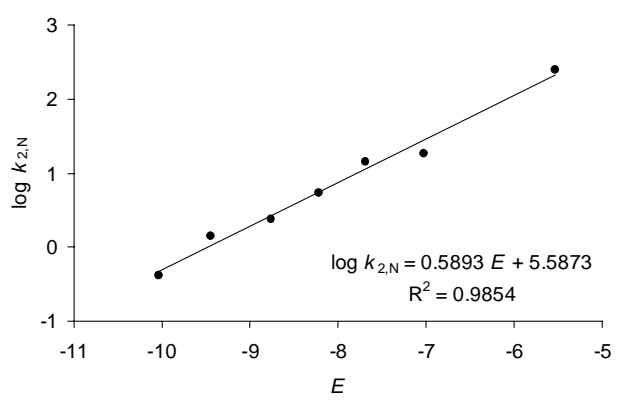

\section{Methylamine (1b)}

4.1. Rate constants in water

4.1.1. Reaction of methylamine (1b) with $(\mathrm{dma})_{2} \mathrm{CH}^{+} \mathrm{BF}_{4}{ }_{4}$ : (at $20{ }^{\circ} \mathrm{C}$, cosolvent: 1.5 vol- $\% \mathrm{CH}_{3} \mathrm{CN}$, stopped-flow, detection at $605 \mathrm{~nm}$ )

\begin{tabular}{|c|c|c|c|c|c|c|c|c|}
\hline No. & $\begin{array}{c}{\left[(\mathrm{dma})_{2} \mathrm{CH}^{+}\right]_{0}} \\
/ \mathrm{mol} \mathrm{L}^{-1}\end{array}$ & $\begin{array}{c}{[\mathrm{Nu}]_{0}} \\
/ \mathrm{mol} \mathrm{L}^{-1}\end{array}$ & $\begin{array}{c}{[\mathrm{Nu}]_{\mathrm{eff}}} \\
/ \mathrm{mol} \mathrm{L}^{-1}\end{array}$ & $\begin{array}{c}{\left[\mathrm{OH}^{-}\right]} \\
/ \mathrm{mol} \mathrm{L}^{-1}\end{array}$ & {$[\mathrm{Nu}]_{\mathrm{eff}} /[\mathrm{El}]_{0}$} & $\begin{array}{l}k_{\mathrm{obs}} \\
/ \mathrm{s}^{-1}\end{array}$ & $\begin{array}{c}k_{1 \Psi, \mathrm{OH}^{-}} \\
/ \mathrm{s}^{-1} \\
\end{array}$ & $\begin{array}{l}k_{1 \Psi} \\
/ \mathrm{s}^{-1}\end{array}$ \\
\hline ссу33.1 & $1.26 \times 10^{-5}$ & $2.88 \times 10^{-3}$ & $1.82 \times 10^{-3}$ & $1.06 \times 10^{-3}$ & 144 & 7.89 & 0.139 & 7.75 \\
\hline cсу33.2 & $1.26 \times 10^{-5}$ & $2.30 \times 10^{-3}$ & $1.38 \times 10^{-3}$ & $9.22 \times 10^{-4}$ & 109 & 5.93 & 0.121 & 5.81 \\
\hline ссу33.3 & $1.26 \times 10^{-5}$ & $1.73 \times 10^{-3}$ & $9.60 \times 10^{-4}$ & $7.70 \times 10^{-4}$ & 76 & 4.21 & 0.101 & 4.11 \\
\hline cсу33.4 & $1.26 \times 10^{-5}$ & $1.15 \times 10^{-3}$ & $5.62 \times 10^{-4}$ & $5.88 \times 10^{-4}$ & 45 & 2.52 & $7.71 \times 10^{-2}$ & 2.44 \\
\hline ссу33.5 & $1.26 \times 10^{-5}$ & $5.76 \times 10^{-4}$ & $2.13 \times 10^{-4}$ & $3.63 \times 10^{-4}$ & 17 & 0.972 & $4.75 \times 10^{-2}$ & 0.924 \\
\hline ссу33.6 & $1.26 \times 10^{-5}$ & $2.88 \times 10^{-4}$ & $7.42 \times 10^{-5}$ & $2.14 \times 10^{-4}$ & 6 & 0.307 & $2.80 \times 10^{-2}$ & 0.279 \\
\hline
\end{tabular}

$k_{2, \mathrm{~N}}=4.25 \times 10^{3} \mathrm{M}^{-1} \mathrm{~s}^{-1}$

$k_{2, \mathrm{OH}^{-}}=131 \mathrm{M}^{-1} \mathrm{~s}^{-1}$

$\mathrm{p} K_{\mathrm{B}}=3.21$

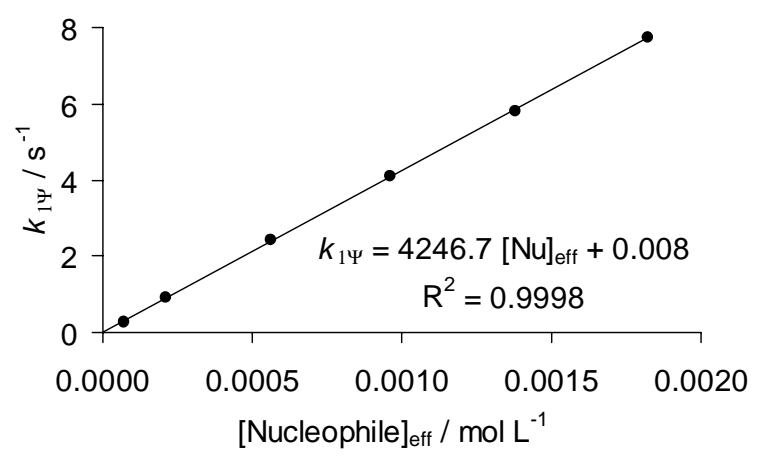


4.1.2. Reaction of methylamine (1b) with $\left(\right.$ pyr) ${ }_{2} \mathrm{CH}^{+} \mathrm{BF}_{4}{ }_{4}$ : (at $20{ }^{\circ} \mathrm{C}$, cosolvent: 0.2 vol- $\% \mathrm{CH}_{3} \mathrm{CN}$, stopped-flow, detection at $605 \mathrm{~nm}$ )

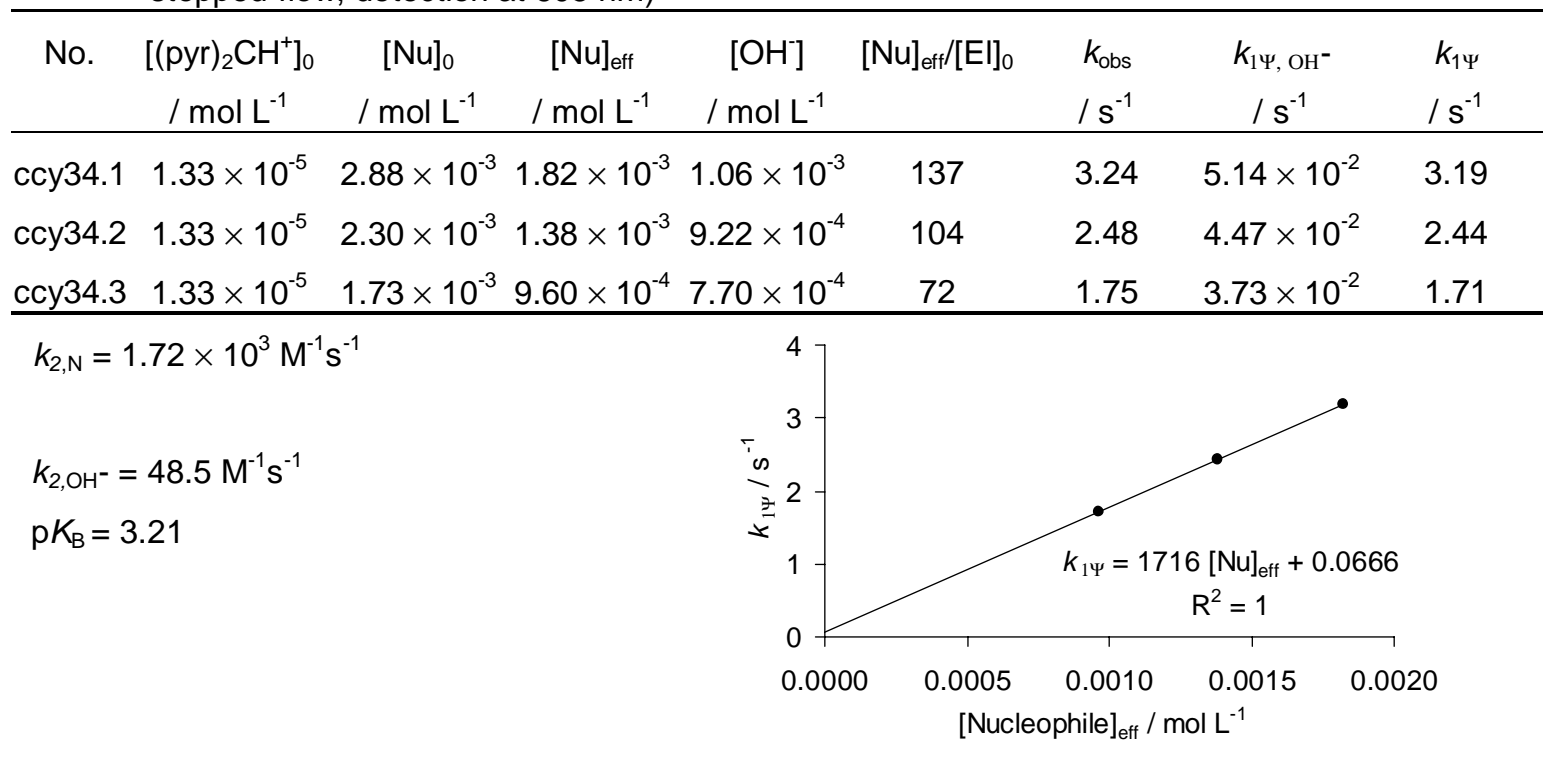

4.1.3. Reaction of methylamine (1b) with (thq) ${ }_{2} \mathrm{CH}^{+} \mathrm{BF}_{4}$ : (at $20^{\circ} \mathrm{C}$, cosolvent: 0.2 vol- $\% \mathrm{CH}_{3} \mathrm{CN}$, stopped-flow, detection at $615 \mathrm{~nm}$ )

\begin{tabular}{ccccccccc}
\hline No. & $\begin{array}{c}{\left[(\text { thq })_{2} \mathrm{CH}^{+}\right]_{0}} \\
/ \mathrm{mol} \mathrm{L}^{-1}\end{array}$ & $\begin{array}{c}{[\mathrm{Nu}]_{0}} \\
/ \mathrm{mol} \mathrm{L}^{-1}\end{array}$ & $\begin{array}{c}{[\mathrm{Nu}]_{\mathrm{eff}}} \\
/ \mathrm{mol} \mathrm{L}^{-1}\end{array}$ & $\begin{array}{c}{[\mathrm{OH}]} \\
/ \mathrm{mol} \mathrm{L}^{-1}\end{array}$ & $\begin{array}{c}\mathrm{Nu}_{\mathrm{eff}} /[\mathrm{El}]_{0} \\
\mathrm{k}_{\mathrm{obs}}\end{array}$ & $\begin{array}{c}k_{1 \Psi, \mathrm{OH}^{-}} \\
/ \mathrm{s}^{-1}\end{array}$ & $\begin{array}{c}k_{1 \Psi} \\
/ \mathrm{s}^{-1}\end{array}$ \\
\hline ccy35.1 & $1.63 \times 10^{-5}$ & $2.88 \times 10^{-3}$ & $1.82 \times 10^{-3}$ & $1.06 \times 10^{-3}$ & 112 & 1.71 & $2.50 \times 10^{-2}$ & 1.68 \\
ccy35.2 & $1.63 \times 10^{-5}$ & $2.30 \times 10^{-3}$ & $1.38 \times 10^{-3}$ & $9.22 \times 10^{-4}$ & 85 & 1.29 & $2.18 \times 10^{-2}$ & 1.27 \\
ccy35.3 & $1.63 \times 10^{-5}$ & $1.73 \times 10^{-3}$ & $9.60 \times 10^{-4}$ & $7.70 \times 10^{-4}$ & 59 & 0.916 & $1.82 \times 10^{-2}$ & 0.898 \\
ccy35.4 & $1.63 \times 10^{-5}$ & $1.15 \times 10^{-3}$ & $5.62 \times 10^{-4}$ & $5.88 \times 10^{-4}$ & 34 & 0.538 & $1.39 \times 10^{-2}$ & 0.524 \\
ccy35.5 & $1.63 \times 10^{-5}$ & $5.76 \times 10^{-4}$ & $2.13 \times 10^{-4}$ & $3.63 \times 10^{-4}$ & 13 & 0.214 & $8.56 \times 10^{-3}$ & 0.205 \\
ccy35.6 & $1.63 \times 10^{-5}$ & $2.88 \times 10^{-4}$ & $7.42 \times 10^{-5}$ & $2.14 \times 10^{-4}$ & 5 & $7.56 \times 10^{-2}$ & $5.05 \times 10^{-3}$ & $7.06 \times 10^{-2}$ \\
\hline
\end{tabular}

$k_{2, \mathrm{~N}}=9.21 \times 10^{2} \mathrm{M}^{-1} \mathrm{~s}^{-1}$

$k_{2, \mathrm{OH}}=23.6 \mathrm{M}^{-1} \mathrm{~s}^{-1}$

$\mathrm{p} K_{\mathrm{B}}=3.21$

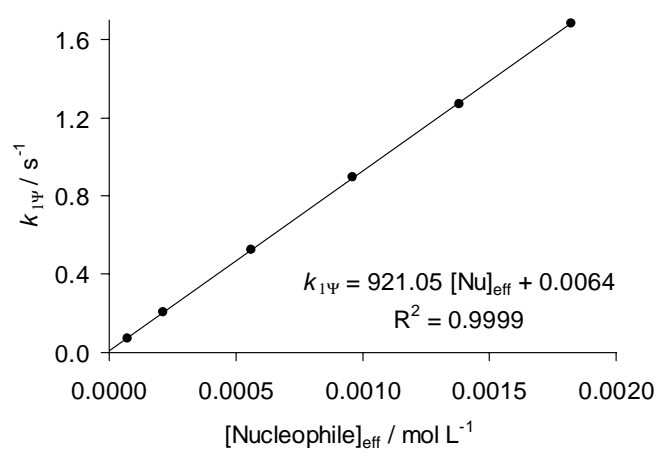


4.1.4. Reaction of methylamine (1b) with (ind) ${ }_{2} \mathrm{CH}^{+} \mathrm{BF}_{4}{ }_{4}$ : (at $20{ }^{\circ} \mathrm{C}$, cosolvent: 0.2 vol- $\% \mathrm{CH}_{3} \mathrm{CN}$, stopped-flow, detection at $614 \mathrm{~nm}$ )

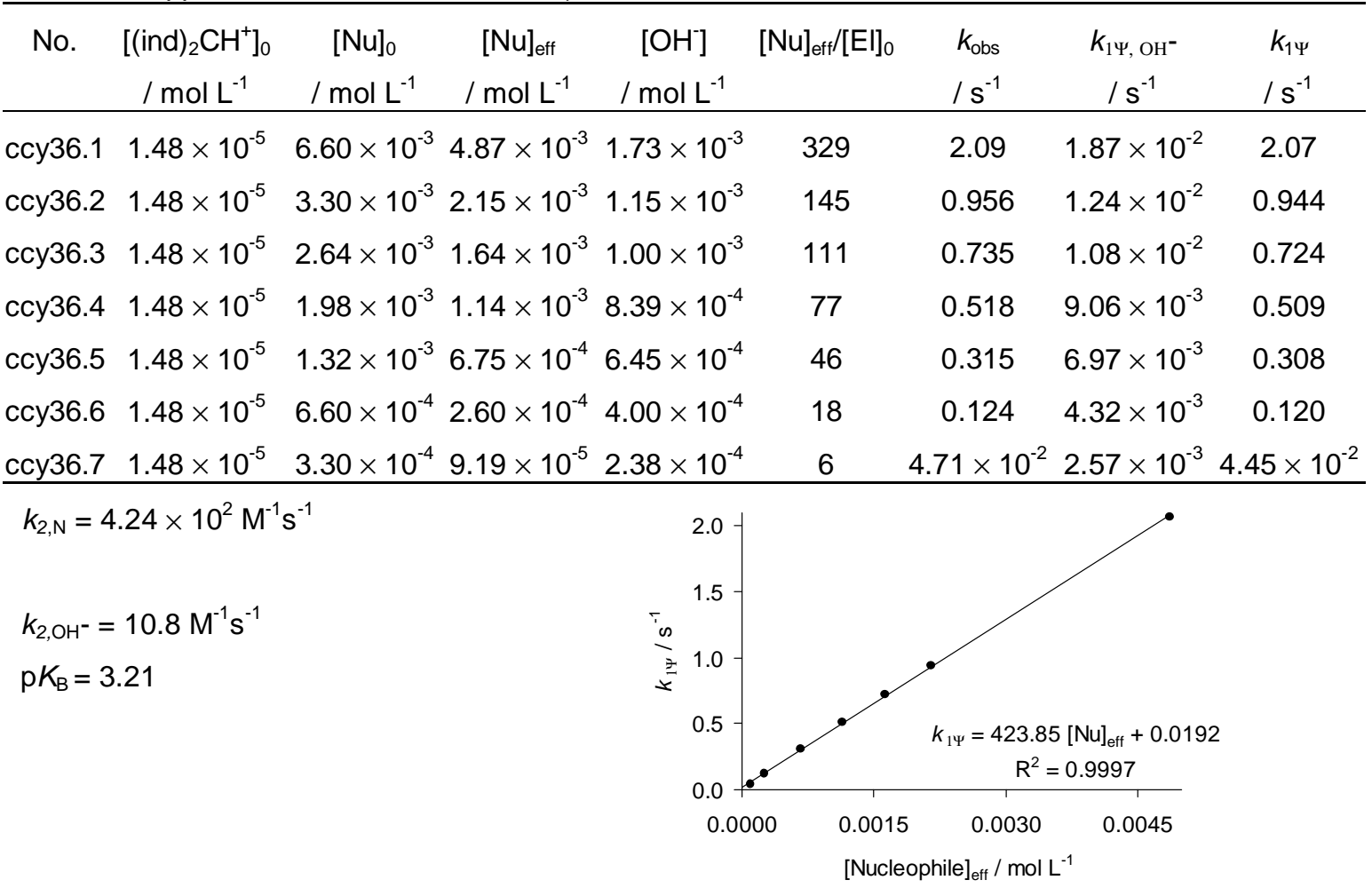

4.1.5. Reaction of methylamine (1b) with $(\mathrm{jul})_{2} \mathrm{CH}^{+} \mathrm{BF}_{4}{ }^{-}$: (at $20^{\circ} \mathrm{C}$, cosolvent: 0.2 vol- $\% \mathrm{CH}_{3} \mathrm{CN}$, stopped-flow, detection at $614 \mathrm{~nm}$ )

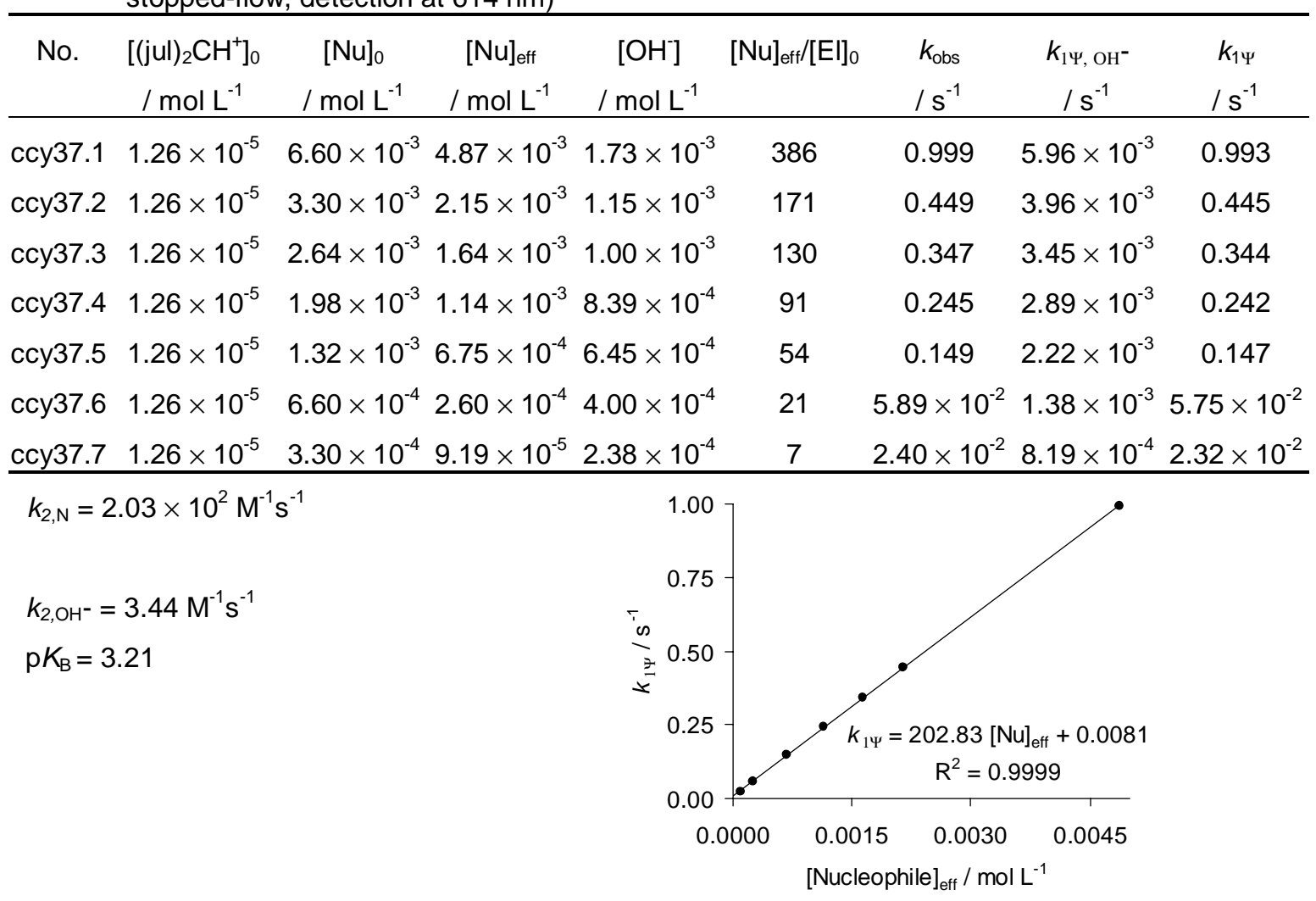


4.1.6. Reaction of methylamine (1b) with (lil) ${ }_{2} \mathrm{CH}^{+} \mathrm{BF}_{4}{ }^{-}$: (at $20{ }^{\circ} \mathrm{C}$, cosolvent: 0.2 vol- $\% \mathrm{CH}_{3} \mathrm{CN}$, stopped-flow, detection at $614 \mathrm{~nm}$ )

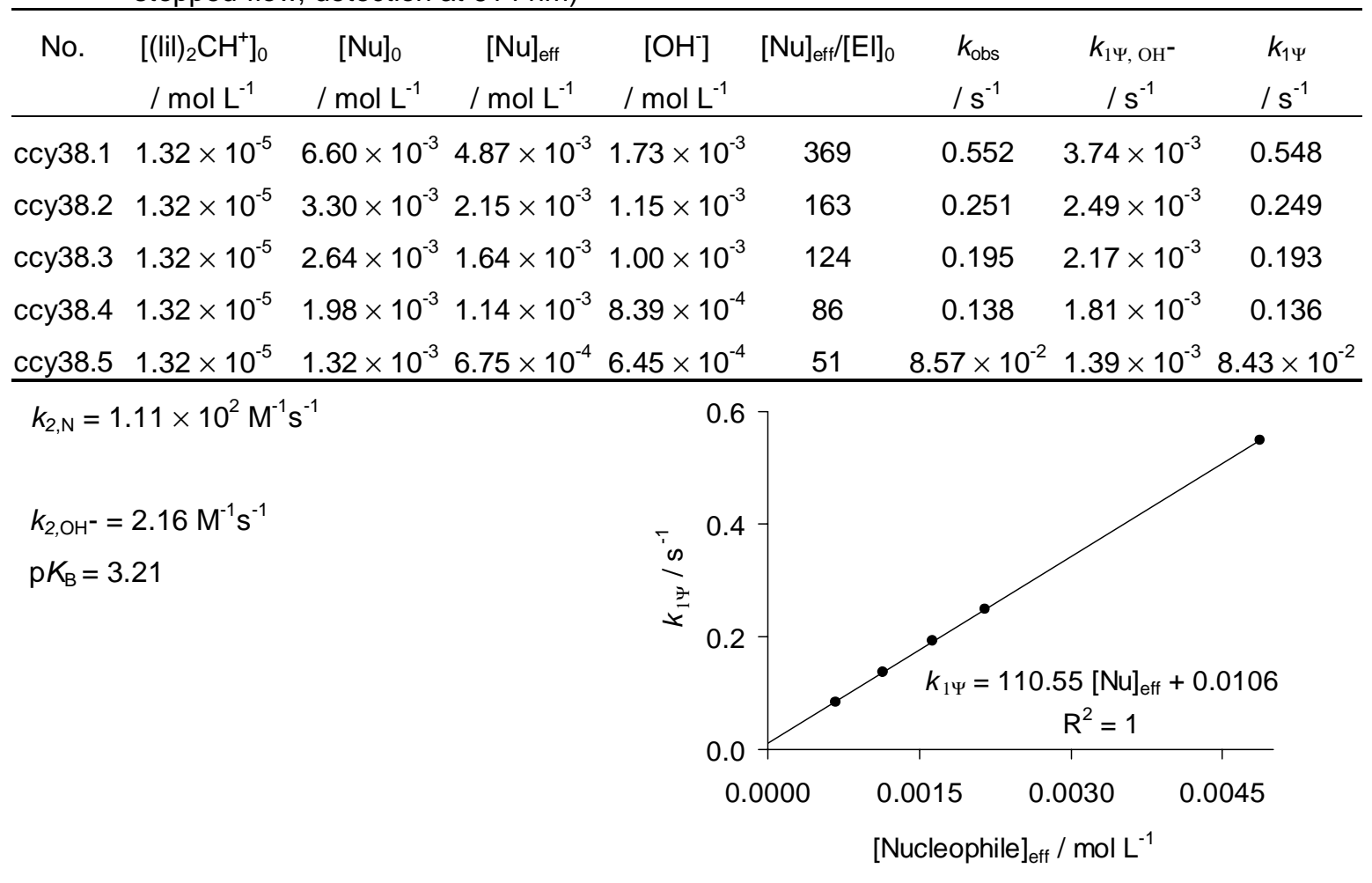

4.2. Reactivity parameters in water: $N=13.85 ; s=0.53$

\begin{tabular}{ccc}
\hline Reference electrophile & $E$ parameter & $k_{2, \mathrm{~N}}\left(20^{\circ} \mathrm{C}\right) / \mathrm{M}^{-1} \mathrm{~s}^{-1}$ \\
\hline$(\mathrm{dma})_{2} \mathrm{CH}^{+}$ & -7.02 & $4.25 \times 10^{3}$ \\
$(\text { pyr })_{2} \mathrm{CH}^{+}$ & -7.69 & $1.72 \times 10^{3}$ \\
$(\text { thq })_{2} \mathrm{CH}^{+}$ & -8.22 & $9.21 \times 10^{2}$ \\
$(\text { ind })_{2} \mathrm{CH}^{+}$ & -8.76 & $4.24 \times 10^{2}$ \\
$(\mathrm{jul})_{2} \mathrm{CH}^{+}$ & -9.45 & $2.03 \times 10^{2}$ \\
$(\mathrm{lil})_{2} \mathrm{CH}^{+}$ & -10.04 & $1.11 \times 10^{2}$ \\
\hline
\end{tabular}

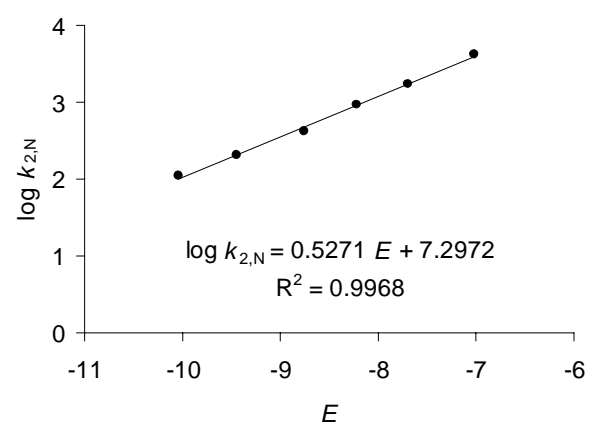




\section{Ethylamine (1c)}

5.1. Rate constants in water

5.1.1. Reaction of ethylamine (1c) with (dma) $)_{2} \mathrm{CH}^{+} \mathrm{BF}_{4}$ : (at $20^{\circ} \mathrm{C}$, cosolvent: 2.0 vol- $\% \mathrm{CH}_{3} \mathrm{CN}$, stopped-flow, detection at $615 \mathrm{~nm}$ )

\begin{tabular}{|c|c|c|c|c|c|c|c|c|}
\hline No. & $\begin{array}{c}{\left[(\mathrm{dma})_{2} \mathrm{CH}^{+}\right]_{0}} \\
/ \mathrm{mol} \mathrm{L}^{-1}\end{array}$ & $\begin{array}{c}{[\mathrm{Nu}]_{0}} \\
/ \mathrm{mol} \mathrm{L}^{-1}\end{array}$ & $\begin{array}{c}{[\mathrm{Nu}]_{\mathrm{eff}}} \\
/ \mathrm{mol} \mathrm{L}^{-1}\end{array}$ & $\begin{array}{c}{\left[\mathrm{OH}^{-}\right]} \\
/ \mathrm{mol} \mathrm{L}^{-1}\end{array}$ & {$[\mathrm{Nu}]_{\mathrm{eff}} /[\mathrm{El}]_{0}$} & $\begin{array}{l}k_{\mathrm{obs}} \\
/ \mathrm{s}^{-1}\end{array}$ & $\begin{array}{c}k_{1 \Psi, \mathrm{OH}^{-}} \\
/ \mathrm{s}^{-1}\end{array}$ & $\begin{array}{l}k_{1 \Psi} \\
/ s^{-1}\end{array}$ \\
\hline jo39.1 & $6.70 \times 10^{-5}$ & $1.05 \times 10^{-2}$ & $8.44 \times 10^{-3}$ & $2.06 \times 10^{-3}$ & 126 & 20.7 & 0.269 & 20.4 \\
\hline jo39.2 & $6.70 \times 10^{-5}$ & $5.25 \times 10^{-3}$ & $3.86 \times 10^{-3}$ & $1.39 \times 10^{-3}$ & 58 & 9.95 & 0.182 & 9.77 \\
\hline jo39.3 & $6.70 \times 10^{-5}$ & $3.68 \times 10^{-3}$ & $2.55 \times 10^{-3}$ & $1.13 \times 10^{-3}$ & 38 & 6.73 & 0.148 & 6.58 \\
\hline jo39.4 & $6.70 \times 10^{-5}$ & $2.10 \times 10^{-3}$ & $1.29 \times 10^{-3}$ & $8.05 \times 10^{-4}$ & 19 & 3.55 & 0.106 & 3.44 \\
\hline jo39.5 & $6.70 \times 10^{-5}$ & $1.05 \times 10^{-3}$ & $5.33 \times 10^{-4}$ & $5.17 \times 10^{-4}$ & 8 & 1.58 & $6.77 \times 10^{-2}$ & 1.51 \\
\hline
\end{tabular}

$$
\begin{aligned}
& k_{2, \mathrm{~N}}=2.39 \times 10^{3} \mathrm{M}^{-1} \mathrm{~s}^{-1} \\
& k_{2, \mathrm{OH}^{-}}=131 \mathrm{M}^{-1} \mathrm{~s}^{-1} \\
& \mathrm{p} K_{\mathrm{B}}=3.30
\end{aligned}
$$

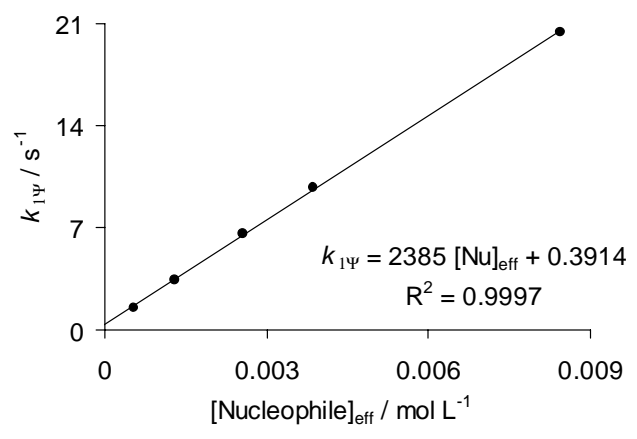

5.1.2. Reaction of ethylamine (1c) with $(\mathrm{pyr})_{2} \mathrm{CH}^{+} \mathrm{BF}_{4}$ : (at $20{ }^{\circ} \mathrm{C}$, cosolvent: 0.5 vol- $\% \mathrm{CH}_{3} \mathrm{CN}$, stopped-flow, detection at $605 \mathrm{~nm}$ )

\begin{tabular}{ccccccccc}
\hline No. & $\begin{array}{c}{\left[(\mathrm{pyr})_{2} \mathrm{CH}^{+}\right]_{0}} \\
/ \mathrm{mol} \mathrm{L}^{-1}\end{array}$ & $\begin{array}{c}{[\mathrm{Nu}]_{0}} \\
/ \mathrm{mol} \mathrm{L}^{-1}\end{array}$ & $\begin{array}{c}{[\mathrm{Nu}]_{\mathrm{eff}}} \\
/ \mathrm{mol} \mathrm{L}^{-1}\end{array}$ & $\begin{array}{c}{[\mathrm{OH}]} \\
/ \mathrm{mol} \mathrm{L}^{-1}\end{array}$ & {$[\mathrm{Nu}]_{\mathrm{eff}} /[\mathrm{El}]_{0}$} & $\begin{array}{c}k_{\mathrm{obs}} \\
/ \mathrm{s}^{-1}\end{array}$ & $\begin{array}{c}k_{1 \Psi, \mathrm{OH}^{-}} \\
/ \mathrm{s}^{-1}\end{array}$ & $\begin{array}{c}k_{1 \Psi} \\
/ \mathrm{s}^{-1}\end{array}$ \\
\hline j040.1 & $7.65 \times 10^{-6}$ & $1.05 \times 10^{-2}$ & $8.44 \times 10^{-3}$ & $2.06 \times 10^{-3}$ & 1104 & 8.37 & $9.98 \times 10^{-2}$ & 8.27 \\
j040.2 & $7.65 \times 10^{-6}$ & $5.25 \times 10^{-3}$ & $3.86 \times 10^{-3}$ & $1.39 \times 10^{-3}$ & 504 & 3.88 & $6.75 \times 10^{-2}$ & 3.81 \\
j040.3 & $7.65 \times 10^{-6}$ & $3.68 \times 10^{-3}$ & $2.55 \times 10^{-3}$ & $1.13 \times 10^{-3}$ & 333 & 2.81 & $5.48 \times 10^{-2}$ & 2.76 \\
j040.4 & $7.65 \times 10^{-6}$ & $2.10 \times 10^{-3}$ & $1.29 \times 10^{-3}$ & $8.05 \times 10^{-4}$ & 169 & 1.34 & $3.91 \times 10^{-2}$ & 1.30 \\
j040.5 & $7.65 \times 10^{-6}$ & $1.05 \times 10^{-3}$ & $5.33 \times 10^{-4}$ & $5.17 \times 10^{-4}$ & 70 & 0.593 & $2.51 \times 10^{-2}$ & 0.568 \\
\hline
\end{tabular}

$$
\begin{aligned}
& k_{2, \mathrm{~N}}=9.70 \times 10^{2} \mathrm{M}^{-1} \mathrm{~s}^{-1} \\
& k_{2, \mathrm{OH}^{-}}=48.5 \mathrm{M}^{-1} \mathrm{~s}^{-1} \\
& \mathrm{p} K_{\mathrm{B}}=3.30
\end{aligned}
$$

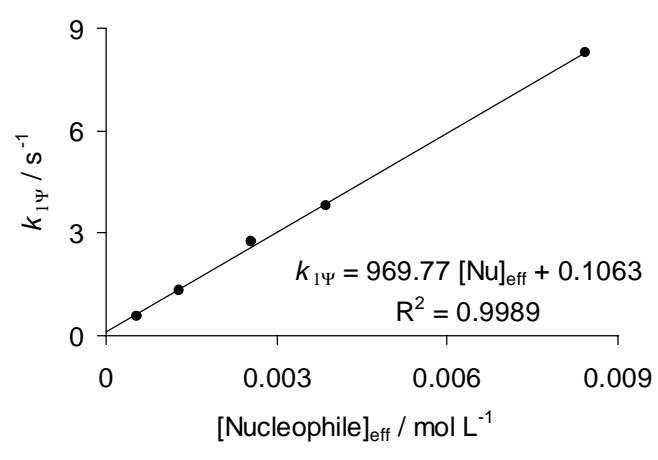


5.1.3. Reaction of ethylamine (1c) with (ind) ${ }_{2} \mathrm{CH}^{+} \mathrm{BF}_{4}{ }_{4}$ : (at $20{ }^{\circ} \mathrm{C}$, cosolvent: 0.2 vol- $\% \mathrm{CH}_{3} \mathrm{CN}$, stopped-flow, detection at $614 \mathrm{~nm}$ )

\begin{tabular}{|c|c|c|c|c|c|c|c|c|}
\hline No. & $\begin{array}{c}{\left[(\text { ind })_{2} \mathrm{CH}^{+}\right]_{0}} \\
/ \mathrm{mol} \mathrm{L}^{-1}\end{array}$ & $\begin{array}{c}{[\mathrm{Nu}]_{0}} \\
/ \mathrm{mol} \mathrm{L}^{-1}\end{array}$ & $\begin{array}{c}{[\mathrm{Nu}]_{\mathrm{eff}}} \\
/ \mathrm{mol} \mathrm{L}^{-1}\end{array}$ & $\begin{array}{c}{\left[\mathrm{OH}^{-}\right]} \\
/ \mathrm{mol} \mathrm{L}^{-1}\end{array}$ & {$[\mathrm{Nu}]_{\mathrm{eff}} /[\mathrm{El}]_{0}$} & $\begin{array}{l}k_{\mathrm{obs}} \\
/ \mathrm{s}^{-1}\end{array}$ & $\begin{array}{c}k_{1 \Psi, \mathrm{OH}^{-}} \\
/ \mathrm{s}^{-1}\end{array}$ & $\begin{array}{l}k_{1 \Psi} \\
/ s^{-1}\end{array}$ \\
\hline jo41.1 & $1.48 \times 10^{-5}$ & $1.05 \times 10^{-2}$ & $8.44 \times 10^{-3}$ & $2.06 \times 10^{-3}$ & 570 & 2.03 & $2.22 \times 10^{-2}$ & 2.01 \\
\hline jo41.2 & $1.48 \times 10^{-5}$ & $5.25 \times 10^{-3}$ & $3.86 \times 10^{-3}$ & $1.39 \times 10^{-3}$ & 261 & 0.965 & $1.50 \times 10^{-2}$ & 0.950 \\
\hline jo41.3 & $1.48 \times 10^{-5}$ & $3.68 \times 10^{-3}$ & $2.55 \times 10^{-3}$ & $1.13 \times 10^{-3}$ & 172 & 0.645 & $1.22 \times 10^{-2}$ & 0.633 \\
\hline jo41.4 & $1.48 \times 10^{-5}$ & $2.10 \times 10^{-3}$ & $1.29 \times 10^{-3}$ & $8.05 \times 10^{-4}$ & 87 & 0.334 & $8.70 \times 10^{-3}$ & 0.325 \\
\hline jo41.5 & $1.48 \times 10^{-5}$ & $1.05 \times 10^{-3}$ & $5.33 \times 10^{-4}$ & $5.17 \times 10^{-4}$ & 36 & 0.145 & $5.58 \times 10^{-3}$ & 0.139 \\
\hline
\end{tabular}

$$
\begin{aligned}
& k_{2, \mathrm{~N}}=2.36 \times 10^{2} \mathrm{M}^{-1} \mathrm{~s}^{-1} \\
& k_{2, \mathrm{OH}}=10.8 \mathrm{M}^{-1} \mathrm{~s}^{-1} \\
& \mathrm{p} K_{\mathrm{B}}=3.30
\end{aligned}
$$

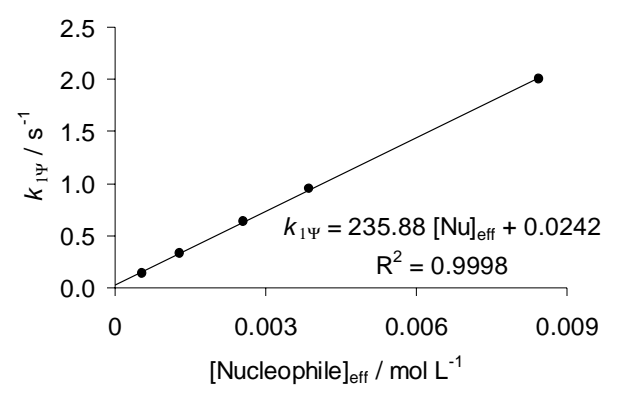

5.2. Reactivity parameters in water: $N=12.87 ; s=0.58$

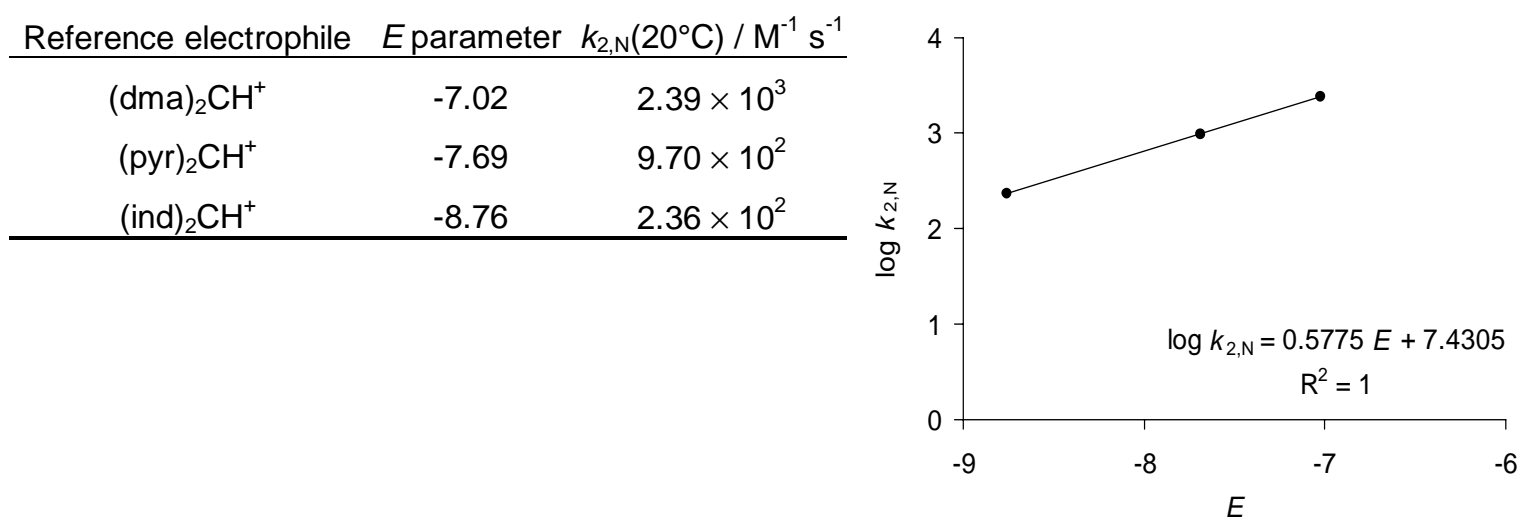




\section{Isopropylamine (1d)}

6.1. Rate constants in water

6.1.1. Reaction of Isopropylamine (1d) with $(\mathrm{dma})_{2} \mathrm{CH}^{+} \mathrm{BF}_{4}$ : (at $20^{\circ} \mathrm{C}$, cosolvent: 0.5 vol- $\% \mathrm{CH}_{3} \mathrm{CN}$, stopped-flow, detection at $610 \mathrm{~nm}$ )

\begin{tabular}{ccccccccc}
\hline No. & $\begin{array}{c}{\left[(\mathrm{dma})_{2} \mathrm{CH}^{+}\right]_{0}} \\
/ \mathrm{mol} \mathrm{L}^{-1}\end{array}$ & $\begin{array}{c}{[\mathrm{Nu}]_{0}} \\
/ \mathrm{mol} \mathrm{L}^{-1}\end{array}$ & $\begin{array}{c}{[\mathrm{Nu}]_{\mathrm{eff}}} \\
/ \mathrm{mol} \mathrm{L}^{-1}\end{array}$ & $\begin{array}{c}{[\mathrm{OH}]} \\
/ \mathrm{mol} \mathrm{L}^{-1}\end{array}$ & $\begin{array}{c}{[\mathrm{Nu}]_{\mathrm{eff}} /[\mathrm{El}]_{0}} \\
k_{\text {obs }}\end{array}$ & $\begin{array}{c}k_{1 \Psi, \mathrm{OH}^{-}} \\
/ \mathrm{s}^{-1}\end{array}$ & $\begin{array}{c}k_{1 \Psi} \\
/ \mathrm{s}^{-1}\end{array}$ & $/ \mathrm{s}^{-1}$ \\
\hline ccy196.1 & $5.95 \times 10^{-5}$ & $1.45 \times 10^{-2}$ & $1.22 \times 10^{-2}$ & $2.28 \times 10^{-3}$ & 205 & 8.45 & 0.299 & 8.15 \\
ccy196.2 & $5.95 \times 10^{-5}$ & $7.25 \times 10^{-3}$ & $5.69 \times 10^{-3}$ & $1.56 \times 10^{-3}$ & 96 & 4.03 & 0.204 & 3.83 \\
ccy196.3 & $5.95 \times 10^{-5}$ & $5.08 \times 10^{-3}$ & $3.81 \times 10^{-3}$ & $1.27 \times 10^{-3}$ & 64 & 2.69 & 0.167 & 2.52 \\
ccy196.5 & $5.95 \times 10^{-5}$ & $1.45 \times 10^{-3}$ & $8.48 \times 10^{-4}$ & $6.02 \times 10^{-4}$ & 14 & 0.617 & $7.88 \times 10^{-2}$ & 0.538 \\
\hline
\end{tabular}

$$
\begin{aligned}
& k_{2, \mathrm{~N}}=6.70 \times 10^{2} \mathrm{M}^{-1} \mathrm{~s}^{-1} \\
& k_{2, \mathrm{OH}^{-}}=131 \mathrm{M}^{-1} \mathrm{~s}^{-1} \\
& \mathrm{p} K_{\mathrm{B}}=3.37
\end{aligned}
$$

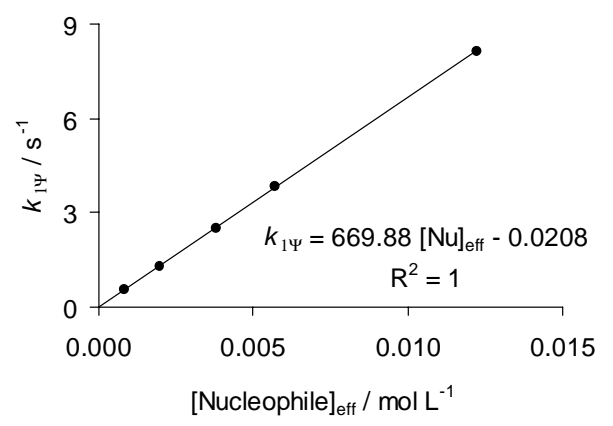

6.1.2. Reaction of Isopropylamine (1d) with $(\mathrm{pyr})_{2} \mathrm{CH}^{+} \mathrm{BF}_{4}$ : (at $20^{\circ} \mathrm{C}$, cosolvent: 0.1 vol-\% $\mathrm{CH}_{3} \mathrm{CN}$, stopped-flow, detection at $610 \mathrm{~nm}$ )

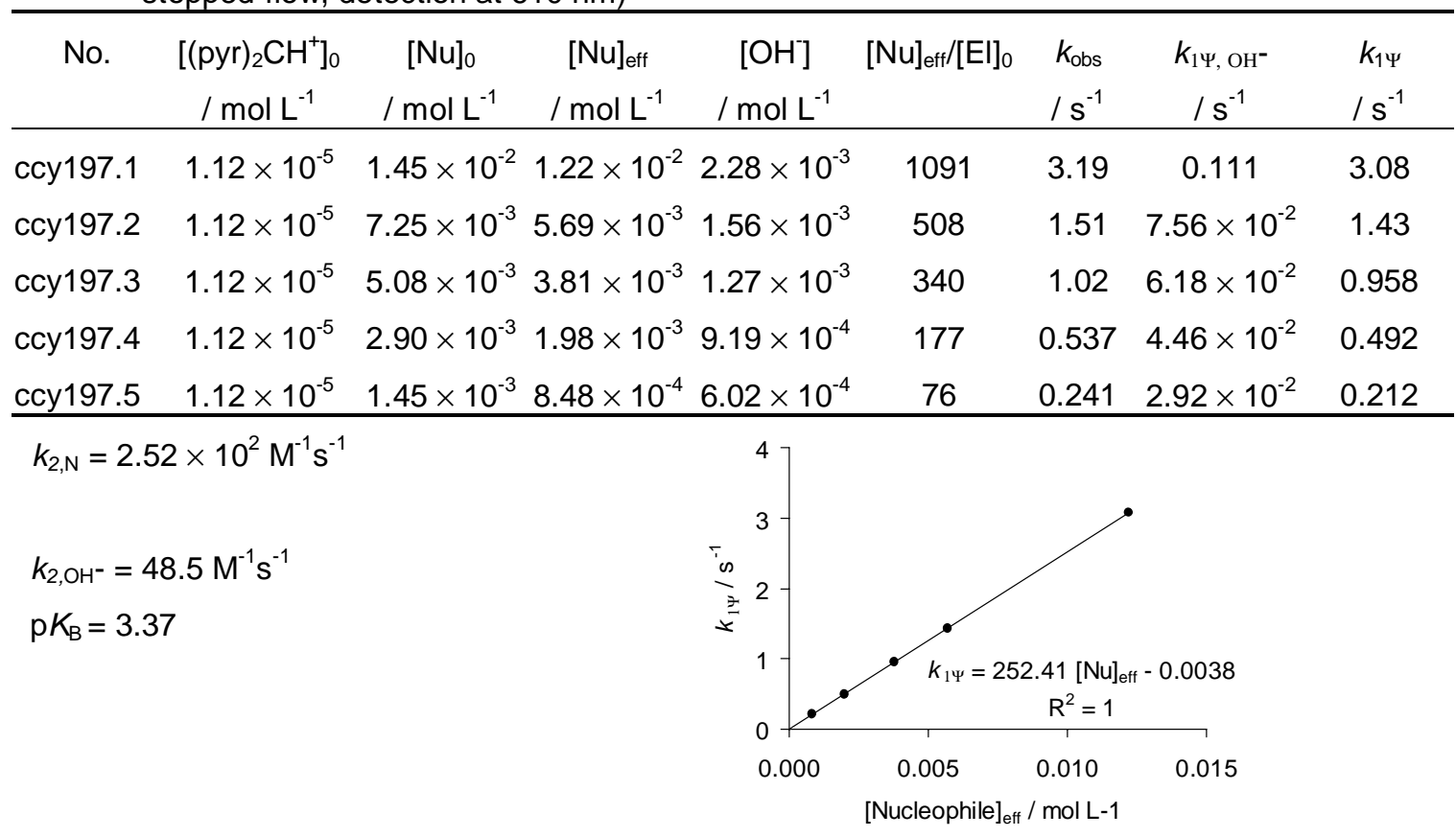


6.1.3. Reaction of Isopropylamine (1d) with (thq) $)_{2} \mathrm{CH}^{+} \mathrm{BF}_{4}^{-}$: (at $20{ }^{\circ} \mathrm{C}$, cosolvent: 0.2 vol- $\% \mathrm{CH}_{3} \mathrm{CN}$, stopped-flow, detection at $610 \mathrm{~nm}$ )

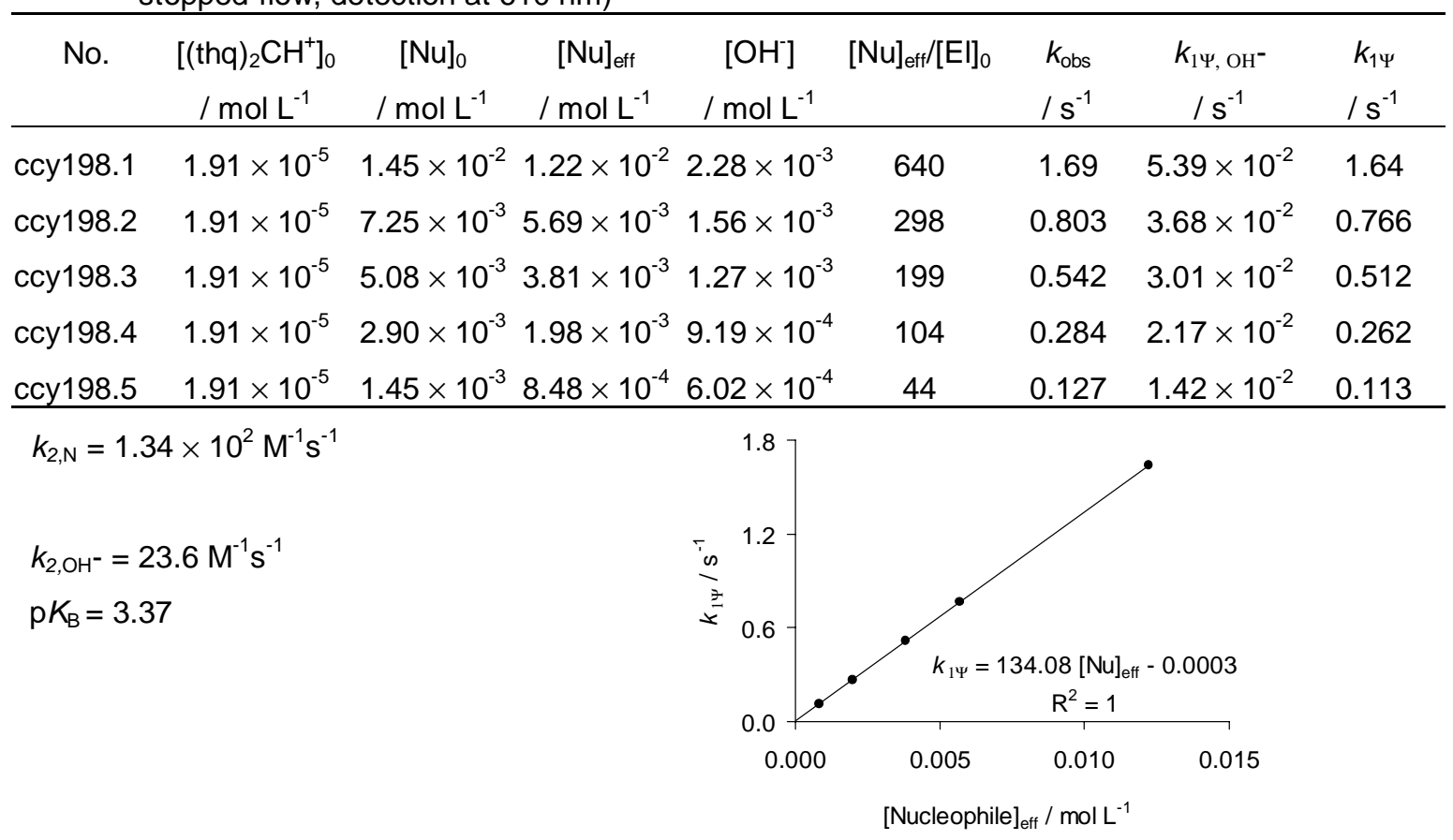

6.1.4. Reaction of Isopropylamine (1d) with $(\mathrm{jul})_{2} \mathrm{CH}^{+} \mathrm{BF}_{4}$ : (at $20{ }^{\circ} \mathrm{C}$, cosolvent: 0.2 vol- $\% \mathrm{CH}_{3} \mathrm{CN}$, stopped-flow, detection at $634 \mathrm{~nm}$ )

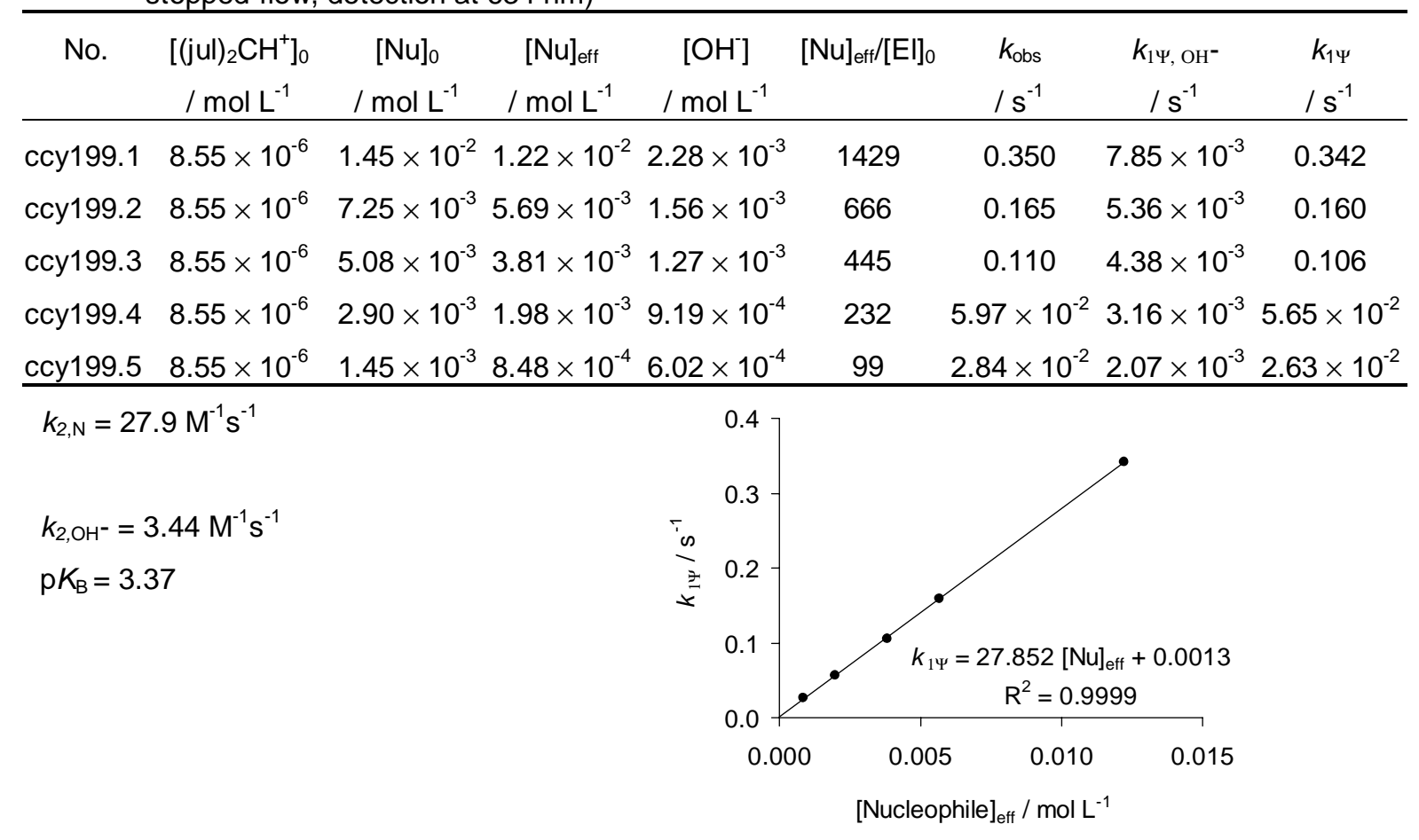


6.2. Reactivity parameters in water: $N=12.00 ; s=0.56$

\begin{tabular}{ccc}
\hline Reference electrophile & $E$ parameter & $k_{2, \mathrm{~N}}\left(20^{\circ} \mathrm{C}\right) / \mathrm{M}^{-1} \mathrm{~s}^{-1}$ \\
\hline$(\mathrm{dma})_{2} \mathrm{CH}^{+}$ & -7.02 & $6.70 \times 10^{2}$ \\
$(\mathrm{pyr})_{2} \mathrm{CH}^{+}$ & -7.69 & $2.52 \times 10^{2}$ \\
$(\text { thq })_{2} \mathrm{CH}^{+}$ & -8.22 & $1.34 \times 10^{2}$ \\
$(\mathrm{jul})_{2} \mathrm{CH}^{+}$ & -9.45 & 27.9 \\
\hline
\end{tabular}

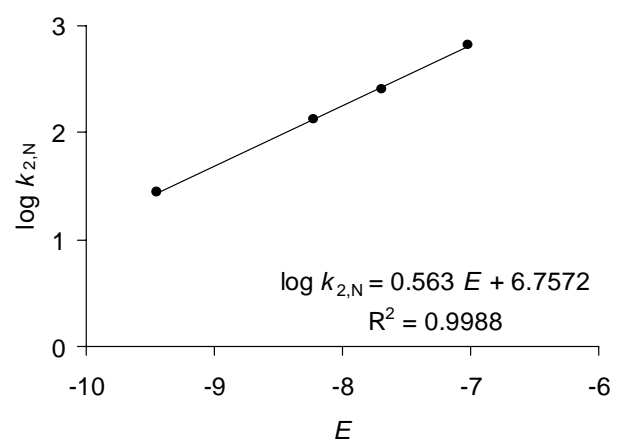

\section{7. tert-Butylamine (1e)}

7.1. Rate constants in water

7.1.1. Reaction of tert-Butylamine (1e) with (mor) ${ }_{2} \mathrm{CH}^{+} \mathrm{BF}_{4}$ : (at $20^{\circ} \mathrm{C}$, cosolvent: 9 vol- $\% \mathrm{CH}_{3} \mathrm{CN}$, stopped-flow, detection at $607 \mathrm{~nm}$ )

\begin{tabular}{ccccccccc}
\hline No. & $\begin{array}{c}{\left[(\mathrm{mor})_{2} \mathrm{CH}^{+}\right]_{0}} \\
/ \mathrm{mol} \mathrm{L}^{-1}\end{array}$ & $\begin{array}{c}{[\mathrm{Nu}]_{0}} \\
/ \mathrm{mol} \mathrm{L}^{-1}\end{array}$ & $\begin{array}{c}{[\mathrm{Nu}]_{\mathrm{eff}}} \\
/ \mathrm{mol} \mathrm{L}^{-1}\end{array}$ & $\begin{array}{c}{[\mathrm{OH}]} \\
/ \mathrm{mol} \mathrm{L}^{-1}\end{array}$ & {$[\mathrm{Nu}]_{\mathrm{eff}} /[\mathrm{El}]_{0}$} & $\begin{array}{c}k_{\mathrm{obs}} \\
/ \mathrm{s}^{-1}\end{array}$ & $\begin{array}{c}k_{1 \Psi, \mathrm{OH}^{-}} \\
/ \mathrm{s}^{-1}\end{array}$ & $\begin{array}{c}k_{1 \Psi} \\
/ \mathrm{s}^{-1}\end{array}$ \\
\hline fb201.1 & $1.72 \times 10^{-5}$ & $7.04 \times 10^{-3}$ & $5.12 \times 10^{-3}$ & $1.92 \times 10^{-3}$ & 297 & 14.5 & 2.04 & 12.5 \\
fb201.2 & $1.72 \times 10^{-5}$ & $4.22 \times 10^{-3}$ & $2.80 \times 10^{-3}$ & $1.42 \times 10^{-3}$ & 163 & 9.09 & 1.51 & 7.58 \\
fb201.3 & $1.72 \times 10^{-5}$ & $2.81 \times 10^{-3}$ & $1.70 \times 10^{-3}$ & $1.11 \times 10^{-3}$ & 99 & 6.97 & 1.18 & 5.79 \\
fb201.4 & $1.72 \times 10^{-5}$ & $1.41 \times 10^{-3}$ & $6.99 \times 10^{-4}$ & $7.11 \times 10^{-4}$ & 41 & 3.85 & 0.754 & 3.10 \\
fb201.5 & $1.72 \times 10^{-5}$ & $7.04 \times 10^{-4}$ & $2.65 \times 10^{-4}$ & $4.39 \times 10^{-4}$ & 15 & 2.34 & 0.465 & 1.88 \\
\hline
\end{tabular}

$$
\begin{aligned}
& k_{2, \mathrm{~N}}=2.14 \times 10^{3} \mathrm{M}^{-1} \mathrm{~s}^{-1} \\
& k_{2, \mathrm{OH}^{-}}=1060 \mathrm{M}^{-1} \mathrm{~s}^{-1} \\
& \mathrm{p} K_{\mathrm{B}}=3.14
\end{aligned}
$$

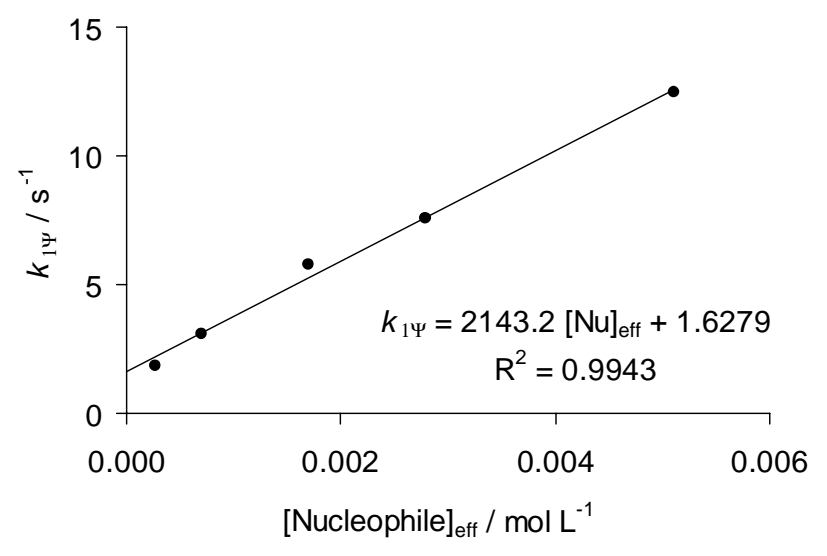


7.1.2. Reaction of tert-Butylamine (1e) with $(\mathrm{dma})_{2} \mathrm{CH}^{+} \mathrm{BF}_{4}{ }^{\circ}$ : (at $20^{\circ} \mathrm{C}$, cosolvent: 0.5 vol- $\% \mathrm{CH}_{3} \mathrm{CN}$, stopped-flow, detection at $610 \mathrm{~nm}$ )

\begin{tabular}{|c|c|c|c|c|c|c|c|c|}
\hline No. & $\begin{array}{c}{\left[(\mathrm{dma})_{2} \mathrm{CH}^{+}\right]_{0}} \\
/ \mathrm{mol} \mathrm{L}^{-1}\end{array}$ & $\begin{array}{c}{[\mathrm{Nu}]_{0}} \\
/ \mathrm{mol} \mathrm{L}^{-1}\end{array}$ & $\begin{array}{c}{[\mathrm{Nu}]_{\mathrm{eff}}} \\
/ \mathrm{mol} \mathrm{L}^{-1}\end{array}$ & $\begin{array}{c}{\left[\mathrm{OH}^{-}\right]} \\
/ \mathrm{mol} \mathrm{L}^{-1}\end{array}$ & {$[\mathrm{Nu}]_{\mathrm{eff}} /[\mathrm{El}]_{0}$} & $\begin{array}{l}k_{\mathrm{obs}} \\
/ \mathrm{s}^{-1}\end{array}$ & $\begin{array}{c}k_{1 \Psi, \mathrm{OH}^{-}} \\
/ \mathrm{s}^{-1}\end{array}$ & $\begin{array}{l}k_{1 \Psi} \\
/ s^{-1}\end{array}$ \\
\hline ccy111.1 & $1.08 \times 10^{-4}$ & $6.48 \times 10^{-3}$ & $4.65 \times 10^{-3}$ & $1.83 \times 10^{-3}$ & 43 & 1.02 & 0.240 & 0.780 \\
\hline ccy 111.2 & $1.08 \times 10^{-4}$ & $3.24 \times 10^{-3}$ & $2.03 \times 10^{-3}$ & $1.21 \times 10^{-3}$ & 19 & 0.534 & 0.159 & 0.375 \\
\hline ccy111.3 & $1.08 \times 10^{-4}$ & $2.27 \times 10^{-3}$ & $1.30 \times 10^{-3}$ & $9.70 \times 10^{-4}$ & 12 & 0.373 & 0.127 & 0.246 \\
\hline ccy 111.4 & $1.08 \times 10^{-4}$ & $1.30 \times 10^{-3}$ & $6.26 \times 10^{-4}$ & $6.74 \times 10^{-4}$ & 6 & 0.220 & $8.82 \times 10^{-2}$ & 0.132 \\
\hline
\end{tabular}

$$
\begin{aligned}
& k_{2, \mathrm{~N}}=1.60 \times 10^{2} \mathrm{M}^{-1} \mathrm{~s}^{-1} \\
& k_{2, \mathrm{OH}^{-}}=131 \mathrm{M}^{-1} \mathrm{~s}^{-1} \\
& \mathrm{p} K_{\mathrm{B}}=3.14
\end{aligned}
$$

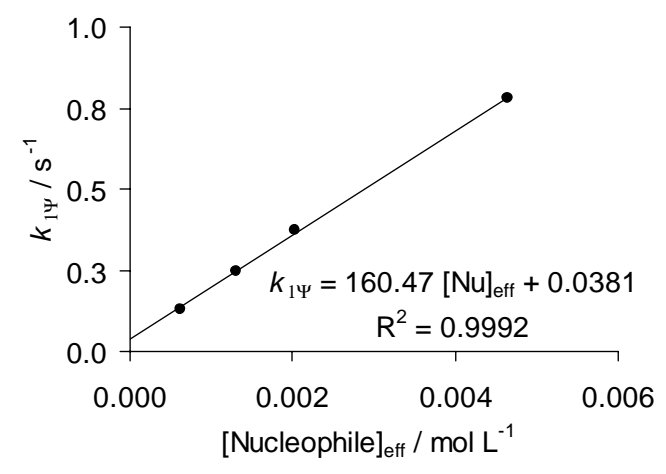

7.1.3. Reaction of tert-Butylamine (1e) with (pyr) ${ }_{2} \mathrm{CH}^{+} \mathrm{BF}_{4}{ }_{4}$ : (at $20^{\circ} \mathrm{C}$, cosolvent: 0.2 vol- $\% \mathrm{CH}_{3} \mathrm{CN}$, stopped-flow, detection at $610 \mathrm{~nm}$ )

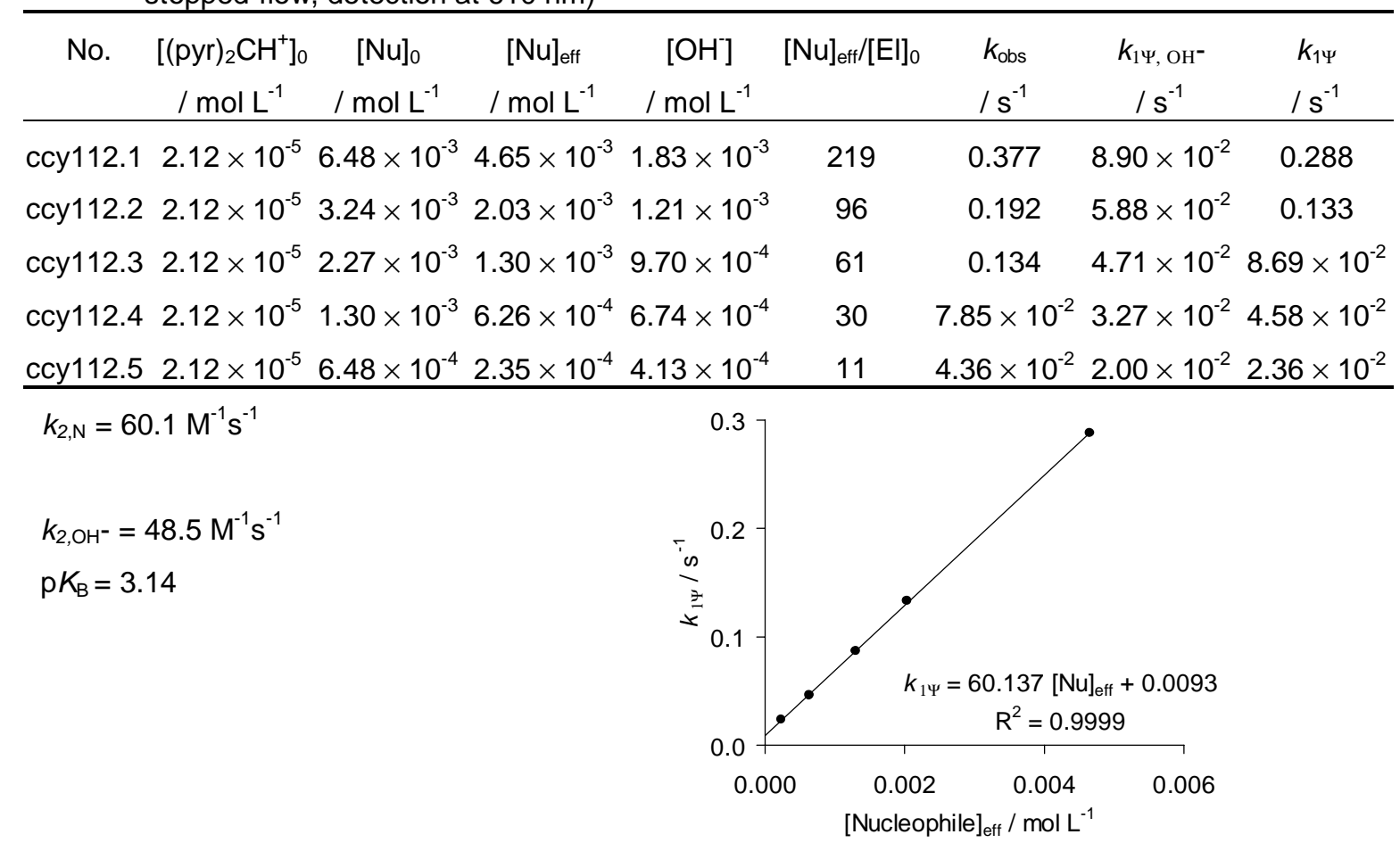


7.1.4. Reaction of tert-Butylamine (1e) with (thq) ${ }_{2} \mathrm{CH}^{+} \mathrm{BF}_{4}{ }_{4}$ : (at $20^{\circ} \mathrm{C}$, cosolvent: 0.2 vol- $\% \mathrm{CH}_{3} \mathrm{CN}$, stopped-flow, detection at $610 \mathrm{~nm}$ )

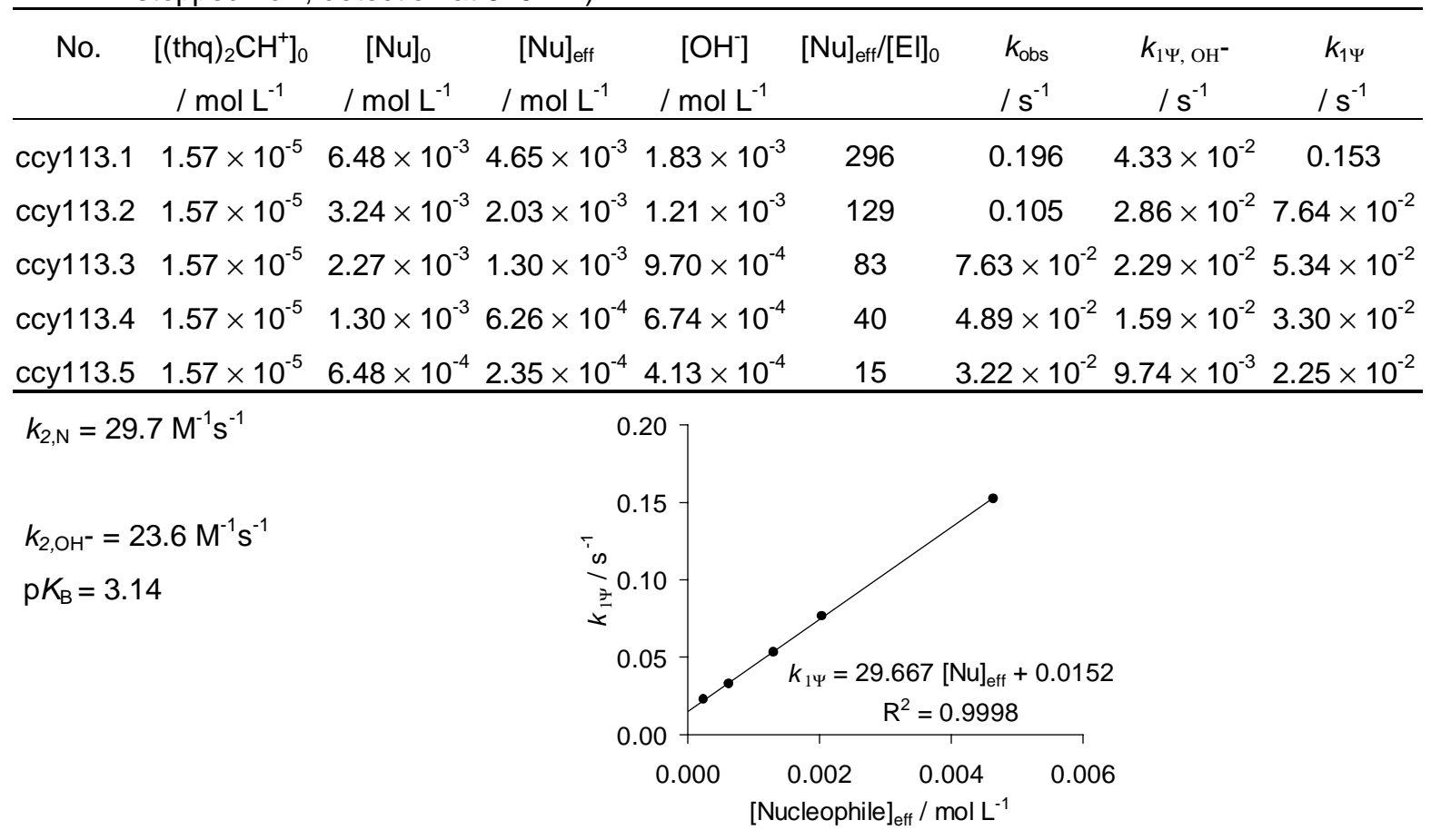

7.1.5. Reaction of tert-Butylamine (1e) with (ind) ${ }_{2} \mathrm{CH}^{+} \mathrm{BF}_{4}{ }_{4}$ : (at $20{ }^{\circ} \mathrm{C}$, cosolvent: 0.2 vol- $\% \mathrm{CH}_{3} \mathrm{CN}$, J\&M, detection at $614 \mathrm{~nm}$ )

\begin{tabular}{|c|c|c|c|c|c|c|c|c|}
\hline No. & $\begin{array}{c}{\left[(\text { ind })_{2} \mathrm{CH}^{+}\right]_{0}} \\
/ \mathrm{mol} \mathrm{L}^{-1}\end{array}$ & $\begin{array}{c}{[\mathrm{Nu}]_{0}} \\
/ \mathrm{mol} \mathrm{L}^{-1}\end{array}$ & $\begin{array}{c}{[\mathrm{Nu}]_{\mathrm{eff}}} \\
/ \mathrm{mol} \mathrm{L}^{-1}\end{array}$ & $\begin{array}{c}{\left[\mathrm{OH}^{-}\right]} \\
/ \mathrm{mol} \mathrm{L}^{-1}\end{array}$ & {$[\mathrm{Nu}]_{\mathrm{eff}} /[\mathrm{El}]_{0}$} & $\begin{array}{l}k_{\mathrm{obs}} \\
/ \mathrm{s}^{-1}\end{array}$ & $\begin{array}{c}k_{1 \Psi, \mathrm{OH}^{-}} \\
/ \mathrm{s}^{-1}\end{array}$ & $\begin{array}{l}k_{1 \Psi} \\
/ \mathrm{s}^{-1}\end{array}$ \\
\hline fb200.1 & $2.20 \times 10^{-5}$ & $2.92 \times 10^{-3}$ & $1.78 \times 10^{-3}$ & $1.14 \times 10^{-3}$ & 81 & $3.31 \times 10^{-2}$ & $1.23 \times 10^{-2}$ & $2.08 \times 10^{-2}$ \\
\hline fb200.2 & $2.20 \times 10^{-5}$ & $2.32 \times 10^{-3}$ & $1.34 \times 10^{-3}$ & $9.84 \times 10^{-4}$ & 61 & $2.69 \times 10^{-2}$ & $1.06 \times 10^{-2}$ & $1.63 \times 10^{-2}$ \\
\hline fb200.3 & $2.24 \times 10^{-5}$ & $1.19 \times 10^{-3}$ & $5.56 \times 10^{-4}$ & $6.34 \times 10^{-4}$ & 25 & $1.55 \times 10^{-2}$ & $6.85 \times 10^{-3}$ & $8.65 \times 10^{-3}$ \\
\hline $\mathrm{fb} 200.4$ & $2.26 \times 10^{-5}$ & $5.98 \times 10^{-4}$ & $2.09 \times 10^{-4}$ & $3.89 \times 10^{-4}$ & 9 & $9.39 \times 10^{-3}$ & $4.20 \times 10^{-3}$ & $5.19 \times 10^{-3}$ \\
\hline \multicolumn{2}{|c|}{$k_{2, \mathrm{~N}}=9.90 \mathrm{M}^{-1} \mathrm{~s}^{-1}$} & & & $\begin{array}{l}0.025 \\
0.020\end{array}$ & & & & \\
\hline \multicolumn{2}{|c|}{$k_{2, \mathrm{OH}^{-}}=10.8 \mathrm{M}^{-1} \mathrm{~s}^{-1}$} & & & is 0.015 & & & & \\
\hline \multirow[t]{2}{*}{$\mathrm{p} K_{\mathrm{B}}=3$} & .14 & & & $\begin{array}{r} \pm 0.010 \\
0.005\end{array}$ & & $R^{2}=0$ & 31 & \\
\hline & & & & & $\begin{array}{r}.0000 \quad 0.0005 \\
\text { [Nucles }\end{array}$ & $\begin{array}{l}50.00100 .001 \\
\text { ophile }]_{\text {eff }} / \mathrm{mol}^{-}\end{array}$ & $15 \quad 0.0020$ & \\
\hline
\end{tabular}


7.1.6. Reaction of tert-Butylamine (1e) with (lil) ${ }_{2} \mathrm{CH}^{+} \mathrm{BF}_{4}{ }_{4}$ : (at $20{ }^{\circ} \mathrm{C}$, cosolvent: 0.2 vol-\% $\mathrm{CH}_{3} \mathrm{CN}$, $J \& M$, detection at $614 \mathrm{~nm}$ )

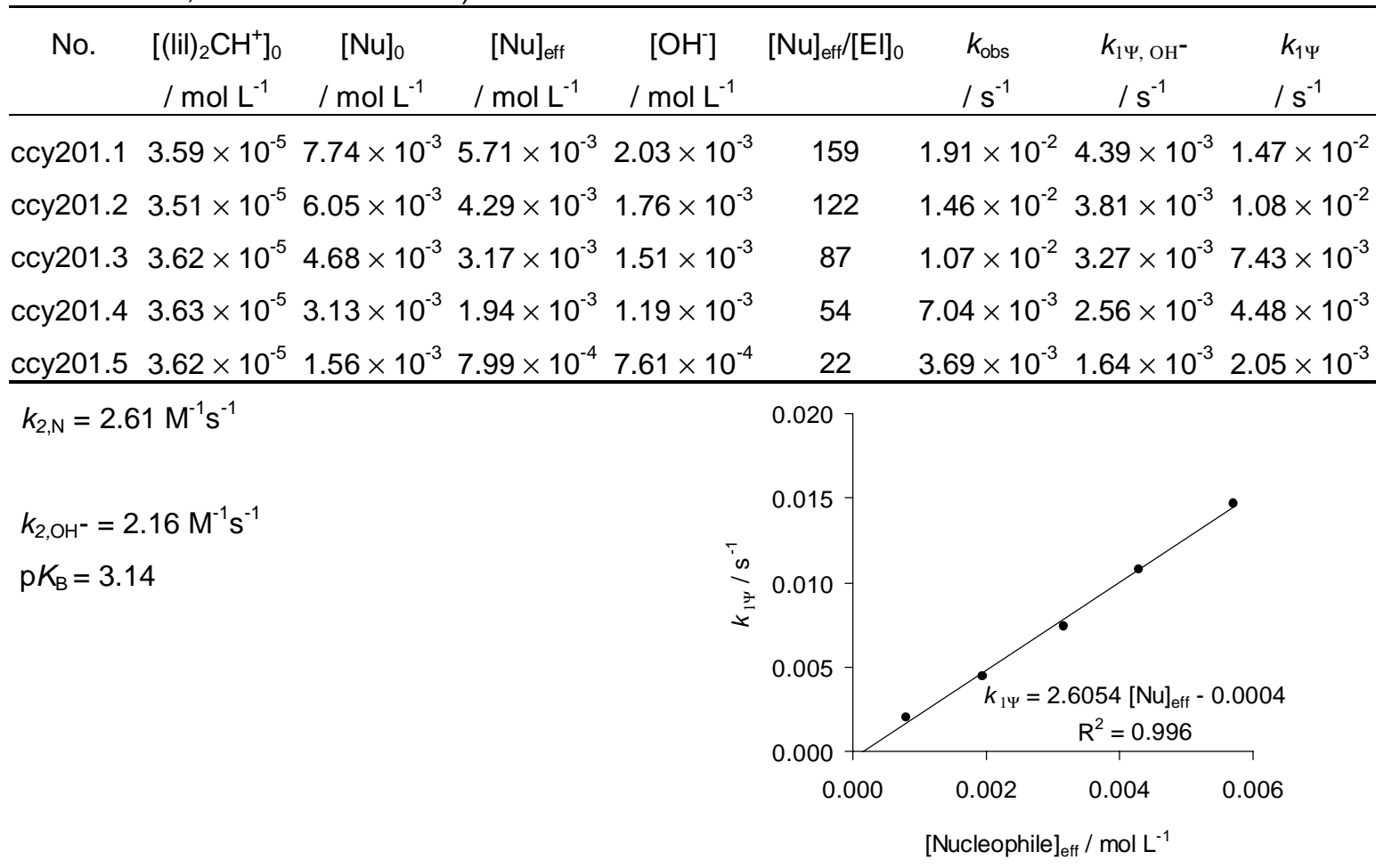

7.2. Reactivity parameters in water: $N=10.48 ; s=0.65$

\begin{tabular}{ccc}
\hline Reference electrophile & $E$ parameter & $k_{2, \mathrm{~N}}\left(20^{\circ} \mathrm{C}\right) / \mathrm{M}^{-1} \mathrm{~s}^{-1}$ \\
\hline (mor $)_{2} \mathrm{CH}^{+}$ & -5.53 & $2.14 \times 10^{3}$ \\
$(\mathrm{dma})_{2} \mathrm{CH}^{+}$ & -7.02 & $1.60 \times 10^{2}$ \\
$(\mathrm{pyr})_{2} \mathrm{CH}^{+}$ & -7.69 & 60.1 \\
$(\mathrm{thq})_{2} \mathrm{CH}^{+}$ & -8.22 & 29.7 \\
$(\text { ind })_{2} \mathrm{CH}^{+}$ & -8.76 & 9.90 \\
$(\mathrm{lil})_{2} \mathrm{CH}^{+}$ & -10.04 & 2.61 \\
\hline
\end{tabular}

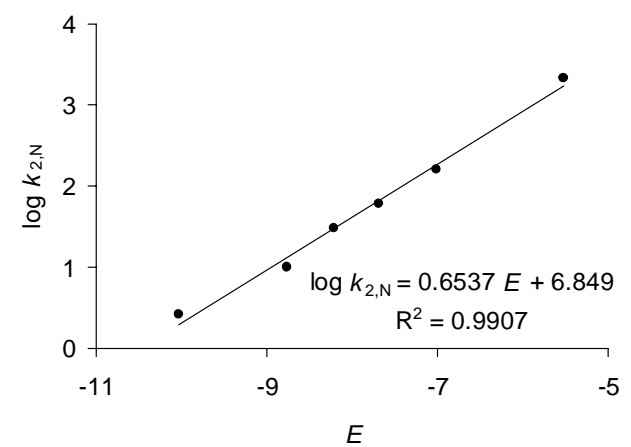




\section{Glycinenitrile (1f)}

8.1. Rate constants and equilibrium constants in water

8.1.1. Reaction of glycinenitrile (1f) with (dma) ${ }_{2} \mathrm{CH}^{+} \mathrm{BF}_{4}{ }^{-}$: (at $20{ }^{\circ} \mathrm{C}$, cosolvent: 1.5 vol- $\% \mathrm{CH}_{3} \mathrm{CN}$, stopped-flow, detection at $610 \mathrm{~nm}$, $\left.[\mathrm{Nu}]_{\text {eff }}=[\mathrm{Nu}]_{0}, k_{\mathrm{obs}}=k_{1 \Psi}\right)$

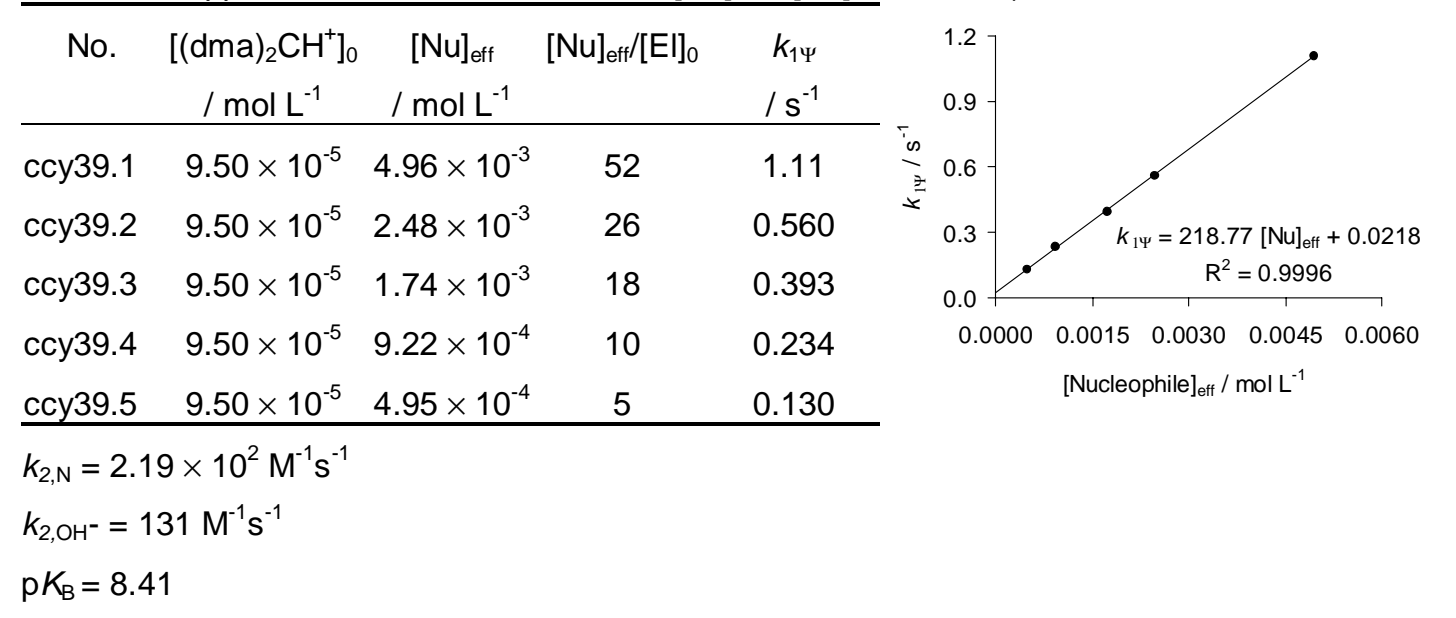

8.1.2. Reaction of glycinenitrile (1f) with $(\mathrm{pyr})_{2} \mathrm{CH}^{+} \mathrm{BF}_{4}^{-}$: (at $20^{\circ} \mathrm{C}$, cosolvent: 0.2 vol- $\% \mathrm{CH}_{3} \mathrm{CN}$, stopped-flow, detection at $610 \mathrm{~nm}$; $\left.[\mathrm{Nu}]_{\text {eff }}=[\mathrm{Nu}]_{0}, k_{\mathrm{obs}}=k_{1 \Psi}\right)$

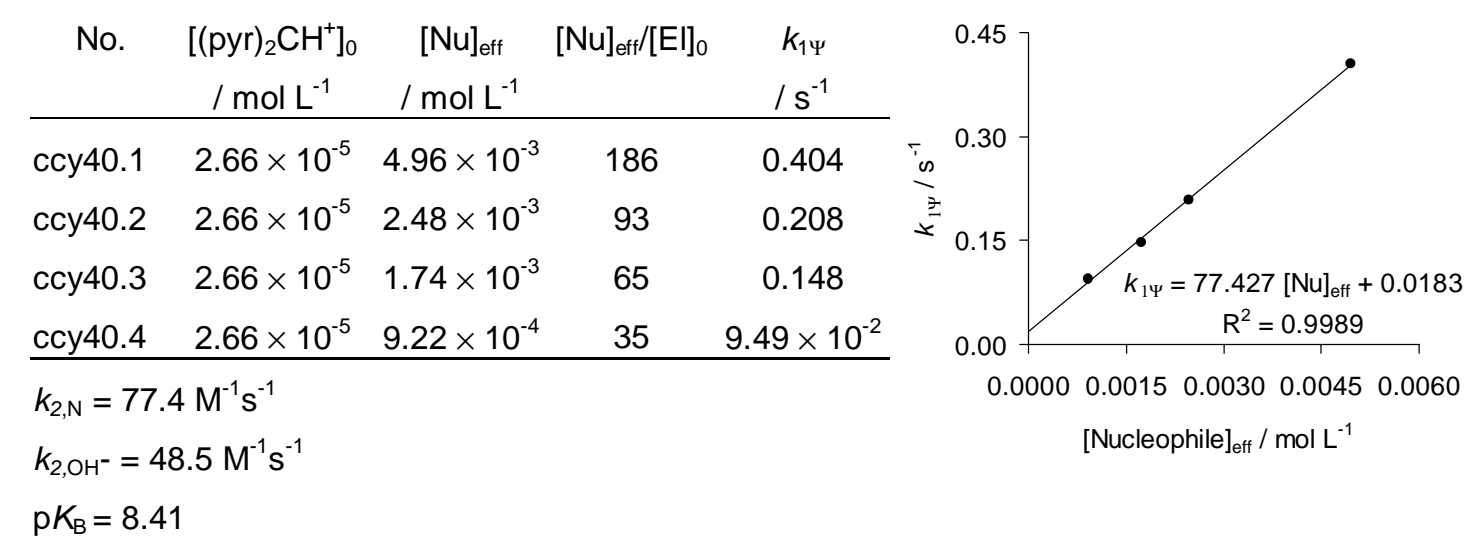


8.1.3. Reaction of glycinenitrile (1f) with (thq) ${ }_{2} \mathrm{CH}^{+} \mathrm{BF}_{4}{ }^{-}$: (at $20^{\circ} \mathrm{C}$, cosolvent: 0.2 vol- $\% \mathrm{CH}_{3} \mathrm{CN}$, stopped-flow, detection at $610 \mathrm{~nm}$, $\left.[\mathrm{Nu}]_{\text {eff }}=[\mathrm{Nu}]_{0}, k_{\mathrm{obs}}=k_{1 \Psi}\right)$

\begin{tabular}{|c|c|c|c|c|c|}
\hline No. & $\begin{array}{c}{\left[(\text { thq })_{2} \mathrm{CH}^{+}\right]_{0}} \\
/ \mathrm{mol} \mathrm{L}^{-1}\end{array}$ & $\begin{array}{c}{[\mathrm{Nu}]_{\mathrm{eff}}} \\
/ \mathrm{mol} \mathrm{L}^{-1}\end{array}$ & {$[\mathrm{Nu}]_{\mathrm{eff}} /[\mathrm{El}]_{0}$} & $\begin{array}{l}k_{1 \Psi} \\
/ \mathrm{s}^{-1} \\
\end{array}$ & $\left.\begin{array}{l}0.25 \\
0.20\end{array}\right]$ \\
\hline ccy41.1 & $1.63 \times 10^{-5}$ & $4.96 \times 10^{-3}$ & 304 & 0.219 & is 0.15 \\
\hline ccy41.2 & $1.63 \times 10^{-5}$ & $2.48 \times 10^{-3}$ & 152 & 0.116 & $\geq 0.10$ \\
\hline ccy41.3 & $1.63 \times 10^{-5}$ & $1.74 \times 10^{-3}$ & 107 & $8.64 \times 10^{-2}$ & $\begin{array}{l}9.129[\mathrm{Nu}]_{\mathrm{eff}}+0.022 \\
R^{2}=0.9974\end{array}$ \\
\hline ccy41.4 & $1.63 \times 10^{-5}$ & $9.22 \times 10^{-4}$ & 57 & $6.00 \times 10^{-2}$ & 0.00 \\
\hline ccy41.5 & $1.63 \times 10^{-5}$ & $4.95 \times 10^{-4}$ & 30 & $4.54 \times 10^{-2}$ & {$[\text { Nucleophile }]_{\text {eff }} / \mathrm{mol} \mathrm{L}^{-1}$} \\
\hline \multicolumn{6}{|c|}{$k_{2, \mathrm{~N}}=39.1 \mathrm{M}^{-1} \mathrm{~s}^{-1} ; k_{-1}=2.3 \times 10^{-2} \mathrm{~s}^{-1}$} \\
\hline \multicolumn{6}{|c|}{$K=k_{2, \mathrm{~N}} / k_{-1}=1.7 \times 10^{3} \mathrm{M}^{-1}$} \\
\hline \multicolumn{6}{|c|}{$k_{2, \mathrm{OH}^{-}}=23.6 \mathrm{M}^{-1} \mathrm{~s}^{-1}$} \\
\hline \multicolumn{6}{|c|}{$\mathrm{p} K_{\mathrm{B}}=8.41$} \\
\hline
\end{tabular}

8.1.4. Reaction of glycinenitrile (1f) with (ind) $\mathrm{CH}^{+} \mathrm{BF}_{4}{ }_{4}$ : (at $20^{\circ} \mathrm{C}$, cosolvent: 0.2 vol- $\% \mathrm{CH}_{3} \mathrm{CN}$, stopped-flow and J\&M, detection at $\left.614 \mathrm{~nm},[\mathrm{Nu}]_{\text {eff }}=[\mathrm{Nu}]_{0}, k_{\mathrm{obs}}=k_{1 \Psi}\right)$

\begin{tabular}{|c|c|c|c|c|}
\hline No. & $\begin{array}{c}{\left[(\text { ind })_{2} \mathrm{CH}^{+}\right]_{0}} \\
/ \mathrm{mol} \mathrm{L}^{-1}\end{array}$ & $\begin{array}{c}{[\mathrm{Nu}]_{\mathrm{eff}}} \\
/ \mathrm{mol} \mathrm{L}^{-1}\end{array}$ & {$[\mathrm{Nu}]_{\mathrm{eff}} /[\mathrm{El}]_{0}$} & $\begin{array}{l}k_{1 \Psi} \\
/ \mathrm{s}^{-1}\end{array}$ \\
\hline ccy55.1 ${ }^{[a]}$ & $1.48 \times 10^{-5}$ & $8.51 \times 10^{-3}$ & 575 & 0.158 \\
\hline $\operatorname{ccy} 55.2^{[a]}$ & $1.48 \times 10^{-5}$ & $5.68 \times 10^{-3}$ & 383 & 0.108 \\
\hline $\operatorname{ccy} 55.3^{[a]}$ & $1.48 \times 10^{-5}$ & $2.80 \times 10^{-3}$ & 189 & $5.67 \times 10^{-2}$ \\
\hline $\operatorname{ccy} 55.4^{[\mathrm{a}]}$ & $1.48 \times 10^{-5}$ & $2.27 \times 10^{-3}$ & 153 & $4.70 \times 10^{-2}$ \\
\hline $\operatorname{ccy} 55.5^{[a]}$ & $1.48 \times 10^{-5}$ & $1.70 \times 10^{-3}$ & 115 & $3.70 \times 10^{-2}$ \\
\hline $\operatorname{ccy} 55.6^{[a]}$ & $1.48 \times 10^{-5}$ & $1.14 \times 10^{-3}$ & 77 & $2.71 \times 10^{-2}$ \\
\hline $\operatorname{ccy} 55.8^{[b]}$ & $1.89 \times 10^{-5}$ & $6.48 \times 10^{-4}$ & 34 & $1.74 \times 10^{-2}$ \\
\hline $\operatorname{ccy} 55.9^{[b]}$ & $1.90 \times 10^{-5}$ & $5.23 \times 10^{-4}$ & 28 & $1.57 \times 10^{-2}$ \\
\hline $\operatorname{ccy} 55.7^{[b]}$ & $1.90 \times 10^{-5}$ & $4.78 \times 10^{-4}$ & 25 & $1.37 \times 10^{-2}$ \\
\hline
\end{tabular}

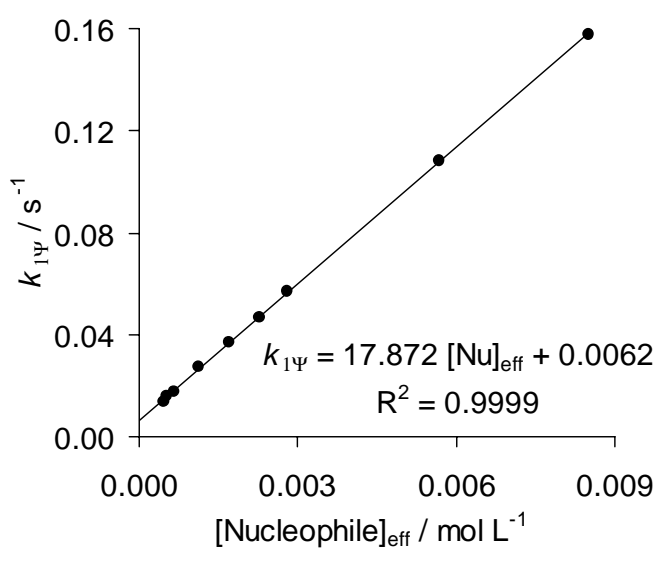

[a] Method: Stopped-flow

[b] Method: J\&M

$k_{2, \mathrm{~N}}=17.9 \mathrm{M}^{-1} \mathrm{~s}^{-1} ; k_{-1}=6.2 \times 10^{-3} \mathrm{~s}^{-1} ; K=k_{2, \mathrm{~N}} / k_{-1}=2.9 \times 10^{3} \mathrm{M}^{-1}$

$k_{2, \mathrm{OH}^{-}}=10.8 \mathrm{M}^{-1} \mathrm{~s}^{-1} ; \mathrm{p} K_{\mathrm{B}}=8.41$ 
8.1.5. Reaction of glycinenitrile (1f) with $(\mathrm{jul})_{2} \mathrm{CH}^{+} \mathrm{BF}_{4}{ }_{4}$ : (at $20^{\circ} \mathrm{C}$, cosolvent: 0.1 vol- $\% \mathrm{CH}_{3} \mathrm{CN}$, J\&M, detection at $\left.634 \mathrm{~nm},[\mathrm{Nu}]_{\mathrm{eff}}=[\mathrm{Nu}]_{0}, k_{\mathrm{obs}}=k_{1 \Psi}\right)$

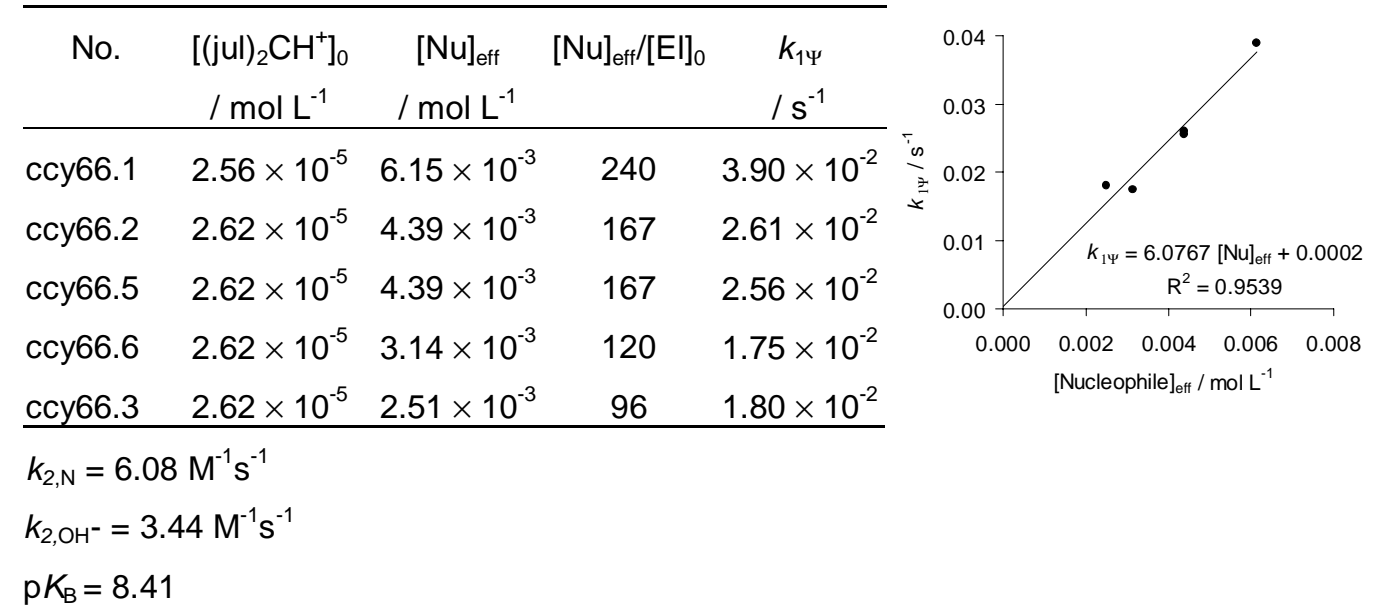

8.1.6. Reaction of glycinenitrile (1f) with (lil) $)_{2} \mathrm{CH}^{+} \mathrm{BF}_{4}^{-}$: (at $20^{\circ} \mathrm{C}$, cosolvent: 0.2 vol- $\% \mathrm{CH}_{3} \mathrm{CN}, \mathrm{J} \& \mathrm{M}$, detection at $630 \mathrm{~nm}$, $\left.[\mathrm{Nu}]_{\text {eff }}=[\mathrm{Nu}]_{0}, k_{\mathrm{obs}}=k_{1 \Psi}\right)$

\begin{tabular}{|c|c|c|c|c|c|}
\hline No. & $\begin{array}{c}{\left[(\mathrm{lil})_{2} \mathrm{CH}^{+}\right]_{0}} \\
/ \mathrm{mol} \mathrm{L}^{-1}\end{array}$ & \multicolumn{2}{|c|}{$\begin{array}{c}{[\mathrm{Nu}]_{\mathrm{eff}}} \\
/ \mathrm{mol} \mathrm{L}^{-1}\end{array}$} & {$[\mathrm{Nu}]_{\mathrm{eff}} /[\mathrm{El}]_{0}$} & $\begin{array}{l}k_{1 \Psi} \\
/ s^{-1}\end{array}$ \\
\hline ccy204.1 & $2.16 \times 10^{-5}$ & \multicolumn{2}{|c|}{$5.43 \times 10^{-3}$} & 251 & $1.95 \times 10^{-2}$ \\
\hline ccy204.2 & $2.17 \times 10^{-5}$ & \multicolumn{2}{|c|}{$4.37 \times 10^{-3}$} & 201 & $1.58 \times 10^{-2}$ \\
\hline ccy204.3 & $2.19 \times 10^{-5}$ & \multicolumn{2}{|c|}{$3.30 \times 10^{-3}$} & 151 & $1.42 \times 10^{-2}$ \\
\hline ccy204.4 & $2.14 \times 10^{-5}$ & \multicolumn{2}{|c|}{$2.16 \times 10^{-3}$} & 101 & $8.57 \times 10^{-3}$ \\
\hline ccy204.5 & $2.23 \times 10^{-5}$ & \multicolumn{2}{|c|}{$1.12 \times 10^{-3}$} & 50 & $5.80 \times 10^{-3}$ \\
\hline $\begin{array}{l}k_{2, \mathrm{~N}}=3.20 \\
k_{2, \mathrm{OH}^{-}}=2 . \\
\mathrm{p} K_{\mathrm{B}}=8.41\end{array}$ & $\begin{array}{l}\mathrm{M}^{-1} \mathrm{~s}^{-1} ; k_{-1}=2 \\
16 \mathrm{M}^{-1} \mathrm{~s}^{-1}\end{array}$ & $2.3 \times$ & $10^{-3} \mathrm{~s}^{-1}$ & $; K=k_{2, \mathrm{~N}} /$ & $k_{-1}=1.4 \times 10^{3} \mathrm{M}$ \\
\hline \multicolumn{6}{|c|}{ Reactivity parameters in water: $N=10.80 ; s=0.61$} \\
\hline Refere & ence electropl & & E parar & meter & ${ }_{2, N}\left(20^{\circ} \mathrm{C}\right) / \mathrm{M}^{-1} \mathrm{~s}^{-1}$ \\
\hline & $(\mathrm{dma})_{2} \mathrm{CH}^{+}$ & & -7.0 & 02 & $2.19 \times 10^{2}$ \\
\hline & $(\text { pyr })_{2} \mathrm{CH}^{+}$ & & -7.6 & 69 & 77.4 \\
\hline & $(\text { thq })_{2} \mathrm{CH}^{+}$ & & -8.2 & 22 & 39.1 \\
\hline & (ind $)_{2} \mathrm{CH}^{+}$ & & -8.7 & 76 & 17.9 \\
\hline & $(\mathrm{jul})_{2} \mathrm{CH}^{+}$ & & -9.4 & 45 & 6.08 \\
\hline & $(\mathrm{lil})_{2} \mathrm{CH}^{+}$ & & -10. & .04 & 3.20 \\
\hline
\end{tabular}

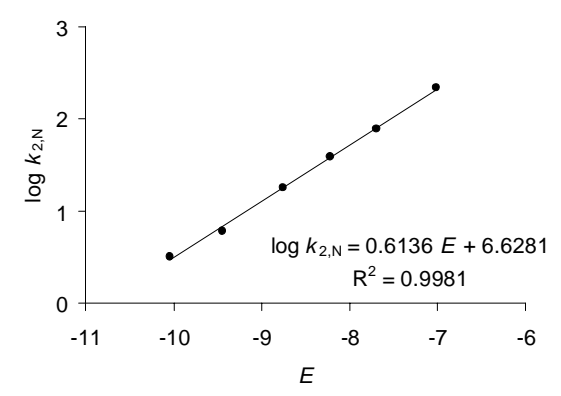




\section{Methyl glycinate $(1 \mathrm{~g})$}

9.1. Rate constants in water

9.1.1. Reaction of methyl glycinate $(\mathbf{1 g})$ with $(\mathrm{dma})_{2} \mathrm{CH}^{+} \mathrm{BF}_{4}{ }_{4}$ : (at $20^{\circ} \mathrm{C}$, cosolvent: 0.5 vol-\% $\mathrm{CH}_{3} \mathrm{CN}$, stopped-flow, detection at $610 \mathrm{~nm}$ )

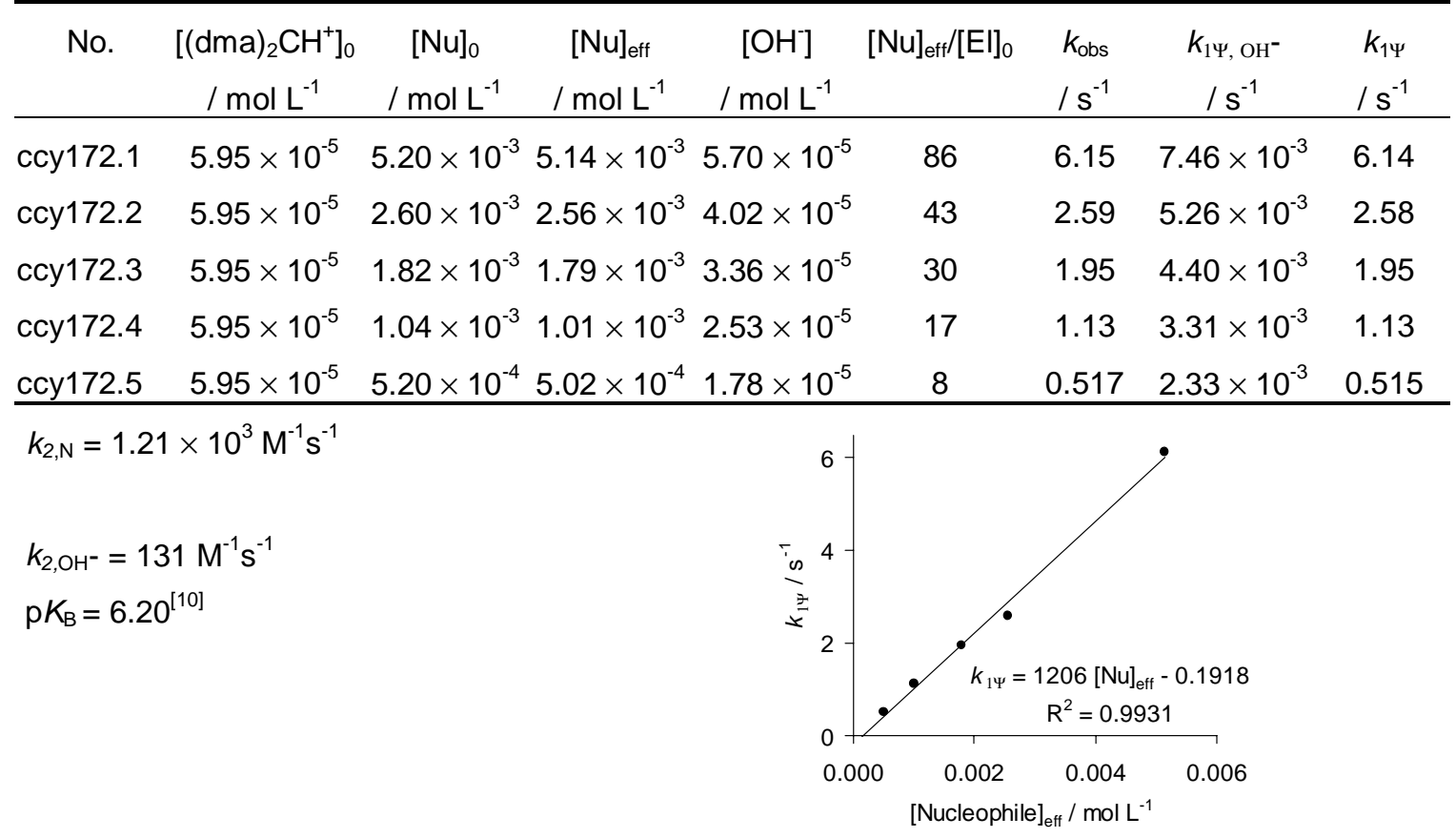

9.1.2. Reaction of methyl glycinate (19) with $(\mathrm{pyr})_{2} \mathrm{CH}^{+} \mathrm{BF}_{4}$ : (at $20{ }^{\circ} \mathrm{C}$, cosolvent: 0.1 vol- $\% \mathrm{CH}_{3} \mathrm{CN}$, stopped-flow, detection at $610 \mathrm{~nm}$ )

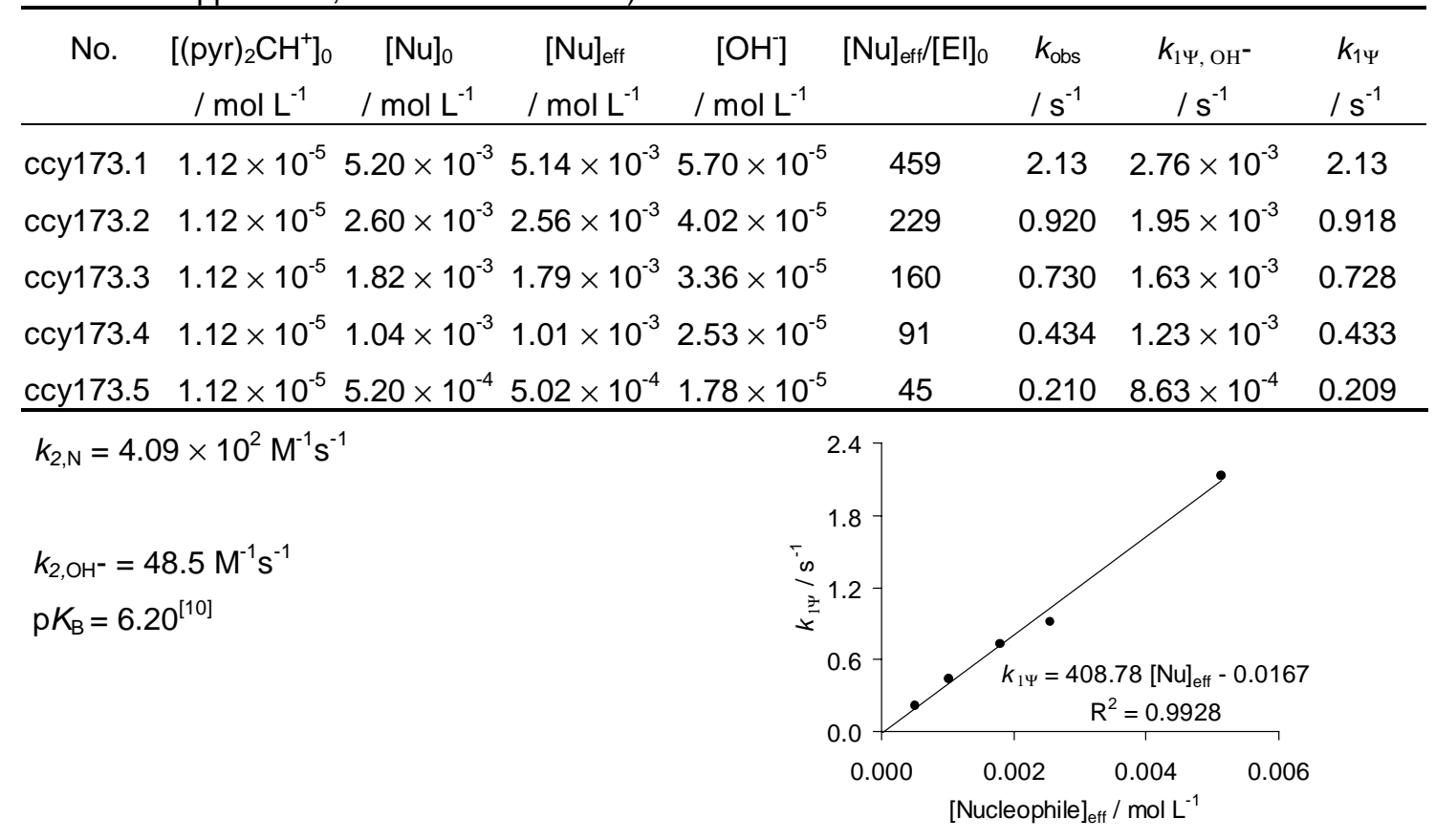


9.1.3. Reaction of methyl glycinate (1g) with (thq) $)_{2} \mathrm{CH}^{+} \mathrm{BF}_{4}^{-}$: (at $20{ }^{\circ} \mathrm{C}$, cosolvent: 0.8 vol- $\% \mathrm{CH}_{3} \mathrm{CN}$, stopped-flow, detection at $610 \mathrm{~nm})$

\begin{tabular}{|c|c|c|c|c|c|c|c|c|}
\hline No. & $\begin{array}{c}{\left[(\text { thq })_{2} \mathrm{CH}^{+}\right]_{0}} \\
/ \mathrm{mol} \mathrm{L}^{-1}\end{array}$ & $\begin{array}{c}{[\mathrm{Nu}]_{0}} \\
/ \mathrm{mol} \mathrm{L}^{-1}\end{array}$ & $\begin{array}{c}{[\mathrm{Nu}]_{\mathrm{eff}}} \\
/ \mathrm{mol} \mathrm{L}^{-1}\end{array}$ & $\begin{array}{c}{\left[\mathrm{OH}^{-}\right]} \\
/ \mathrm{mol} \mathrm{L}^{-1}\end{array}$ & {$[\mathrm{Nu}]_{\mathrm{eff}}\left[[\mathrm{El}]_{0}\right.$} & $\begin{array}{l}k_{\mathrm{obs}} \\
/ \mathrm{s}^{-1}\end{array}$ & $\begin{array}{c}k_{1 \Psi, \mathrm{OH}^{-}} \\
1 \mathrm{~s}^{-1} \\
\end{array}$ & $\begin{array}{l}k_{1 \Psi} \\
/ \mathrm{s}^{-1}\end{array}$ \\
\hline cсy 174.1 & $6.28 \times 10^{-5}$ & $5.20 \times 10^{-3}$ & $5.14 \times 10^{-3}$ & $5.70 \times 10^{-5}$ & 82 & 1.07 & $1.34 \times 10^{-3}$ & 1.07 \\
\hline cсy 174.2 & $6.28 \times 10^{-5}$ & $2.60 \times 10^{-3}$ & $2.56 \times 10^{-3}$ & $4.02 \times 10^{-5}$ & 41 & 0.475 & $9.48 \times 10^{-4}$ & 0.474 \\
\hline сcy 174.3 & $6.28 \times 10^{-5}$ & $1.82 \times 10^{-3}$ & $1.79 \times 10^{-3}$ & $3.36 \times 10^{-5}$ & 28 & 0.375 & $7.92 \times 10^{-4}$ & 0.374 \\
\hline сcy 174.4 & $6.28 \times 10^{-5}$ & $1.04 \times 10^{-3}$ & $1.01 \times 10^{-3}$ & $2.53 \times 10^{-5}$ & 16 & 0.227 & $5.97 \times 10^{-4}$ & 0.226 \\
\hline cсу 174.5 & $6.28 \times 10^{-5}$ & $5.20 \times 10^{-4}$ & $5.02 \times 10^{-4}$ & $1.78 \times 10^{-5}$ & 8 & 0.111 & $4.20 \times 10^{-4}$ & 0.111 \\
\hline
\end{tabular}

$k_{2, \mathrm{~N}}=2.04 \times 10^{2} \mathrm{M}^{-1} \mathrm{~s}^{-1}$

$k_{2, \mathrm{OH}^{-}}=23.6 \mathrm{M}^{-1} \mathrm{~s}^{-1}$

$\mathrm{p} K_{\mathrm{B}}=6.20^{[10]}$

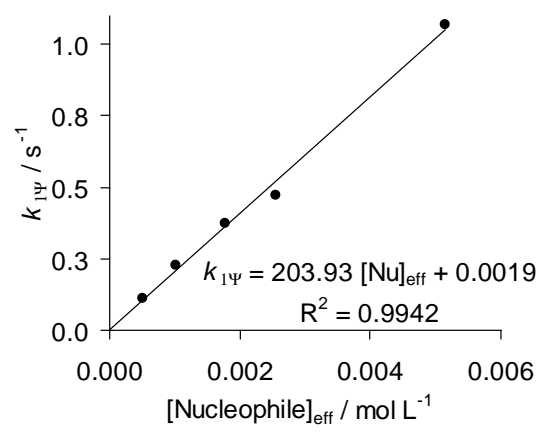

9.1.4. Reaction of methyl glycinate $(\mathbf{1 g})$ with $(\mathrm{jul})_{2} \mathrm{CH}^{+} \mathrm{BF}_{4}$ : (at $20^{\circ} \mathrm{C}$, cosolvent: 0.2 vol- $\% \mathrm{CH}_{3} \mathrm{CN}$, stopped-flow, detection at $634 \mathrm{~nm}$ )

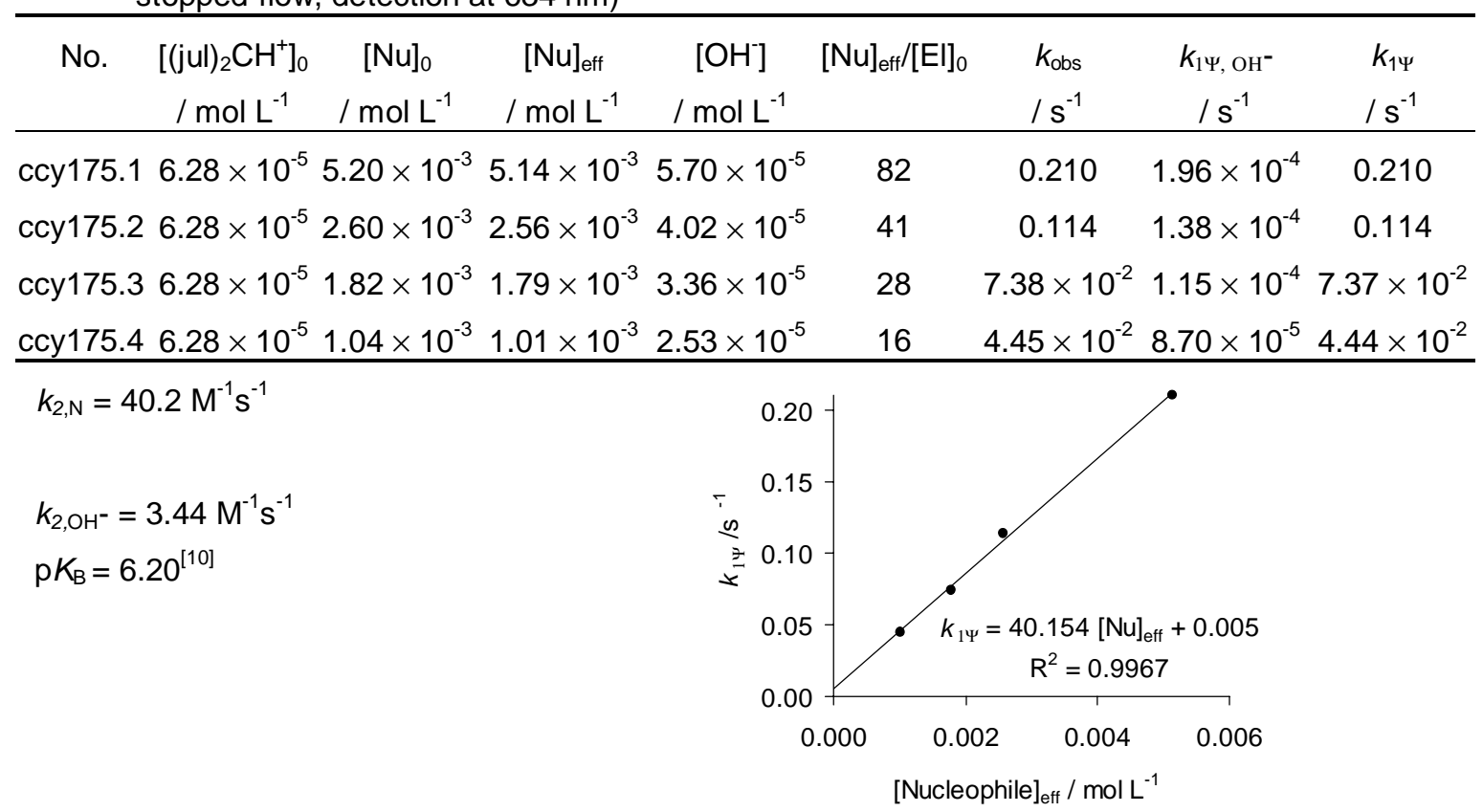


9.2 .

Reactivity parameters in water: $N=12.08 ; s=0.60$

\begin{tabular}{ccc}
\hline Reference electrophile & $E$ parameter & $k_{2, \mathrm{~N}}\left(20^{\circ} \mathrm{C}\right) / \mathrm{M}^{-1} \mathrm{~s}^{-1}$ \\
\hline$(\mathrm{dma})_{2} \mathrm{CH}^{+}$ & -7.02 & $1.21 \times 10^{3}$ \\
$(\mathrm{pyr})_{2} \mathrm{CH}^{+}$ & -7.69 & $4.09 \times 10^{2}$ \\
$(\mathrm{thq})_{2} \mathrm{CH}^{+}$ & -8.22 & $2.04 \times 10^{2}$ \\
$(\mathrm{jul})_{2} \mathrm{CH}^{+}$ & -9.45 & 40.2 \\
\hline
\end{tabular}

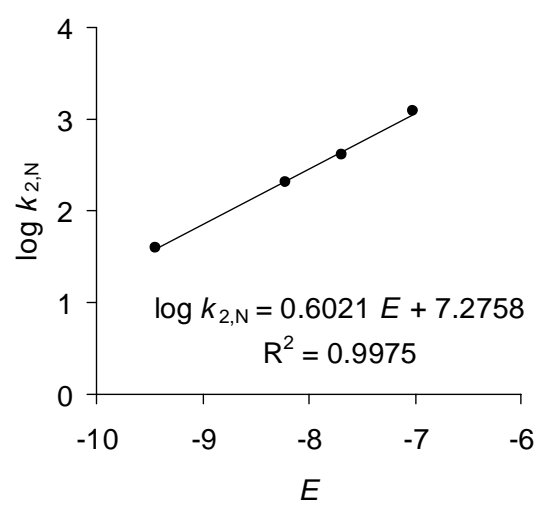

\section{Glycinamide (1h)}

10.1. Rate constants in water

10.1.1. Reaction of glycinamide (1h) with (mor) ${ }_{2} \mathrm{CH}^{+} \mathrm{BF}_{4}{ }_{4}$ : (at $20^{\circ} \mathrm{C}$, cosolvent: 9 vol- $\% \mathrm{CH}_{3} \mathrm{CN}$, stopped-flow, detection at $607 \mathrm{~nm}$ )

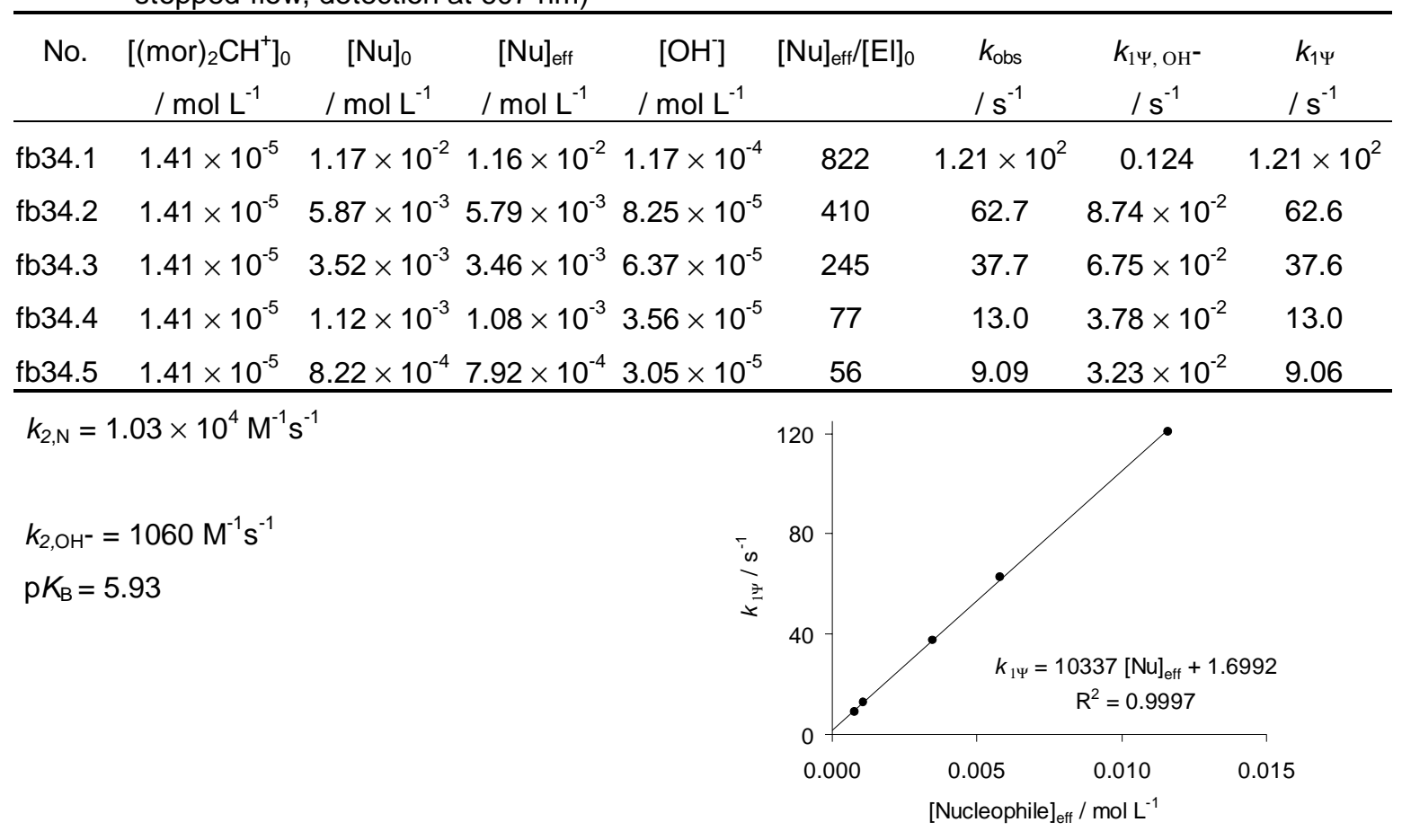


10.1.2. Reaction of glycinamide (1h) with $(\mathrm{dma})_{2} \mathrm{CH}^{+} \mathrm{BF}_{4}{ }_{4}$ : (at $20{ }^{\circ} \mathrm{C}$, cosolvent: 0.4 vol- $\% \mathrm{CH}_{3} \mathrm{CN}$, stopped-flow, detection at $604 \mathrm{~nm}$ )

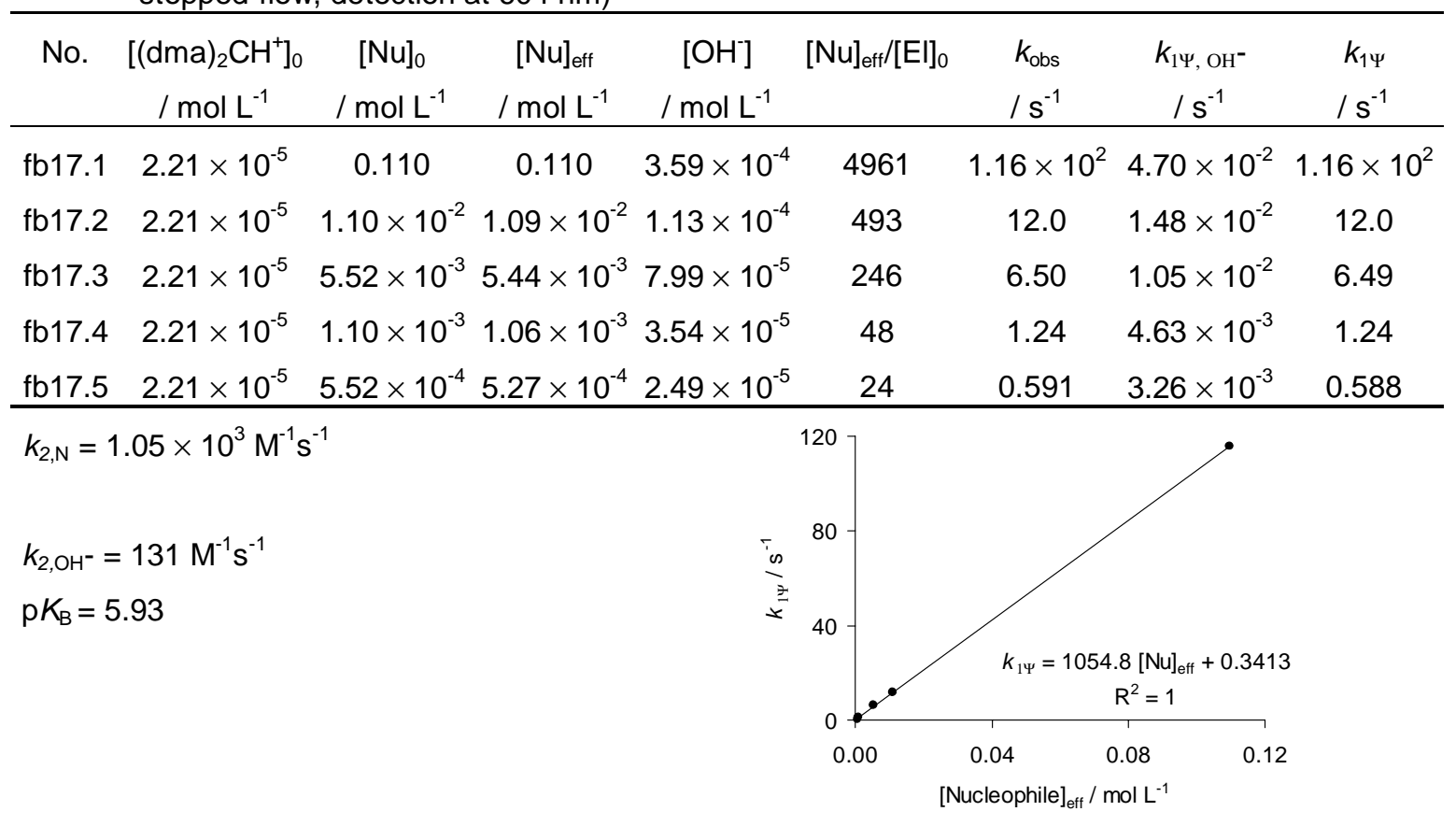

10.1.3. Reaction of glycinamide (1h) with (pyr) $)_{2} \mathrm{CH}^{+} \mathrm{BF}_{4}^{-}$: (at $20^{\circ} \mathrm{C}$, cosolvent: 0.1 vol-\% $\mathrm{CH}_{3} \mathrm{CN}$, stopped-flow, detection at $615 \mathrm{~nm}$ )

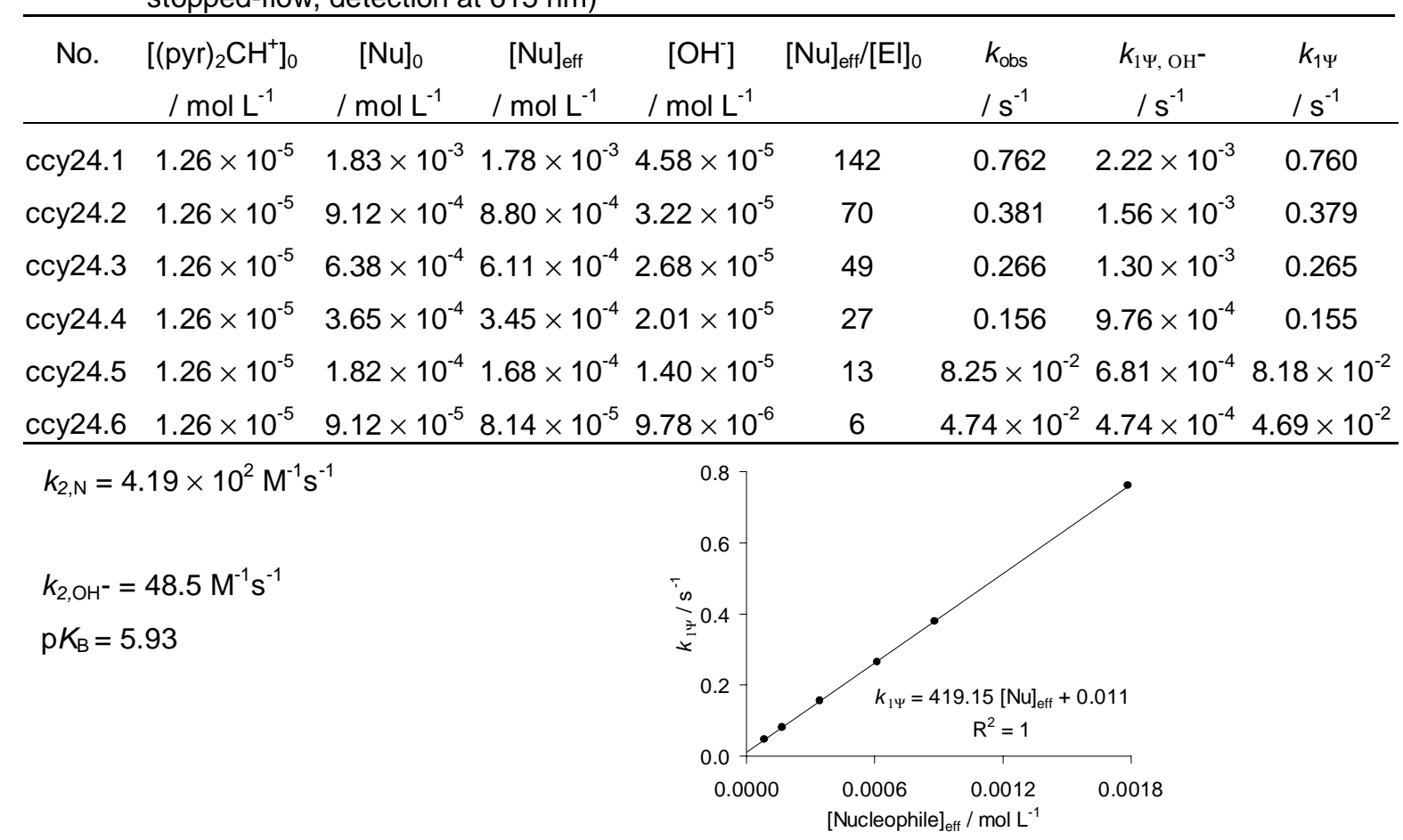


10.1.4. Reaction of glycinamide (1h) with (thq) ${ }_{2} \mathrm{CH}^{+} \mathrm{BF}_{4}^{-}$: (at $20^{\circ} \mathrm{C}$, cosolvent: 0.2 vol-\% $\mathrm{CH}_{3} \mathrm{CN}$, stopped-flow, detection at $615 \mathrm{~nm}$ )

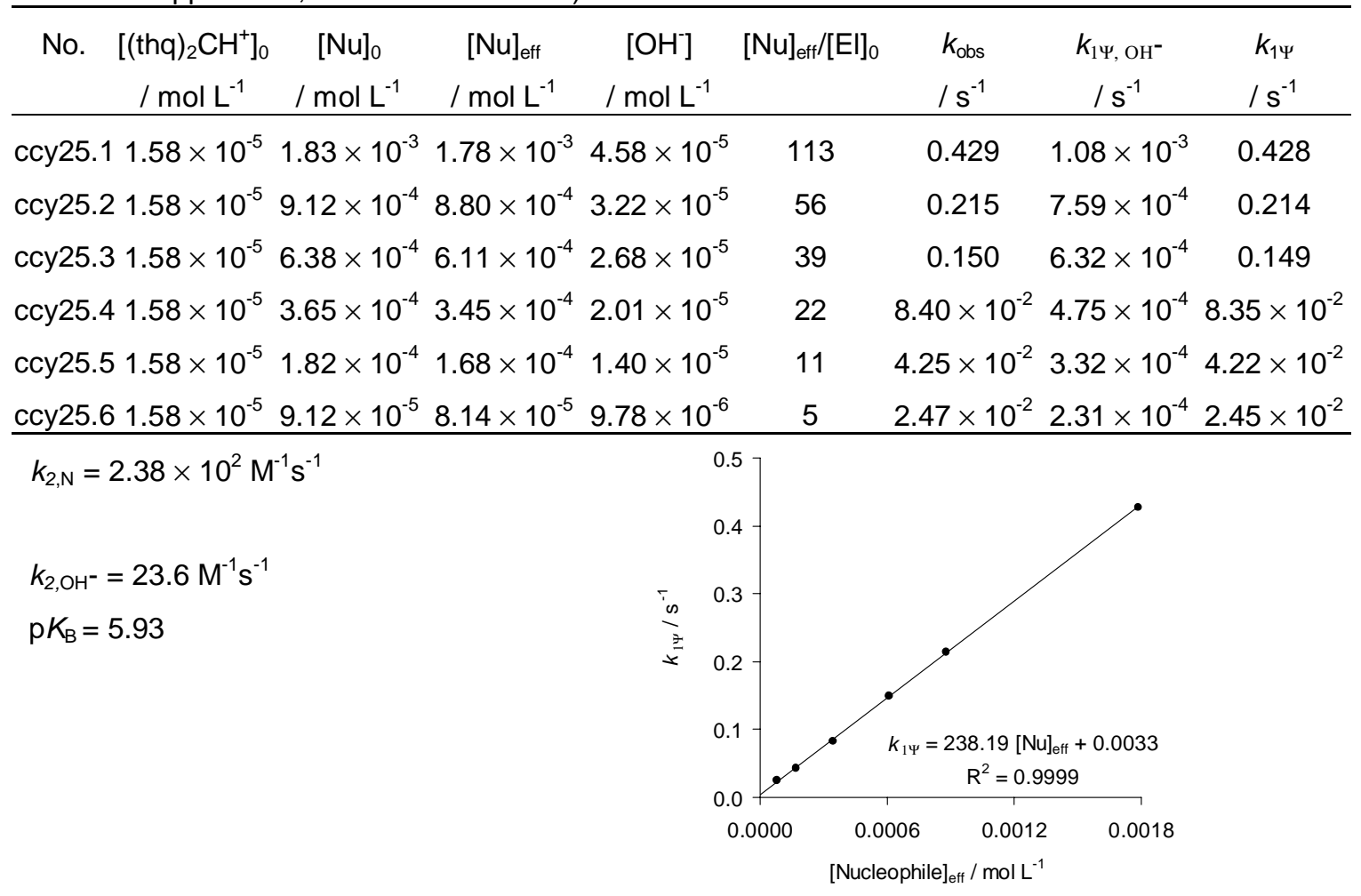

10.1.5. Reaction of glycinamide (1h) with (ind) ${ }_{2} \mathrm{CH}^{+} \mathrm{BF}_{4}$ : (at $20^{\circ} \mathrm{C}$, cosolvent: 0.4 vol-\% $\mathrm{CH}_{3} \mathrm{CN}$, stopped-flow, detection at $614 \mathrm{~nm}$ )

\begin{tabular}{|c|c|c|c|c|c|c|c|c|}
\hline No. & $\begin{array}{c}{\left[(\text { ind })_{2} \mathrm{CH}^{+}\right]_{0}} \\
/ \mathrm{mol} \mathrm{L}^{-1}\end{array}$ & $\begin{array}{c}{[\mathrm{Nu}]_{0}} \\
/ \mathrm{mol} \mathrm{L}^{-1}\end{array}$ & $\begin{array}{c}{[\mathrm{Nu}]_{\mathrm{eff}}} \\
/ \mathrm{mol} \mathrm{L}^{-1}\end{array}$ & $\begin{array}{c}{\left[\mathrm{OH}^{-}\right]} \\
/ \mathrm{mol} \mathrm{L}^{-1}\end{array}$ & {$[\mathrm{Nu}]_{\mathrm{eff}} /[\mathrm{El}]_{0}$} & $\begin{array}{l}k_{\mathrm{obs}} \\
/ \mathrm{s}^{-1}\end{array}$ & $\begin{array}{c}k_{1 \Psi, \mathrm{OH}^{-}} \\
/ \mathrm{s}^{-1}\end{array}$ & $\begin{array}{l}k_{1 \Psi} \\
/ s^{-1}\end{array}$ \\
\hline fb14.1 & $1.39 \times 10^{-5}$ & 0.177 & 0.177 & $4.55 \times 10^{-4}$ & 12700 & 16.3 & $4.92 \times 10^{-3}$ & 16.3 \\
\hline $\mathrm{fb} 14.2$ & $1.39 \times 10^{-5}$ & $1.77 \times 10^{-2}$ & $1.76 \times 10^{-2}$ & $1.44 \times 10^{-4}$ & 1260 & 1.65 & $1.55 \times 10^{-3}$ & 1.65 \\
\hline fb14.3 & $1.39 \times 10^{-5}$ & $8.83 \times 10^{-3}$ & $8.73 \times 10^{-3}$ & $1.01 \times 10^{-4}$ & 628 & 0.852 & $1.09 \times 10^{-3}$ & 0.851 \\
\hline $\mathrm{fb} 14.4$ & $1.39 \times 10^{-5}$ & $1.77 \times 10^{-3}$ & $1.72 \times 10^{-3}$ & $4.50 \times 10^{-5}$ & 124 & 0.176 & $4.86 \times 10^{-4}$ & 0.176 \\
\hline $\mathrm{fb} 14.5$ & $1.39 \times 10^{-5}$ & $8.83 \times 10^{-4}$ & $8.51 \times 10^{-4}$ & $3.16 \times 10^{-5}$ & 61 & $8.98 \times 10^{-2}$ & $3.42 \times 10^{-4}$ & $8.95 \times 10^{-2}$ \\
\hline \multicolumn{2}{|c|}{$k_{2, \mathrm{~N}}=92.2 \mathrm{M}^{-1} \mathrm{~s}^{-1}$} & & & 18 & & & & \\
\hline \multirow{2}{*}{\multicolumn{2}{|c|}{$\begin{array}{l}k_{2, \mathrm{OH}^{-}}=10.8 \mathrm{M}^{-1} \mathrm{~s}^{-1} \\
\mathrm{p} K_{\mathrm{B}}=5.93\end{array}$}} & & & & & & & \\
\hline & & & & & & & & \\
\hline \multirow{3}{*}{$\mathrm{p} K_{\mathrm{B}}=$} & & & & & & & & \\
\hline & & & & & 0.05 & 0.10 & 0.20 & \\
\hline & & & & \multicolumn{5}{|c|}{ [Nucleophile $]_{\text {eff }} / \mathrm{mol} \mathrm{L}^{-1}$} \\
\hline
\end{tabular}


10.1.6. Reaction of glycinamide (1 $\mathbf{h}$ ) with $\left(\mathrm{jul}_{2} \mathrm{CH}^{+} \mathrm{BF}_{4}{ }^{-}\right.$: (at $20^{\circ} \mathrm{C}$, cosolvent: 0.2 vol- $\% \mathrm{CH}_{3} \mathrm{CN}$, stopped-flow, detection at $634 \mathrm{~nm}$ )

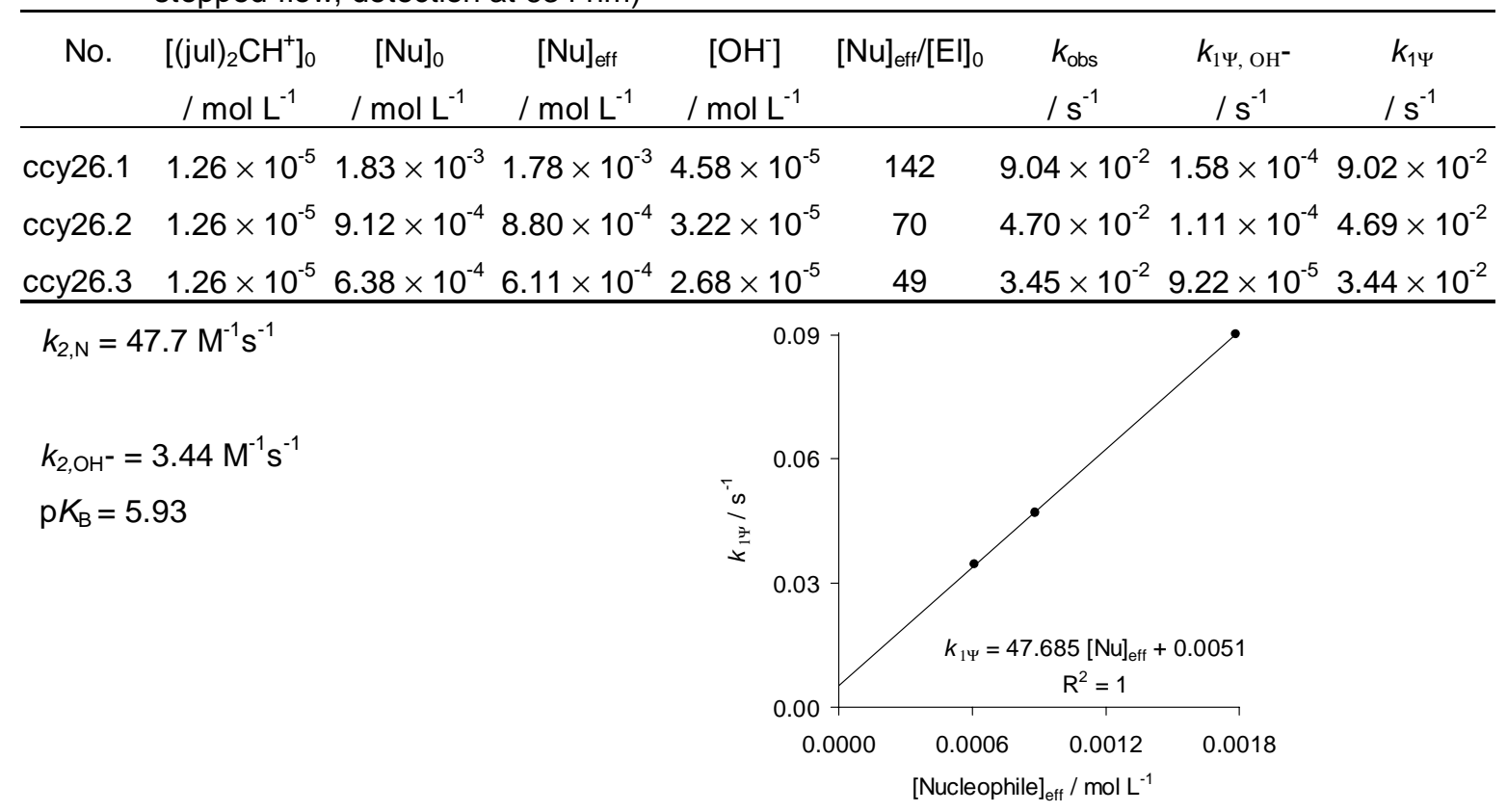

10.1.7. Reaction of glycinamide (1h) with (lil) ${ }_{2} \mathrm{CH}^{+} \mathrm{BF}_{4}{ }^{-}$: (at $20^{\circ} \mathrm{C}$, coslvent: 0.2 vol- $\% \mathrm{CH}_{3} \mathrm{CN}$, stopped-flow, detection at $630 \mathrm{~nm}$ )

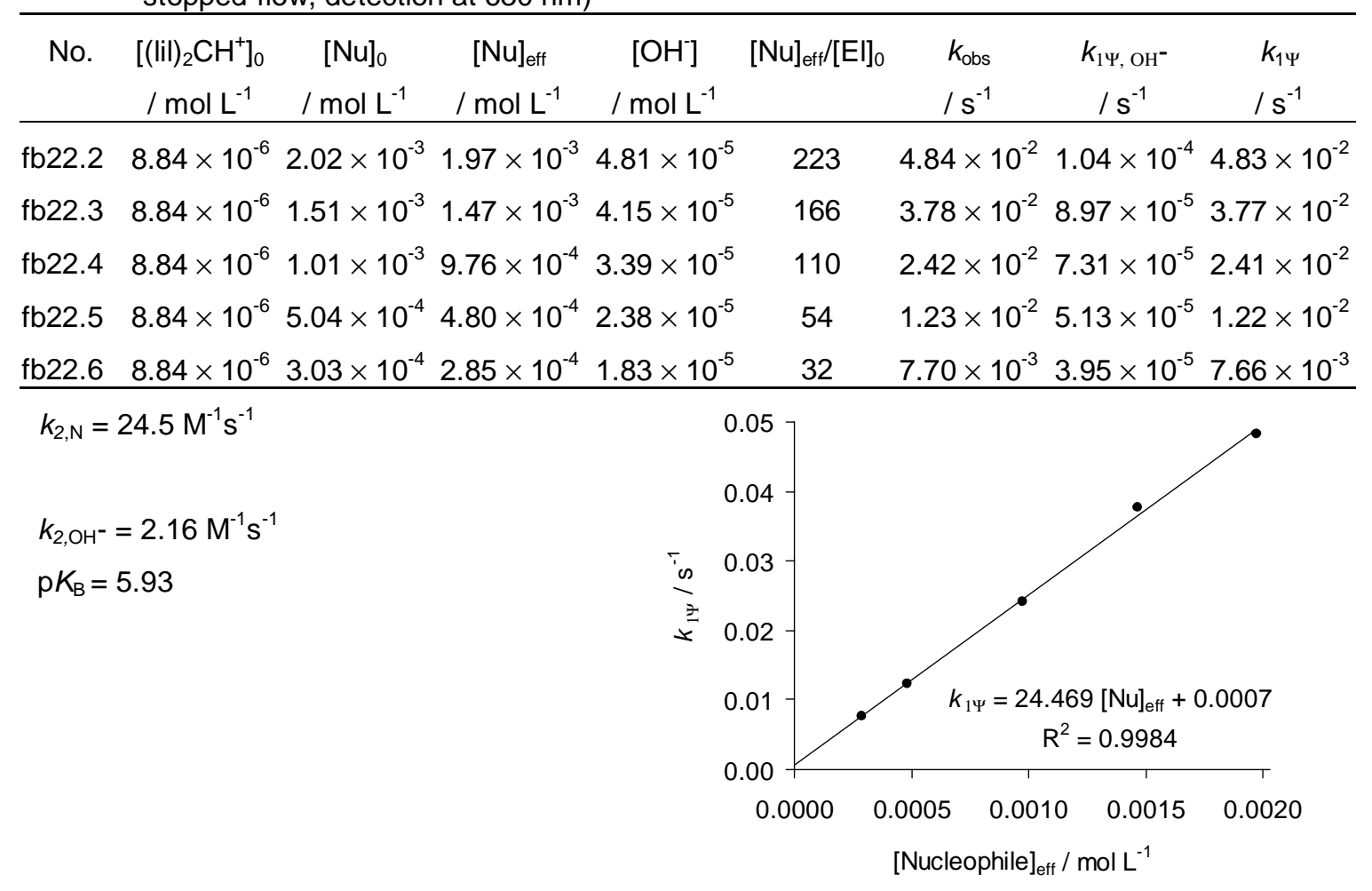


10.2. Reactivity parameters in water: $N=12.29 ; s=0.58$

\begin{tabular}{ccc}
\hline Reference electrophile & $E$ parameter & $k_{2, \mathrm{~N}}\left(20^{\circ} \mathrm{C}\right) / \mathrm{M}^{-1} \mathrm{~s}^{-1}$ \\
\hline$(\mathrm{mor})_{2} \mathrm{CH}^{+}$ & -5.53 & $1.03 \times 10^{4}$ \\
$(\mathrm{dma})_{2} \mathrm{CH}^{+}$ & -7.02 & $1.05 \times 10^{3}$ \\
$(\mathrm{pyr})_{2} \mathrm{CH}^{+}$ & -7.69 & $4.19 \times 10^{2}$ \\
$(\text { thq })_{2} \mathrm{CH}^{+}$ & -8.22 & $2.38 \times 10^{2}$ \\
$(\text { ind })_{2} \mathrm{CH}^{+}$ & -8.76 & 92.2 \\
$(\mathrm{jul})_{2} \mathrm{CH}^{+}$ & -9.45 & 47.7 \\
$(\mathrm{lil})_{2} \mathrm{CH}^{+}$ & -10.04 & 24.5 \\
\hline
\end{tabular}

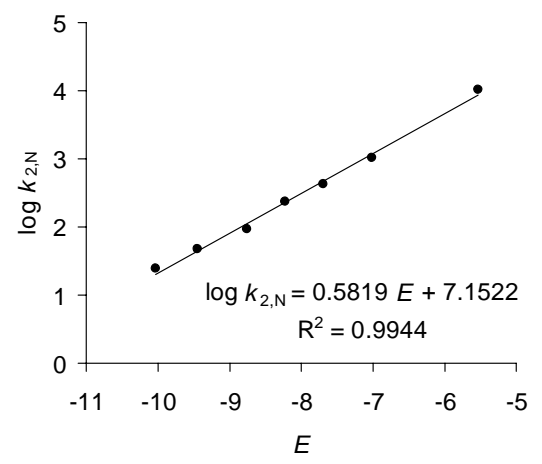

\section{2-Aminoethanol (1i)}

11.1. Rate constants in water

11.1.1. Reaction of 2-Aminoethanol (1i) with (mor) ${ }_{2} \mathrm{CH}^{+} \mathrm{BF}_{4}$ : (at $20^{\circ} \mathrm{C}$, cosolvent: 9 vol- $\% \mathrm{CH}_{3} \mathrm{CN}$, stopped-flow, detection at $607 \mathrm{~nm}$ )

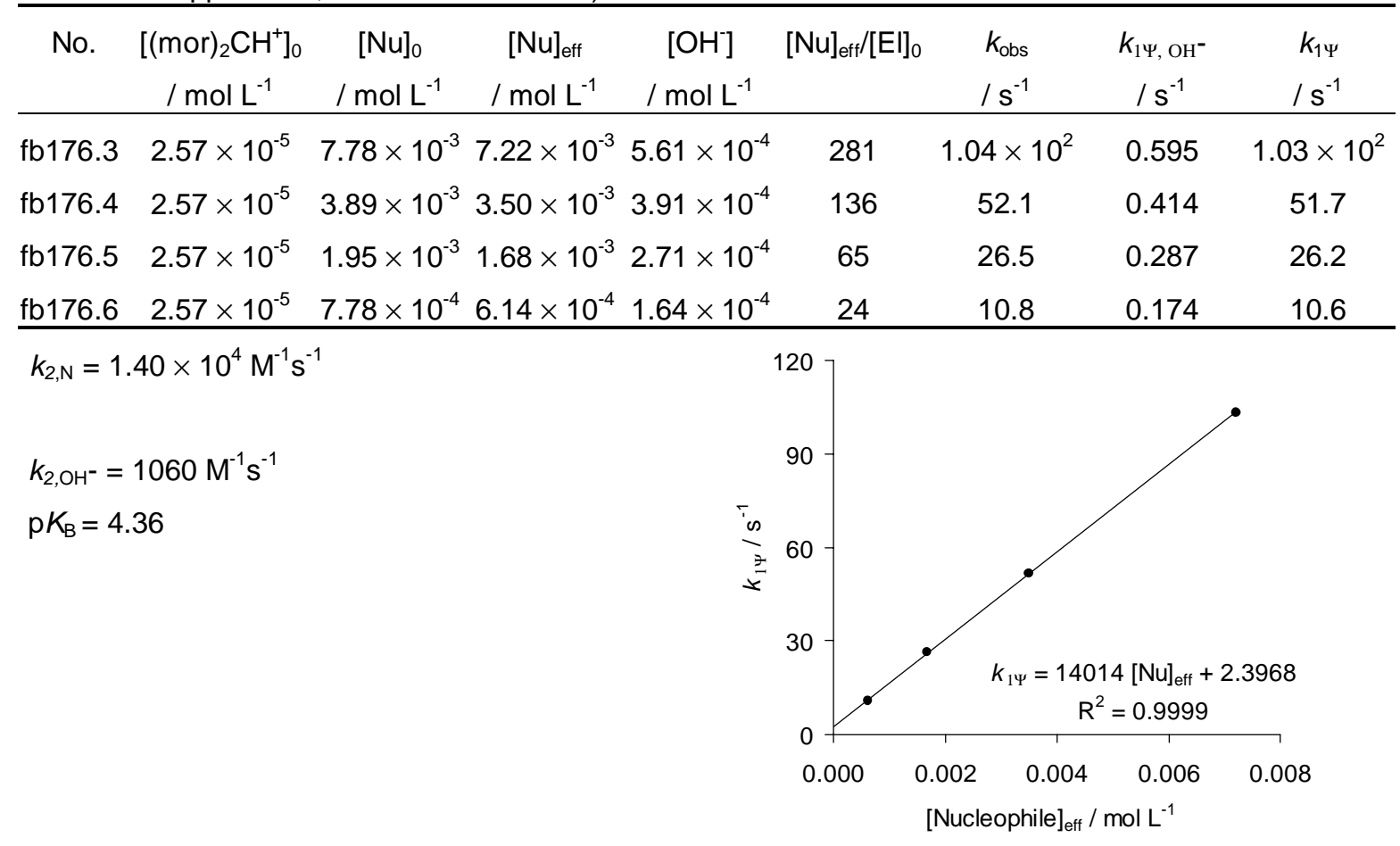


11.1.2. Reaction of 2-Aminoethanol (1i) with $(\mathrm{dma})_{2} \mathrm{CH}^{+} \mathrm{BF}_{4}{ }_{4}$ : (at $20^{\circ} \mathrm{C}$, cosolvent: 0.5 vol- $\% \mathrm{CH}_{3} \mathrm{CN}$, stopped-flow, detection at $610 \mathrm{~nm}$ )

\begin{tabular}{ccccccccc}
\hline No. & $\begin{array}{c}{\left[(\mathrm{dma})_{2} \mathrm{CH}^{+}\right]_{0}} \\
/ \mathrm{mol} \mathrm{L}^{-1}\end{array}$ & $\begin{array}{c}{[\mathrm{Nu}]_{0}} \\
/ \mathrm{mol} \mathrm{L}^{-1}\end{array}$ & $\begin{array}{c}{[\mathrm{Nu}]_{\mathrm{eff}}} \\
/ \mathrm{mol} \mathrm{L}^{-1}\end{array}$ & $\begin{array}{c}{[\mathrm{OH}]} \\
/ \mathrm{mol} \mathrm{L}^{-1}\end{array}$ & $\begin{array}{c}{[\mathrm{Nu}]_{\mathrm{eff}} /[\mathrm{El}]_{0}} \\
k_{\mathrm{obs}}\end{array}$ & $\begin{array}{c}k_{1 \Psi, \mathrm{OH}^{-}} \\
/ \mathrm{s}^{-1}\end{array}$ & $\begin{array}{c}k_{1 \Psi} \\
/ \mathrm{s}^{-1}\end{array}$ & $/ \mathrm{s}^{-1}$ \\
\hline ccy130.1 & $5.95 \times 10^{-5}$ & $6.25 \times 10^{-3}$ & $5.75 \times 10^{-3}$ & $5.01 \times 10^{-4}$ & 97 & 9.37 & $6.56 \times 10^{-2}$ & 9.30 \\
ccy130.2 & $5.95 \times 10^{-5}$ & $3.13 \times 10^{-3}$ & $2.78 \times 10^{-3}$ & $3.48 \times 10^{-4}$ & 47 & 4.48 & $4.56 \times 10^{-2}$ & 4.43 \\
ccy130.3 & $5.95 \times 10^{-5}$ & $2.19 \times 10^{-3}$ & $1.90 \times 10^{-3}$ & $2.88 \times 10^{-4}$ & 32 & 3.01 & $3.77 \times 10^{-2}$ & 2.97 \\
ccy130.5 & $5.95 \times 10^{-5}$ & $6.25 \times 10^{-4}$ & $4.80 \times 10^{-4}$ & $1.45 \times 10^{-4}$ & 8 & 0.712 & $1.90 \times 10^{-2}$ & 0.693 \\
\hline
\end{tabular}

$$
\begin{aligned}
& k_{2, \mathrm{~N}}=1.64 \times 10^{3} \mathrm{M}^{-1} \mathrm{~s}^{-1} \\
& k_{2, \mathrm{OH}^{-}}=131 \mathrm{M}^{-1} \mathrm{~s}^{-1} \\
& \mathrm{p} K_{\mathrm{B}}=4.36
\end{aligned}
$$

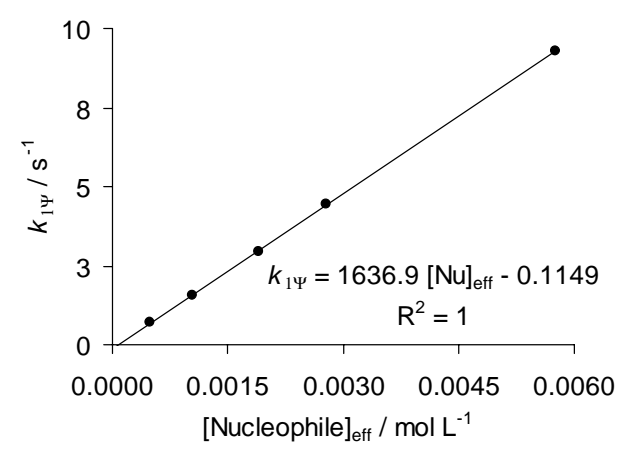

11.1.3. Reaction of 2-Aminoethanol (1i) with $(\mathrm{pyr})_{2} \mathrm{CH}^{+} \mathrm{BF}_{4}$ : (at $20^{\circ} \mathrm{C}$, cosolvent: 0.2 vol- $\% \mathrm{CH}_{3} \mathrm{CN}$, stopped-flow, detection at $610 \mathrm{~nm}$ )

\begin{tabular}{ccccccccc}
\hline No. & $\begin{array}{c}{\left[(\mathrm{pyr})_{2} \mathrm{CH}^{+}\right]_{0}} \\
/ \mathrm{mol} \mathrm{L}^{-1}\end{array}$ & $\begin{array}{c}{[\mathrm{Nu}]_{0}} \\
/ \mathrm{mol} \mathrm{L}^{-1}\end{array}$ & $\begin{array}{c}{[\mathrm{Nu}]_{\mathrm{eff}}} \\
/ \mathrm{mol} \mathrm{L}^{-1}\end{array}$ & $\begin{array}{c}{[\mathrm{OH}]} \\
/ \mathrm{mol} \mathrm{L}^{-1}\end{array}$ & $\begin{array}{c}{[\mathrm{Nu}]_{\mathrm{eff}}[\mathrm{El}]_{0}} \\
\mathrm{k}_{\mathrm{obs}}\end{array}$ & $\begin{array}{c}k_{1 \Psi, \mathrm{OH}^{-}} \\
/ \mathrm{s}^{-1}\end{array}$ & $\begin{array}{c}k_{1 \Psi} \\
/ \mathrm{s}^{-1}\end{array}$ & $/ \mathrm{s}^{-1}$ \\
\hline ccy131.1 & $2.12 \times 10^{-5}$ & $6.25 \times 10^{-3}$ & $5.75 \times 10^{-3}$ & $5.01 \times 10^{-4}$ & 271 & 3.73 & $2.43 \times 10^{-2}$ & 3.71 \\
ccy131.2 & $2.12 \times 10^{-5}$ & $3.13 \times 10^{-3}$ & $2.78 \times 10^{-3}$ & $3.48 \times 10^{-4}$ & 131 & 1.86 & $1.69 \times 10^{-2}$ & 1.84 \\
ccy131.3 & $2.12 \times 10^{-5}$ & $2.19 \times 10^{-3}$ & $1.90 \times 10^{-3}$ & $2.88 \times 10^{-4}$ & 90 & 1.29 & $1.40 \times 10^{-2}$ & 1.28 \\
ccy131.4 & $2.12 \times 10^{-5}$ & $1.25 \times 10^{-3}$ & $1.04 \times 10^{-3}$ & $2.13 \times 10^{-4}$ & 49 & 0.722 & $1.03 \times 10^{-2}$ & 0.712 \\
ccy131.5 & $2.12 \times 10^{-5}$ & $6.25 \times 10^{-4}$ & $4.80 \times 10^{-4}$ & $1.45 \times 10^{-4}$ & 23 & 0.345 & $7.02 \times 10^{-3}$ & 0.338 \\
\hline
\end{tabular}

$$
\begin{aligned}
& k_{2, \mathrm{~N}}=6.38 \times 10^{2} \mathrm{M}^{-1} \mathrm{~s}^{-1} \\
& k_{2, \mathrm{OH}}=48.5 \mathrm{M}^{-1} \mathrm{~s}^{-1} \\
& \mathrm{p} K_{\mathrm{B}}=4.36
\end{aligned}
$$

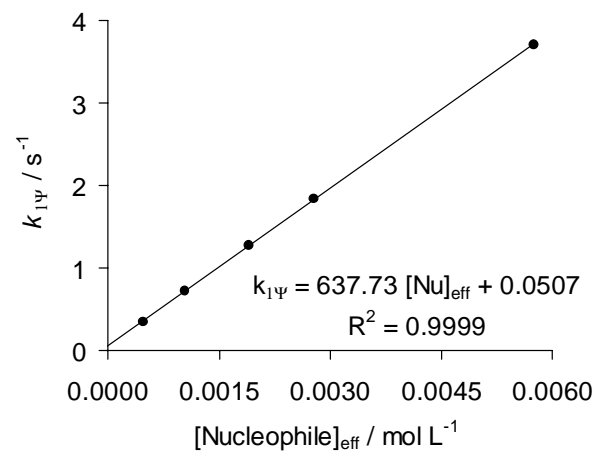


11.1.4. Reaction of 2-Aminoethanol (1i) with (thq) ${ }_{2} \mathrm{CH}^{+} \mathrm{BF}_{4}^{-}$: (at $20^{\circ} \mathrm{C}$, cosolvent: 0.4 vol- $\% \mathrm{CH}_{3} \mathrm{CN}$, stopped-flow, detection at $610 \mathrm{~nm}$ )

\begin{tabular}{|c|c|c|c|c|c|c|c|c|}
\hline No. & $\begin{array}{c}{\left[(\text { thq })_{2} \mathrm{CH}^{+}\right]_{0}} \\
/ \mathrm{mol} \mathrm{L}^{-1}\end{array}$ & $\begin{array}{c}{[\mathrm{Nu}]_{0}} \\
/ \mathrm{mol} \mathrm{L}^{-1}\end{array}$ & $\begin{array}{c}{[\mathrm{Nu}]_{\mathrm{eff}}} \\
/ \mathrm{mol} \mathrm{L}^{-1}\end{array}$ & $\begin{array}{c}{\left[\mathrm{OH}^{-}\right]} \\
/ \mathrm{mol} \mathrm{L}^{-1}\end{array}$ & {$[\mathrm{Nu}]_{\mathrm{efff}} /[\mathrm{El}]_{0}$} & $\begin{array}{l}k_{\mathrm{obs}} \\
/ \mathrm{s}^{-1}\end{array}$ & $\begin{array}{c}k_{1 \Psi, \mathrm{OH}^{-}} \\
/ \mathrm{s}^{-1}\end{array}$ & $\begin{array}{l}k_{1 \Psi} \\
/ \mathrm{s}^{-1}\end{array}$ \\
\hline ccy132.1 & $3.14 \times 10^{-5}$ & $6.25 \times 10^{-3}$ & $5.75 \times 10^{-3}$ & $5.01 \times 10^{-4}$ & 183 & 1.88 & $1.18 \times 10^{-2}$ & 1.87 \\
\hline ccy132.2 & $3.14 \times 10^{-5}$ & $3.13 \times 10^{-3}$ & $2.78 \times 10^{-3}$ & $3.48 \times 10^{-4}$ & 89 & 0.930 & $8.22 \times 10^{-3}$ & 0.922 \\
\hline ccy132.3 & $3.14 \times 10^{-5}$ & $2.19 \times 10^{-3}$ & $1.90 \times 10^{-3}$ & $2.88 \times 10^{-4}$ & 61 & 0.639 & $6.80 \times 10^{-3}$ & 0.632 \\
\hline ccy 132.4 & $3.14 \times 10^{-5}$ & $1.25 \times 10^{-3}$ & $1.04 \times 10^{-3}$ & $2.13 \times 10^{-4}$ & 33 & 0.353 & $5.02 \times 10^{-3}$ & 0.348 \\
\hline ccy 132.5 & $3.14 \times 10^{-5}$ & $6.25 \times 10^{-4}$ & $4.80 \times 10^{-4}$ & $1.45 \times 10^{-4}$ & 15 & 0.165 & $3.42 \times 10^{-3}$ & 0.162 \\
\hline
\end{tabular}

$$
\begin{aligned}
& k_{2, \mathrm{~N}}=3.23 \times 10^{2} \mathrm{M}^{-1} \mathrm{~s}^{-1} \\
& k_{2, \mathrm{OH}^{-}}=23.6 \mathrm{M}^{-1} \mathrm{~s}^{-1} \\
& \mathrm{p} K_{\mathrm{B}}=4.36
\end{aligned}
$$

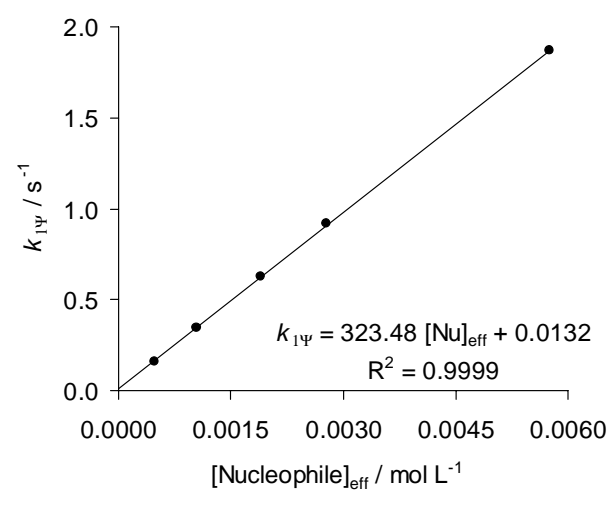

11.1.5. Reaction of 2-Aminoethanol (1i) with (jul) ${ }_{2} \mathrm{CH}^{+} \mathrm{BF}_{4}{ }_{4}$ : (at $20{ }^{\circ} \mathrm{C}$, cosolvent: 0.2 vol- $\% \mathrm{CH}_{3} \mathrm{CN}$, stopped-flow, detection at $634 \mathrm{~nm}$ )

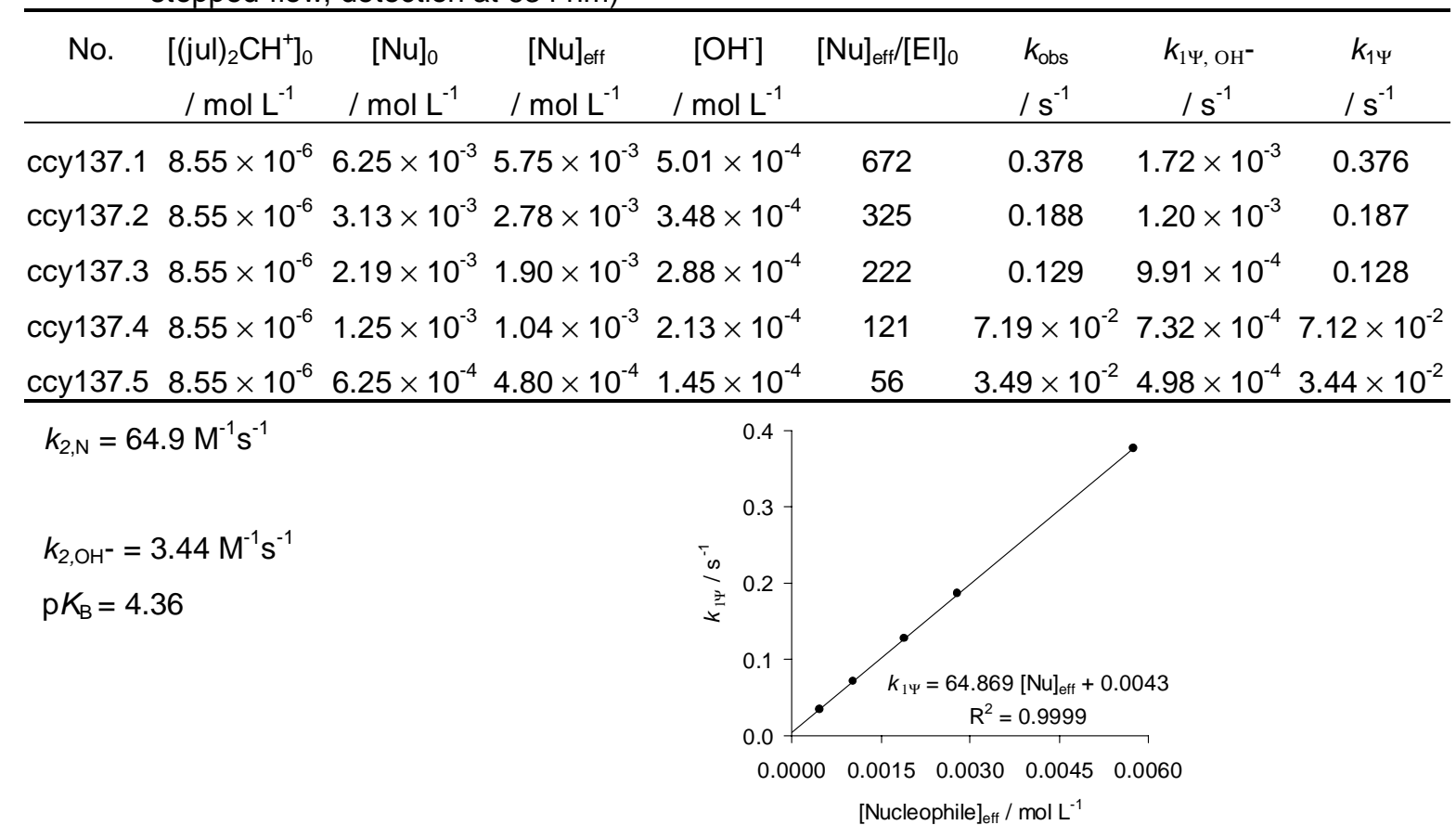


11.1.6. Reaction of 2-Aminoethanol (1i) with (lii) ${ }_{2} \mathrm{CH}^{+} \mathrm{BF}_{4}$ : (at $20^{\circ} \mathrm{C}$, cosolvent: 0.2 vol- $\% \mathrm{CH}_{3} \mathrm{CN}$, stopped-flow, detection at $634 \mathrm{~nm}$ )

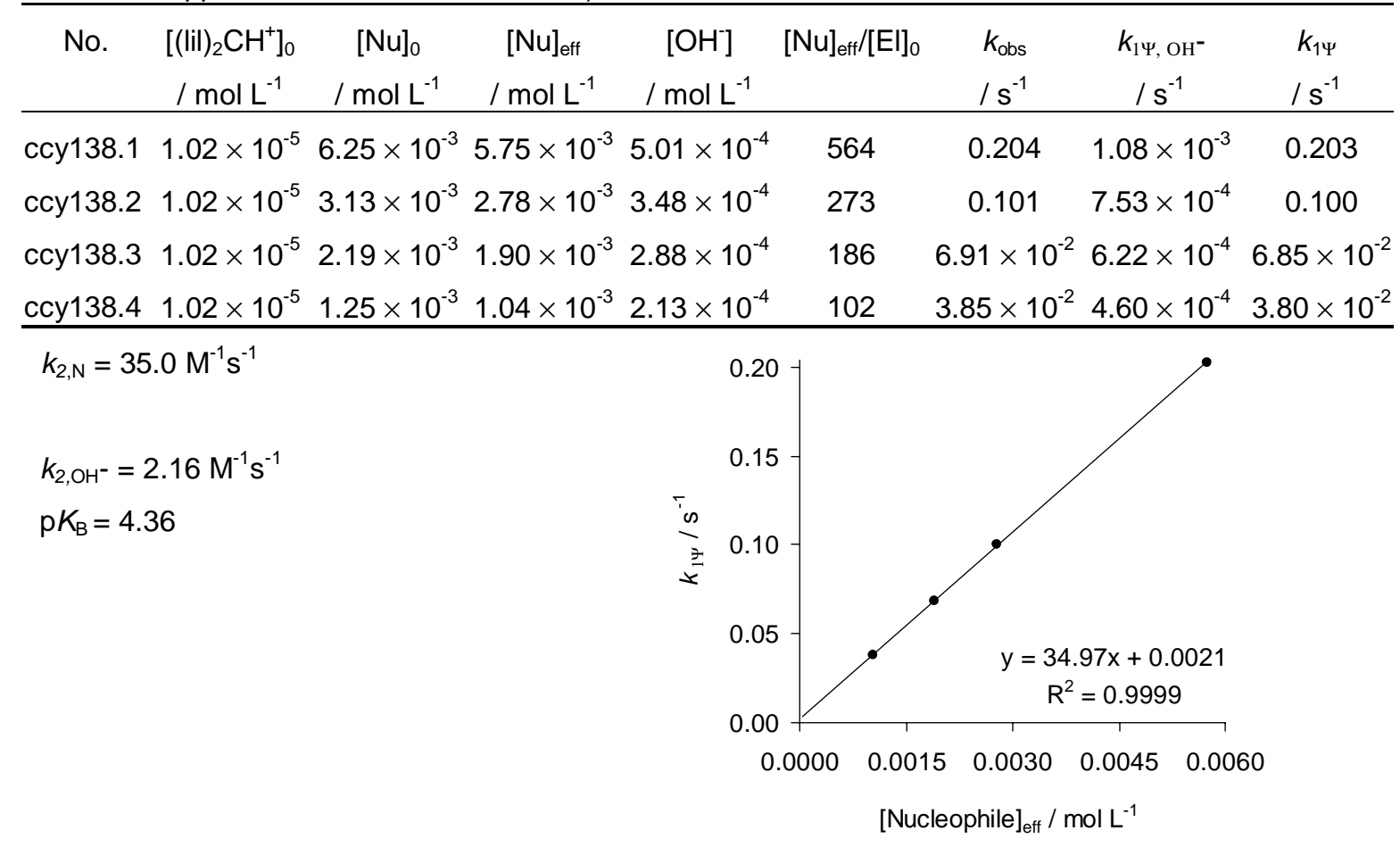

11.2. Reactivity parameters in water: $N=12.61 ; s=0.58$

\begin{tabular}{ccc}
\hline Reference electrophile & $E$ parameter & $k_{2, \mathrm{~N}}\left(20^{\circ} \mathrm{C}\right) / \mathrm{M}^{-1} \mathrm{~s}^{-1}$ \\
\hline (mor $)_{2} \mathrm{CH}^{+}$ & -5.53 & $1.40 \times 10^{4}$ \\
$(\mathrm{dma})_{2} \mathrm{CH}^{+}$ & -7.02 & $1.64 \times 10^{3}$ \\
$(\mathrm{pyr})_{2} \mathrm{CH}^{+}$ & -7.69 & $6.38 \times 10^{2}$ \\
$(\mathrm{thq})_{2} \mathrm{CH}^{+}$ & -8.22 & $3.23 \times 10^{2}$ \\
$(\mathrm{jul})_{2} \mathrm{CH}^{+}$ & -9.45 & 64.9 \\
$(\mathrm{lil})_{2} \mathrm{CH}^{+}$ & -10.04 & 35.0 \\
\hline
\end{tabular}

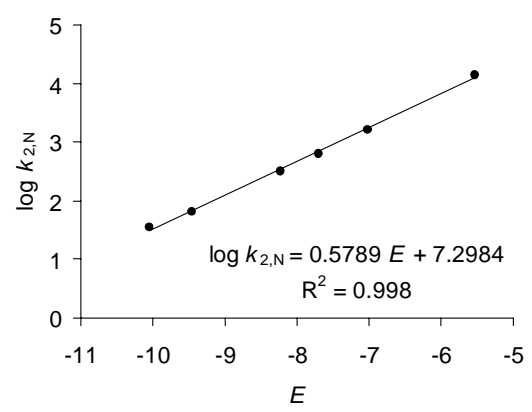




\section{1,2-Ethanediamine (1j)}

12.1. Rate constants in water

12.1.1. Reaction of 1,2-Ethanediamine (1j) with (mor) ${ }_{2} \mathrm{CH}^{+} \mathrm{BF}_{4}$ : (at $20^{\circ} \mathrm{C}$, cosolvent: 9 vol-\% $\mathrm{CH}_{3} \mathrm{CN}$, stopped-flow, detection at $618 \mathrm{~nm}$ )

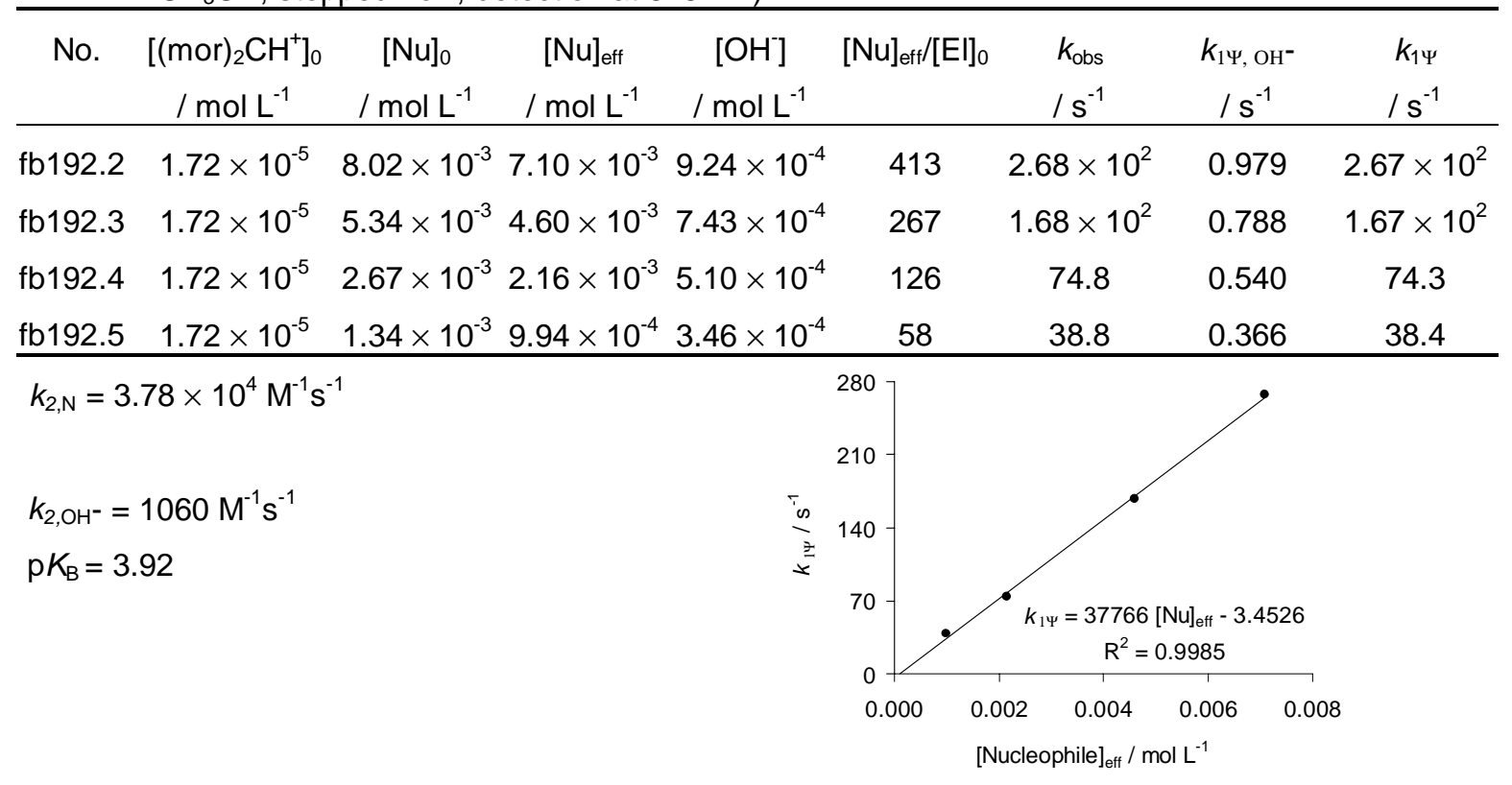

12.1.2. Reaction of 1,2 -Ethanediamine (1j) with $(\mathrm{dma})_{2} \mathrm{CH}^{+} \mathrm{BF}_{4}^{-}$: (at $20^{\circ} \mathrm{C}$, cosolvent: 0.5 vol-\% $\mathrm{CH}_{3} \mathrm{CN}$, stopped-flow, detection at $610 \mathrm{~nm}$ )

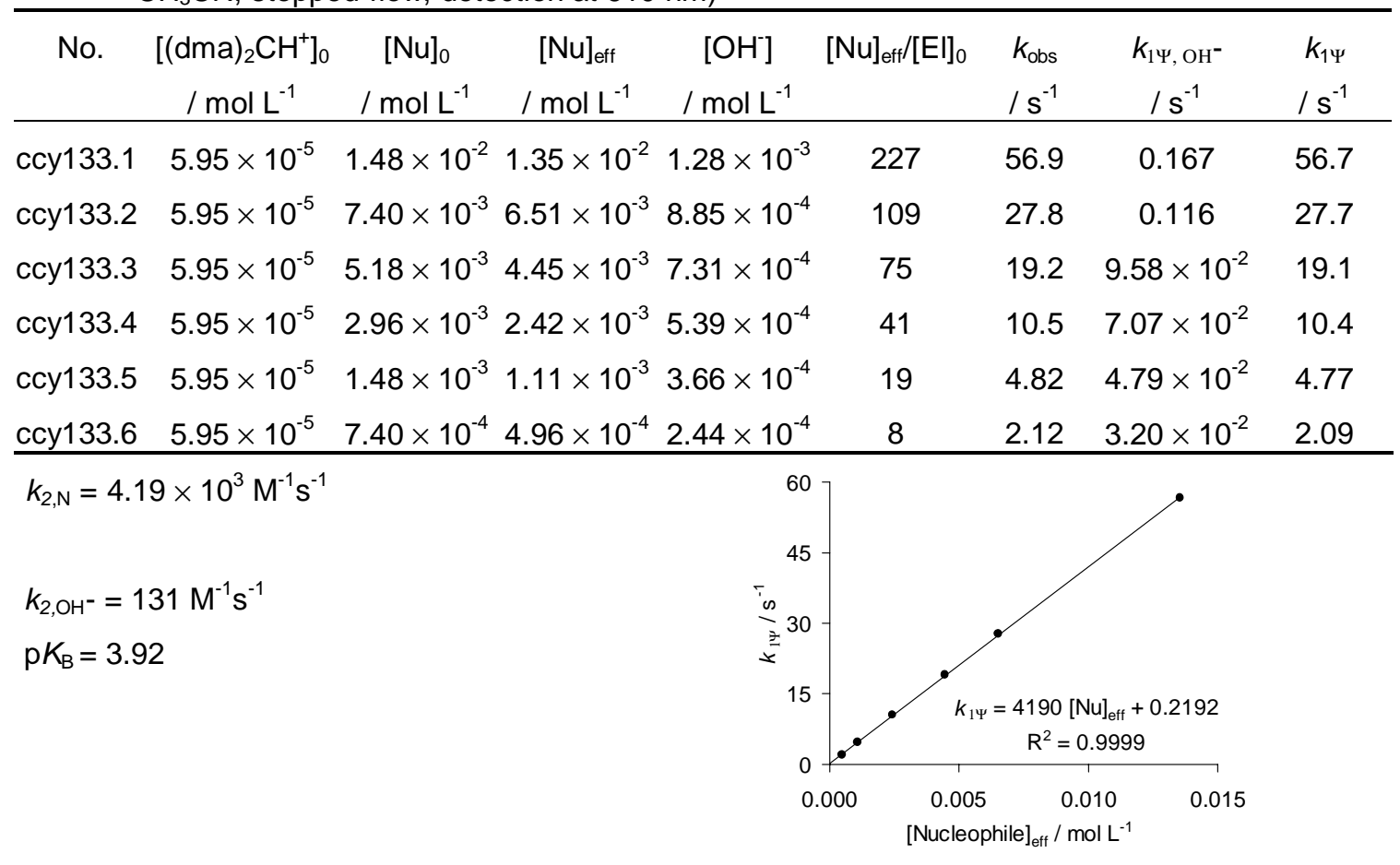


12.1.3. Reaction of 1,2-Ethanediamine (1j) with $(\mathrm{pyr})_{2} \mathrm{CH}^{+} \mathrm{BF}_{4}$ : (at $20^{\circ} \mathrm{C}$, cosolvent: 0.2 vol-\% $\mathrm{CH}_{3} \mathrm{CN}$, stopped-flow, detection at $610 \mathrm{~nm}$ )

\begin{tabular}{ccccccccc}
\hline No. & $\begin{array}{c}{\left[(\mathrm{pyr})_{2} \mathrm{CH}^{+}\right]_{0}} \\
/ \mathrm{mol} \mathrm{L}^{-1}\end{array}$ & $\begin{array}{c}{[\mathrm{Nu}]_{0}} \\
/ \mathrm{mol} \mathrm{L}^{-1}\end{array}$ & $\begin{array}{c}{[\mathrm{Nu}]_{\mathrm{eff}}} \\
/ \mathrm{mol} \mathrm{L}^{-1}\end{array}$ & $\begin{array}{c}{[\mathrm{OH}]} \\
/ \mathrm{mol} \mathrm{L}^{-1}\end{array}$ & $\begin{array}{c}{[\mathrm{Nu}]_{\mathrm{eff}} /[\mathrm{El}]_{0}} \\
k_{\mathrm{obs}}\end{array}$ & $\begin{array}{c}k_{1 \Psi, \mathrm{OH}^{-}} \\
/ \mathrm{s}^{-1}\end{array}$ & $\begin{array}{c}k_{1 \Psi} \\
/ \mathrm{s}^{-1}\end{array}$ & $/ \mathrm{s}^{-1}$ \\
\hline ccy134.1 & $2.12 \times 10^{-5}$ & $1.48 \times 10^{-2}$ & $1.35 \times 10^{-2}$ & $1.28 \times 10^{-3}$ & 638 & 23.1 & $6.18 \times 10^{-2}$ & 23.0 \\
ccy134.2 & $2.12 \times 10^{-5}$ & $7.40 \times 10^{-3}$ & $6.51 \times 10^{-3}$ & $8.85 \times 10^{-4}$ & 307 & 11.1 & $4.29 \times 10^{-2}$ & 11.1 \\
ccy134.3 & $2.12 \times 10^{-5}$ & $5.18 \times 10^{-3}$ & $4.45 \times 10^{-3}$ & $7.31 \times 10^{-4}$ & 210 & 7.66 & $3.55 \times 10^{-2}$ & 7.62 \\
ccy134.4 & $2.12 \times 10^{-5}$ & $2.96 \times 10^{-3}$ & $2.42 \times 10^{-3}$ & $5.39 \times 10^{-4}$ & 114 & 4.25 & $2.62 \times 10^{-2}$ & 4.22 \\
ccy134.5 & $2.12 \times 10^{-5}$ & $1.48 \times 10^{-3}$ & $1.11 \times 10^{-3}$ & $3.66 \times 10^{-4}$ & 53 & 2.06 & $1.77 \times 10^{-2}$ & 2.04 \\
ccy134.6 $2.12 \times 10^{-5}$ & $7.40 \times 10^{-4}$ & $4.96 \times 10^{-4}$ & $2.44 \times 10^{-4}$ & 23 & 0.972 & $1.18 \times 10^{-2}$ & 0.960 \\
\hline
\end{tabular}

$$
\begin{aligned}
& k_{2, \mathrm{~N}}=1.69 \times 10^{3} \mathrm{M}^{-1} \mathrm{~s}^{-1} \\
& k_{2, \mathrm{OH}^{-}}=48.5 \mathrm{M}^{-1} \mathrm{~s}^{-1} \\
& \mathrm{p} K_{\mathrm{B}}=3.92
\end{aligned}
$$

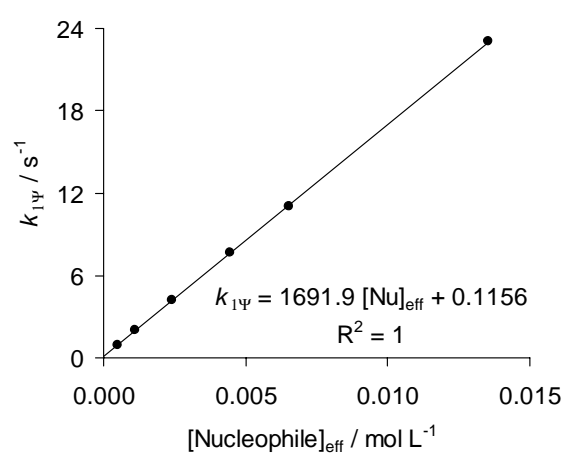

\begin{tabular}{|c|c|c|c|c|c|c|c|c|}
\hline No. & $\begin{array}{c}{\left[(\text { thq })_{2} \mathrm{CH}^{+}\right]_{0}} \\
/ \mathrm{mol} \mathrm{L}^{-1}\end{array}$ & $\begin{array}{c}{[\mathrm{Nu}]_{0}} \\
/ \mathrm{mol} \mathrm{L}^{-1}\end{array}$ & $\begin{array}{c}{[\mathrm{Nu}]_{\mathrm{eff}}} \\
/ \mathrm{mol} \mathrm{L}^{-1}\end{array}$ & $\begin{array}{c}{\left[\mathrm{OH}^{-}\right]} \\
/ \mathrm{mol} \mathrm{L}^{-1}\end{array}$ & [Nul & $\begin{array}{l}k_{\mathrm{obs}} \\
/ \mathrm{s}^{-1}\end{array}$ & $\begin{array}{c}k_{1 \Psi, \mathrm{OH}^{-}} \\
/ \mathrm{s}^{-1}\end{array}$ & $\begin{array}{l}k_{1 \Psi} \\
/ \mathrm{s}^{-1}\end{array}$ \\
\hline 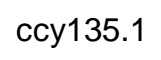 & $3.14 \times 10^{-5}$ & $1.48 \times 10^{-2}$ & $1.35 \times 10^{-2}$ & $1.28 \times 10^{-3}$ & 4 & 11.8 & $3.01 \times 10^{-2}$ & 18 \\
\hline cy135.2 & $3.14 \times 10^{-5}$ & $7.40 \times 10^{-3}$ & $6.51 \times 10^{-3}$ & $8.85 \times 10^{-4}$ & 20 & 5.71 & 2.09 & 5. \\
\hline 53 & $3.14 \times 10^{-5}$ & $5.18 \times 1$ & $4.45 \times 10^{-3}$ & $7.31 \times 10^{-4}$ & 14 & 392 & 1.73 & 3.90 \\
\hline cy135.4 & $3.14 \times 10^{-5}$ & $2.96 \times 10^{-3}$ & $2.42 \times 10^{-3}$ & $5.39 \times 10^{-4}$ & 7 & 2. & $1.27 \times 10^{-2}$ & 2. \\
\hline 5 & $3.14 \times 10^{-5}$ & $1.48 \times 10^{-3}$ & $1.11 \times 10^{-3}$ & $3.66 \times 10^{-4}$ & 3 & 3 & $8.64 \times 10^{-3}$ & 1.02 \\
\hline cy135.6 & $3.14 \times 10^{-5}$ & $7.40 \times 10^{-4}$ & $4.96 \times 10^{-4}$ & $2.44 \times 10^{-4}$ & 16 & 0.474 & $5.76 \times 10^{-3}$ & 0.46 \\
\hline
\end{tabular}

12.1.4. Reaction of 1,2-Ethanediamine (1j) with (thq) $)_{2} \mathrm{CH}^{+} \mathrm{BF}_{4}^{-}$: (at $20^{\circ} \mathrm{C}$, cosolvent: 0.4 vol-\% $\mathrm{CH}_{3} \mathrm{CN}$, stopped-flow, detection at $610 \mathrm{~nm}$ )

$$
\begin{aligned}
& k_{2, \mathrm{~N}}=8.67 \times 10^{2} \mathrm{M}^{-1} \mathrm{~s}^{-1} \\
& k_{2, \mathrm{OH}^{-}}=23.6 \mathrm{M}^{-1} \mathrm{~s}^{-1} \\
& \mathrm{p} K_{\mathrm{B}}=3.92
\end{aligned}
$$

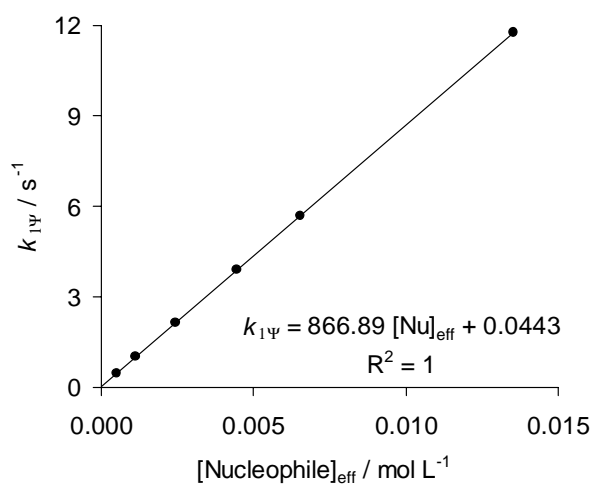


12.1.5. Reaction of 1,2-Ethanediamine (1j) with (ind) ${ }_{2} \mathrm{CH}^{+} \mathrm{BF}_{4}{ }_{4}$ : (at $20^{\circ} \mathrm{C}$, cosolvent: 0.4 vol-\% $\mathrm{CH}_{3} \mathrm{CN}$, stopped-flow, detection at $614 \mathrm{~nm}$ )

\begin{tabular}{ccccccccc}
\hline No. & $\begin{array}{c}{\left[(\mathrm{ind})_{2} \mathrm{CH}^{+}\right]_{0}} \\
/ \mathrm{mol} \mathrm{L}^{-1}\end{array}$ & $\begin{array}{c}{[\mathrm{Nu}]_{0}} \\
/ \mathrm{mol} \mathrm{L}^{-1}\end{array}$ & $\begin{array}{c}{[\mathrm{Nu}]_{\mathrm{eff}}} \\
/ \mathrm{mol} \mathrm{L}^{-1}\end{array}$ & $\begin{array}{c}{[\mathrm{OH}]} \\
/ \mathrm{mol} \mathrm{L}^{-1}\end{array}$ & {$[\mathrm{Nu}]_{\mathrm{eff}} /[\mathrm{El}]_{0}$} & $\begin{array}{c}k_{\mathrm{obs}} \\
/ \mathrm{s}^{-1}\end{array}$ & $\begin{array}{c}k_{1 \Psi, \mathrm{OH}^{-}} \\
/ \mathrm{s}^{-1}\end{array}$ & $\begin{array}{c}k_{1 \Psi} \\
/ \mathrm{s}^{-1}\end{array}$ \\
\hline fb191.1 & $2.34 \times 10^{-5}$ & $7.35 \times 10^{-3}$ & $6.47 \times 10^{-3}$ & $8.82 \times 10^{-4}$ & 276 & 2.48 & $9.52 \times 10^{-3}$ & 2.47 \\
fb191.2 & $2.34 \times 10^{-5}$ & $4.41 \times 10^{-3}$ & $3.74 \times 10^{-3}$ & $6.71 \times 10^{-4}$ & 160 & 1.45 & $7.24 \times 10^{-3}$ & 1.44 \\
fb191.3 & $2.34 \times 10^{-5}$ & $2.94 \times 10^{-3}$ & $2.40 \times 10^{-3}$ & $5.37 \times 10^{-4}$ & 103 & 0.946 & $5.80 \times 10^{-3}$ & 0.940 \\
fb191.4 & $2.34 \times 10^{-5}$ & $1.47 \times 10^{-3}$ & $1.11 \times 10^{-3}$ & $3.65 \times 10^{-4}$ & 47 & 0.447 & $3.94 \times 10^{-3}$ & 0.443 \\
fb191.5 & $2.34 \times 10^{-5}$ & $7.35 \times 10^{-4}$ & $4.92 \times 10^{-4}$ & $2.43 \times 10^{-4}$ & 21 & 0.210 & $2.63 \times 10^{-3}$ & 0.207 \\
\hline
\end{tabular}

$$
\begin{aligned}
& k_{2, \mathrm{~N}}=3.78 \times 10^{2} \mathrm{M}^{-1} \mathrm{~s}^{-1} \\
& k_{2, \mathrm{OH}^{-}}=10.8 \mathrm{M}^{-1} \mathrm{~s}^{-1} \\
& \mathrm{p} K_{\mathrm{B}}=3.92
\end{aligned}
$$

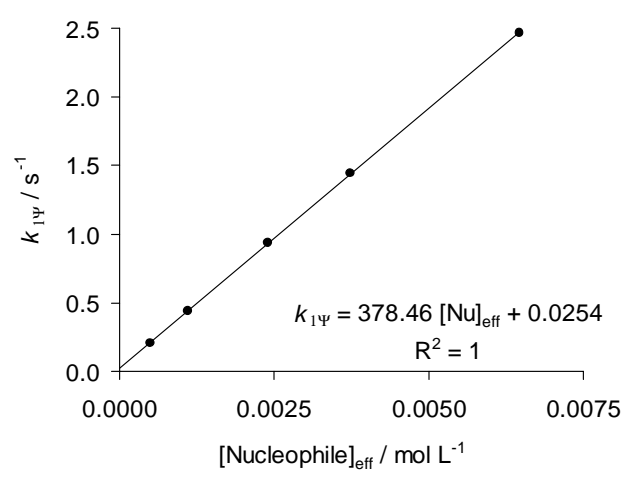

12.1.6. Reaction of 1,2-Ethanediamine (1j) with $(\mathrm{jul})_{2} \mathrm{CH}^{+} \mathrm{BF}_{4}{ }_{4}$ : (at $20^{\circ} \mathrm{C}$, cosolvent: 0.2 vol-\% $\mathrm{CH}_{3} \mathrm{CN}$, stopped-flow, detection at $634 \mathrm{~nm}$ )

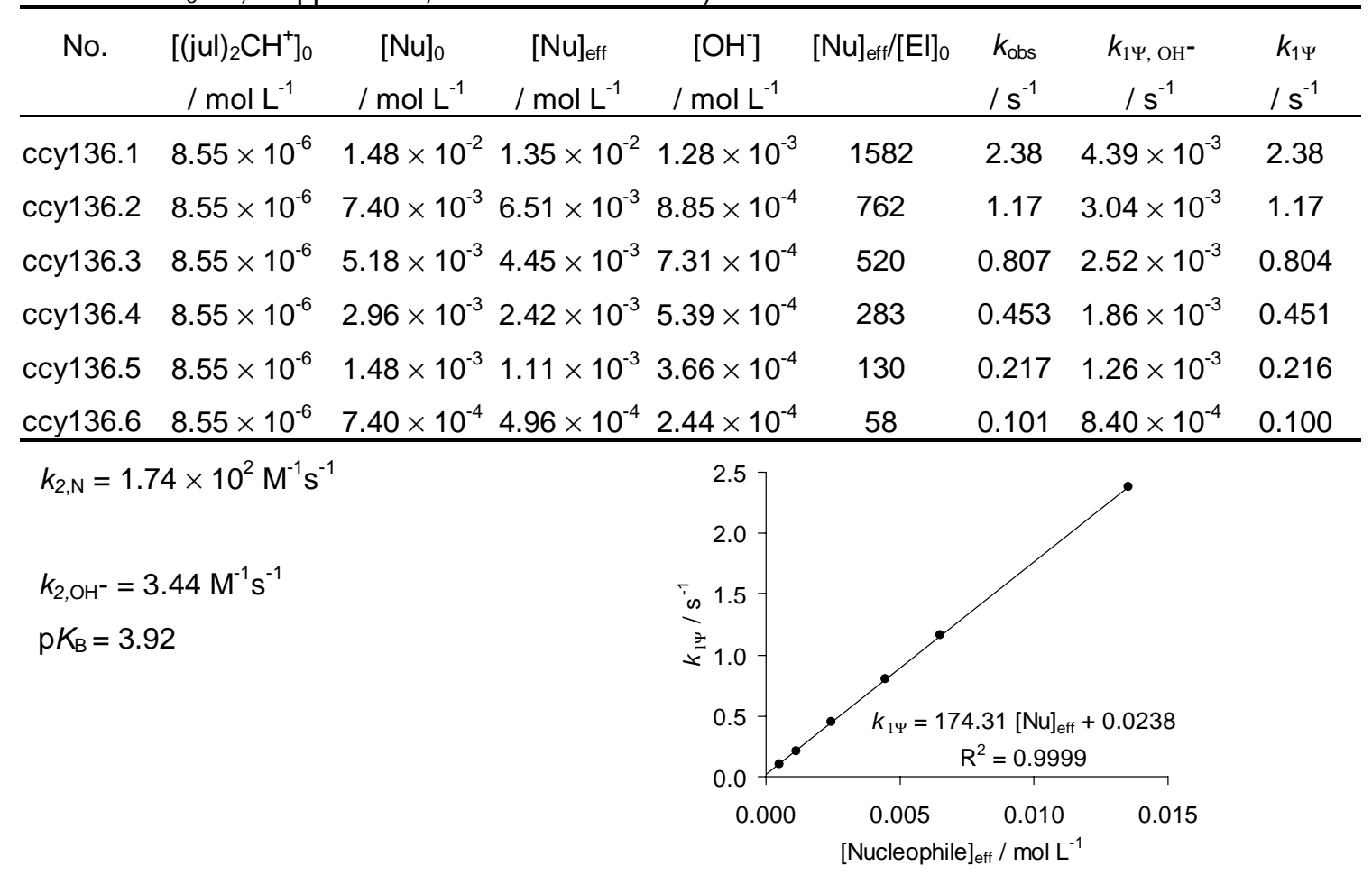


12.1.7. Reaction of 1,2-Ethanediamine (1j) with (lil) $)_{2} \mathrm{CH}^{+} \mathrm{BF}_{4}{ }_{4}$ : (at $20^{\circ} \mathrm{C}$, cosolvent: 0.2 vol- $\% \mathrm{CH}_{3} \mathrm{CN}$, stopped-flow, detection at $634 \mathrm{~nm}$ )

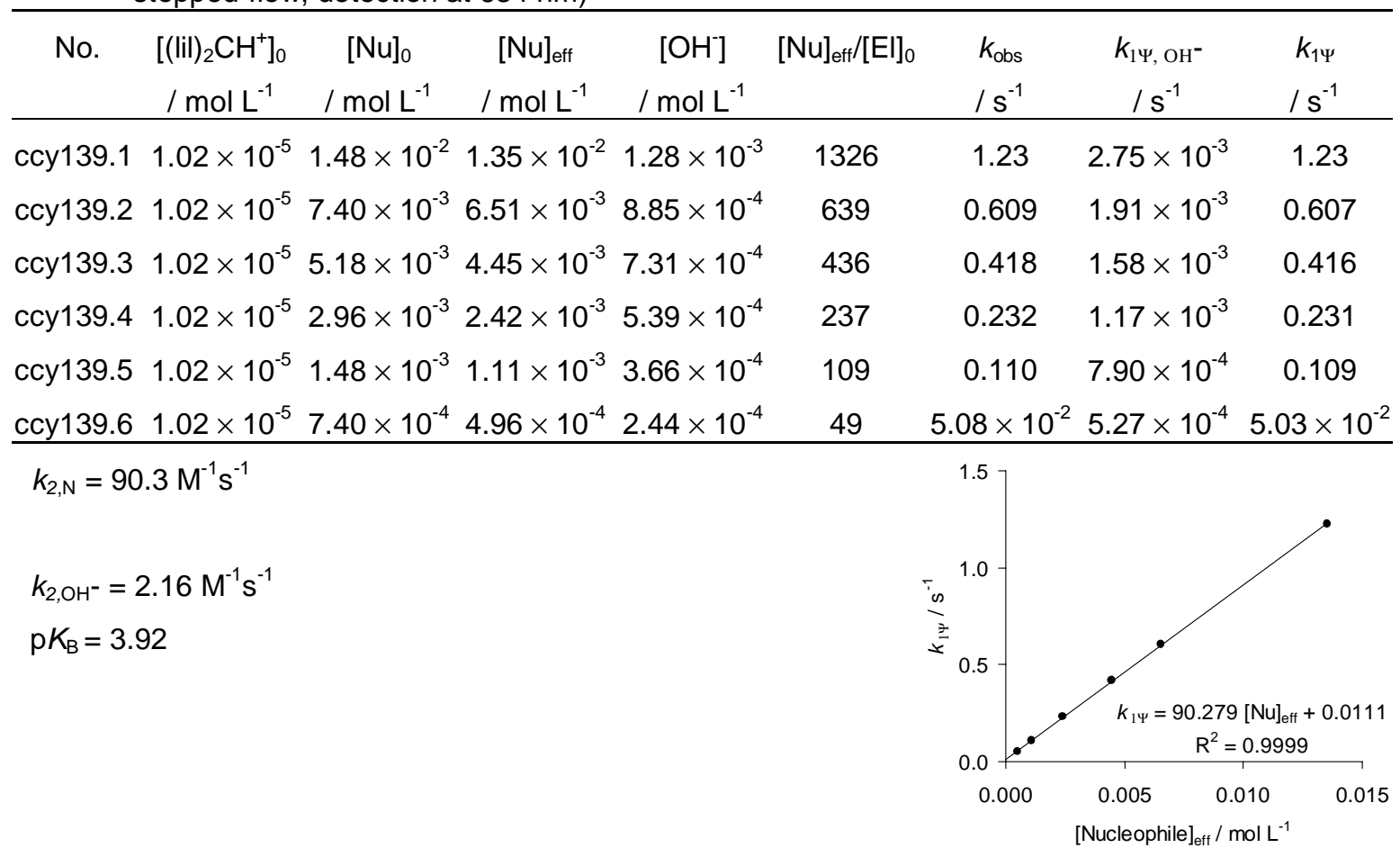

12.2. Reactivity parameters in water: $N=13.28 ; s=0.58$

\begin{tabular}{ccc}
\hline Reference electrophile & $E$ parameter & $k_{2, \mathrm{~N}}\left(20^{\circ} \mathrm{C}\right) / \mathrm{M}^{-1} \mathrm{~s}^{-1}$ \\
\hline (mor $)_{2} \mathrm{CH}^{+}$ & -5.53 & $3.78 \times 10^{4}$ \\
$(\mathrm{dma})_{2} \mathrm{CH}^{+}$ & -7.02 & $4.19 \times 10^{3}$ \\
$(\text { pyr })_{2} \mathrm{CH}^{+}$ & -7.69 & $1.69 \times 10^{3}$ \\
$(\text { thq })_{2} \mathrm{CH}^{+}$ & -8.22 & $8.67 \times 10^{2}$ \\
$(\text { ind })_{2} \mathrm{CH}^{+}$ & -8.76 & $3.78 \times 10^{2}$ \\
$(\mathrm{jul})_{2} \mathrm{CH}^{+}$ & -9.45 & $1.74 \times 10^{2}$ \\
$(\text { lil })_{2} \mathrm{CH}^{+}$ & -10.04 & 90.3 \\
\hline
\end{tabular}

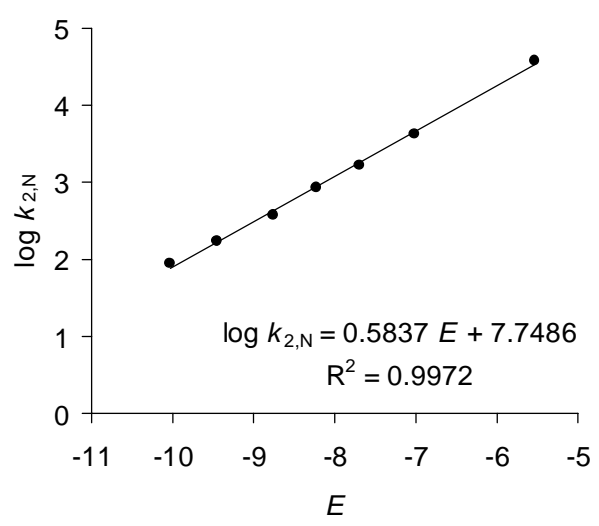




\section{1,3-Propanediamine (1k)}

13.1. Rate constants in water

13.1.1. Reaction of 1,3-Propanediamine (1k) with $(\mathrm{dma})_{2} \mathrm{CH}^{+} \mathrm{BF}_{4}$ : (at $20^{\circ} \mathrm{C}$, cosolvent: 0.5 vol-\% $\mathrm{CH}_{3} \mathrm{CN}$, stopped-flow, detection at $610 \mathrm{~nm}$ )

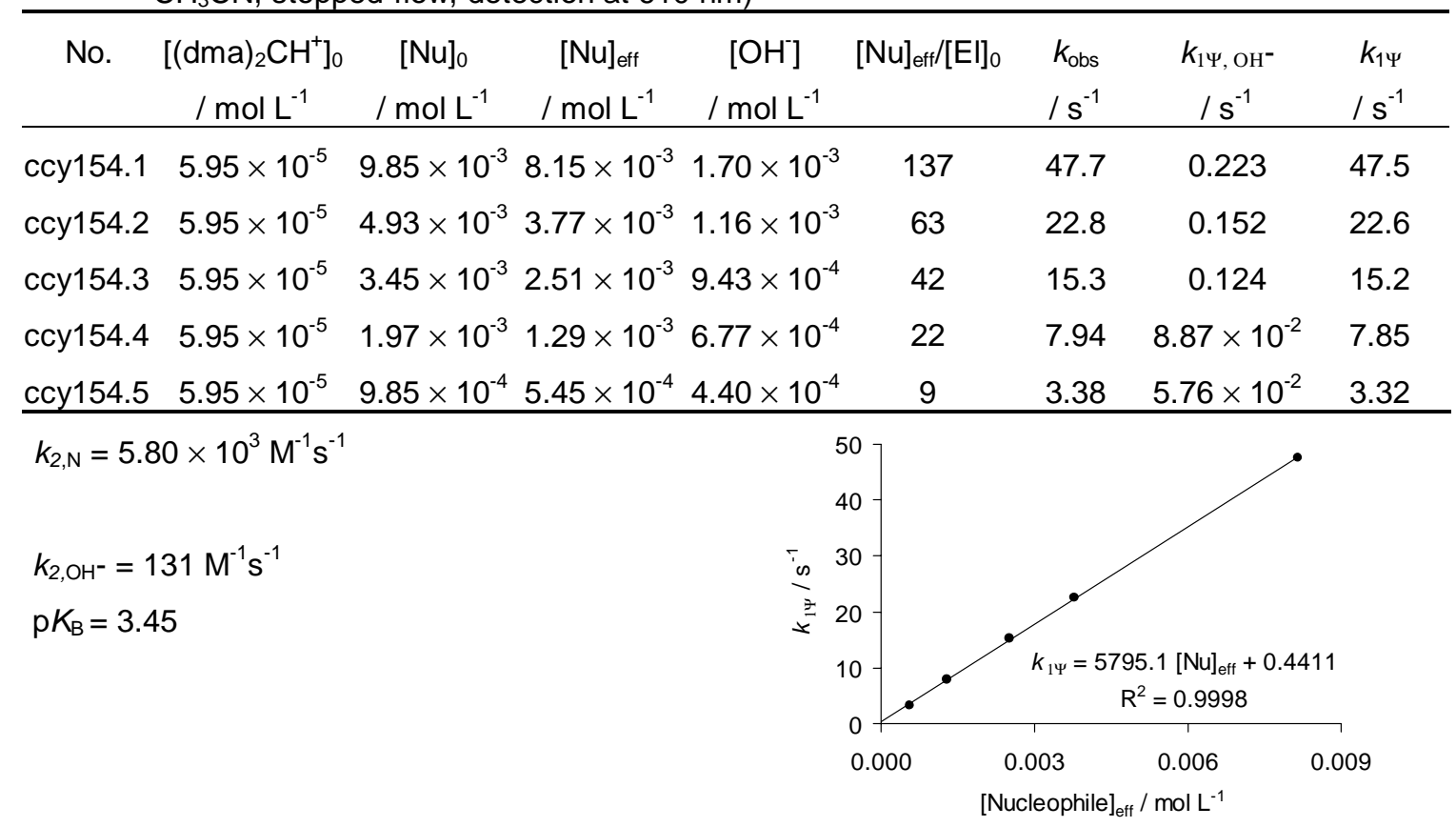

13.1.2. Reaction of 1,3-Propanediamine (1k) with $(\mathrm{pyr})_{2} \mathrm{CH}^{+} \mathrm{BF}_{4}$ : (at $20^{\circ} \mathrm{C}$, cosolvent: 0.1 vol-\% $\mathrm{CH}_{3} \mathrm{CN}$, stopped-flow, detection at $610 \mathrm{~nm}$ )

\begin{tabular}{ccccccccc}
\hline No. & $\begin{array}{c}{\left[(\mathrm{pyr})_{2} \mathrm{CH}^{+}\right]_{0}} \\
/ \mathrm{mol} \mathrm{L}^{-1}\end{array}$ & $\begin{array}{c}{[\mathrm{Nu}]_{0}} \\
/ \mathrm{mol} \mathrm{L}^{-1}\end{array}$ & $\begin{array}{c}{ }^{2} / \mathrm{mol} \mathrm{L}_{\mathrm{eff}}^{-1} \\
{[\mathrm{OH}]}\end{array}$ & $\begin{array}{c}\mathrm{mol} \mathrm{L}^{-1} \\
{[\mathrm{Nu}]_{\mathrm{eff}} /[\mathrm{El}]_{0}}\end{array}$ & $\begin{array}{c}k_{\mathrm{obs}} \\
/ \mathrm{s}^{-1}\end{array}$ & $\begin{array}{c}k_{1 \Psi, \mathrm{OH}^{-}} \\
/ \mathrm{s}^{-1}\end{array}$ & $\begin{array}{c}k_{1 \Psi} \\
/ \mathrm{s}^{-1}\end{array}$ \\
\hline ccy155.1 & $1.13 \times 10^{-5}$ & $9.85 \times 10^{-3}$ & $8.15 \times 10^{-3}$ & $1.70 \times 10^{-3}$ & 721 & 18.8 & $8.25 \times 10^{-2}$ & 18.7 \\
ccy155.2 & $1.13 \times 10^{-5}$ & $4.93 \times 10^{-3}$ & $3.77 \times 10^{-3}$ & $1.16 \times 10^{-3}$ & 334 & 8.90 & $5.61 \times 10^{-2}$ & 8.84 \\
ccy155.3 & $1.13 \times 10^{-5}$ & $3.45 \times 10^{-3}$ & $2.51 \times 10^{-3}$ & $9.43 \times 10^{-4}$ & 222 & 5.95 & $4.57 \times 10^{-2}$ & 5.90 \\
ccy155.4 & $1.13 \times 10^{-5}$ & $1.97 \times 10^{-3}$ & $1.29 \times 10^{-3}$ & $6.77 \times 10^{-4}$ & 114 & 3.12 & $3.28 \times 10^{-2}$ & 3.09 \\
ccy155.5 & $1.13 \times 10^{-5}$ & $9.85 \times 10^{-4}$ & $5.45 \times 10^{-4}$ & $4.40 \times 10^{-4}$ & 48 & 1.38 & $2.13 \times 10^{-2}$ & 1.36 \\
\hline
\end{tabular}

$k_{2, \mathrm{~N}}=2.28 \times 10^{3} \mathrm{M}^{-1} \mathrm{~s}^{-1}$

$k_{2, \mathrm{OH}^{-}}=48.5 \mathrm{M}^{-1} \mathrm{~s}^{-1}$

$\mathrm{p} K_{\mathrm{B}}=3.45$

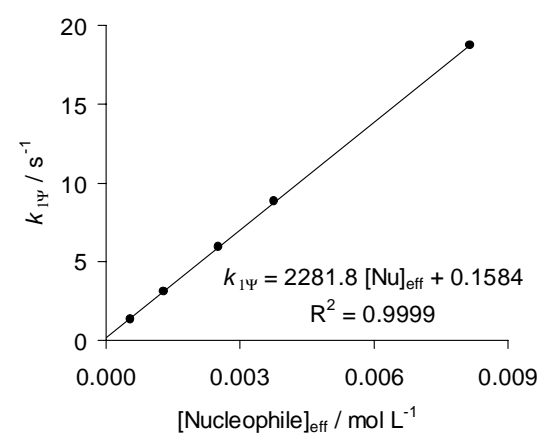


13.1.3. Reaction of 1,3-Propanediamine (1k) with (thq) ${ }_{2} \mathrm{CH}^{+} \mathrm{BF}_{4}^{-}$: (at $20^{\circ} \mathrm{C}$, cosolvent: 0.4 vol-\% $\mathrm{CH}_{3} \mathrm{CN}$, stopped-flow, detection at $610 \mathrm{~nm}$ )

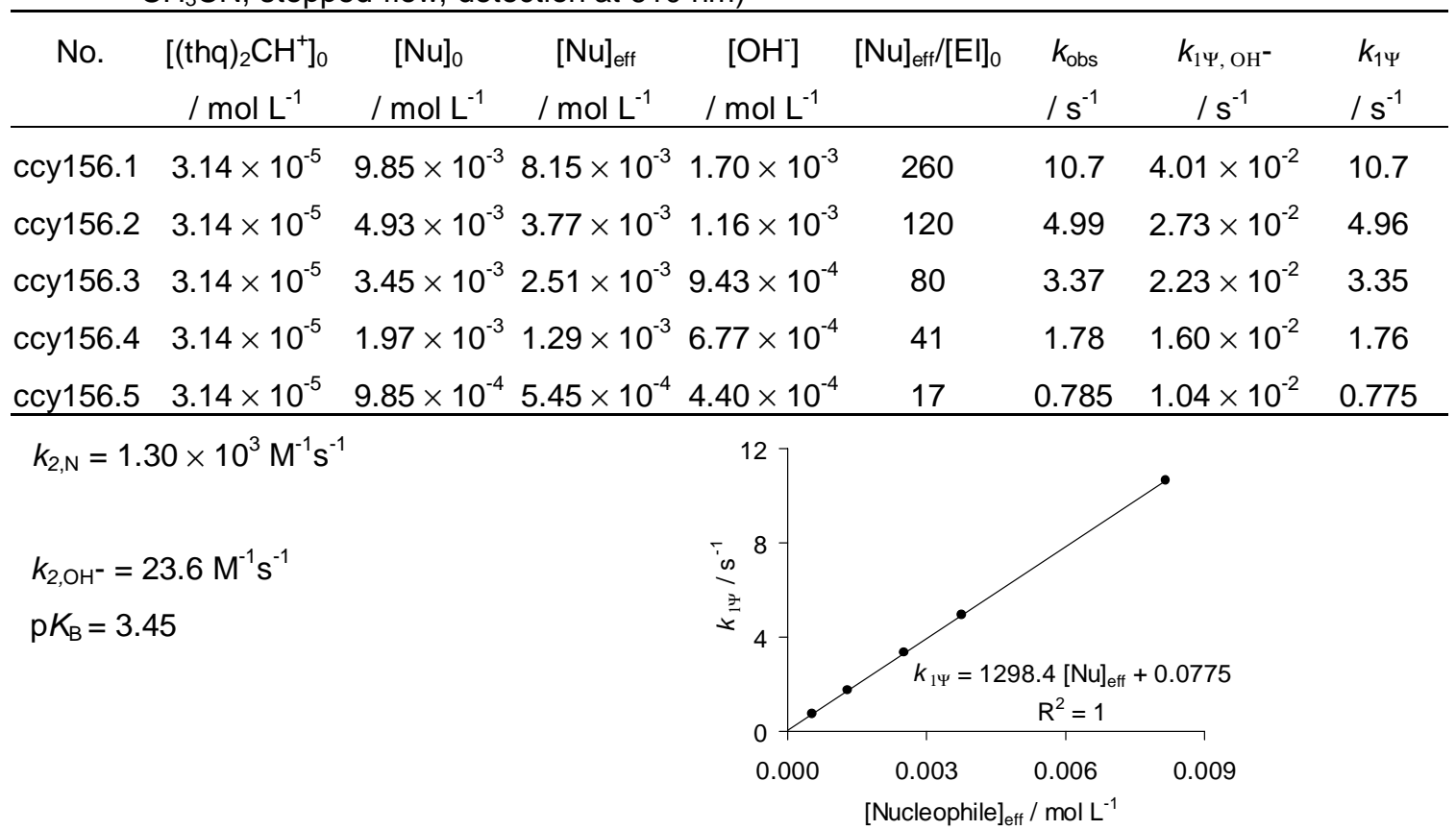

13.1.4. Reaction of 1,3-Propanediamine (1k) with (jul) ${ }_{2} \mathrm{CH}^{+} \mathrm{BF}_{4}{ }_{4}$ : (at $20^{\circ} \mathrm{C}$, cosolvent: 0.1 vol-\% $\mathrm{CH}_{3} \mathrm{CN}$, stopped-flow, detection at $634 \mathrm{~nm}$ )

\begin{tabular}{|c|c|c|c|c|c|c|c|c|}
\hline No. & $\begin{array}{c}{\left[(\mathrm{jul})_{2} \mathrm{CH}^{+}\right]_{0}} \\
/ \mathrm{mol} \mathrm{L}^{-1}\end{array}$ & $\begin{array}{c}{[\mathrm{Nu}]_{0}} \\
/ \mathrm{mol} \mathrm{L}^{-1}\end{array}$ & $\begin{array}{c}{[\mathrm{Nu}]_{\mathrm{eff}}} \\
/ \mathrm{mol} \mathrm{L}^{-1}\end{array}$ & $\begin{array}{c}{\left[\mathrm{OH}^{-}\right]} \\
/ \mathrm{mol} \mathrm{L}^{-1}\end{array}$ & ]o & $\begin{array}{l}k_{\mathrm{obs}} \\
/ \mathrm{s}^{-1}\end{array}$ & $\begin{array}{c}k_{1 \Psi, \mathrm{OH}^{-}} \\
/ \mathrm{s}^{-1} \\
\end{array}$ & $\begin{array}{l}k_{1 \Psi} \\
/ \mathrm{s}^{-1} \\
\end{array}$ \\
\hline & 0.03 & $00 \times \pi$ & 0.10 & $1.10 \times$ & $5 ?$ & 2. & $5.85>$ & 2.24 \\
\hline 7. & $8.55 \times 10^{-6}$ & $4.93 \times 10^{-3}$ & $3.77 \times 10^{-3}$ & $1.16 \times 10$ & 441 & 1.00 & $3.98 \times$ & 1.5 \\
\hline cy 157.3 & $8.55 \times 10^{-6}$ & $3.45 \times 10^{-3}$ & $2.51 \times 10^{-3}$ & $9.43 \times 1$ & 29 & & & 0.712 \\
\hline . & $8.55 \times$ & 1.97 & & 6.77 & & & -3 & 0.375 \\
\hline cy157.5 & $.55 \times 10^{-6}$ & $9.85 \times 10^{-4}$ & $5.45 \times 10^{-4}$ & $4.40 \times 10^{-4}$ & 64 & 0.166 & $1.51 \times 10^{-3}$ & 0.164 \\
\hline
\end{tabular}

$$
\begin{aligned}
& k_{2, \mathrm{~N}}=2.73 \times 10^{2} \mathrm{M}^{-1} \mathrm{~s}^{-1} \\
& k_{2, \mathrm{OH}^{-}}=3.44 \mathrm{M}^{-1} \mathrm{~s}^{-1} \\
& \mathrm{p} K_{\mathrm{B}}=3.45
\end{aligned}
$$

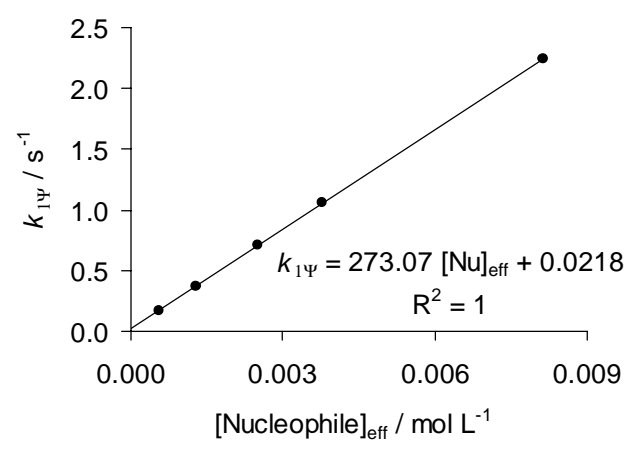


13.1.5. Reaction of 1,3-Propanediamine (1k) with (lil) ${ }_{2} \mathrm{CH}^{+} \mathrm{BF}_{4}{ }_{4}$ : (at $20{ }^{\circ} \mathrm{C}$, cosolvent: 0.2 vol-\% $\mathrm{CH}_{3} \mathrm{CN}$, stopped-flow, detection at $634 \mathrm{~nm}$ )

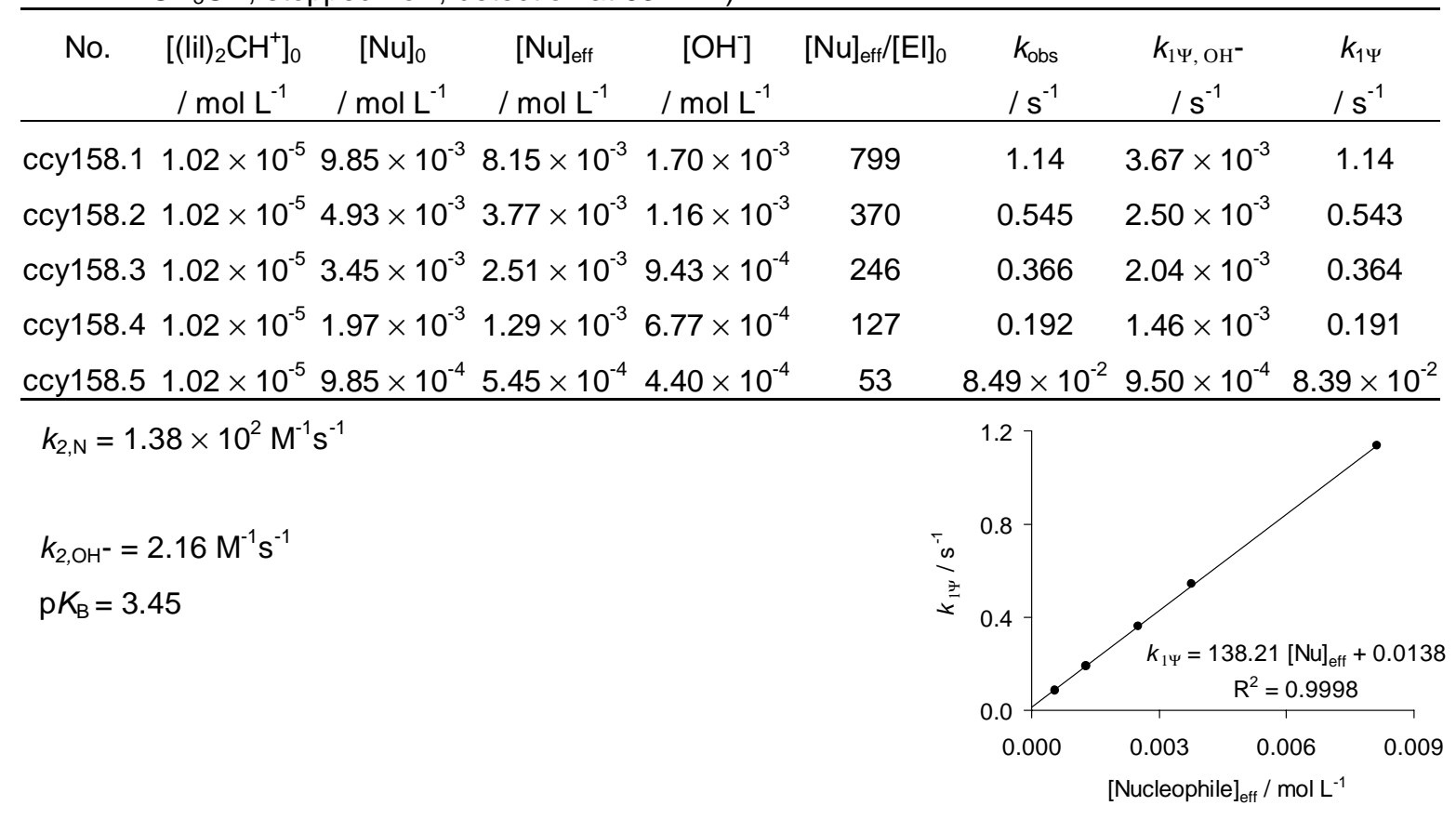

13.2. Reactivity parameters in water: $N=14.02 ; s=0.54$

\begin{tabular}{ccc}
\hline Reference electrophile & $E$ parameter & $k_{2, \mathrm{~N}}\left(20^{\circ} \mathrm{C}\right) / \mathrm{M}^{-1} \mathrm{~s}^{-1}$ \\
\hline$(\mathrm{dma})_{2} \mathrm{CH}^{+}$ & -7.02 & $5.80 \times 10^{3}$ \\
$(\mathrm{pyr})_{2} \mathrm{CH}^{+}$ & -7.69 & $2.28 \times 10^{3}$ \\
$(\mathrm{thq})_{2} \mathrm{CH}^{+}$ & -8.22 & $1.30 \times 10^{3}$ \\
$(\mathrm{jul})_{2} \mathrm{CH}^{+}$ & -9.45 & $2.73 \times 10^{2}$ \\
$(\mathrm{lil})_{2} \mathrm{CH}^{+}$ & -10.04 & $1.38 \times 10^{2}$ \\
\hline
\end{tabular}

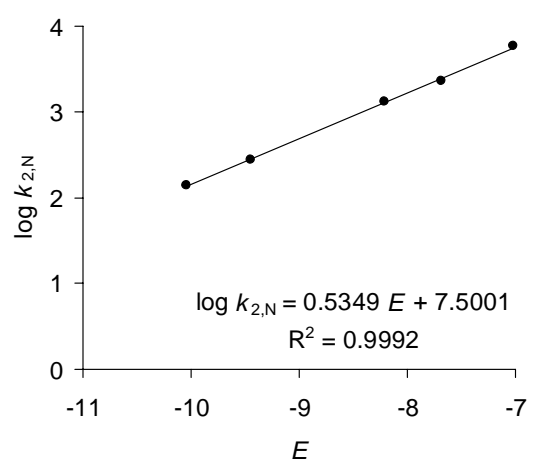




\section{Propargylamine (1I)}

14.1. Rate constants in water

14.1.1. Reaction of Propargylamine (1I) with (mor) ${ }_{2} \mathrm{CH}^{+} \mathrm{BF}_{4}{ }^{-}$(at $20^{\circ} \mathrm{C}$, cosolvent: 9 vol- $\% \mathrm{CH}_{3} \mathrm{CN}$, stopped-flow, detection at $614 \mathrm{~nm}$ )

\begin{tabular}{|c|c|c|c|c|c|c|c|c|}
\hline No. & $\begin{array}{c}{\left[(\mathrm{mor})_{2} \mathrm{CH}^{+}\right]_{0}} \\
/ \mathrm{mol} \mathrm{L}^{-1}\end{array}$ & $\begin{array}{c}{[\mathrm{Nu}]_{0}} \\
/ \mathrm{mol} \mathrm{L}^{-1}\end{array}$ & $\begin{array}{c}{[\mathrm{Nu}]_{\mathrm{eff}}} \\
/ \mathrm{mol} \mathrm{L}^{-1}\end{array}$ & $\begin{array}{c}{\left[\mathrm{OH}^{-}\right]} \\
/ \mathrm{mol} \mathrm{L}^{-1}\end{array}$ & {$[\mathrm{Nu}]_{\mathrm{efff}} /[\mathrm{El}]_{0}$} & $\begin{array}{l}k_{\mathrm{obs}} \\
/ \mathrm{s}^{-1}\end{array}$ & $\begin{array}{c}k_{1 \Psi, \mathrm{OH}^{-}} \\
/ \mathrm{s}^{-1}\end{array}$ & $\begin{array}{l}k_{1 \Psi} \\
/ \mathrm{s}^{-1}\end{array}$ \\
\hline fb193.1 & $2.35 \times 10^{-5}$ & $4.47 \times 10^{-3}$ & $4.39 \times 10^{-3}$ & $7.88 \times 10^{-5}$ & 187 & 52.1 & $8.35 \times 10^{-2}$ & 52.0 \\
\hline $\mathrm{fb} 193.2$ & $2.35 \times 10^{-5}$ & $3.13 \times 10^{-3}$ & $3.06 \times 10^{-3}$ & $6.58 \times 10^{-5}$ & 130 & 36.5 & $6.97 \times 10^{-2}$ & 36.4 \\
\hline $\mathrm{fb} 193.3$ & $2.35 \times 10^{-5}$ & $1.79 \times 10^{-3}$ & $1.74 \times 10^{-3}$ & $4.96 \times 10^{-5}$ & 74 & 21.2 & $5.26 \times 10^{-2}$ & 21.1 \\
\hline fb193.4 & $2.35 \times 10^{-5}$ & $8.94 \times 10^{-4}$ & $8.59 \times 10^{-4}$ & $3.48 \times 10^{-5}$ & 37 & 10.7 & $3.69 \times 10^{-2}$ & 10.7 \\
\hline $\mathrm{fb} 193.5$ & $2.35 \times 10^{-5}$ & $4.47 \times 10^{-4}$ & $4.23 \times 10^{-4}$ & $2.44 \times 10^{-5}$ & 18 & 5.59 & $2.59 \times 10^{-2}$ & 5.56 \\
\hline
\end{tabular}

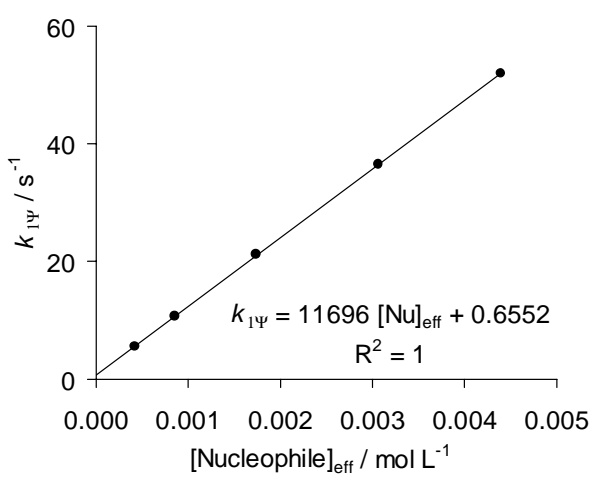

14.1.2. Reaction of Propargylamine (1I) with (dma) ${ }_{2} \mathrm{CH}^{+} \mathrm{BF}_{4}^{-}$(at $20^{\circ} \mathrm{C}$, cosolvent: 0.5 vol- $\% \mathrm{CH}_{3} \mathrm{CN}$, stopped-flow, detection at $610 \mathrm{~nm}$ )

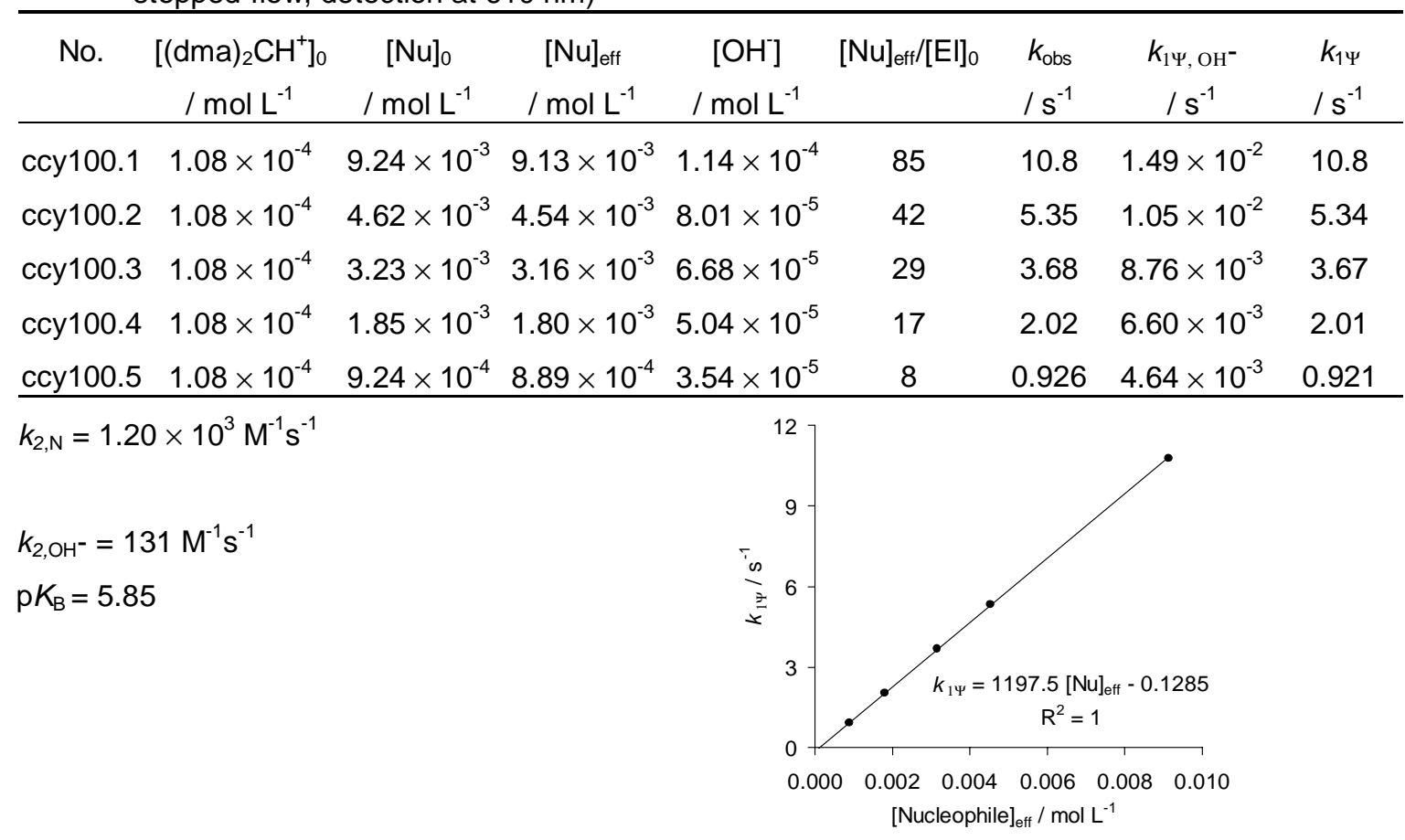


14.1.3. Reaction of Propargylamine (1I) with (pyr) ${ }_{2} \mathrm{CH}^{+} \mathrm{BF}_{4}^{-}$(at $20^{\circ} \mathrm{C}$, cosolvent: 0.2 vol- $\% \mathrm{CH}_{3} \mathrm{CN}$, stopped-flow, detection at $610 \mathrm{~nm}$ )

\begin{tabular}{|c|c|c|c|c|c|c|c|c|}
\hline No. & $\begin{array}{c}{\left[(\mathrm{pyr})_{2} \mathrm{CH}^{+}\right]_{0}} \\
/ \mathrm{mol} \mathrm{L}^{-1}\end{array}$ & $\begin{array}{c}{[\mathrm{Nu}]_{0}} \\
/ \mathrm{mol} \mathrm{L}^{-1}\end{array}$ & $\begin{array}{c}{[\mathrm{Nu}]_{\mathrm{eff}}} \\
/ \mathrm{mol} \mathrm{L}^{-1}\end{array}$ & $\begin{array}{c}{\left[\mathrm{OH}^{-}\right]} \\
/ \mathrm{mol} \mathrm{L}^{-1}\end{array}$ & {$[\mathrm{Nu}$} & $\begin{array}{l}k_{\mathrm{obs}} \\
/ \mathrm{s}^{-1}\end{array}$ & $\begin{array}{c}k_{1 \Psi, \mathrm{OH}^{-}} \\
/ \mathrm{s}^{-1} \\
\end{array}$ & $\begin{array}{l}k_{1 \Psi} \\
/ \mathrm{s}^{-1}\end{array}$ \\
\hline & $1.71 \times 10^{-5}$ & $9.24 \times 10^{-3}$ & $9.13 \times 10^{-3}$ & $1.14 \times 10^{-5}$ & & 409 & $5.51 \times 1$ & 4.08 \\
\hline ccy & $1.71 \times 10^{-5}$ & $4.62 \times 10^{-3}$ & $4.54 \times$ & $8.01 \times 10^{-5}$ & 2 & 2.0 & & \\
\hline 3 & 1.71 & 3.23 & & & & & & 1.44 \\
\hline cy101.4 & $1.71 \times 10^{-5}$ & $1.85 \times 10^{-3}$ & $1.80 \times$ & $5.04 \times 10^{-}$ & 10 & 0.822 & & 0.820 \\
\hline cy101.5 & $1.71 \times 10^{-5}$ & $9.24 \times 10^{-4}$ & $8.89 \times 10^{-4}$ & $3.54 \times 10^{-5}$ & L & 0.406 & $1.72 \times 10^{-3}$ & 0.404 \\
\hline
\end{tabular}

$$
\begin{aligned}
& k_{2, \mathrm{~N}}=4.47 \times 10^{2} \mathrm{M}^{-1} \mathrm{~s}^{-1} \\
& k_{2, \mathrm{OH}}=48.5 \mathrm{M}^{-1} \mathrm{~s}^{-1} \\
& \mathrm{p} K_{\mathrm{B}}=5.85
\end{aligned}
$$

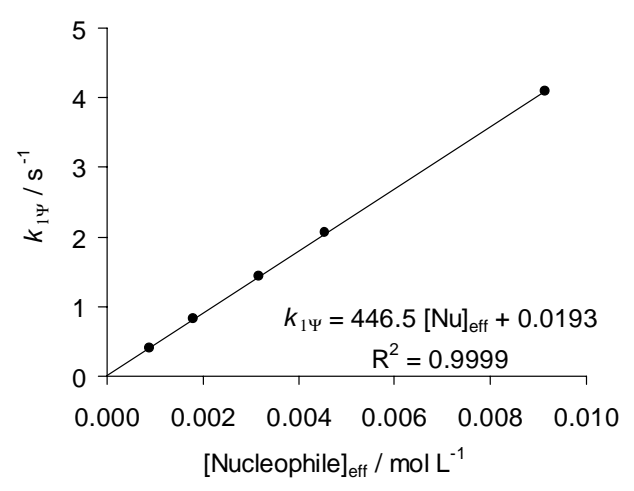

14.1.4. Reaction of Propargylamine (1I) with (thq) ${ }_{2} \mathrm{CH}^{+} \mathrm{BF}_{4}{ }^{-}$(at $20^{\circ} \mathrm{C}$, cosolvent: 0.2 vol- $\% \mathrm{CH}_{3} \mathrm{CN}$, stopped-flow, detection at $610 \mathrm{~nm}$ )

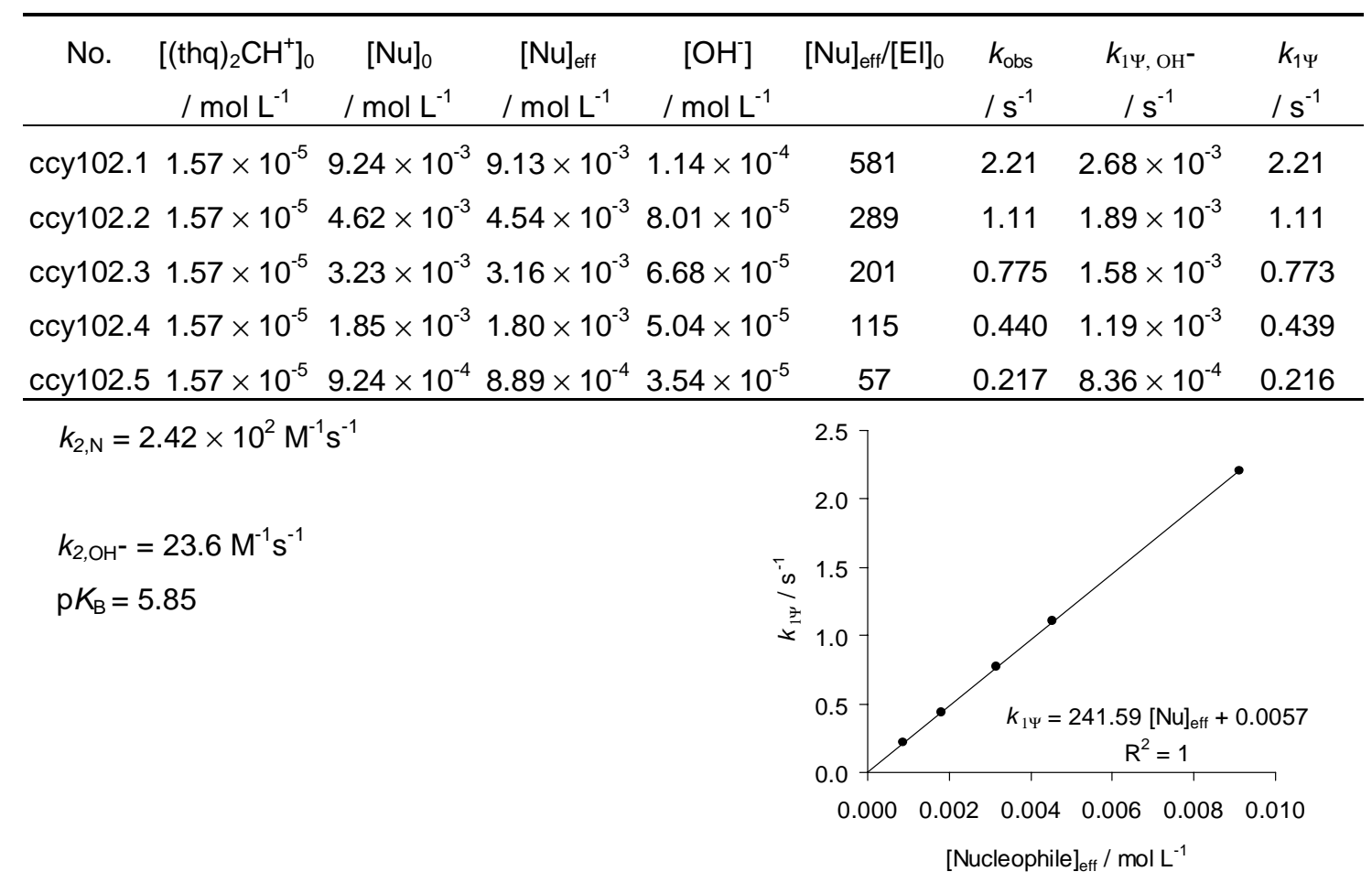


14.1.5. Reaction of Propargylamine (1I) with (ind) ${ }_{2} \mathrm{CH}^{+} \mathrm{BF}_{4}{ }^{-}$(at $20^{\circ} \mathrm{C}$, cosolvent: 0.2 vol- $\% \mathrm{CH}_{3} \mathrm{CN}$, stopped-flow, detection at $614 \mathrm{~nm}$ )

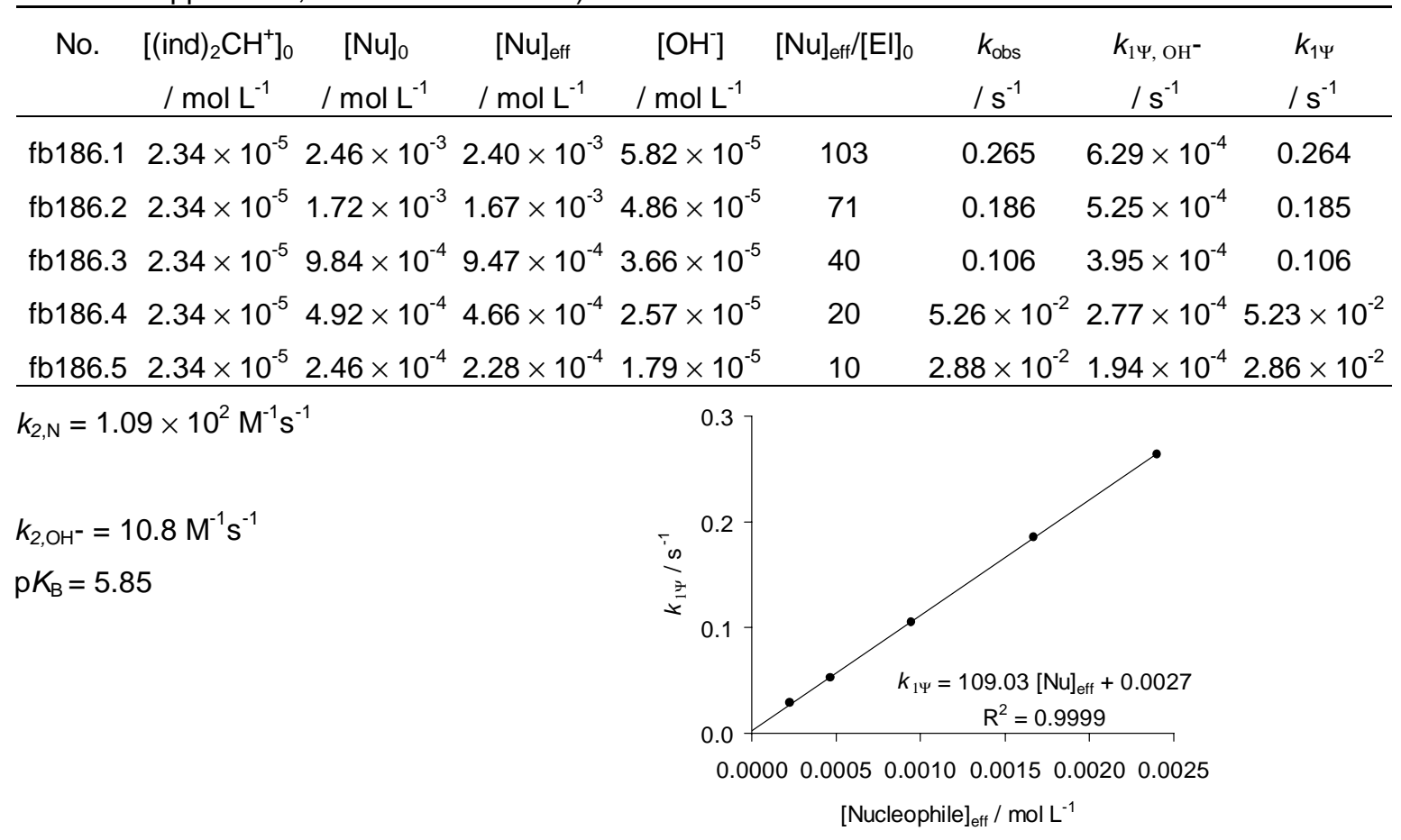

14.1.6. Reaction of Propargylamine (1I) with (jul) ${ }_{2} \mathrm{CH}^{+} \mathrm{BF}_{4}^{-}$(at $20^{\circ} \mathrm{C}$, cosolvent: 0.2 vol- $\% \mathrm{CH}_{3} \mathrm{CN}$, stopped-flow, detection at $635 \mathrm{~nm}$ )

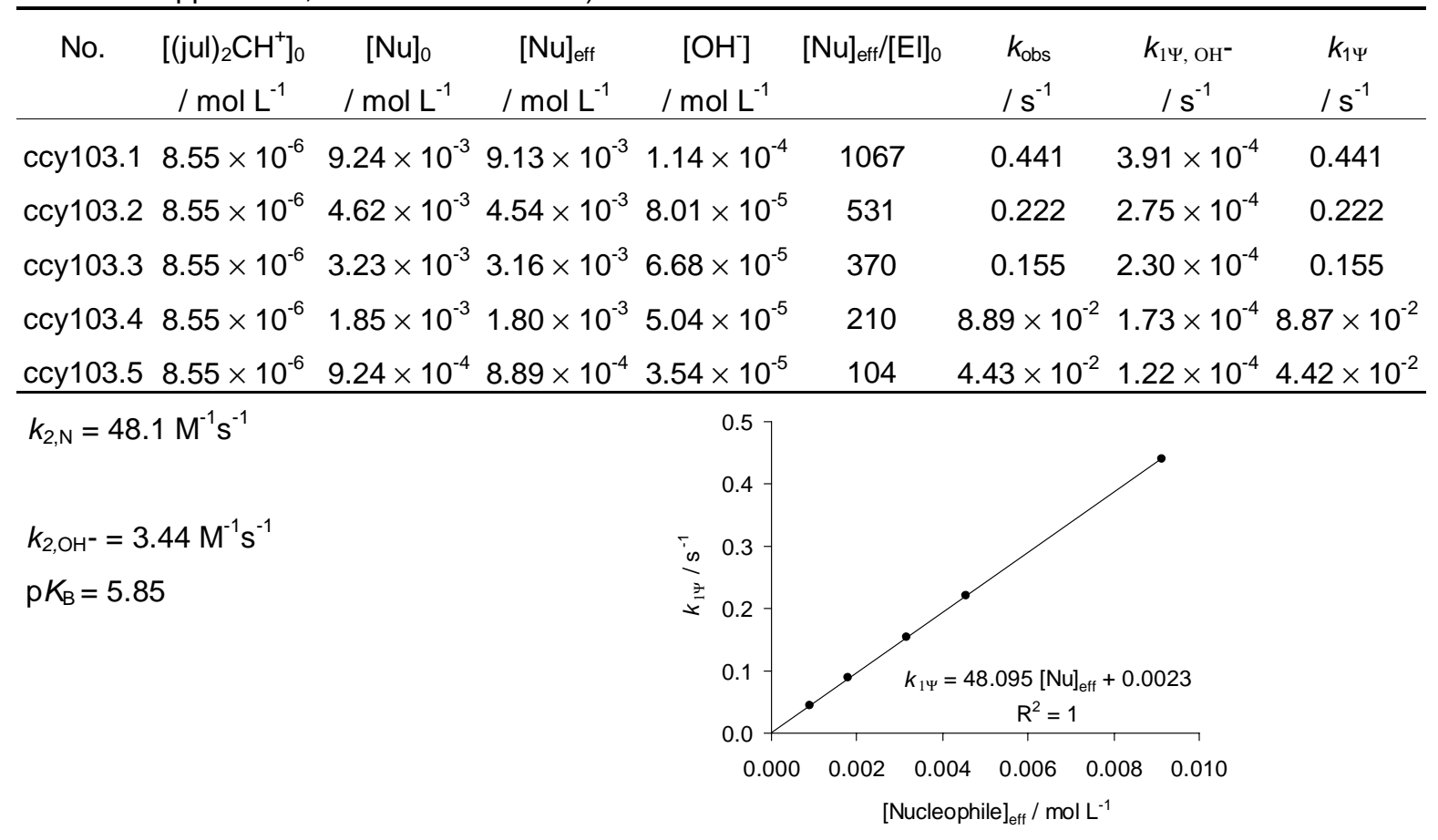


14.1.7. Reaction of Propargylamine (1I) with (lii) ${ }_{2} \mathrm{CH}^{+} \mathrm{BF}_{4}^{-}$(at $20^{\circ} \mathrm{C}$, cosolvent: 0.2 vol- $\% \mathrm{CH}_{3} \mathrm{CN}$, stopped-flow, detection at $635 \mathrm{~nm}$ )

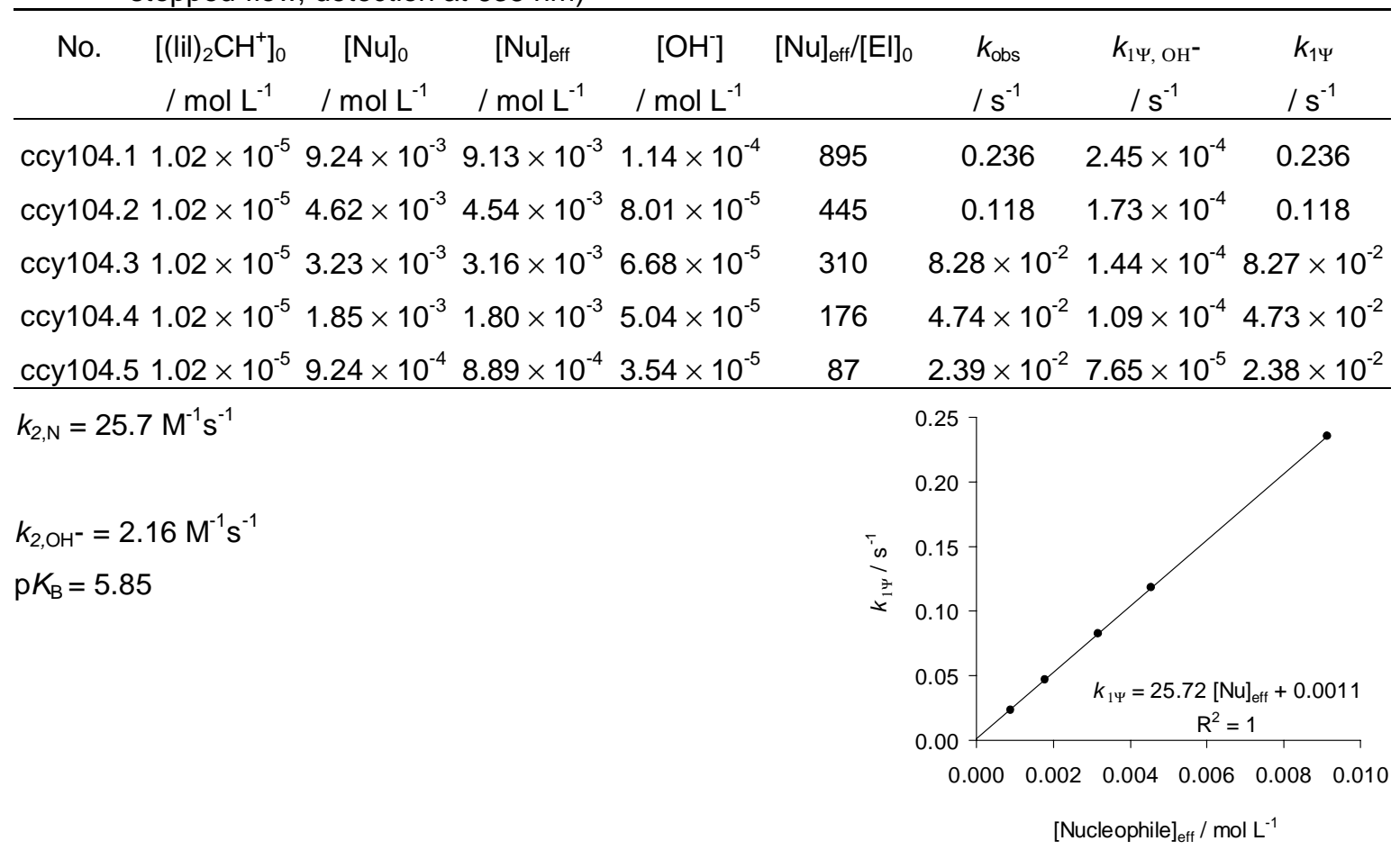

14.2. Reactivity parameters in water: $N=12.29 ; s=0.59$

\begin{tabular}{ccc}
\hline Reference electrophile & $E$ parameter & $k_{2, \mathrm{~N}}\left(20^{\circ} \mathrm{C}\right) / \mathrm{M}^{-1} \mathrm{~s}^{-1}$ \\
\hline$(\mathrm{mor})_{2} \mathrm{CH}^{+}$ & -5.53 & $1.17 \times 10^{4}$ \\
$(\mathrm{dma})_{2} \mathrm{CH}^{+}$ & -7.02 & $1.20 \times 10^{3}$ \\
$(\mathrm{pyr})_{2} \mathrm{CH}^{+}$ & -7.69 & $4.47 \times 10^{2}$ \\
$(\mathrm{thq})_{2} \mathrm{CH}^{+}$ & -8.22 & $2.42 \times 10^{2}$ \\
$(\text { ind })_{2} \mathrm{CH}^{+}$ & -8.76 & $1.09 \times 10^{2}$ \\
$(\mathrm{jul})_{2} \mathrm{CH}^{+}$ & -9.45 & 48.1 \\
$(\mathrm{lil})_{2} \mathrm{CH}^{+}$ & -10.04 & 25.7 \\
\hline
\end{tabular}

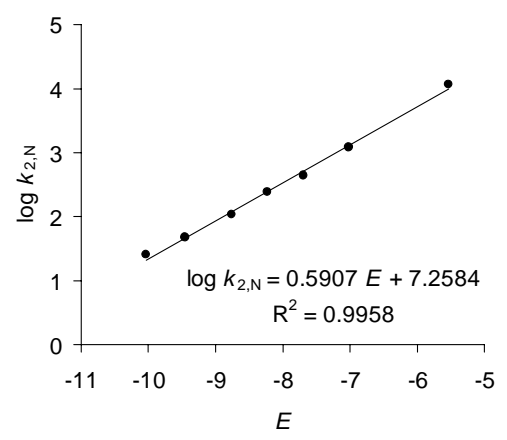




\section{Allylamine (1m)}

15.1. Rate constants in water

15.1.1. Reaction of Allylamine (1m) with $(\mathrm{dma})_{2} \mathrm{CH}^{+} \mathrm{BF}_{4}^{-}$(at $20^{\circ} \mathrm{C}$, cosolvent: 0.5 vol- $\% \mathrm{CH}_{3} \mathrm{CN}$, stopped-flow, detection at $610 \mathrm{~nm}$ )

\begin{tabular}{ccccccccc}
\hline No. & $\begin{array}{c}{\left[(\mathrm{dma})_{2} \mathrm{CH}^{+}\right]_{0}} \\
/ \mathrm{mol} \mathrm{L}^{-1}\end{array}$ & $\begin{array}{c}{[\mathrm{Nu}]_{0}} \\
/ \mathrm{mol} \mathrm{L}^{-1}\end{array}$ & $\begin{array}{c}{[\mathrm{Nu}]_{\mathrm{eff}}} \\
/ \mathrm{mol} \mathrm{L}^{-1}\end{array}$ & $\begin{array}{c}{[\mathrm{OH}]} \\
/ \mathrm{mol} \mathrm{L}^{-1}\end{array}$ & $\begin{array}{c}{[\mathrm{Nu}]_{\mathrm{eff}} /[\mathrm{El}]_{0}} \\
k_{\mathrm{obs}}\end{array}$ & $\begin{array}{c}k_{1 \Psi, \mathrm{OH}^{-}} \\
/ \mathrm{s}^{-1}\end{array}$ & $\begin{array}{c}k_{1 \Psi} \\
/ \mathrm{s}^{-1}\end{array}$ & $/ \mathrm{s}^{-1}$ \\
\hline ccy106.1 & $1.08 \times 10^{-4}$ & $4.08 \times 10^{-3}$ & $3.74 \times 10^{-3}$ & $3.40 \times 10^{-4}$ & 35 & 8.63 & $4.45 \times 10^{-2}$ & 8.59 \\
ccy106.2 & $1.08 \times 10^{-4}$ & $2.04 \times 10^{-3}$ & $1.80 \times 10^{-3}$ & $2.36 \times 10^{-4}$ & 17 & 4.11 & $3.09 \times 10^{-2}$ & 4.08 \\
ccy106.3 & $1.08 \times 10^{-4}$ & $1.43 \times 10^{-3}$ & $1.23 \times 10^{-3}$ & $1.95 \times 10^{-4}$ & 11 & 2.75 & $2.56 \times 10^{-2}$ & 2.72 \\
ccy106.4 & $1.08 \times 10^{-4}$ & $8.16 \times 10^{-4}$ & $6.72 \times 10^{-4}$ & $1.44 \times 10^{-4}$ & 6 & 1.38 & $1.89 \times 10^{-2}$ & 1.36 \\
\hline
\end{tabular}

$k_{2, \mathrm{~N}}=2.35 \times 10^{3} \mathrm{M}^{-1} \mathrm{~s}^{-1}$

$k_{2, \mathrm{OH}^{-}}=131 \mathrm{M}^{-1} \mathrm{~s}^{-1}$

$\mathrm{p} K_{\mathrm{B}}=4.51$

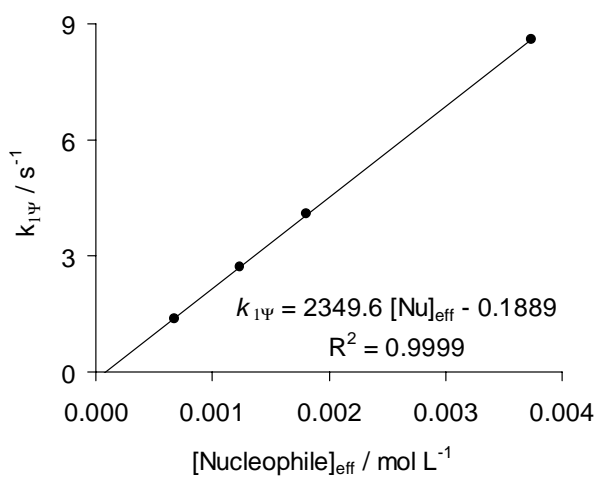

15.1.2. Reaction of Allylamine (1m) with (pyr) $)_{2} \mathrm{CH}^{+} \mathrm{BF}_{4}^{-}$(at $20^{\circ} \mathrm{C}$, cosolvent: 0.2 vol-\% $\mathrm{CH}_{3} \mathrm{CN}$, stopped-flow, detection at $610 \mathrm{~nm}$ )

\begin{tabular}{ccccccccc}
\hline No. & $\begin{array}{c}{\left[(\mathrm{pyr})_{2} \mathrm{CH}^{+}\right]_{0}} \\
/ \mathrm{mol} \mathrm{L}^{-1}\end{array}$ & $\begin{array}{c}{[\mathrm{Nu}]_{0}} \\
/ \mathrm{mol} \mathrm{L}^{-1}\end{array}$ & $\begin{array}{c}{[\mathrm{Nu}]_{\mathrm{eff}}} \\
/ \mathrm{mol} \mathrm{L}^{-1}\end{array}$ & $\begin{array}{c}{[\mathrm{OH}]} \\
/ \mathrm{mol} \mathrm{L}^{-1}\end{array}$ & {$[\mathrm{Nu}]_{\mathrm{eff}} /[\mathrm{El}]_{0}$} & $\begin{array}{c}k_{\mathrm{obs}} \\
/ \mathrm{s}^{-1}\end{array}$ & $\begin{array}{c}k_{1 \Psi, \mathrm{OH}^{-}} \\
/ \mathrm{s}^{-1}\end{array}$ & $\begin{array}{c}k_{1 \Psi} \\
/ \mathrm{s}^{-1}\end{array}$ \\
\hline ccy107.1 & $1.71 \times 10^{-5}$ & $4.08 \times 10^{-3}$ & $3.74 \times 10^{-3}$ & $3.40 \times 10^{-4}$ & 219 & 3.58 & $1.65 \times 10^{-2}$ & 3.56 \\
ccy107.2 & $1.71 \times 10^{-5}$ & $2.04 \times 10^{-3}$ & $1.80 \times 10^{-3}$ & $2.36 \times 10^{-4}$ & 105 & 1.78 & $1.15 \times 10^{-2}$ & 1.77 \\
ccy107.3 & $1.71 \times 10^{-5}$ & $1.43 \times 10^{-3}$ & $1.23 \times 10^{-3}$ & $1.95 \times 10^{-4}$ & 72 & 1.20 & $9.47 \times 10^{-3}$ & 1.19 \\
ccy107.4 & $1.71 \times 10^{-5}$ & $8.16 \times 10^{-4}$ & $6.72 \times 10^{-4}$ & $1.44 \times 10^{-4}$ & 39 & 0.648 & $6.99 \times 10^{-3}$ & 0.641 \\
ccy107.5 & $1.71 \times 10^{-5}$ & $4.08 \times 10^{-4}$ & $3.10 \times 10^{-4}$ & $9.79 \times 10^{-5}$ & 18 & 0.291 & $4.75 \times 10^{-3}$ & 0.286 \\
\hline
\end{tabular}

$k_{2, \mathrm{~N}}=9.55 \times 10^{2} \mathrm{M}^{-1} \mathrm{~s}^{-1}$

$k_{2, \mathrm{OH}^{-}}=48.5 \mathrm{M}^{-1} \mathrm{~s}^{-1}$

$\mathrm{p} K_{\mathrm{B}}=4.51$

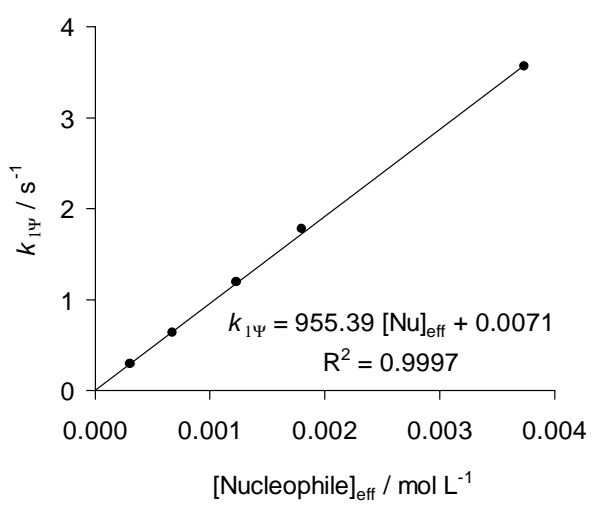


15.1.3. Reaction of Allylamine (1 $\mathbf{m}$ ) with (thq) ${ }_{2} \mathrm{CH}^{+} \mathrm{BF}_{4}{ }^{-}$(at $20^{\circ} \mathrm{C}$, cosolvent: 0.2 vol- $\% \mathrm{CH}_{3} \mathrm{CN}$, stopped-flow, detection at $610 \mathrm{~nm}$ )

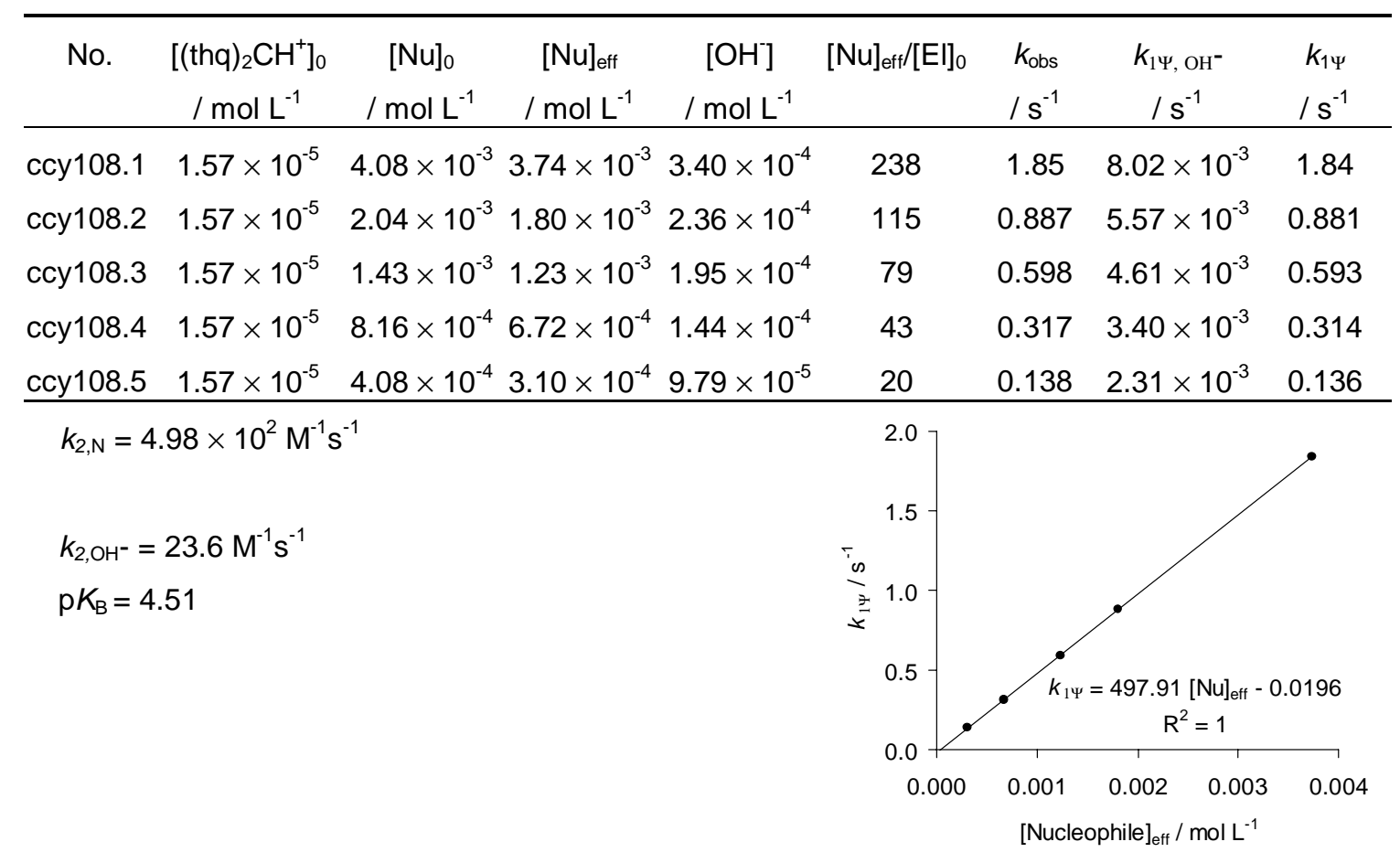

15.1.4. Reaction of Allylamine (1m) with $(\mathrm{jul})_{2} \mathrm{CH}^{+} \mathrm{BF}_{4}^{-}$(at $20^{\circ} \mathrm{C}$, cosolvent: 0.2 vol- $\% \mathrm{CH}_{3} \mathrm{CN}$, stopped-flow, detection at $634 \mathrm{~nm}$ )

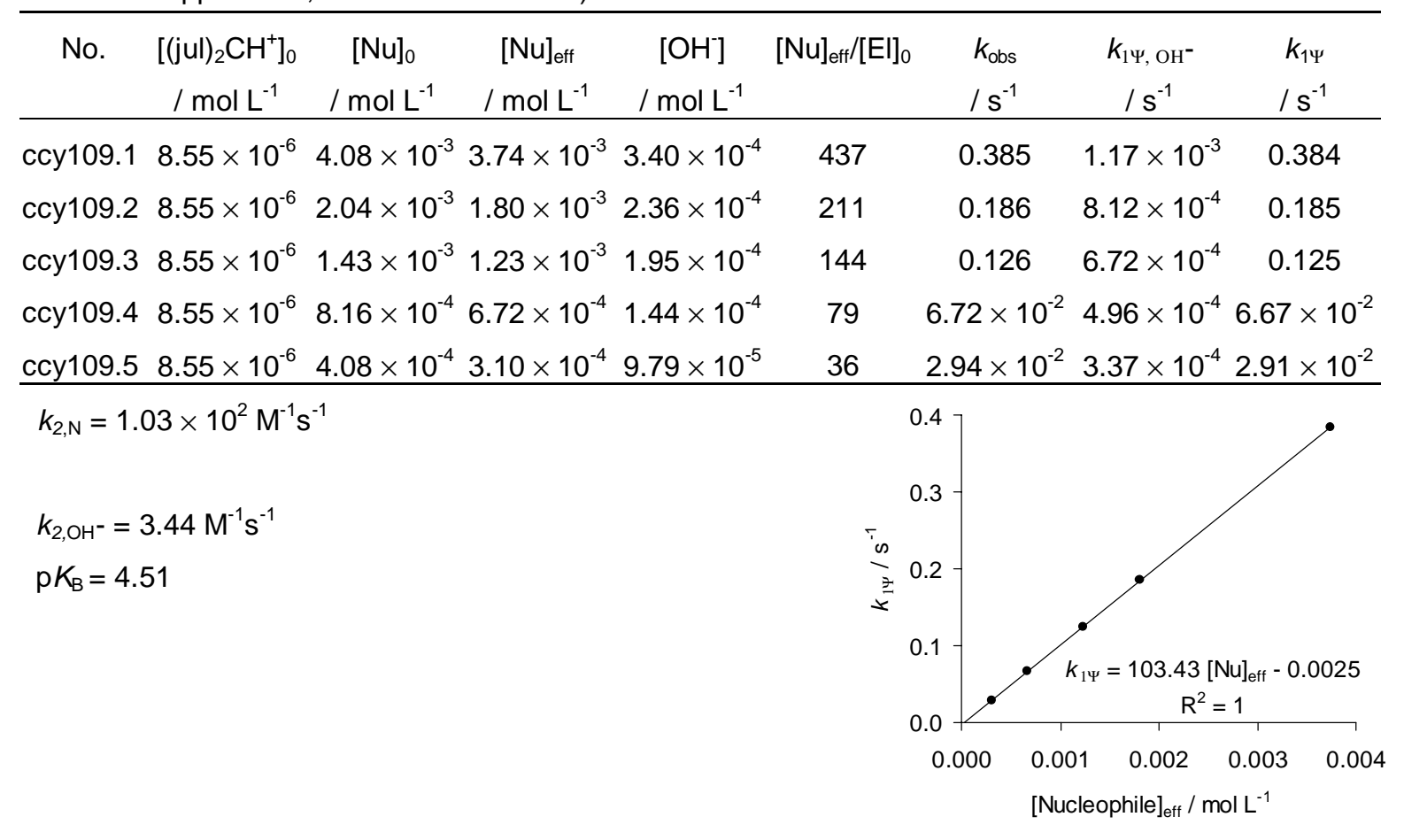


15.1.5. Reaction of Allylamine (1m) with (lil) ${ }_{2} \mathrm{CH}^{+} \mathrm{BF}_{4}{ }_{4}$ (at $20^{\circ} \mathrm{C}$, cosolvent: 0.2 vol-\% $\mathrm{CH}_{3} \mathrm{CN}$, stopped-flow, detection at $634 \mathrm{~nm}$ )

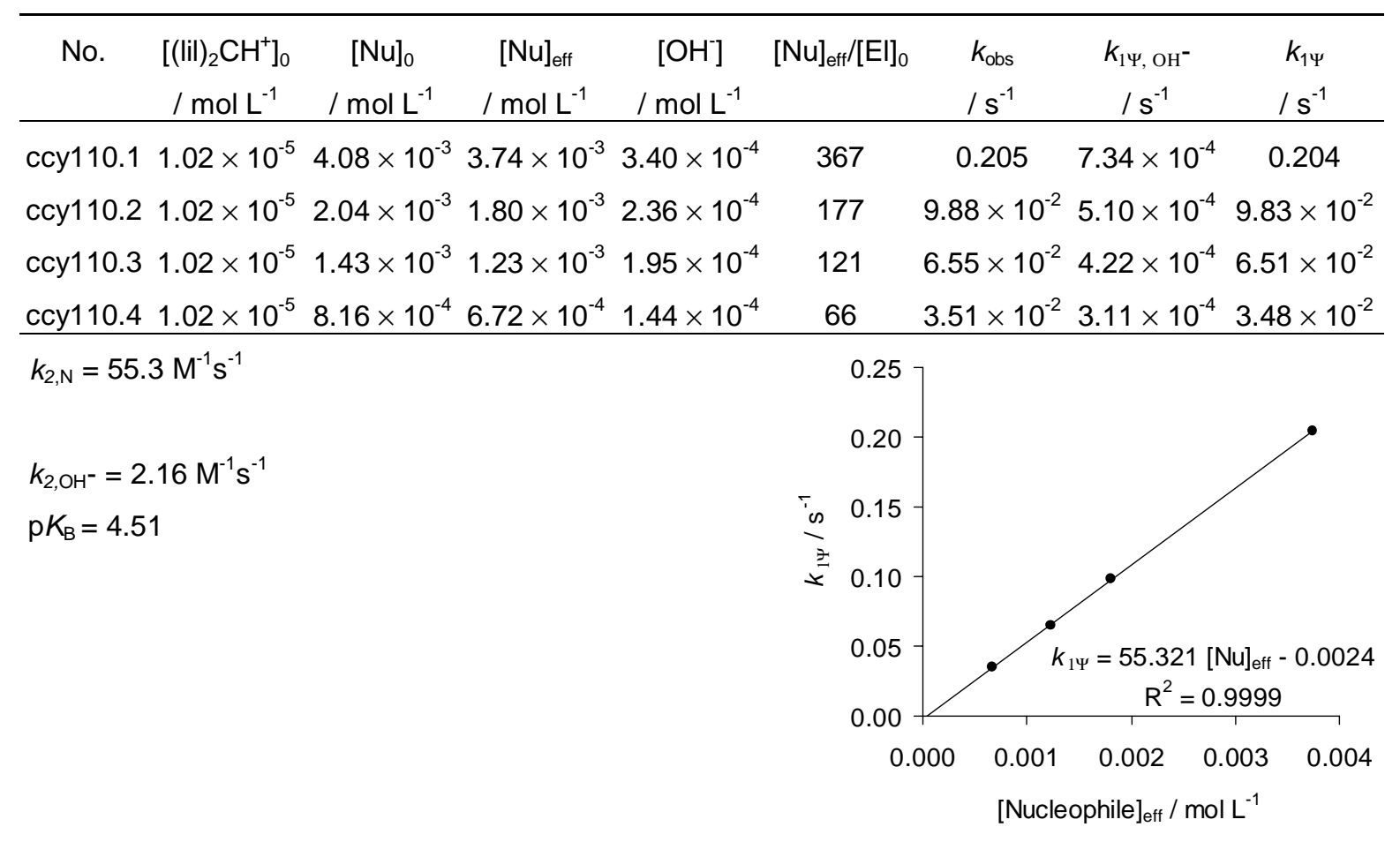

15.2. Reactivity parameters in water: $N=13.21 ; s=0.54$

\begin{tabular}{ccc}
\hline Reference electrophile & $E$ parameter & $k_{2, \mathrm{~N}}\left(20^{\circ} \mathrm{C}\right) / \mathrm{M}^{-1} \mathrm{~s}^{-1}$ \\
\hline$(\mathrm{dma})_{2} \mathrm{CH}^{+}$ & -7.02 & $2.35 \times 10^{3}$ \\
$(\mathrm{pyr})_{2} \mathrm{CH}^{+}$ & -7.69 & $9.55 \times 10^{2}$ \\
$(\text { thq })_{2} \mathrm{CH}^{+}$ & -8.22 & $4.98 \times 10^{2}$ \\
$(\mathrm{jul})_{2} \mathrm{CH}^{+}$ & -9.45 & $1.03 \times 10^{2}$ \\
$(\mathrm{lil})_{2} \mathrm{CH}^{+}$ & -10.04 & 55.3 \\
\hline
\end{tabular}

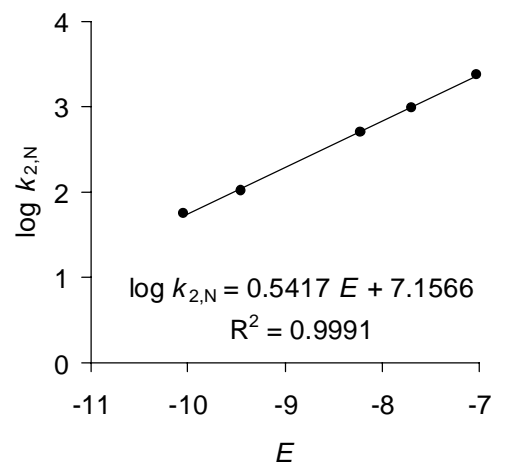




\section{Benzylamine (1n)}

16.1. Rate constants in water

16.1.1. Reaction of Benzylamine (1n) with (mor) ${ }_{2} \mathrm{CH}^{+} \mathrm{BF}_{4}^{-}$(at $20^{\circ} \mathrm{C}$, cosolvent: 9 vol- $\% \mathrm{CH}_{3} \mathrm{CN}$, stopped-flow, detection at $607 \mathrm{~nm}$ )

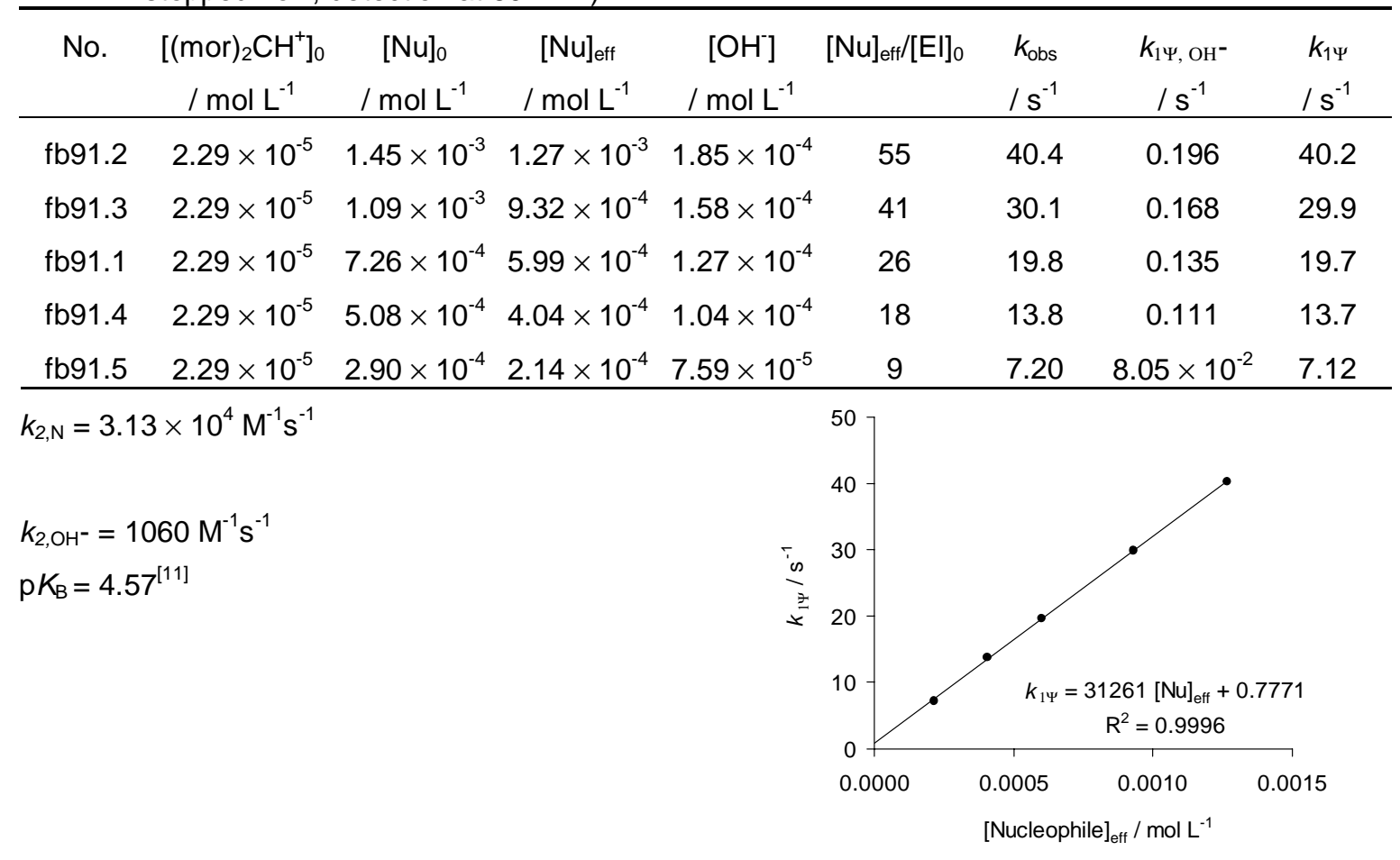

16.1.2. Reaction of Benzylamine (1n) with (dma) ${ }_{2} \mathrm{CH}^{+} \mathrm{BF}_{4}^{-}$(at $20^{\circ} \mathrm{C}$, cosolvent: 0.4 vol-\% $\mathrm{CH}_{3} \mathrm{CN}$, stopped-flow, detection at $607 \mathrm{~nm}$ )

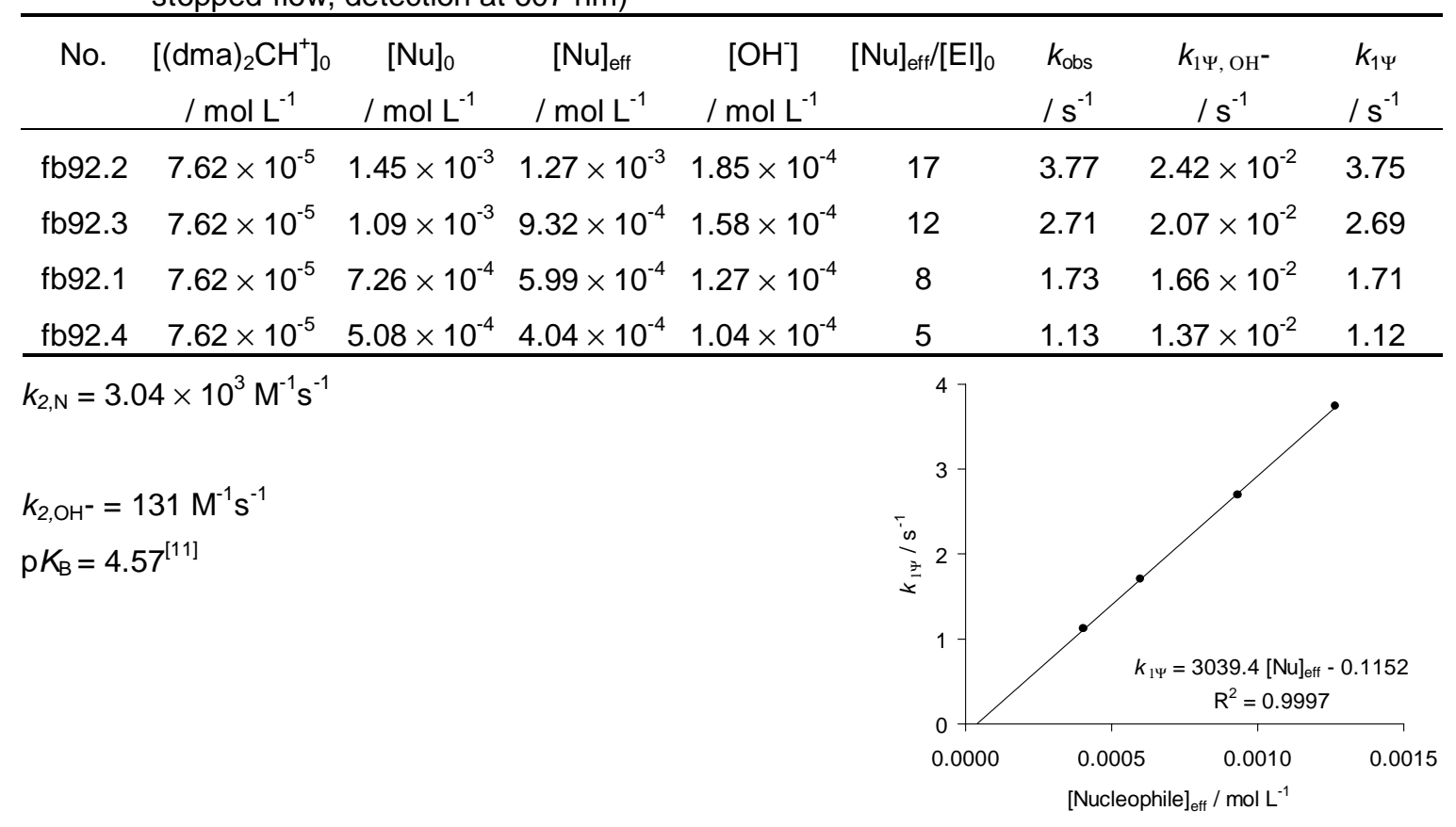


16.1.3. Reaction of Benzylamine (1n) with (pyr) ${ }_{2} \mathrm{CH}^{+} \mathrm{BF}_{4}{ }^{-}$(at $20{ }^{\circ} \mathrm{C}$, cosolvent: 0.2 vol- $\% \mathrm{CH}_{3} \mathrm{CN}$, stopped-flow, detection at $607 \mathrm{~nm}$ )

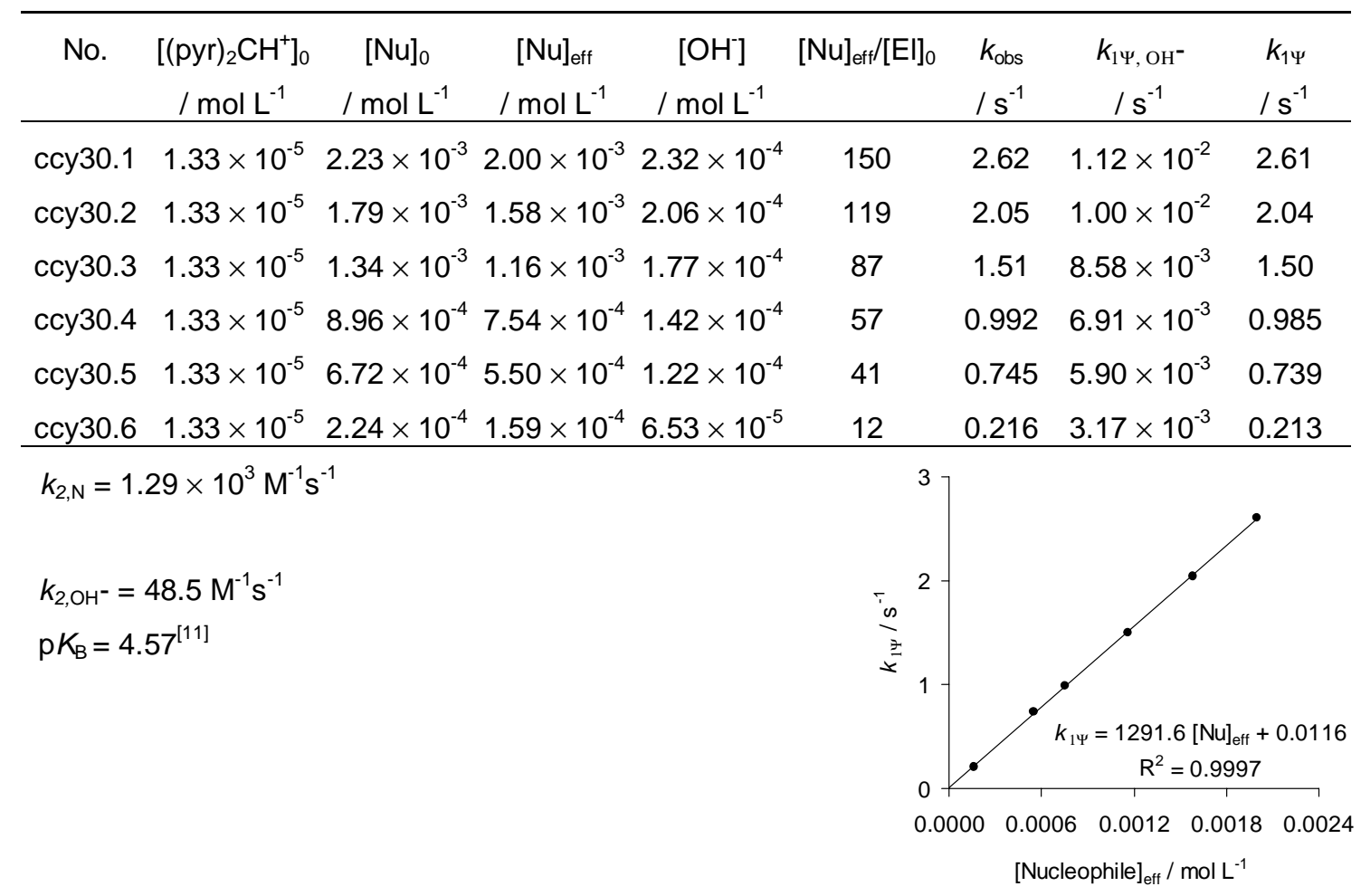

16.1.4. Reaction of Benzylamine (1n) with (thq) ${ }_{2} \mathrm{CH}^{+} \mathrm{BF}_{4}^{-}$(at $20{ }^{\circ} \mathrm{C}$, cosolvent: 0.2 vol- $\% \mathrm{CH}_{3} \mathrm{CN}$, stopped-flow, detection at $607 \mathrm{~nm}$ )

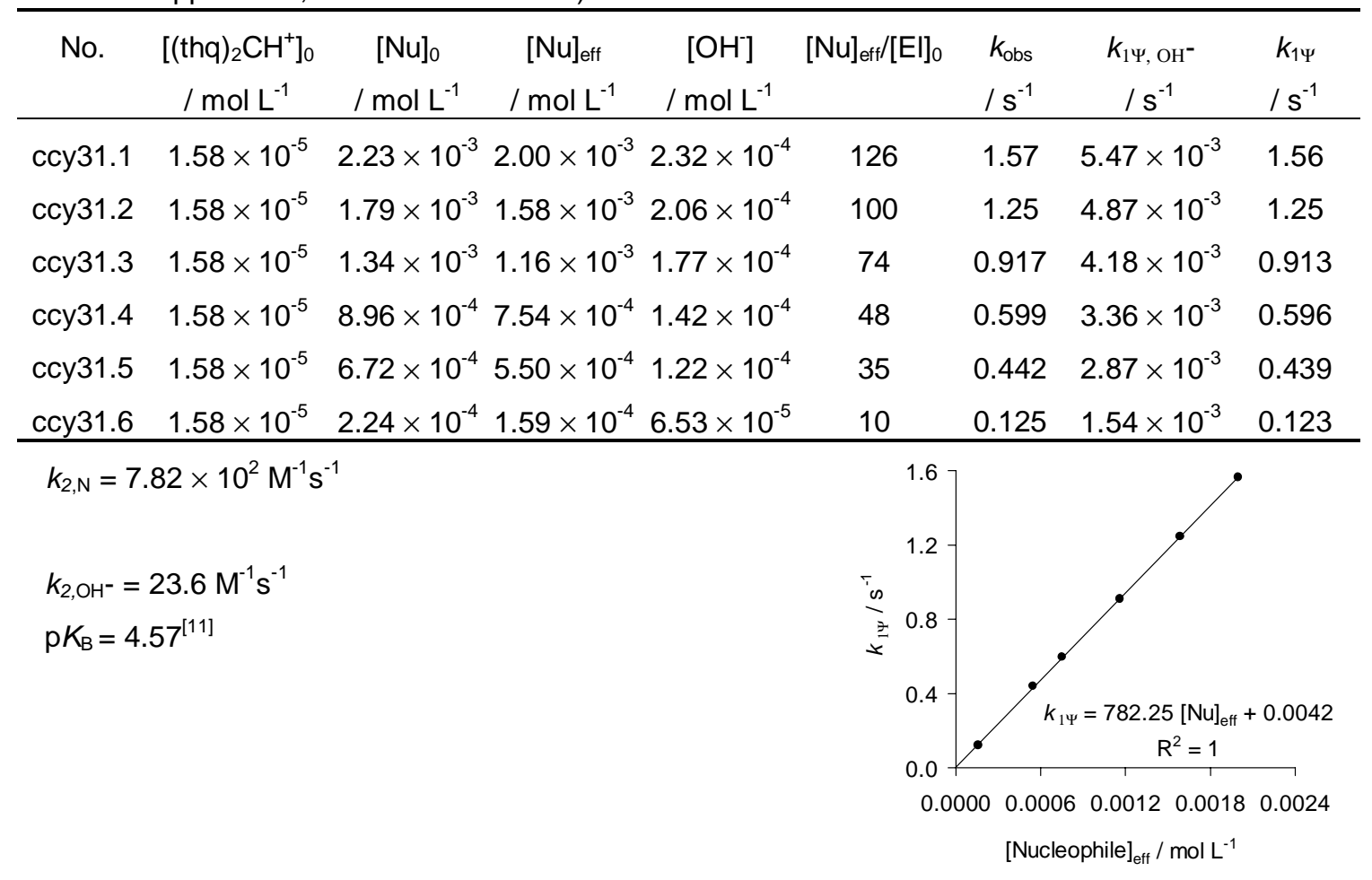


16.1.5. Reaction of Benzylamine (1n) with (ind) ${ }_{2} \mathrm{CH}^{+} \mathrm{BF}_{4}{ }^{-}$(at $20^{\circ} \mathrm{C}$, cosolvent: 0.2 vol- $\% \mathrm{CH}_{3} \mathrm{CN}$, stopped-flow, detection at $607 \mathrm{~nm}$ )

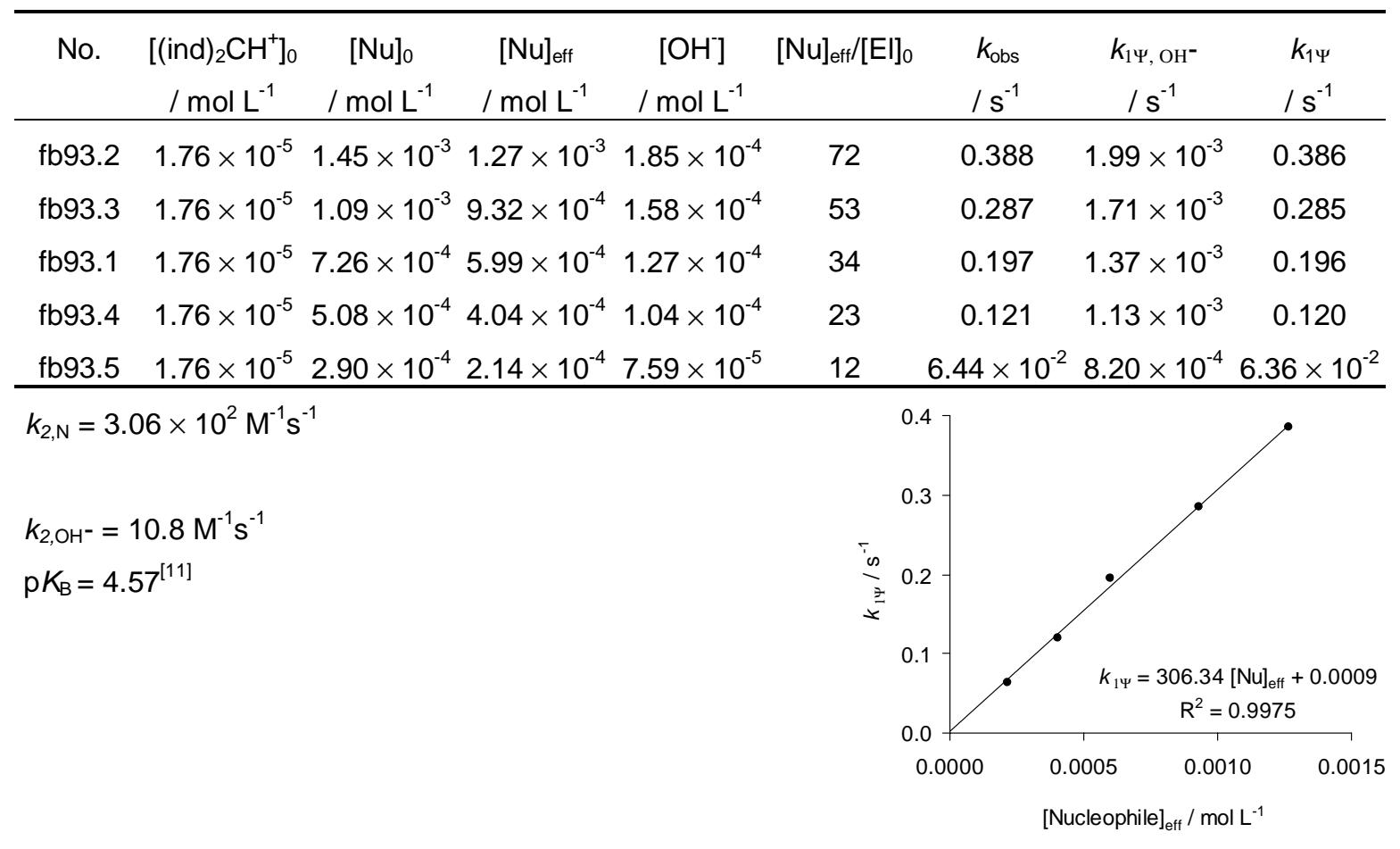

16.1.6. Reaction of Benzylamine (1n) with (jul) ${ }_{2} \mathrm{CH}^{+} \mathrm{BF}_{4}^{-}$(at $20^{\circ} \mathrm{C}$, cosolvent: 0.2 vol-\% $\mathrm{CH}_{3} \mathrm{CN}$, stopped-flow, detection at $630 \mathrm{~nm}$ )

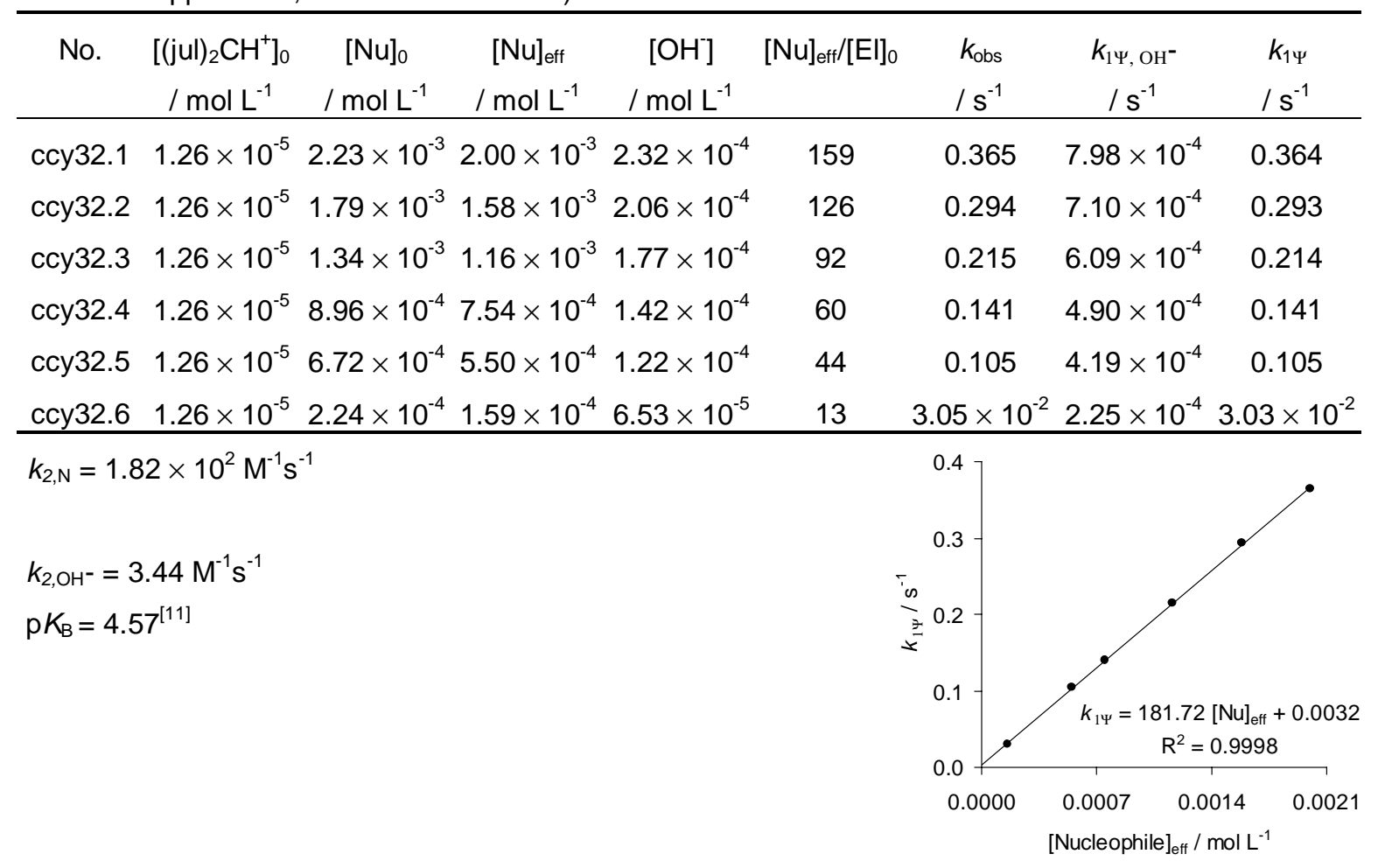


16.1.7. Reaction of Benzylamine (1n) with (lil) ${ }_{2} \mathrm{CH}^{+} \mathrm{BF}_{4}^{-}$(at $20^{\circ} \mathrm{C}$, cosolvent: 0.2 vol-\% $\mathrm{CH}_{3} \mathrm{CN}$, Stopped-flow and J\&M, detection at $634 \mathrm{~nm}$ )

\begin{tabular}{|c|c|c|c|c|c|c|c|c|}
\hline No. & $\begin{array}{c}{\left[(\mathrm{lil})_{2} \mathrm{CH}^{+}\right]_{0}} \\
/ \mathrm{mol} \mathrm{L}^{-1}\end{array}$ & $\begin{array}{c}{[\mathrm{Nu}]_{0}} \\
/ \mathrm{mol} \mathrm{L}^{-1}\end{array}$ & $\begin{array}{c}{[\mathrm{Nu}]_{\mathrm{eff}}} \\
/ \mathrm{mol} \mathrm{L}^{-1}\end{array}$ & $\begin{array}{c}{\left[\mathrm{OH}^{-}\right]} \\
/ \mathrm{mol} \mathrm{L}^{-1}\end{array}$ & {$[\mathrm{Nu}]_{\mathrm{efff}} /[\mathrm{El}]_{0}$} & $\begin{array}{l}k_{\mathrm{obs}} \\
/ \mathrm{s}^{-1}\end{array}$ & $\begin{array}{c}k_{1 \Psi, \mathrm{OH}^{-}} \\
/ \mathrm{s}^{-1} \\
\end{array}$ & $\begin{array}{l}k_{1 \Psi} \\
/ \mathrm{s}^{-1}\end{array}$ \\
\hline $\mathrm{fb} 95.1^{[\mathrm{a}]}$ & $2.66 \times 10^{-5}$ & $4.49 \times 10^{-3}$ & $4.16 \times 10^{-3}$ & $3.34 \times 10^{-4}$ & 156 & 0.392 & $7.2210^{-4}$ & 0.391 \\
\hline fb95.2 $2^{[a]}$ & $2.66 \times 10^{-5}$ & $3.14 \times 10^{-3}$ & $2.86 \times 10^{-3}$ & $2.78 \times 10^{-4}$ & 108 & 0.276 & $6.0010^{-4}$ & 0.275 \\
\hline $\mathrm{fb} 95.3^{[\mathrm{a}]}$ & $2.66 \times 10^{-5}$ & $2.24 \times 10^{-3}$ & $2.01 \times 10^{-3}$ & $2.32 \times 10^{-4}$ & 75 & 0.195 & $5.0210^{-4}$ & 0.194 \\
\hline fb95.4 $4^{[\mathrm{a}]}$ & $2.66 \times 10^{-5}$ & $1.35 \times 10^{-3}$ & $1.17 \times 10^{-3}$ & $1.78 \times 10^{-4}$ & 44 & 0.115 & $3.8410^{-4}$ & 0.115 \\
\hline fb94. $1^{[b]}$ & $1.07 \times 10^{-5}$ & $5.56 \times 10^{-4}$ & $4.46 \times 10^{-4}$ & $1.10 \times 10^{-4}$ & 42 & $4.40 \times 10^{-2}$ & $2.37 \times 10^{-4}$ & $4.38 \times 10^{-2}$ \\
\hline $\mathrm{fb} 94.4^{[\mathrm{b}]}$ & $1.07 \times 10^{-5}$ & $4.45 \times 10^{-4}$ & $3.48 \times 10^{-4}$ & $9.68 \times 10^{-5}$ & 33 & $3.46 \times 10^{-2}$ & $2.09 \times 10^{-4}$ & $3.44 \times 10^{-2}$ \\
\hline $\mathrm{fb} 94.3^{[b]}$ & $1.06 \times 10^{-5}$ & $2.21 \times 10^{-4}$ & $1.56 \times 10^{-4}$ & $6.48 \times 10^{-5}$ & 15 & $1.46 \times 10^{-2}$ & $1.40 \times 10^{-4}$ & $1.45 \times 10^{-2}$ \\
\hline fb95.5 $5^{[\mathrm{a}]}$ & $2.66 \times 10^{-5}$ & $4.49 \times 10^{-4}$ & $3.52 \times 10^{-4}$ & $9.73 \times 10^{-5}$ & 13 & $3.79 \times 10^{-2}$ & $2.10 \times 10^{-4}$ & $3.77 \times 10^{-2}$ \\
\hline $\mathrm{fb} 94.2^{[\mathrm{b}]}$ & $1.10 \times 10^{-5}$ & $1.15 \times 10^{-4}$ & $7.12 \times 10^{-5}$ & $4.38 \times 10^{-5}$ & 6 & $5.62 \times 10^{-3}$ & $9.46 \times 10^{-5}$ & $5.53 \times 10^{-3}$ \\
\hline
\end{tabular}

[a] Method: Stopped-flow

[b] Method: J\&M

$k_{2, \mathrm{~N}}=94.6 \mathrm{M}^{-1} \mathrm{~s}^{-1}$

$k_{2, \mathrm{OH}^{-}}=2.16 \mathrm{M}^{-1} \mathrm{~s}^{-1}$

$\mathrm{p} K_{\mathrm{B}}=4.57^{[11]}$

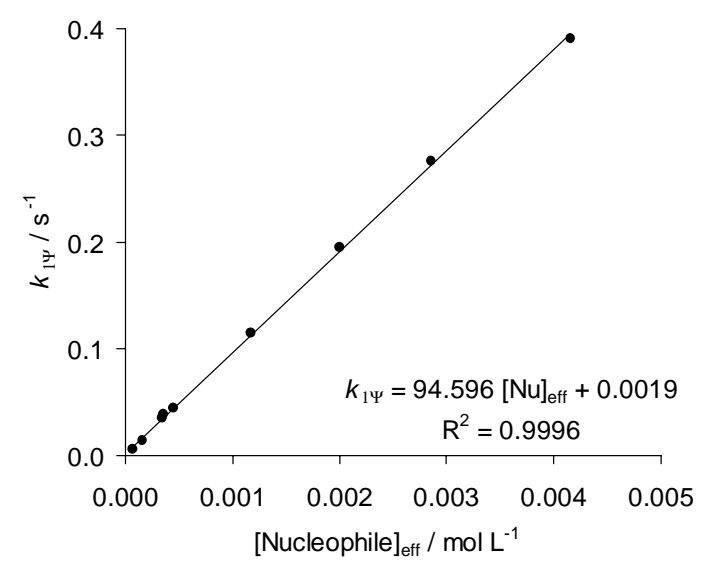

16.2. Reactivity parameters in water: $N=13.44 ; s=0.55$

\begin{tabular}{ccc}
\hline Reference electrophile & $E$ parameter & $k_{2, \mathrm{~N}}\left(20^{\circ} \mathrm{C}\right) / \mathrm{M}^{-1} \mathrm{~s}^{-1}$ \\
\hline (mor $)_{2} \mathrm{CH}^{+}$ & -5.53 & $3.13 \times 10^{4}$ \\
$(\mathrm{dma})_{2} \mathrm{CH}^{+}$ & -7.02 & $3.04 \times 10^{3}$ \\
$(\text { pyr })_{2} \mathrm{CH}^{+}$ & -7.69 & $1.29 \times 10^{3}$ \\
$(\text { thq })_{2} \mathrm{CH}^{+}$ & -8.22 & $7.82 \times 10^{2}$ \\
$(\text { ind })_{2} \mathrm{CH}^{+}$ & -8.76 & $3.06 \times 10^{2}$ \\
$(\mathrm{jul})_{2} \mathrm{CH}^{+}$ & -9.45 & $1.82 \times 10^{2}$ \\
$(\mathrm{lil})_{2} \mathrm{CH}^{+}$ & -10.04 & 94.6 \\
\hline
\end{tabular}

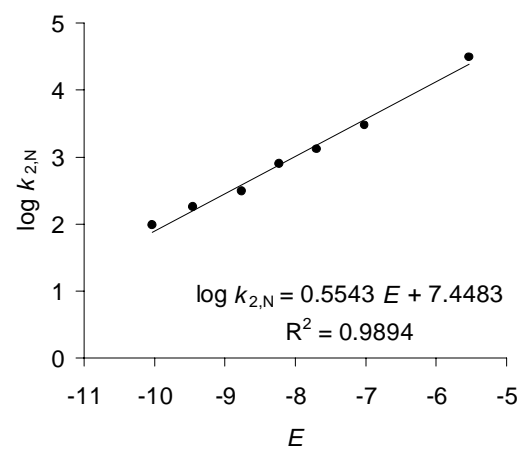




\section{4-Chloroaniline (10)}

17.1. Rate constants in Acetonitrile

17.1.1. Reaction of 4-Chloroaniline (10) with $\left(\right.$ mor) ${ }_{2} \mathrm{CH}^{+} \mathrm{BF}_{4}{ }^{-}$(at $20^{\circ} \mathrm{C}$, stopped-flow, detection at 620 $\mathrm{nm})$

\begin{tabular}{ccccc}
\hline No. & $\begin{array}{c}{\left[(\mathrm{mor})_{2} \mathrm{CH}^{+}\right]_{0}} \\
/ \mathrm{mol} \mathrm{L}^{-1}\end{array}$ & $\begin{array}{c}{[\mathrm{Nu}]_{0}} \\
/ \mathrm{mol} \mathrm{L}^{-1}\end{array}$ & {$[\mathrm{Nu}]_{0} /[\mathrm{El}]_{0}$} & $\begin{array}{c}k_{1 \Psi} \\
/ \mathrm{s}^{-1}\end{array}$ \\
\hline fb344.2 & $3.54 \times 10^{-5}$ & $5.05 \times 10^{-3}$ & 143 & $1.66 \times 10^{2}$ \\
fb344.3 & $3.54 \times 10^{-5}$ & $3.54 \times 10^{-3}$ & 100 & $1.16 \times 10^{2}$ \\
fb344.4 & $3.54 \times 10^{-5}$ & $2.02 \times 10^{-3}$ & 57 & 67.4 \\
fb344.5 & $3.54 \times 10^{-5}$ & $1.01 \times 10^{-3}$ & 29 & 32.7 \\
\hline
\end{tabular}

$k_{2, \mathrm{~N}}=3.29 \times 10^{4} \mathrm{M}^{-1} \mathrm{~s}^{-1}$

17.1.2. Reaction of 4-Chloroaniline (10) with $(\mathrm{dma})_{2} \mathrm{CH}^{+} \mathrm{BF}_{4}^{-}$(at $20^{\circ} \mathrm{C}$, stopped-flow, detection at $620 \mathrm{~nm})$

\begin{tabular}{ccccc}
\hline No. & $\begin{array}{c}{\left[(\mathrm{dma})_{2} \mathrm{CH}^{+}\right]_{0}} \\
/ \mathrm{mol} \mathrm{L}^{-1}\end{array}$ & $\begin{array}{c}{[\mathrm{Nu}]_{0}} \\
/ \mathrm{mol} \mathrm{L}^{-1}\end{array}$ & {$[\mathrm{Nu}]_{0} /[\mathrm{El}]_{0}$} & $\begin{array}{c}k_{1 \Psi} \\
/ \mathrm{s}^{-1}\end{array}$ \\
\hline j018.1 & $3.35 \times 10^{-5}$ & $1.01 \times 10^{-2}$ & 301 & 26.3 \\
j018.2 & $3.35 \times 10^{-5}$ & $5.05 \times 10^{-3}$ & 151 & 13.2 \\
j018.3 & $3.35 \times 10^{-5}$ & $3.54 \times 10^{-3}$ & 106 & 9.05 \\
j018.4 & $3.35 \times 10^{-5}$ & $2.02 \times 10^{-3}$ & 60 & 4.91 \\
j018.5 & $3.35 \times 10^{-5}$ & $1.01 \times 10^{-3}$ & 30 & 2.47 \\
j018.6 & $3.35 \times 10^{-5}$ & $5.05 \times 10^{-4}$ & 15 & 1.07 \\
\hline
\end{tabular}

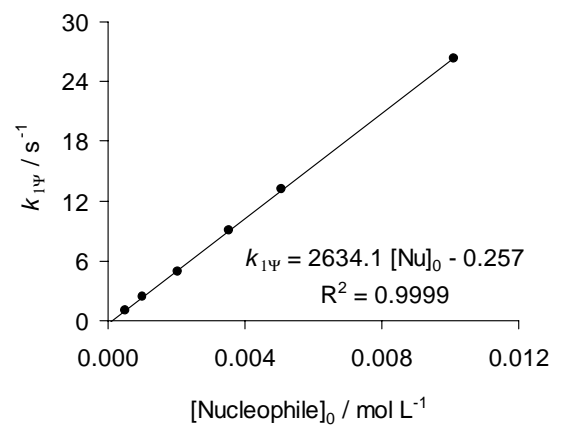

$k_{2, \mathrm{~N}}=2.63 \times 10^{3} \mathrm{M}^{-1} \mathrm{~s}^{-1}$

17.1.3. Reaction of 4-Chloroaniline (10) with (pyr) $)_{2} \mathrm{CH}^{+} \mathrm{BF}_{4}{ }_{4}^{-}$(at $20^{\circ} \mathrm{C}$, stopped-flow, detection at 620 $\mathrm{nm})$

\begin{tabular}{ccccc}
\hline No. & $\begin{array}{c}{\left[(\mathrm{pyr})_{2} \mathrm{CH}^{+}\right]_{0}} \\
/ \mathrm{mol} \mathrm{L}^{-1}\end{array}$ & $\begin{array}{c}{[\mathrm{Nu}]_{0}} \\
/ \mathrm{mol} \mathrm{L}^{-1}\end{array}$ & {$[\mathrm{Nu}]_{0} /[\mathrm{El}]_{0}$} & $\begin{array}{c}k_{1 \Psi} \\
/ \mathrm{s}^{-1}\end{array}$ \\
\hline fb345.2 & $3.57 \times 10^{-5}$ & $5.05 \times 10^{-3}$ & 141 & 9.36 \\
fb345.3 & $3.57 \times 10^{-5}$ & $3.54 \times 10^{-3}$ & 99 & 6.39 \\
fb345.4 & $3.57 \times 10^{-5}$ & $2.02 \times 10^{-3}$ & 57 & 3.73 \\
fb345.5 & $3.57 \times 10^{-5}$ & $1.01 \times 10^{-3}$ & 28 & 1.84 \\
\hline
\end{tabular}

$k_{2, \mathrm{~N}}=1.85 \times 10^{3} \mathrm{M}^{-1} \mathrm{~s}^{-1}$

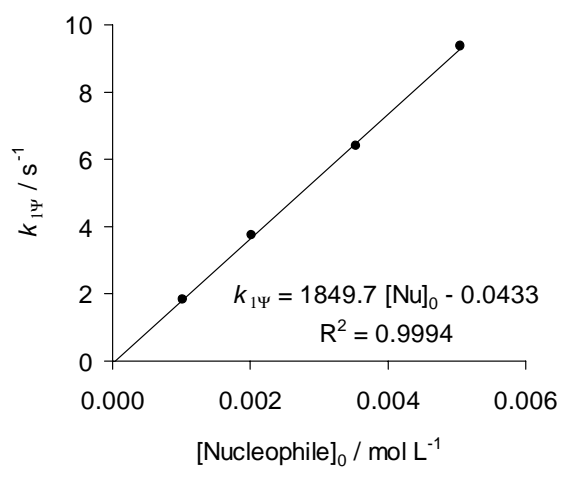


17.2. Reactivity parameters in Acetonitrile: $N=12.92 ; s=0.60$

\begin{tabular}{ccc}
\hline Reference electrophile & $E$ parameter & $k_{2, \mathrm{~N}}\left(20^{\circ} \mathrm{C}\right) / \mathrm{M}^{-1} \mathrm{~s}^{-1}$ \\
\hline$(\mathrm{mor})_{2} \mathrm{CH}^{+}$ & -5.53 & $3.29 \times 10^{4}$ \\
$(\mathrm{dma})_{2} \mathrm{CH}^{+}$ & -7.02 & $2.63 \times 10^{3}$ \\
$(\mathrm{pyr})_{2} \mathrm{CH}^{+}$ & -7.69 & $1.85 \times 10^{3}$ \\
\hline
\end{tabular}

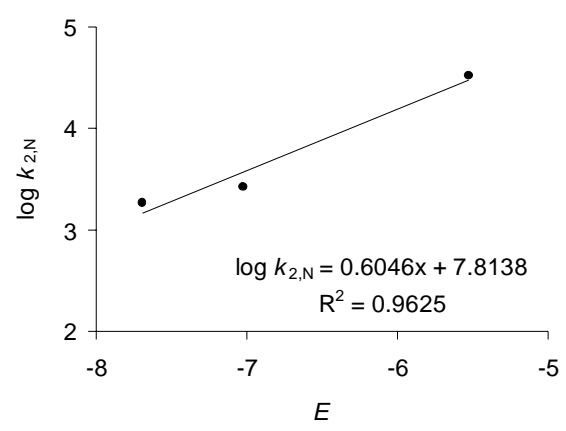

\section{Aniline (1p)}

18.1. Rate constants in Acetonitrile

18.1.1. Reaction of Aniline (1p) with (mor) $)_{2} \mathrm{CH}^{+} \mathrm{BF}_{4}^{-}$(at $20^{\circ} \mathrm{C}$, stopped-flow, detection at $620 \mathrm{~nm}$ )

\begin{tabular}{ccccc}
\hline No. & $\begin{array}{c}{\left[(\mathrm{mor})_{2} \mathrm{CH}^{+}\right]_{0}} \\
/ \mathrm{mol} \mathrm{L}^{-1}\end{array}$ & $\begin{array}{c}{[\mathrm{Nu}]_{0}} \\
/ \mathrm{mol} \mathrm{L}^{-1}\end{array}$ & {$[\mathrm{Nu}]_{0} /[\mathrm{El}]_{0}$} & $\begin{array}{c}k_{1 \Psi} \\
/ \mathrm{s}^{-1}\end{array}$ \\
\hline j016.1 & $5.30 \times 10^{-5}$ & $5.10 \times 10^{-3}$ & 96 & $3.67 \times 10^{2}$ \\
j016.2 & $5.30 \times 10^{-5}$ & $3.57 \times 10^{-3}$ & 67 & $2.65 \times 10^{2}$ \\
j016.3 & $5.30 \times 10^{-5}$ & $2.04 \times 10^{-3}$ & 38 & $1.70 \times 10^{2}$ \\
j016.4 & $5.30 \times 10^{-5}$ & $1.02 \times 10^{-3}$ & 19 & 79.4 \\
j016.5 & $5.30 \times 10^{-5}$ & $5.10 \times 10^{-4}$ & 10 & 45.4 \\
\hline
\end{tabular}

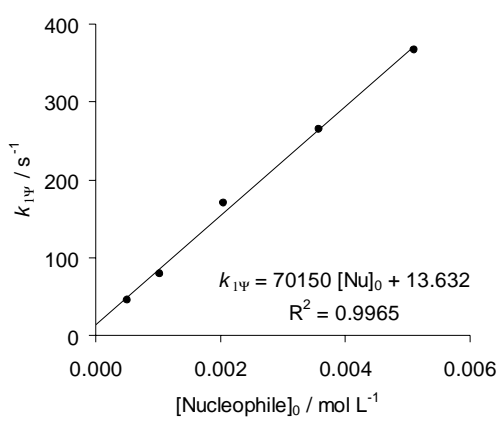

$k_{2, \mathrm{~N}}=7.02 \times 10^{4} \mathrm{M}^{-1} \mathrm{~s}^{-1}$

18.1.2. Reaction of Aniline (1p) with (dma) ${ }_{2} \mathrm{CH}^{+} \mathrm{BF}_{4}{ }^{-}$(at $20{ }^{\circ} \mathrm{C}$, stopped-flow, detection at $610 \mathrm{~nm}$ )

\begin{tabular}{ccccc} 
No. & $\begin{array}{c}{\left[(\mathrm{dma})_{2} \mathrm{CH}^{+}\right]_{0}} \\
/ \mathrm{mol} \mathrm{L}^{-1}\end{array}$ & $\begin{array}{c}{[\mathrm{Nu}]_{0}} \\
/ \mathrm{mol} \mathrm{L}^{-1}\end{array}$ & {$[\mathrm{Nu}]_{0} /[\mathrm{El}]_{0}$} & $\begin{array}{c}k_{1 \Psi} \\
/ \mathrm{s}^{-1}\end{array}$ \\
\hline fb341.1 & $2.87 \times 10^{-5}$ & $1.02 \times 10^{-2}$ & 355 & 73.1 \\
fb341.2 & $2.87 \times 10^{-5}$ & $5.10 \times 10^{-3}$ & 178 & 36.8 \\
fb341.3 & $2.87 \times 10^{-5}$ & $3.57 \times 10^{-3}$ & 124 & 25.7 \\
fb341.4 & $2.87 \times 10^{-5}$ & $2.04 \times 10^{-3}$ & 71 & 14.9 \\
fb341.5 & $2.87 \times 10^{-5}$ & $1.02 \times 10^{-3}$ & 36 & 7.29 \\
fb341.6 & $2.87 \times 10^{-5}$ & $5.10 \times 10^{-4}$ & 18 & 3.66 \\
\hline
\end{tabular}

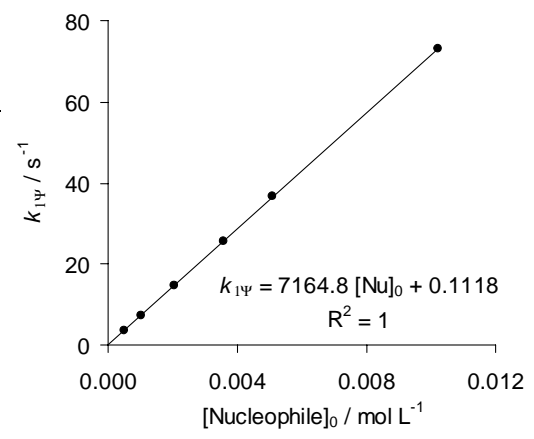

$k_{2, \mathrm{~N}}=7.16 \times 10^{3} \mathrm{M}^{-1} \mathrm{~s}^{-1}$ 
18.1.3. Reaction of Aniline (1p) with (pyr) $)_{2} \mathrm{CH}^{+} \mathrm{BF}_{4}^{-}$(at $20^{\circ} \mathrm{C}$, stopped-flow, detection at $610 \mathrm{~nm}$ )

\begin{tabular}{ccccc} 
No. & $\begin{array}{c}{\left[(\mathrm{pyr})_{2} \mathrm{CH}^{+}\right]_{0}} \\
/ \mathrm{mol} \mathrm{L}^{-1}\end{array}$ & $\begin{array}{c}{[\mathrm{Nu}]_{0}} \\
/ \mathrm{mol} \mathrm{L}^{-1}\end{array}$ & {$[\mathrm{Nu}]_{0} /[\mathrm{El}]_{0}$} & $\begin{array}{c}k_{1 \Psi} \\
/ \mathrm{s}^{-1}\end{array}$ \\
\hline fb343.1 & $3.57 \times 10^{-5}$ & $1.02 \times 10^{-2}$ & 286 & 32.2 \\
fb343.2 & $3.57 \times 10^{-5}$ & $5.10 \times 10^{-3}$ & 143 & 16.5 \\
fb343.3 & $3.57 \times 10^{-5}$ & $3.57 \times 10^{-3}$ & 100 & 11.6 \\
fb343.4 & $3.57 \times 10^{-5}$ & $2.04 \times 10^{-3}$ & 57 & 6.82 \\
fb343.5 & $3.57 \times 10^{-5}$ & $1.02 \times 10^{-3}$ & 29 & 3.31 \\
fb343.6 & $3.57 \times 10^{-5}$ & $5.10 \times 10^{-4}$ & 14 & 1.75 \\
\hline
\end{tabular}

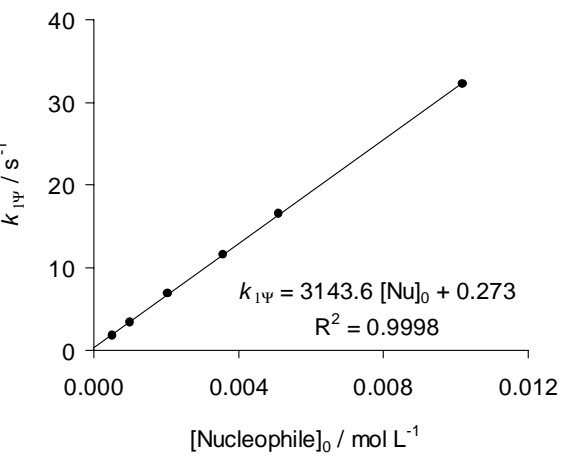

$k_{2, \mathrm{~N}}=3.14 \times 10^{3} \mathrm{M}^{-1} \mathrm{~s}^{-1}$

18.1.4. Reaction of Aniline (1p) with (thq) ${ }_{2} \mathrm{CH}^{+} \mathrm{BF}_{4}{ }^{-}$(at $20^{\circ} \mathrm{C}$, stopped-flow, detection at $620 \mathrm{~nm}$ )

\begin{tabular}{ccccc} 
No. & $\begin{array}{c}\left.[\text { (thq })_{2} \mathrm{CH}^{+}\right]_{0} \\
/ \mathrm{mol} \mathrm{L}^{-1}\end{array}$ & $\begin{array}{c}{[\mathrm{Nu}]_{0}} \\
/ \mathrm{mol} \mathrm{L}^{-1}\end{array}$ & {$[\mathrm{Nu}]_{0} /[\mathrm{El}]_{0}$} & $\begin{array}{c}k_{1 \Psi} \\
/ \mathrm{s}^{-1}\end{array}$ \\
\hline j015.1 & $7.39 \times 10^{-5}$ & $5.10 \times 10^{-3}$ & 69 & 4.40 \\
j015.2 & $7.39 \times 10^{-5}$ & $3.57 \times 10^{-3}$ & 48 & 3.05 \\
j015.3 & $7.39 \times 10^{-5}$ & $2.04 \times 10^{-3}$ & 28 & 1.71 \\
j015.4 & $7.39 \times 10^{-5}$ & $1.02 \times 10^{-3}$ & 14 & 0.791 \\
j015.5 & $7.39 \times 10^{-5}$ & $5.10 \times 10^{-4}$ & 7 & 0.439 \\
\hline
\end{tabular}

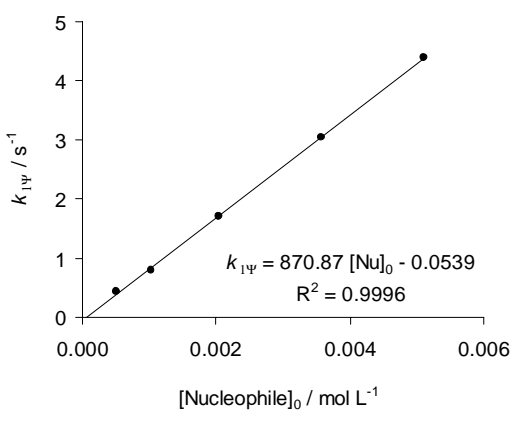

$k_{2, \mathrm{~N}}=8.71 \times 10^{2} \mathrm{M}^{-1} \mathrm{~s}^{-1}$

18.2. Reactivity parameters in Acetonitrile: $N=12.64 ; s=0.68$

\begin{tabular}{ccc}
\hline Reference electrophile & $E$ parameter & $k_{2, \mathrm{~N}}\left(20^{\circ} \mathrm{C}\right) / \mathrm{M}^{-1} \mathrm{~s}^{-1}$ \\
\hline (mor $)_{2} \mathrm{CH}^{+}$ & -5.53 & $7.02 \times 10^{4}$ \\
$(\mathrm{dma})_{2} \mathrm{CH}^{+}$ & -7.02 & $7.16 \times 10^{3}$ \\
$(\text { pyr })_{2} \mathrm{CH}^{+}$ & -7.69 & $3.14 \times 10^{3}$ \\
(thq $)_{2} \mathrm{CH}^{+}$ & -8.22 & $8.71 \times 10^{2}$ \\
\hline
\end{tabular}

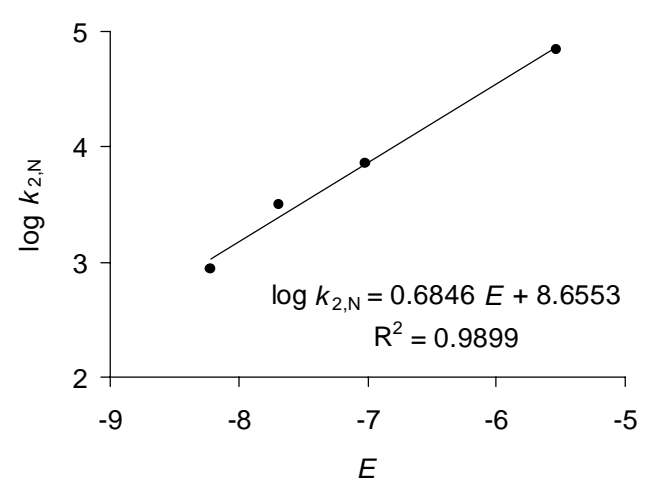


18.3. Rate constants and equilibrium constants in water

18.3.1. Reaction of Aniline (1p) with (mor) ${ }_{2} \mathrm{CH}^{+} \mathrm{BF}_{4}^{-}$(at $20^{\circ} \mathrm{C}$, cosolvent: 9 vol-\% $\mathrm{CH}_{3} \mathrm{CN}$, stoppedflow, detection at $\left.614 \mathrm{~nm},[\mathrm{Nu}]_{\mathrm{eff}}=[\mathrm{Nu}]_{0}, k_{\mathrm{obs}}=k_{1 \Psi}\right)$

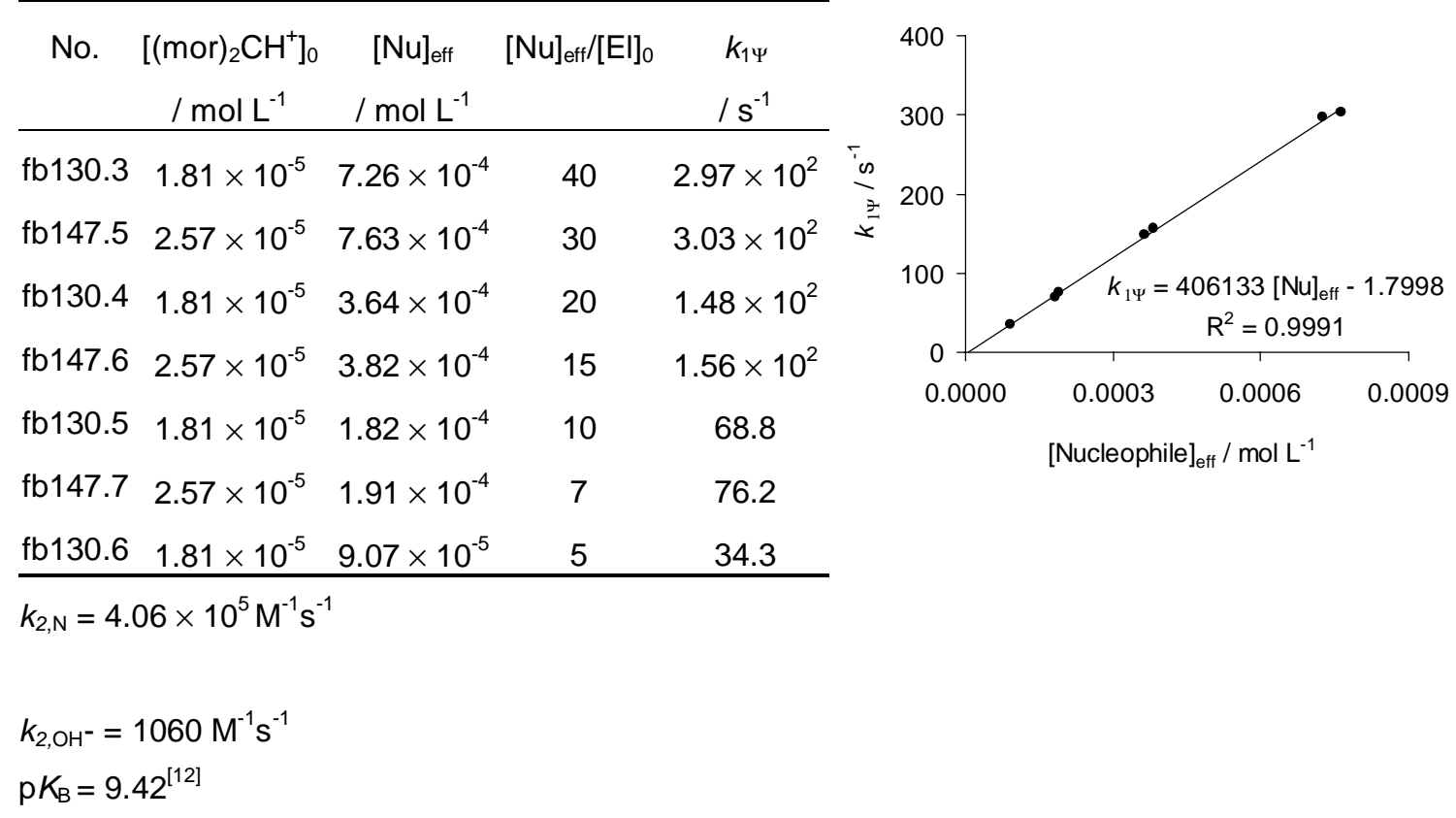

18.3.2. Reaction of Aniline (1p) with (dma) ${ }_{2} \mathrm{CH}^{+} \mathrm{BF}_{4}^{-}$(at $20^{\circ} \mathrm{C}$, cosolvent: 0.1 vol- $\% \mathrm{CH}_{3} \mathrm{CN}$, stopped-flow, detection at $\left.614 \mathrm{~nm},[\mathrm{Nu}]_{\text {eff }}=[\mathrm{Nu}]_{0}, k_{\mathrm{obs}}=k_{1 \Psi}\right)$

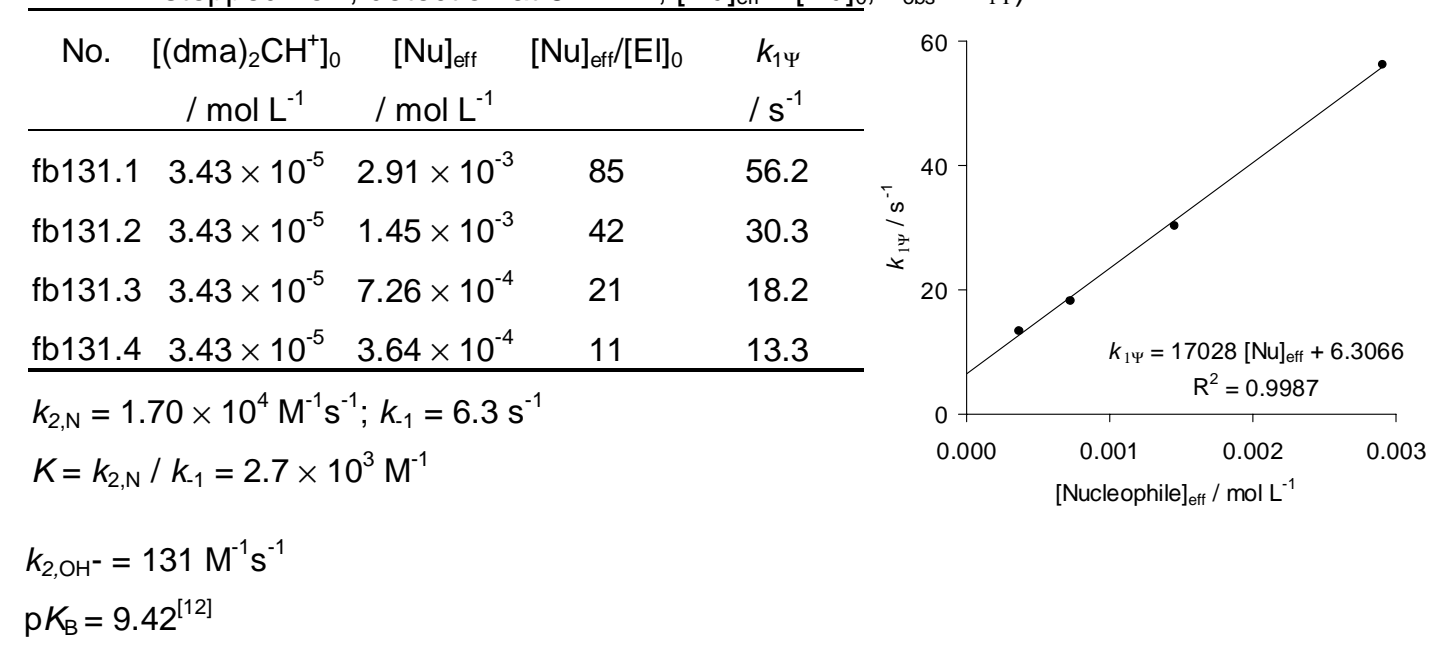


18.3.3. Reaction of Aniline (1p) with (pyr) $)_{2} \mathrm{CH}^{+} \mathrm{BF}_{4}{ }_{4}$ (at $20^{\circ} \mathrm{C}$, cosolvent: 0.1 vol- $\% \mathrm{CH}_{3} \mathrm{CN}$, stoppedflow, detection at $612 \mathrm{~nm}$, $\left.[\mathrm{Nu}]_{\mathrm{eff}}=[\mathrm{Nu}]_{0}, k_{\mathrm{obs}}=k_{1 \Psi}\right)$

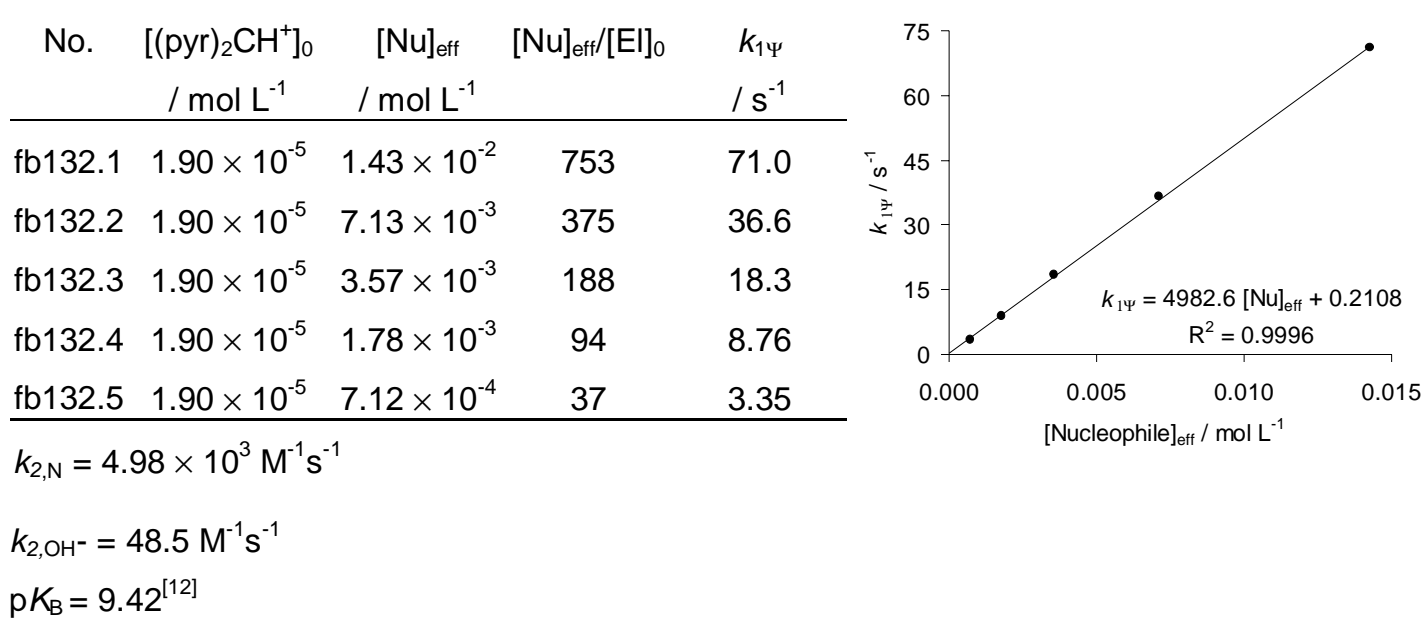

18.3.4. Reaction of Aniline (1p) with (thq) ${ }_{2} \mathrm{CH}^{+} \mathrm{BF}_{4}{ }^{-}$(at $20{ }^{\circ} \mathrm{C}$, cosolvent: 0.4 vol-\% $\mathrm{CH}_{3} \mathrm{CN}$, stoppedflow, detection at $\left.611 \mathrm{~nm},[\mathrm{Nu}]_{\text {eff }}=[\mathrm{Nu}]_{0}, k_{\mathrm{obs}}=k_{1 \Psi}\right)$

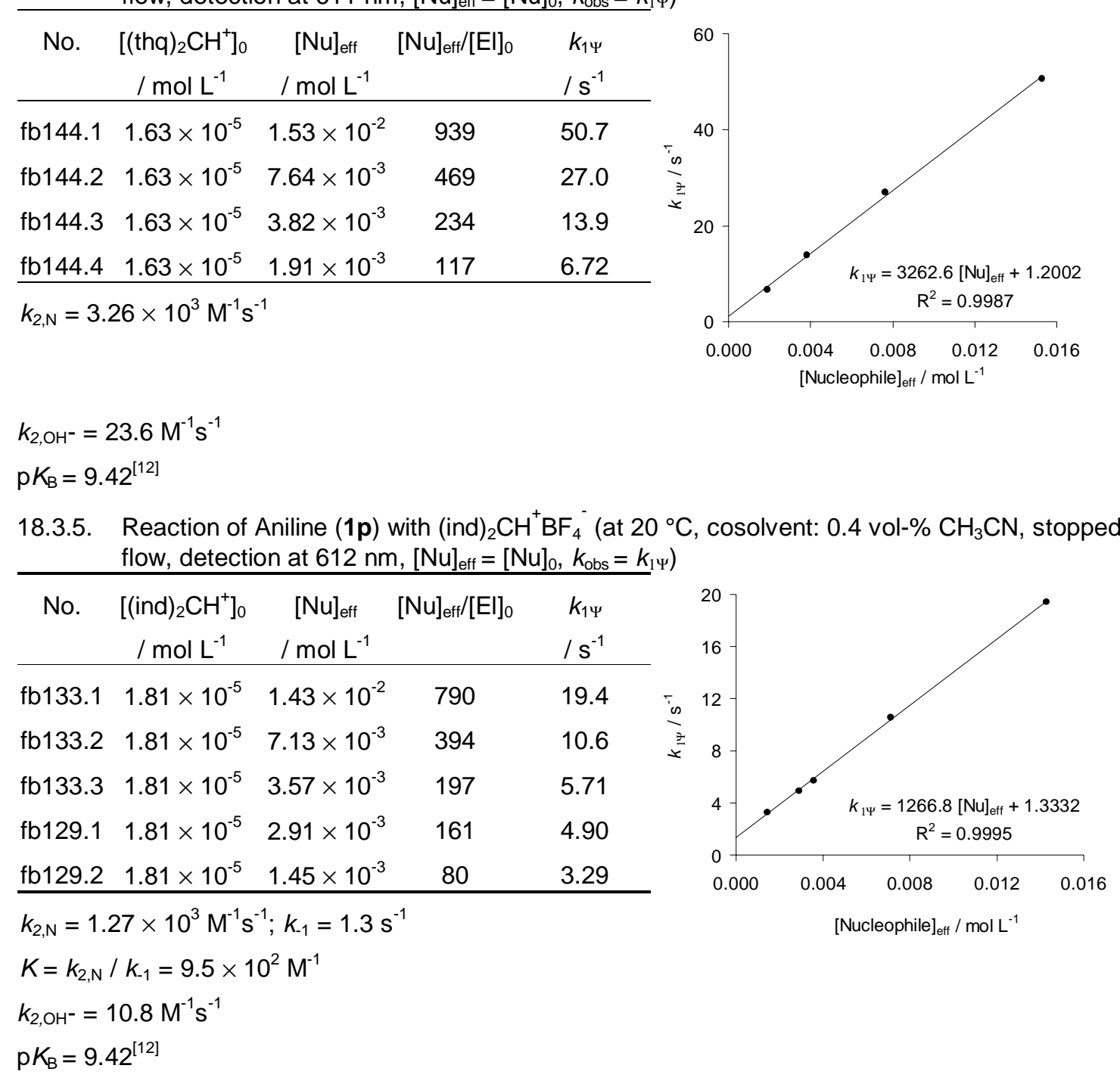


18.4. Rate constants in water at $\mathrm{pH}=11$

18.4.1. Reaction of Aniline (1p) with (thq) ${ }_{2} \mathrm{CH}^{+} \mathrm{BF}_{4}{ }^{-}\left(\mathrm{pH}=11\right.$, at $20{ }^{\circ} \mathrm{C}$, cosolvent: 0.4 vol- $\% \mathrm{CH}_{3} \mathrm{CN}$, stopped-flow, detection at $611 \mathrm{~nm}$, $\left.[\mathrm{Nu}]_{\text {eff }}=[\mathrm{Nu}]_{0}, k_{\mathrm{obs}}=k_{1 \Psi}\right)$

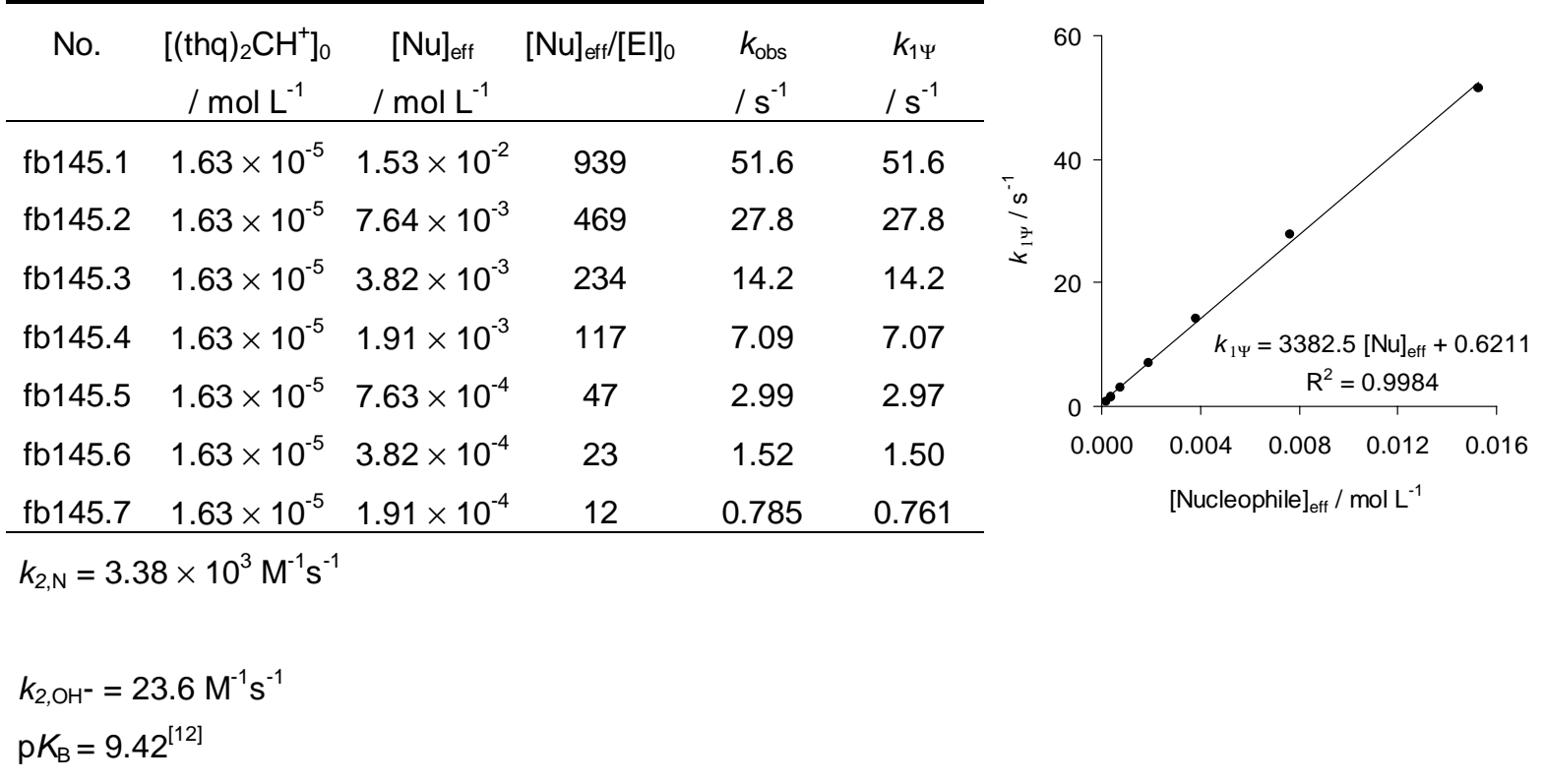

18.4.2. Reaction of Aniline (1p) with $(\mathrm{jul})_{2} \mathrm{CH}^{+} \mathrm{BF}_{4}^{-}\left(\mathrm{pH}=11\right.$, at $20^{\circ} \mathrm{C}$, cosolvent: 0.4 vol- $\% \mathrm{CH}_{3} \mathrm{CN}$, stopped-flow, detection at $625 \mathrm{~nm}$, $\left.[\mathrm{Nu}]_{\text {eff }}=[\mathrm{Nu}]_{0}, k_{\mathrm{obs}}=k_{1} \Psi\right)$

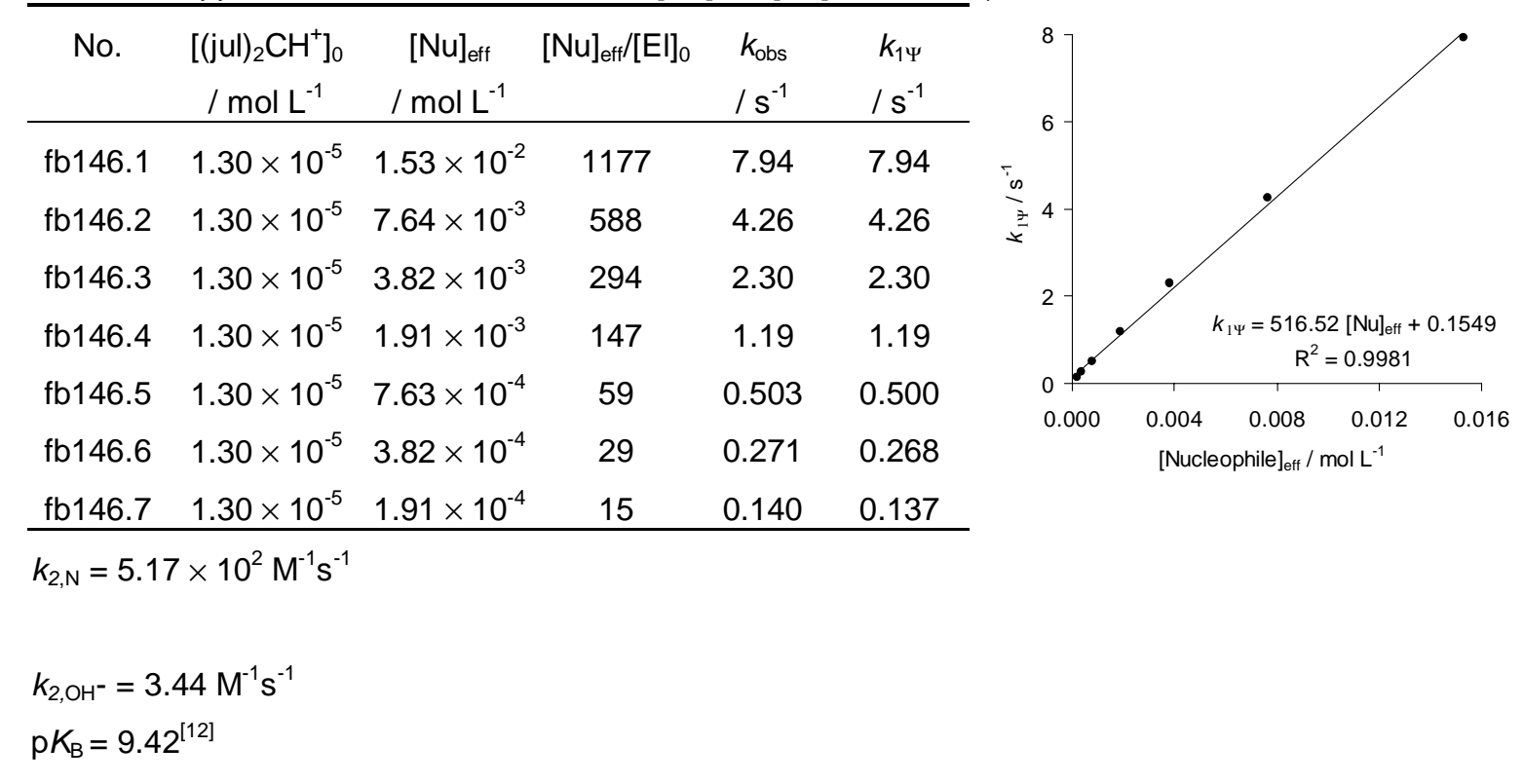


18.5. $\mathrm{pH}$ dependance of rate constants for the reaction of Aniline (1p) with $(\mathrm{dma})_{2} \mathrm{CH}^{+} \mathrm{BF}_{4}{ }^{-}$

(phosphate buffer, at $20{ }^{\circ} \mathrm{C}$, cosolvent: 9 vol- $\% \mathrm{CH}_{3} \mathrm{CN}$, stopped-flow, detection at $614 \mathrm{~nm}$, $\mathrm{pH}$ measured, $[\mathrm{Nu}]_{\mathrm{eff}}=[\mathrm{Nu}]_{0}, k_{\mathrm{obs}}=k_{1 \Psi}, k_{1 \Psi, \mathrm{OH}-}=k_{2, \mathrm{OH}-}\left[\mathrm{OH}^{-}\right]$calculated)

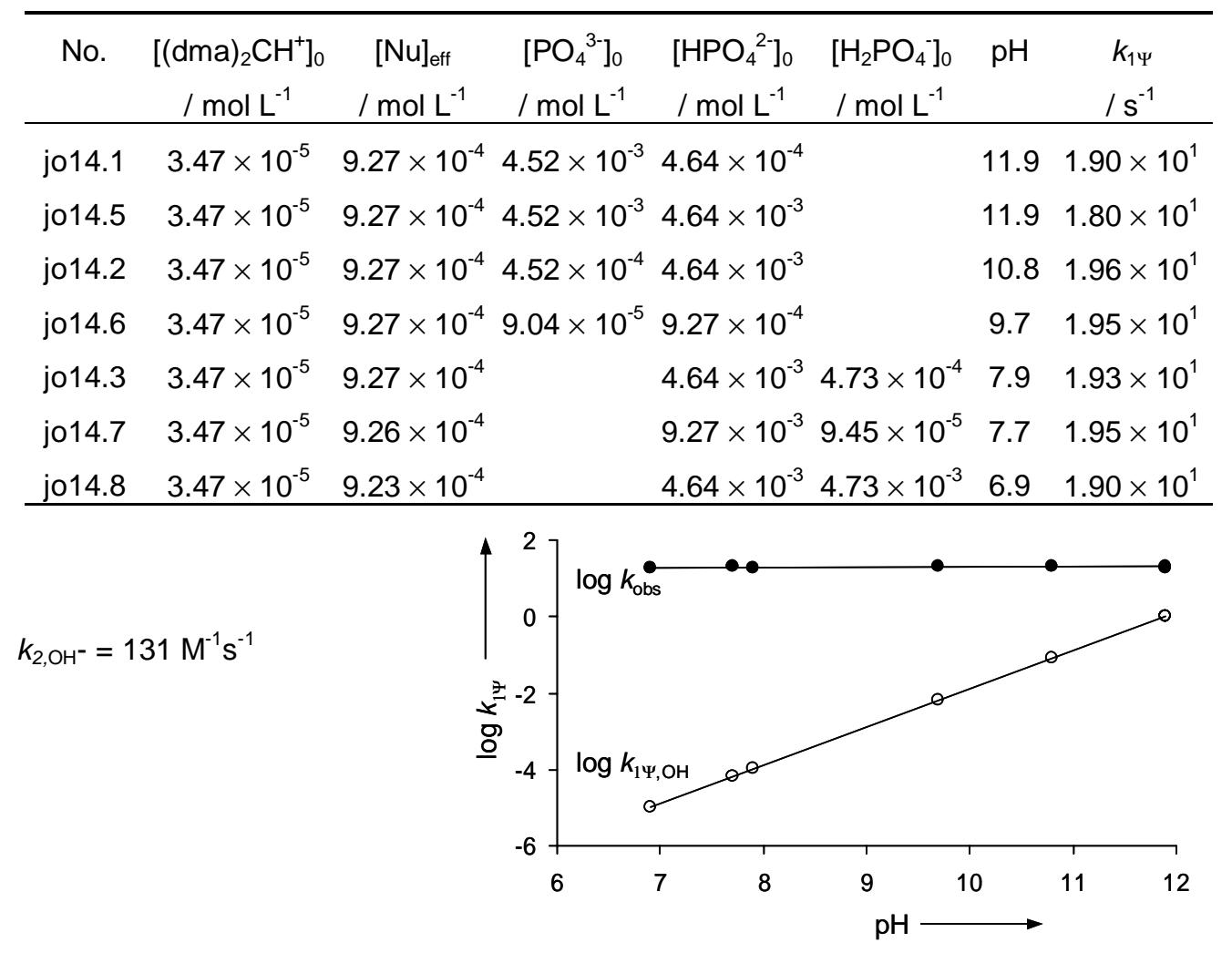

18.6. Reactivity parameters in water: $N=12.99 ; s=0.73$

\begin{tabular}{ccc}
\hline Reference electrophile & $E$ parameter & $k_{2, \mathrm{~N}}\left(20^{\circ} \mathrm{C}\right) / \mathrm{M}^{-1} \mathrm{~s}^{-1}$ \\
\hline (mor $)_{2} \mathrm{CH}^{+}$ & -5.53 & $4.06 \times 10^{5}$ \\
$(\mathrm{dma})_{2} \mathrm{CH}^{+}$ & -7.02 & $1.70 \times 10^{4}$ \\
$(\mathrm{pyr})_{2} \mathrm{CH}^{+}$ & -7.69 & $4.98 \times 10^{3}$ \\
$(\text { thq })_{2} \mathrm{CH}^{+}$ & -8.22 & $3.26 \times 10^{3}$ \\
$(\text { ind })_{2} \mathrm{CH}^{+}$ & -8.76 & $1.27 \times 10^{3}$ \\
$(\mathrm{jul})_{2} \mathrm{CH}^{+}$ & -9.45 & $5.17 \times 10^{2}$ \\
\hline
\end{tabular}

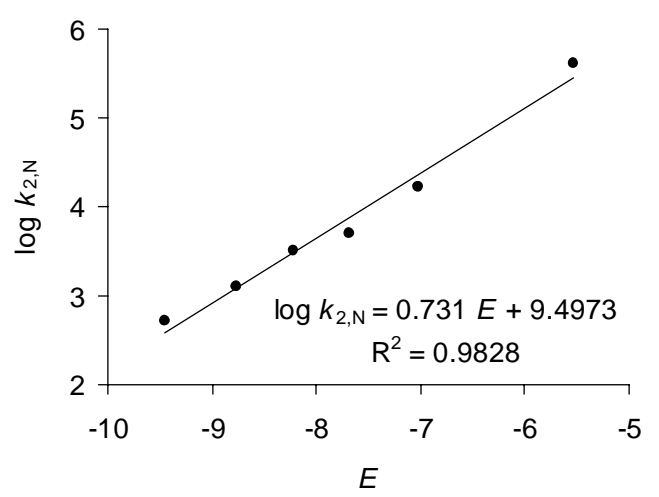




\section{9. $p$-Toluidine (1q)}

19.1. Rate constants in Acetonitrile

19.1.1. Reaction of $p$-Toluidine (1q) with (mor) ${ }_{2} \mathrm{CH}^{+} \mathrm{BF}_{4}{ }^{-}$(at $20^{\circ} \mathrm{C}$, stopped-flow, detection at 620 $\mathrm{nm})$

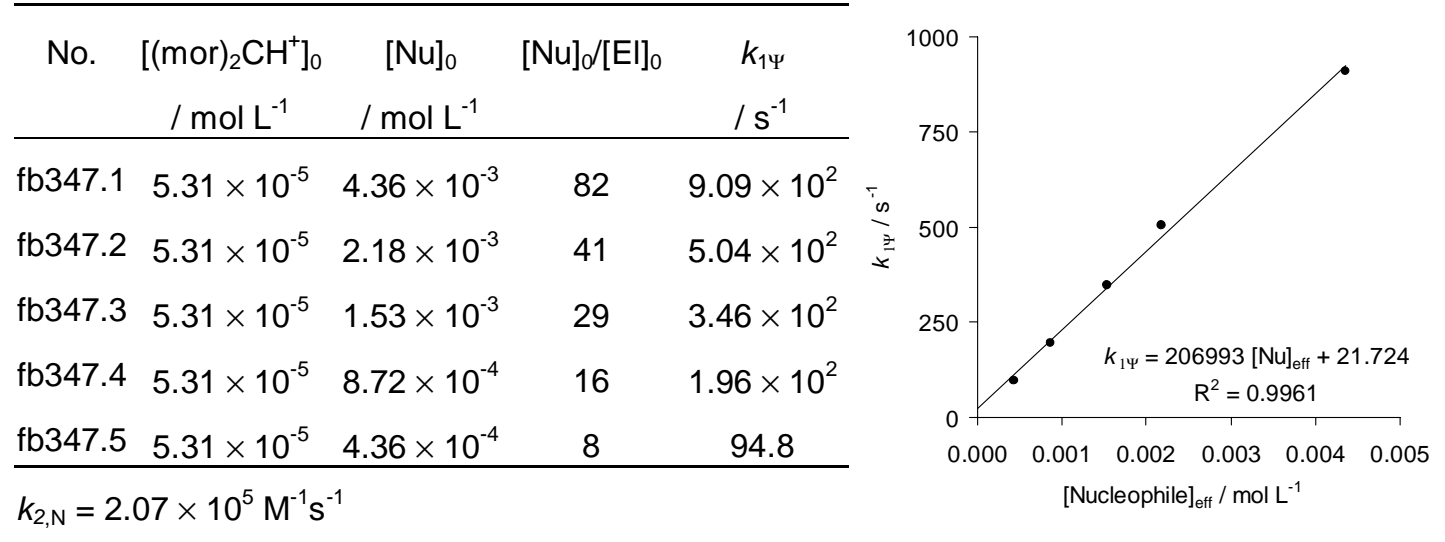

19.1.2. Reaction of $p$-Toluidine (1q) with $(\mathrm{dma})_{2} \mathrm{CH}^{+} \mathrm{BF}_{4}{ }_{4}$ (at $20^{\circ} \mathrm{C}$, stopped-flow, detection at 620 $\mathrm{nm})$

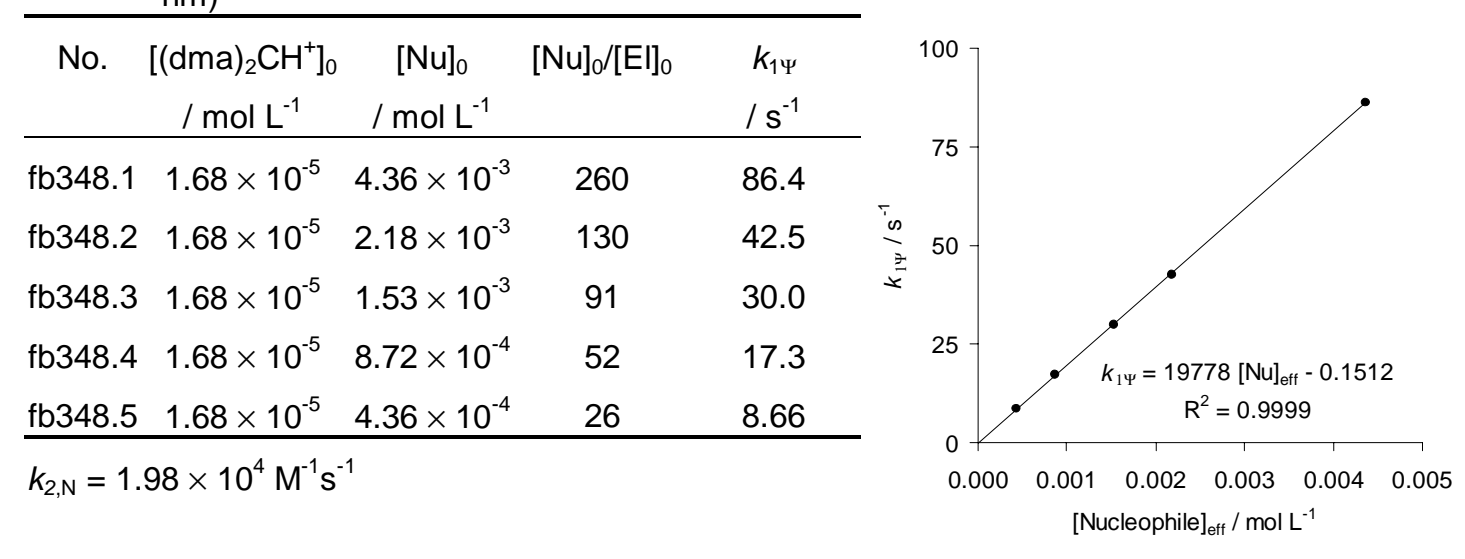

19.1.3. Reaction of $p$-Toluidine (1q) with $(\mathrm{pyr})_{2} \mathrm{CH}^{+} \mathrm{BF}_{4}{ }^{-}$(at $20^{\circ} \mathrm{C}$, stopped-flow, detection at $620 \mathrm{~nm}$ )

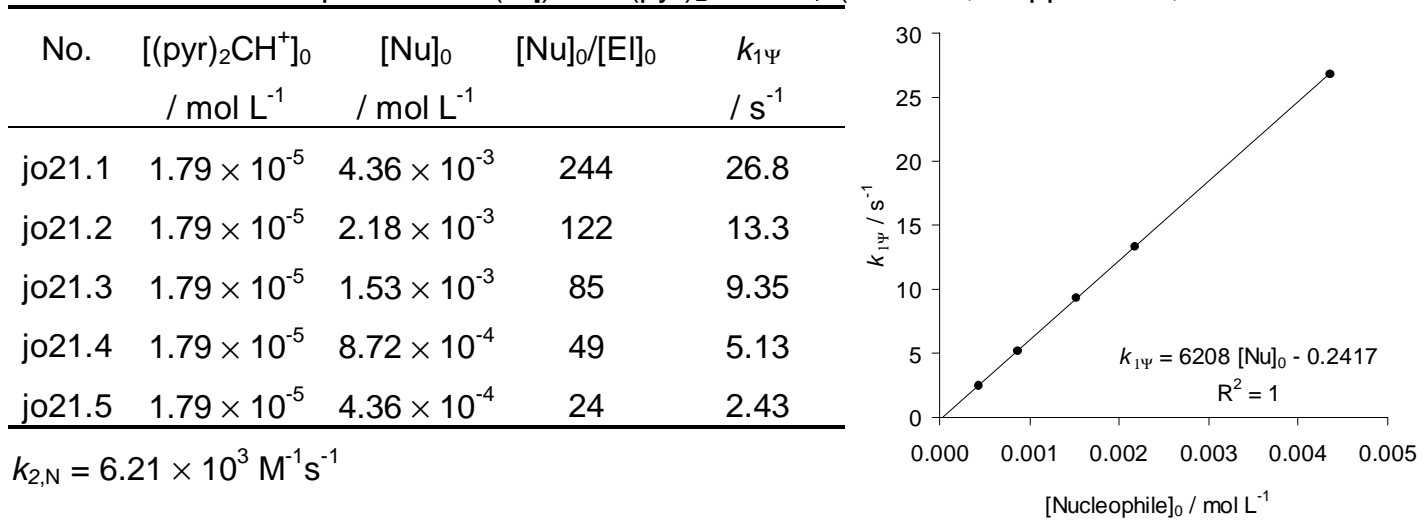


19.1.4. Reaction of p-Toluidine (1q) with (thq) ${ }_{2} \mathrm{CH}^{+} \mathrm{BF}_{4}{ }_{4}^{-}$(at $20^{\circ} \mathrm{C}$, stopped-flow, detection at $620 \mathrm{~nm}$ )

\begin{tabular}{ccccc} 
No. & $\begin{array}{c}{\left[(\text { thq })_{2} \mathrm{CH}^{+}\right]_{0}} \\
/ \mathrm{mol} \mathrm{L}^{-1}\end{array}$ & $\begin{array}{c}{[\mathrm{Nu}]_{0}} \\
/ \mathrm{mol} \mathrm{L}^{-1}\end{array}$ & {$[\mathrm{Nu}]_{0} /[\mathrm{El}]_{0}$} & $\begin{array}{c}k_{1 \Psi} \\
/ \mathrm{s}^{-1}\end{array}$ \\
\hline j020.1 & $1.35 \times 10^{-5}$ & $7.93 \times 10^{-3}$ & 587 & 22.8 \\
j020.2 & $1.35 \times 10^{-5}$ & $3.96 \times 10^{-3}$ & 293 & 11.3 \\
j020.3 & $1.35 \times 10^{-5}$ & $2.77 \times 10^{-3}$ & 205 & 7.86 \\
j020.4 & $1.35 \times 10^{-5}$ & $1.58 \times 10^{-3}$ & 117 & 4.49 \\
j020.5 & $1.35 \times 10^{-5}$ & $7.93 \times 10^{-4}$ & 59 & 2.02 \\
\hline
\end{tabular}

$k_{2, \mathrm{~N}}=2.90 \times 10^{3} \mathrm{M}^{-1} \mathrm{~s}^{-1}$

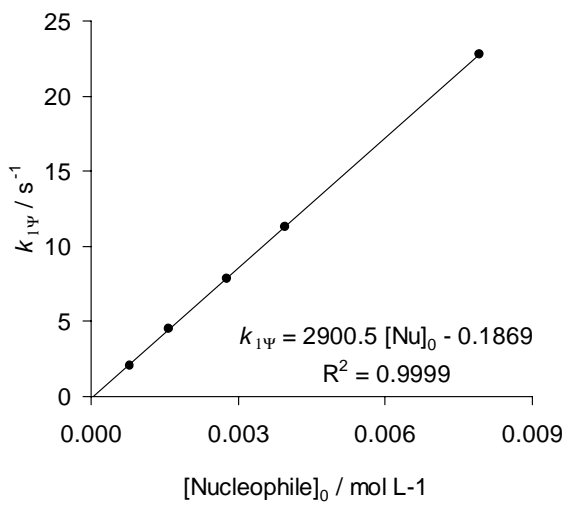

19.2. Reactivity parameters in Acetonitrile: $N=13.19 ; s=0.69$

\begin{tabular}{ccc}
\hline Reference electrophile & $E$ parameter & $k_{2, \mathrm{~N}}\left(20^{\circ} \mathrm{C}\right) / \mathrm{M}^{-1} \mathrm{~s}^{-1}$ \\
\hline$(\text { mor })_{2} \mathrm{CH}^{+}$ & -5.53 & $2.07 \times 10^{5}$ \\
$(\mathrm{dma})_{2} \mathrm{CH}^{+}$ & -7.02 & $1.98 \times 10^{4}$ \\
$(\text { pyr })_{2} \mathrm{CH}^{+}$ & -7.69 & $6.21 \times 10^{3}$ \\
$(\text { thq })_{2} \mathrm{CH}^{+}$ & -8.22 & $2.90 \times 10^{3}$ \\
\hline
\end{tabular}

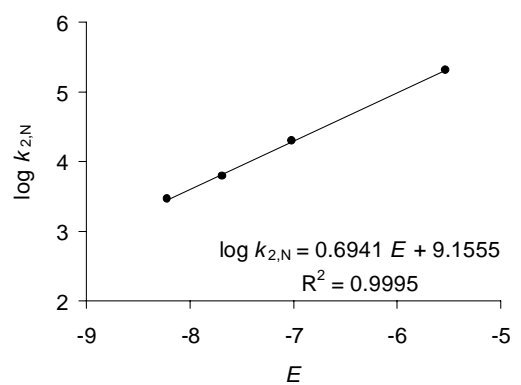

19.3. Rate constants in water

19.3.1. Reaction of $p$-Toluidine (1q) with (mor) ${ }_{2} \mathrm{CH}^{+} \mathrm{BF}_{4}^{-}$(at $20^{\circ} \mathrm{C}$, cosolvent: 9 vol- $\% \mathrm{CH}_{3} \mathrm{CN}$, stopped-flow, detection at $\left.611 \mathrm{~nm},[\mathrm{Nu}]_{\mathrm{eff}}=[\mathrm{Nu}]_{0}, k_{\mathrm{obs}}=k_{1 \Psi}\right)$

No. $\quad\left[(\mathrm{mor})_{2} \mathrm{CH}^{+}\right]_{0} \quad[\mathrm{Nu}]_{\mathrm{eff}} \quad[\mathrm{Nu}]_{\mathrm{eff}} /[\mathrm{El}]_{0} \quad k_{1 \Psi}$

\begin{tabular}{ccccc} 
& $/ \mathrm{mol} \mathrm{L}^{-1}$ & $/ \mathrm{mol} \mathrm{L}^{-1}$ & & $/ \mathrm{s}^{-1}$ \\
\hline fb137.5 & $2.57 \times 10^{-5}$ & $6.94 \times 10^{-4}$ & 27 & $7.21 \times 10^{2}$ \\
fb137.6 & $2.57 \times 10^{-5}$ & $3.47 \times 10^{-4}$ & 14 & $3.21 \times 10^{2}$ \\
fb137.7 & $2.57 \times 10^{-5}$ & $1.39 \times 10^{-4}$ & 5 & $1.37 \times 10^{2}$ \\
\hline
\end{tabular}

$k_{2, \mathrm{~N}}=1.06 \times 10^{6} \mathrm{M}^{-1} \mathrm{~s}^{-1}$

$k_{2, \mathrm{OH}^{-}}=1060 \mathrm{M}^{-1} \mathrm{~s}^{-1}$

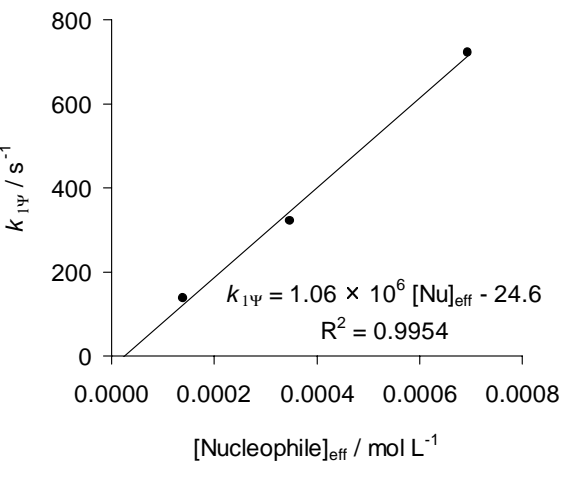

$\mathrm{p} K_{\mathrm{B}}=8.93$ 
19.3.2. Reaction of $\mathrm{p}$-Toluidine (1q) with $(\mathrm{dma})_{2} \mathrm{CH}^{+} \mathrm{BF}_{4}^{-}$(at $20^{\circ} \mathrm{C}$, cosolvent: 1 vol- $\% \mathrm{CH}_{3} \mathrm{CN}$, stopped-flow, detection at $604 \mathrm{~nm}$, $\left.[\mathrm{Nu}]_{\text {eff }}=[\mathrm{Nu}]_{0}, k_{\mathrm{obs}}=k_{1 \Psi}\right)$

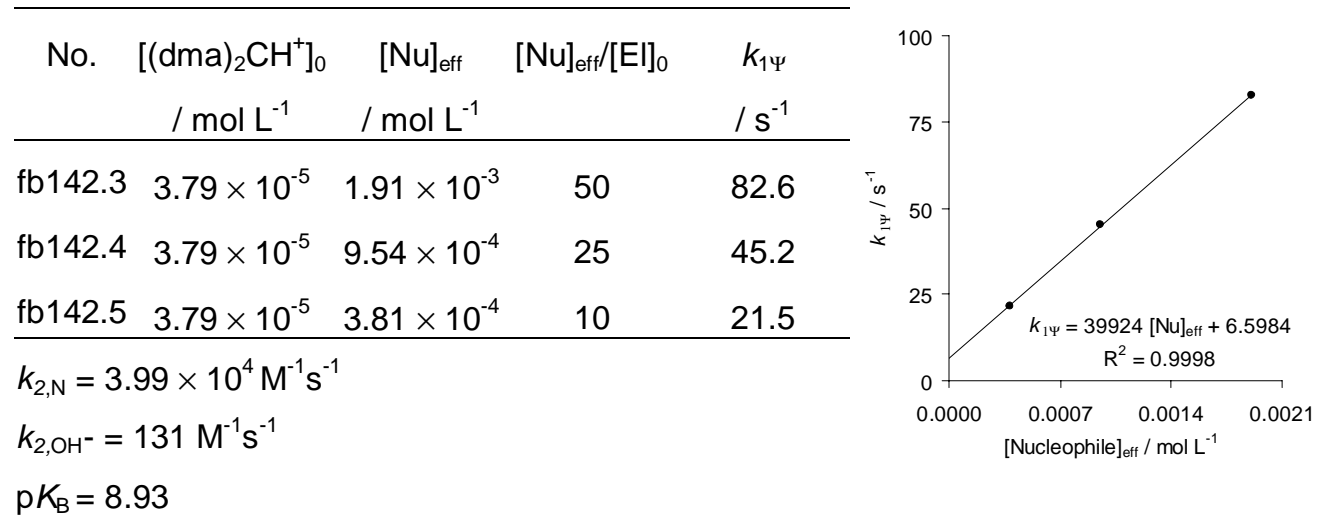

19.3.3. Reaction of p-Toluidine (1q) with (pyr) ${ }_{2} \mathrm{CH}^{+} \mathrm{BF}_{4}^{-}$(at $20^{\circ} \mathrm{C}$, cosolvent: 0.3 vol- $\% \mathrm{CH}_{3} \mathrm{CN}$, stopped-flow, detection at $\left.611 \mathrm{~nm},[\mathrm{Nu}]_{\text {eff }}=[\mathrm{Nu}]_{0}, k_{\mathrm{obs}}=k_{1 \Psi}\right)$

\begin{tabular}{ccccc}
\hline No. & $\begin{array}{c}{\left[(\mathrm{pyr})_{2} \mathrm{CH}^{+}\right]_{0}} \\
/ \mathrm{mol} \mathrm{L}^{-1}\end{array}$ & $\begin{array}{c}{[\mathrm{Nu}]_{\mathrm{eff}}} \\
/ \mathrm{mol} \mathrm{L}^{-1}\end{array}$ & {$[\mathrm{Nu}]_{\mathrm{eff}} /[\mathrm{El}]_{0}$} & $\begin{array}{c}k_{1 \Psi} \\
/ \mathrm{s}^{-1}\end{array}$ \\
\hline fb138.1 & $1.90 \times 10^{-5}$ & $1.39 \times 10^{-2}$ & 731 & $1.48 \times 10^{2}$ \\
fb138.2 & $1.90 \times 10^{-5}$ & $6.95 \times 10^{-3}$ & 366 & 76.5 \\
fb138.3 & $1.90 \times 10^{-5}$ & $3.48 \times 10^{-3}$ & 183 & 40.4 \\
fb138.4 & $1.90 \times 10^{-5}$ & $1.74 \times 10^{-3}$ & 92 & 19.3 \\
fb138.5 & $1.90 \times 10^{-5}$ & $6.94 \times 10^{-4}$ & 37 & 7.24 \\
fb138.6 & $1.90 \times 10^{-5}$ & $3.47 \times 10^{-4}$ & 18 & 3.80 \\
\hline
\end{tabular}

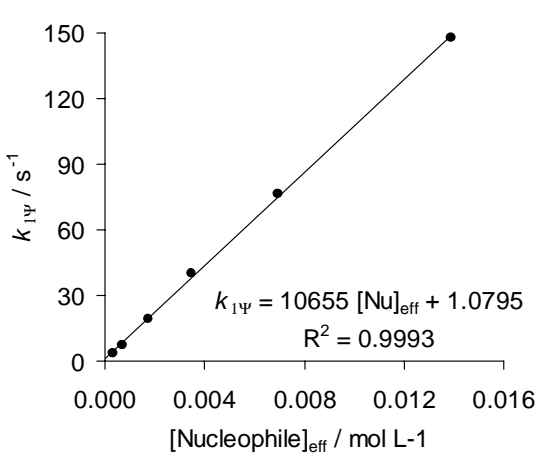

$k_{2, \mathrm{~N}}=1.07 \times 10^{4} \mathrm{M}^{-1} \mathrm{~s}^{-1}$

$k_{2, \mathrm{OH}^{-}}=48.5 \mathrm{M}^{-1} \mathrm{~s}^{-1}$

$\mathrm{p} K_{\mathrm{B}}=8.93$

19.3.4. Reaction of $p$-Toluidine (1q) with (thq) ${ }_{2} \mathrm{CH}^{+} \mathrm{BF}_{4}^{-}$(at $20^{\circ} \mathrm{C}$, cosolvent: 0.4 vol-\% $\mathrm{CH}_{3} \mathrm{CN}$, stopped-flow, detection at $\left.611 \mathrm{~nm},[\mathrm{Nu}]_{\mathrm{eff}}=[\mathrm{Nu}]_{0}, k_{\mathrm{obs}}=k_{1 \Psi}\right)$

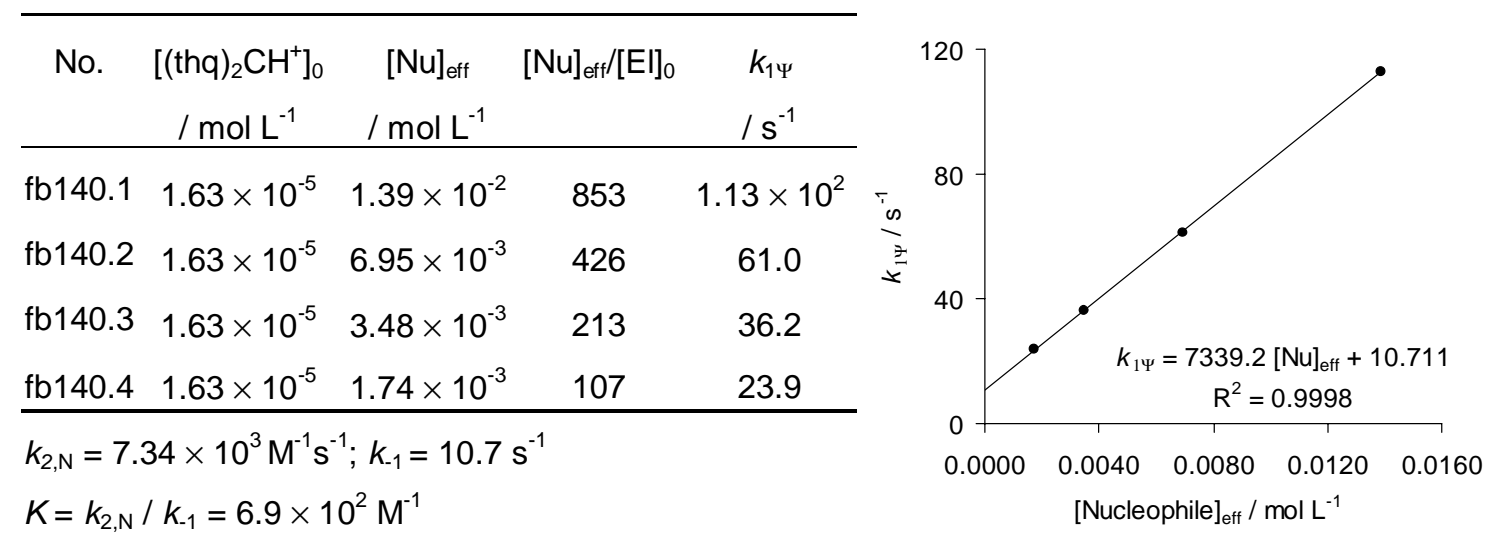


$k_{2, \mathrm{OH}^{-}}=23.6 \mathrm{M}^{-1} \mathrm{~s}^{-1}$

$\mathrm{p} K_{\mathrm{B}}=8.93$

19.3.5. Reaction of $p$-Toluidine (1q) with (ind) ${ }_{2} \mathrm{CH}^{+} \mathrm{BF}_{4}{ }^{-}$(at $20^{\circ} \mathrm{C}$, cosolvent: 0.4 vol- $\% \mathrm{CH}_{3} \mathrm{CN}$, stopped-flow, detection at $\left.611 \mathrm{~nm},[\mathrm{Nu}]_{\mathrm{eff}}=[\mathrm{Nu}]_{0}, k_{\mathrm{obs}}=k_{1 \Psi}\right)$

\begin{tabular}{|c|c|c|c|c|}
\hline No. & $\begin{array}{c}{\left[(\text { ind })_{2} \mathrm{CH}^{+}\right]_{0}} \\
/ \mathrm{mol} \mathrm{L}^{-1}\end{array}$ & $\begin{array}{l}{[\mathrm{Nu}]_{\mathrm{eff}}[\mathrm{N}} \\
/ \mathrm{mol} \mathrm{L}^{-1}\end{array}$ & {$[\mathrm{Nu}]_{\mathrm{eff}} /[\mathrm{El}]_{0}$} & $\begin{array}{l}k_{1 \Psi} \\
/ s^{-1} \\
\end{array}$ \\
\hline fb139.1 & $1.90 \times 10^{-5}$ & $1.39 \times 10^{-2}$ & 732 & 43.2 \\
\hline $\mathrm{fb} 139.2$ & $1.90 \times 10^{-5}$ & $6.95 \times 10^{-3}$ & 366 & 23.5 \\
\hline fb139.3 & $1.90 \times 10^{-5}$ & $3.48 \times 10^{-3}$ & 183 & 12.9 \\
\hline fb139.4 & $1.90 \times 10^{-5}$ & $1.74 \times 10^{-3}$ & 92 & 7.92 \\
\hline fb139.5 & $1.90 \times 10^{-5}$ & $6.94 \times 10^{-4}$ & 37 & 5.58 \\
\hline \multicolumn{5}{|c|}{$k_{2, \mathrm{~N}}=2.88 \times 10^{3} \mathrm{M}^{-1} \mathrm{~s}^{-1} ; k_{-1}=3.20 \mathrm{~s}^{-1}$} \\
\hline \multicolumn{5}{|c|}{$K=k_{2, N} / k_{-1}=9.0 \times 10^{2} \mathrm{M}^{-1}$} \\
\hline \multicolumn{5}{|c|}{$k_{2, \mathrm{OH}^{-}}=10.8 \mathrm{M}^{-1} \mathrm{~s}^{-1}$} \\
\hline \multicolumn{5}{|l|}{$\mathrm{p} K_{\mathrm{B}}=8.93$} \\
\hline 19.4. & Reactivity par & ameters in wat & rater: $N=13$ & $0 ; s=0.79$ \\
\hline \multicolumn{2}{|c|}{ Reference electrophile } & E parameter & \multicolumn{2}{|c|}{$k_{2, \mathrm{~N}}\left(20^{\circ} \mathrm{C}\right) / \mathrm{M}^{-1} \mathrm{~s}^{-1}$} \\
\hline \multicolumn{2}{|c|}{$(\text { mor })_{2} \mathrm{CH}^{+}$} & -5.53 & \multicolumn{2}{|c|}{$1.06 \times 10^{6}$} \\
\hline \multicolumn{2}{|c|}{$(\mathrm{dma})_{2} \mathrm{CH}^{+}$} & -7.02 & \multicolumn{2}{|c|}{$3.99 \times 10^{4}$} \\
\hline \multicolumn{2}{|c|}{$(\text { pyr })_{2} \mathrm{CH}^{+}$} & -7.69 & \multicolumn{2}{|c|}{$1.07 \times 10^{4}$} \\
\hline \multicolumn{2}{|c|}{ (thq) ${ }_{2} \mathrm{CH}^{+}$} & -8.22 & \multicolumn{2}{|c|}{$7.34 \times 10^{3}$} \\
\hline \multicolumn{2}{|c|}{ (ind) ${ }_{2} \mathrm{CH}^{+}$} & -8.76 & \multicolumn{2}{|c|}{$2.88 \times 10^{3}$} \\
\hline
\end{tabular}

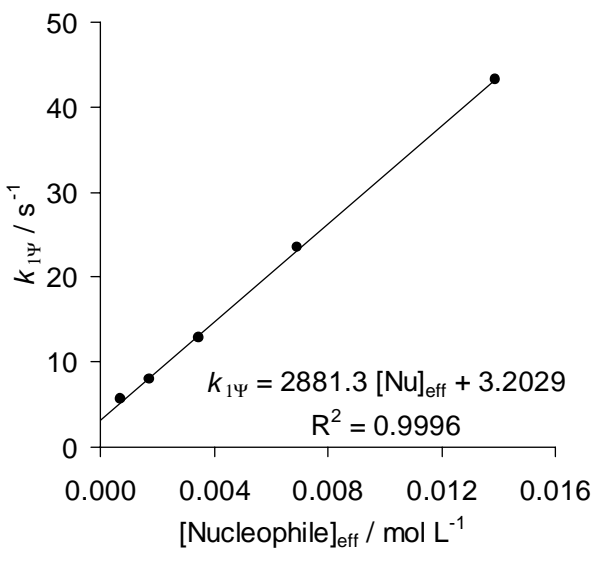




\section{0. p-Anisidine (1r)}

20.1. Rate constants in Acetonitrile

20.1.1. Reaction of p-Anisidine (1r) with (mor) $)_{2} \mathrm{CH}^{+} \mathrm{BF}_{4}{ }^{-}$(at $20^{\circ} \mathrm{C}$, stopped-flow, detection at $620 \mathrm{~nm}$ )

No. $\left[(\mathrm{mor})_{2} \mathrm{CH}^{+}\right]_{0} \quad[\mathrm{Nu}]_{0} \quad[\mathrm{Nu}]_{0} /[\mathrm{El}]_{0} \quad k_{1 \Psi}$

\begin{tabular}{ccccc} 
& $/ \mathrm{mol} \mathrm{L}^{-1}$ & $/ \mathrm{mol} \mathrm{L}^{-1}$ & & $/ \mathrm{s}^{-1}$ \\
\hline j024.4 & $3.42 \times 10^{-5}$ & $2.14 \times 10^{-3}$ & 63 & $1.40 \times 10^{3}$ \\
j024.5 & $3.42 \times 10^{-5}$ & $1.07 \times 10^{-3}$ & 31 & $8.63 \times 10^{2}$ \\
j024.6 & $3.42 \times 10^{-5}$ & $5.35 \times 10^{-4}$ & 16 & $3.67 \times 10^{2}$ \\
\hline
\end{tabular}

$k_{2, \mathrm{~N}}=6.23 \times 10^{5} \mathrm{M}^{-1} \mathrm{~s}^{-1}$

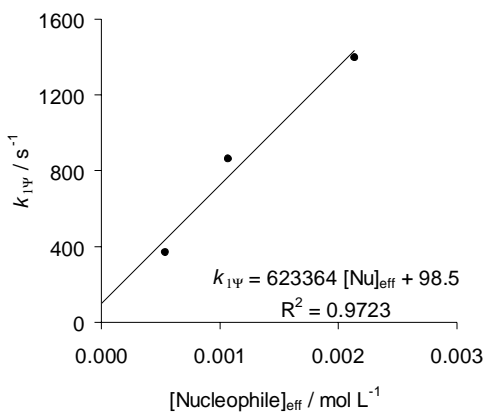

20.1.2. Reaction of $\mathrm{p}$-Anisidine (1r) with $(\mathrm{dma})_{2} \mathrm{CH}^{+} \mathrm{BF}_{4}{ }^{-}$(at $20^{\circ} \mathrm{C}$, stopped-flow, detection at 620 $\mathrm{nm})$

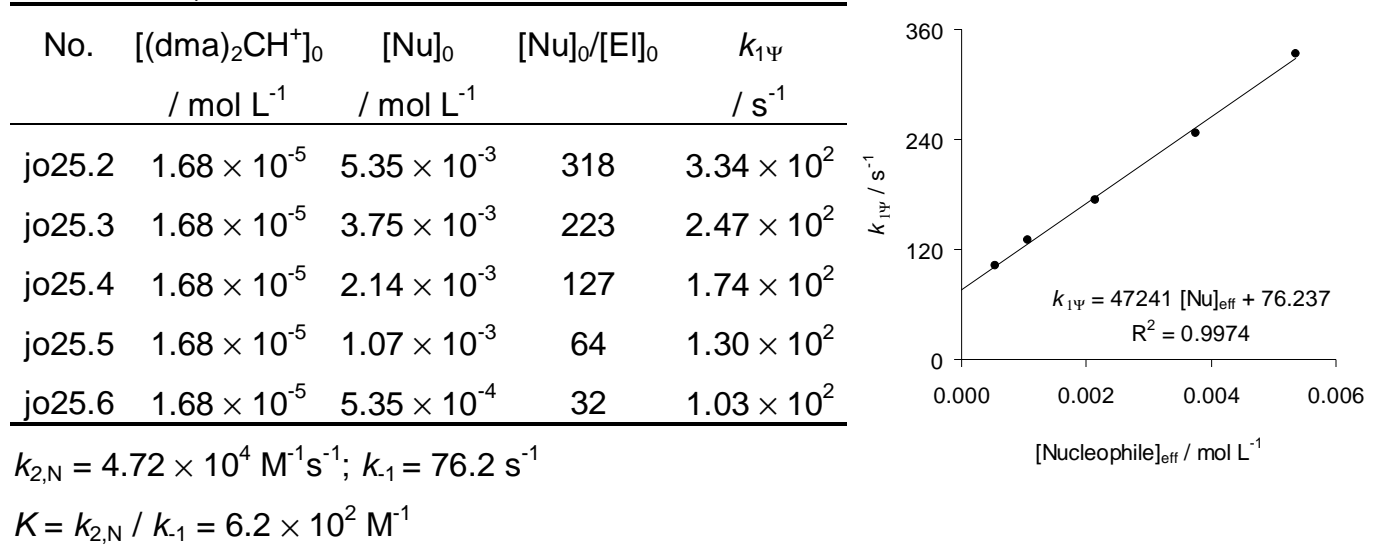

20.1.3. Reaction of p-Anisidine (1r) with (pyr) $)_{2} \mathrm{CH}^{+} \mathrm{BF}_{4}{ }^{-}$(at $20^{\circ} \mathrm{C}$, stopped-flow, detection at $620 \mathrm{~nm}$ )

\begin{tabular}{ccccc} 
No. & $\begin{array}{c}{\left[(\mathrm{pyr})_{2} \mathrm{CH}^{+}\right]_{0}} \\
/ \mathrm{mol} \mathrm{L}^{-1}\end{array}$ & $\begin{array}{c}{[\mathrm{Nu}]_{0}} \\
/ \mathrm{mol} \mathrm{L}^{-1}\end{array}$ & {$[\mathrm{Nu}]_{0} /[\mathrm{El}]_{0}$} & $\begin{array}{c}k_{1 \Psi} \\
/ \mathrm{s}^{-1}\end{array}$ \\
\hline jo26.1 & $2.17 \times 10^{-5}$ & $1.07 \times 10^{-2}$ & 493 & $2.64 \times 10^{2}$ \\
j026.2 & $2.17 \times 10^{-5}$ & $5.35 \times 10^{-3}$ & 247 & $1.80 \times 10^{2}$ \\
j026.3 & $2.17 \times 10^{-5}$ & $3.75 \times 10^{-3}$ & 173 & $1.41 \times 10^{2}$ \\
j026.4 & $2.17 \times 10^{-5}$ & $2.14 \times 10^{-3}$ & 99 & $1.26 \times 10^{2}$ \\
jo26.5 & $2.17 \times 10^{-5}$ & $1.07 \times 10^{-3}$ & 49 & $1.06 \times 10^{2}$ \\
\hline
\end{tabular}

$k_{2, \mathrm{~N}}=1.65 \times 10^{4} \mathrm{M}^{-1} \mathrm{~s}^{-1}$

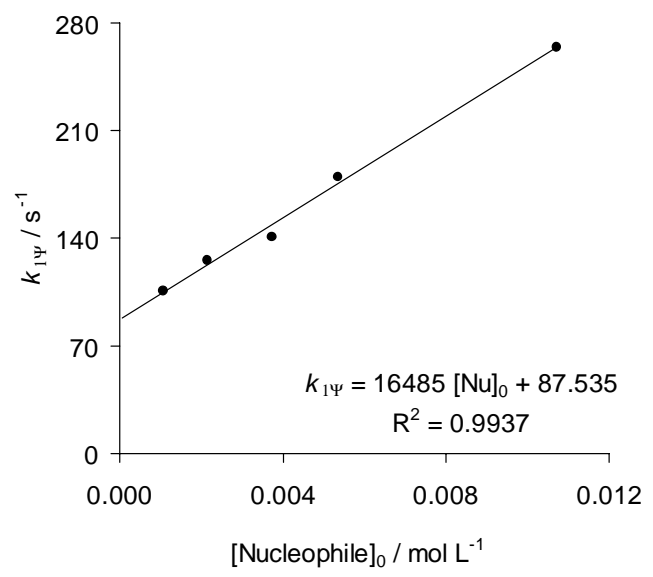


20.2. Reactivity parameters in Acetonitrile: $N=13.42 ; s=0.73$

\begin{tabular}{ccc}
\hline Reference electrophile & $E$ parameter & $k_{2, \mathrm{~N}}\left(20^{\circ} \mathrm{C}\right) / \mathrm{M}^{-1} \mathrm{~s}^{-1}$ \\
\hline$(\mathrm{mor})_{2} \mathrm{CH}^{+}$ & -5.53 & $6.23 \times 10^{5}$ \\
$(\mathrm{dma})_{2} \mathrm{CH}^{+}$ & -7.02 & $4.72 \times 10^{4}$ \\
$(\mathrm{pyr})_{2} \mathrm{CH}^{+}$ & -7.69 & $1.65 \times 10^{4}$ \\
\hline
\end{tabular}

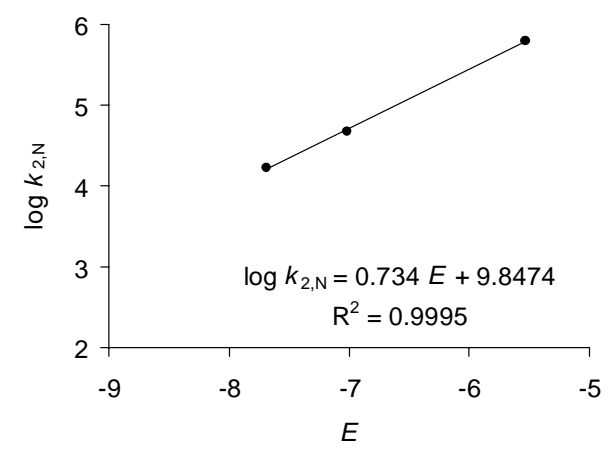

20.3. Rate constants in water

20.3.1. Reaction of p-Anisidine (1r) with (dma) ${ }_{2} \mathrm{CH}^{+} \mathrm{BF}_{4}^{-}$(at $20^{\circ} \mathrm{C}$, cosolvent: 9 vol-\% $\mathrm{CH}_{3} \mathrm{CN}$, stopped-flow, detection at $\left.610 \mathrm{~nm},[\mathrm{Nu}]_{\mathrm{eff}}=[\mathrm{Nu}]_{0}, k_{\mathrm{obs}}=k_{1 \Psi}\right)$

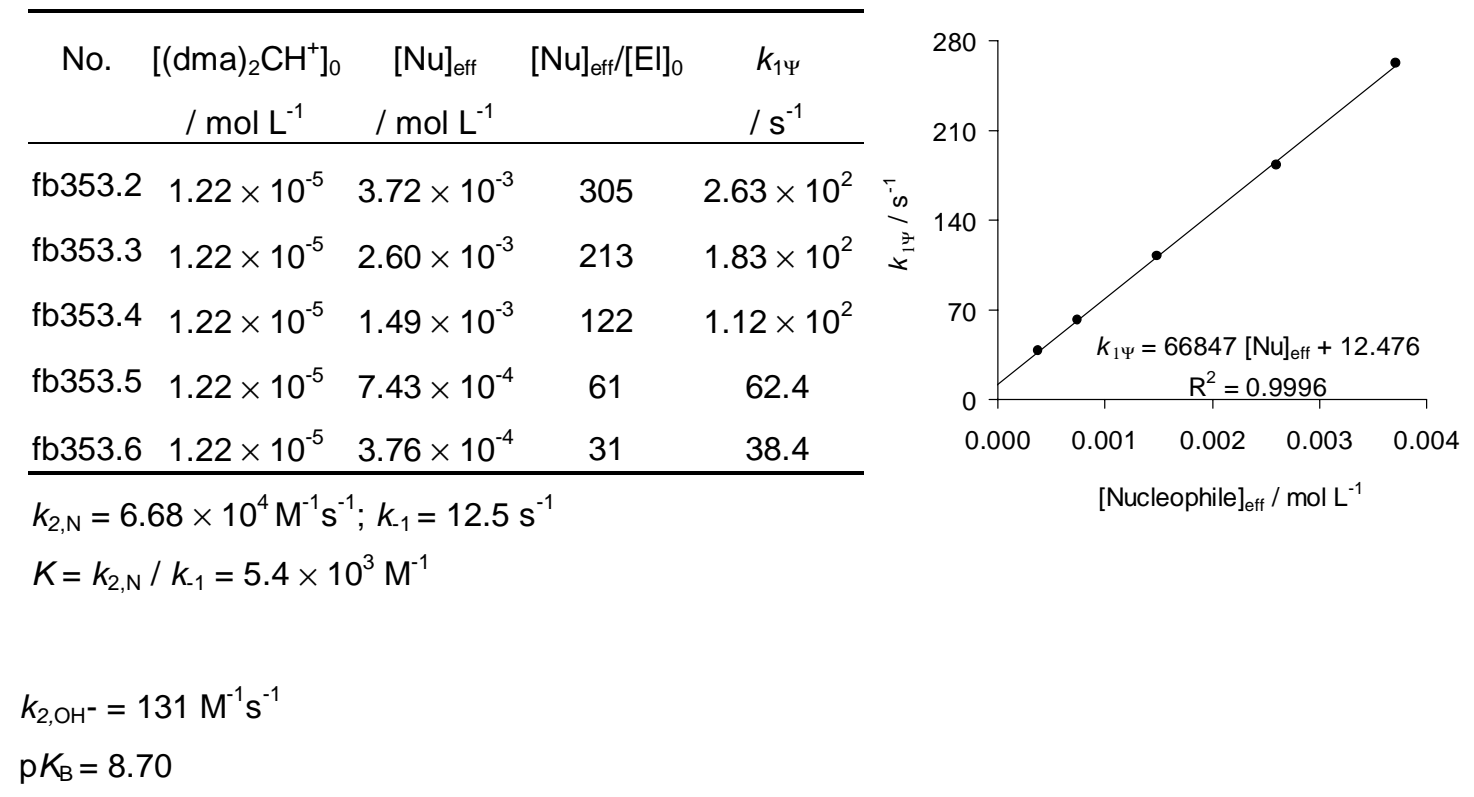


20.3.2. Reaction of $p$-Anisidine (1r) with (thq) ${ }_{2} \mathrm{CH}^{+} \mathrm{BF}_{4}^{-}$(at $20{ }^{\circ} \mathrm{C}$, cosolvent: 0.4 vol-\% $\mathrm{CH}_{3} \mathrm{CN}$, stopped-flow, detection at $620 \mathrm{~nm}$, $\left.[\mathrm{Nu}]_{\mathrm{eff}}=[\mathrm{Nu}]_{0}, k_{\mathrm{obs}}=k_{1 \Psi}\right)$

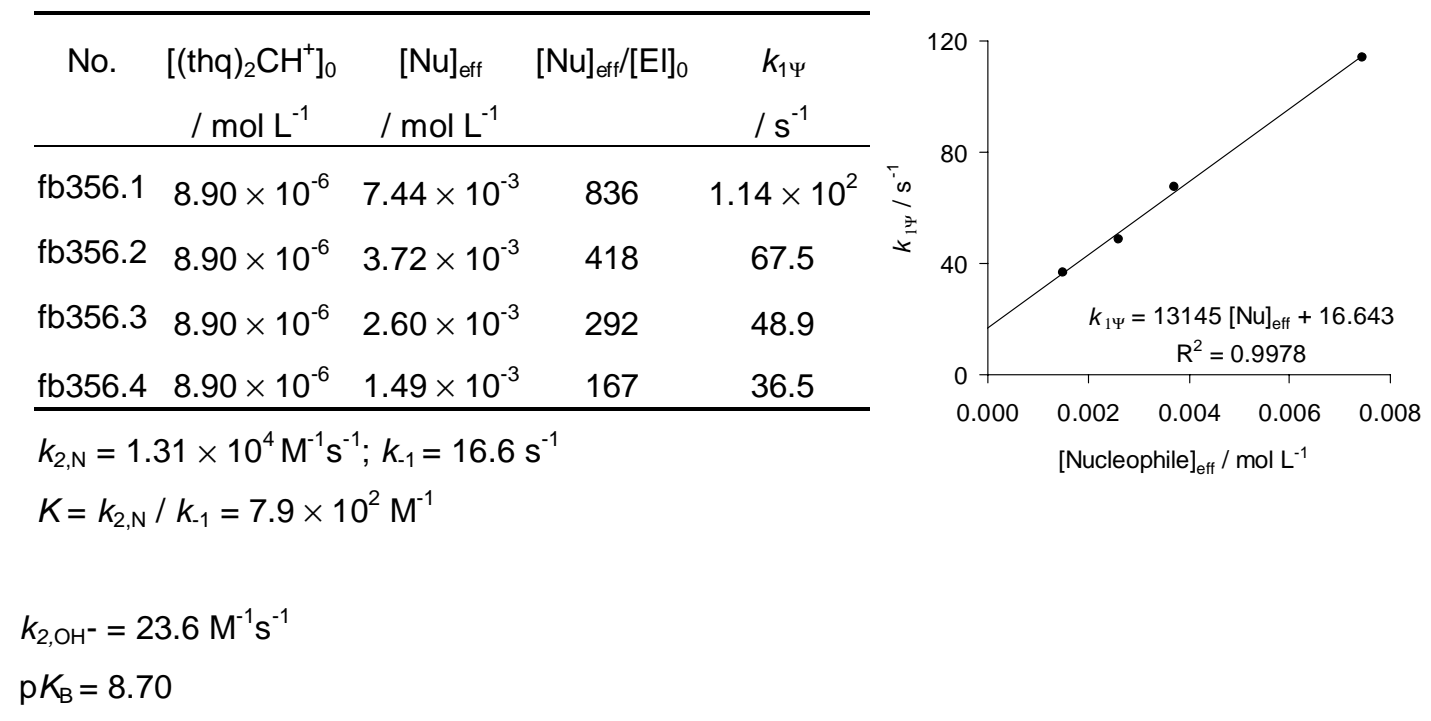

20.3.3. Reaction of $\mathrm{p}$-Anisidine (1r) with (lil) ${ }_{2} \mathrm{CH}^{+} \mathrm{BF}_{4}^{-}$(at $20{ }^{\circ} \mathrm{C}$, cosolvent: 0.2 vol-\% $\mathrm{CH}_{3} \mathrm{CN}$, stopped-flow, detection at $\left.634 \mathrm{~nm},[\mathrm{Nu}]_{\mathrm{eff}}=[\mathrm{Nu}]_{0}, k_{\mathrm{obs}}=k_{1 \Psi}\right)$

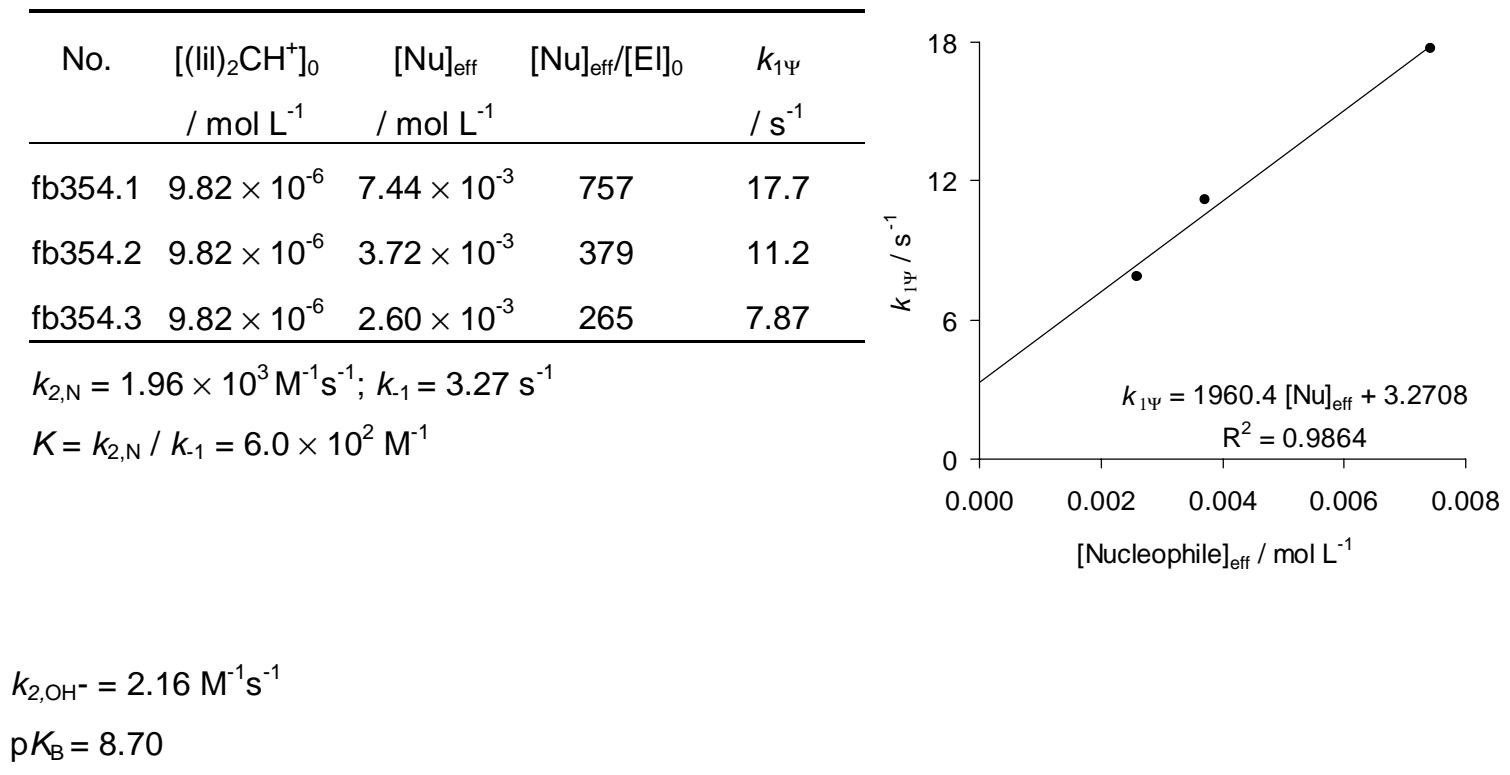


20.4. Reactivity parameters in water: $N=16.53 ; s=0.50$

\begin{tabular}{ccc}
\hline Reference electrophile & $E$ parameter & $k_{2, \mathrm{~N}}\left(20^{\circ} \mathrm{C}\right) / \mathrm{M}^{-1} \mathrm{~s}^{-1}$ \\
\hline$(\mathrm{dma})_{2} \mathrm{CH}^{+}$ & -7.02 & $6.68 \times 10^{4}$ \\
$(\text { thq })_{2} \mathrm{CH}^{+}$ & -8.22 & $1.31 \times 10^{4}$ \\
$(\mathrm{lil})_{2} \mathrm{CH}^{+}$ & -10.04 & $1.96 \times 10^{3}$ \\
\hline
\end{tabular}

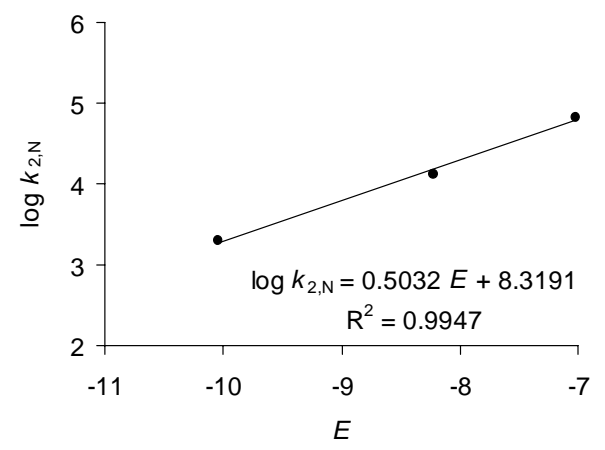

\section{Dimethylamine (1s)}

21.1. Rate constants in water

21.1.1. Reaction of Dimethylamine (1s) with (mor) $)_{2} \mathrm{CH}^{+} \mathrm{BF}_{4}^{-}$(at $20^{\circ} \mathrm{C}$, cosolvent 9 vol- $\% \mathrm{CH}_{3} \mathrm{CN}$, stopped-flow, detection at $610 \mathrm{~nm}$ )

\begin{tabular}{ccccccccc}
\hline No. & $\begin{array}{c}{\left[(\mathrm{mor})_{2} \mathrm{CH}^{+}\right]_{0}} \\
/ \mathrm{mol} \mathrm{L}^{-1}\end{array}$ & $\begin{array}{c}{[\mathrm{Nu}]_{0}} \\
/ \mathrm{mol} \mathrm{L}^{-1}\end{array}$ & $\begin{array}{c}{[\mathrm{Nu}]_{\mathrm{eff}}} \\
/ \mathrm{mol} \mathrm{L}^{-1}\end{array}$ & $\begin{array}{c}{[\mathrm{OH}]} \\
/ \mathrm{mol} \mathrm{L}^{-1}\end{array}$ & $\begin{array}{c}{[\mathrm{Nu}]_{\mathrm{eff}} /[\mathrm{El}]_{0}} \\
k_{\mathrm{obs}}\end{array}$ & $\begin{array}{c}k_{1 \Psi, \mathrm{OH}^{-}} \\
/ \mathrm{s}^{-1}\end{array}$ & $\begin{array}{c}k_{1 \Psi} \\
/ \mathrm{s}^{-1}\end{array}$ & $/ \mathrm{s}^{-1}$ \\
\hline fb198.4 & $1.50 \times 10^{-5}$ & $8.94 \times 10^{-4}$ & $4.02 \times 10^{-4}$ & $4.92 \times 10^{-4}$ & 27 & $2.59 \times 10^{2}$ & 0.522 & $2.58 \times 10^{2}$ \\
fb198.5 & $1.50 \times 10^{-5}$ & $4.47 \times 10^{-4}$ & $1.48 \times 10^{-4}$ & $2.99 \times 10^{-4}$ & 10 & $1.04 \times 10^{2}$ & 0.317 & $1.04 \times 10^{2}$ \\
\hline
\end{tabular}

$$
\begin{aligned}
& k_{2, \mathrm{~N}}=6.10 \times 10^{5} \mathrm{M}^{-1} \mathrm{~s}^{-1} \\
& k_{2, \mathrm{OH}^{-}}=1060 \mathrm{M}^{-1} \mathrm{~s}^{-1} \\
& \mathrm{p} K_{\mathrm{B}}=3.22
\end{aligned}
$$

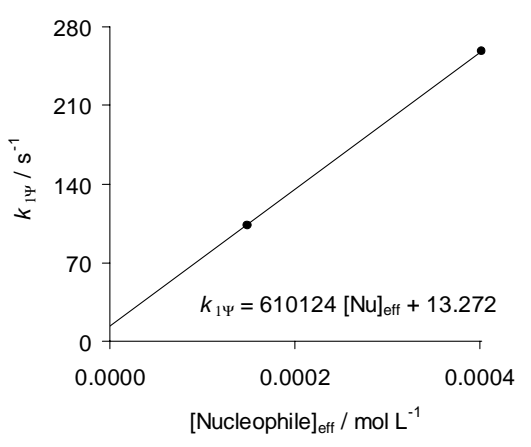

\begin{tabular}{|c|c|c|c|c|c|c|c|c|}
\hline No. & $\begin{array}{c}{\left[(\mathrm{dma})_{2} \mathrm{CH}^{+}\right]_{0}} \\
/ \mathrm{mol} \mathrm{L}^{-1}\end{array}$ & $\begin{array}{c}{[\mathrm{Nu}]_{0}} \\
/ \mathrm{mol} \mathrm{L}^{-1}\end{array}$ & $\begin{array}{c}{[\mathrm{Nu}]_{\mathrm{eff}}} \\
/ \mathrm{mol} \mathrm{L}^{-1}\end{array}$ & $\begin{array}{c}{\left[\mathrm{OH}^{-}\right]} \\
/ \mathrm{mol} \mathrm{L}^{-1}\end{array}$ & {$[\mathrm{Nu}]_{\mathrm{eff}} /[\mathrm{El}]_{0}$} & $\begin{array}{l}k_{\mathrm{obs}} \\
/ \mathrm{s}^{-1}\end{array}$ & $\begin{array}{c}k_{1 \Psi, \mathrm{OH}^{-}} \\
/ \mathrm{s}^{-1}\end{array}$ & $\begin{array}{l}k_{1 \Psi} \\
/ \mathrm{s}^{-1}\end{array}$ \\
\hline ccy124.1 & $5.95 \times 10^{-5}$ & $5.45 \times 10^{-3}$ & $3.91 \times 10^{-3}$ & $1.54 \times 10^{-3}$ & 66 & 407 & 0.201 & 407 \\
\hline cсу124.2 & $5.95 \times 10^{-5}$ & $2.73 \times 10^{-3}$ & $1.71 \times 10^{-3}$ & $1.02 \times 10^{-3}$ & 29 & 174 & 0.133 & 174 \\
\hline ccy124.3 & $5.95 \times 10^{-5}$ & $1.91 \times 10^{-3}$ & $1.10 \times 10^{-3}$ & $8.13 \times 10^{-4}$ & 18 & 110 & 0.107 & 110 \\
\hline ccy 124.4 & $5.95 \times 10^{-5}$ & $1.09 \times 10^{-3}$ & $5.27 \times 10^{-4}$ & $5.63 \times 10^{-4}$ & 9 & 52.2 & $7.38 \times 10^{-2}$ & 52.1 \\
\hline cсy124.5 & $5.95 \times 10^{-5}$ & $5.45 \times 10^{-4}$ & $1.99 \times 10^{-4}$ & $3.46 \times 10^{-4}$ & 3 & 18.4 & $4.53 \times 10^{-2}$ & 18.4 \\
\hline
\end{tabular}

21.1.2. Reaction of Dimethylamine (1s) with (dma) ${ }_{2} \mathrm{CH}^{+} \mathrm{BF}_{4}^{-}$(at $20^{\circ} \mathrm{C}$, cosolvent 0.5 vol- $\% \mathrm{CH}_{3} \mathrm{CN}$, stopped-flow, detection at $610 \mathrm{~nm})$ 


$$
\begin{aligned}
& k_{2, \mathrm{~N}}=1.05 \times 10^{5} \mathrm{M}^{-1} \mathrm{~s}^{-1} \\
& k_{2, \mathrm{OH}^{-}}=131 \mathrm{M}^{-1} \mathrm{~s}^{-1} \\
& \mathrm{p} K_{\mathrm{B}}=3.22
\end{aligned}
$$

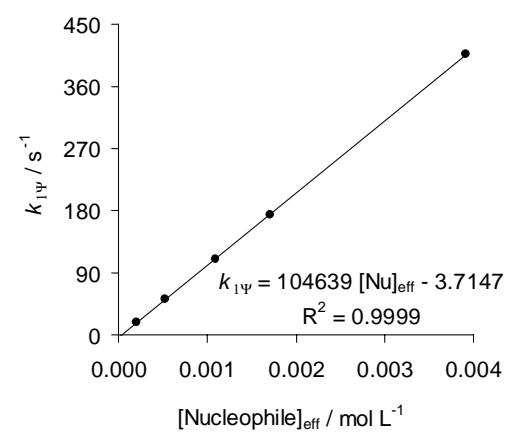

21.1.3. Reaction of Dimethylamine (1s) with $(\mathrm{pyr})_{2} \mathrm{CH}^{+} \mathrm{BF}_{4}^{-}$(at $20^{\circ} \mathrm{C}$, cosolvent 0.2 vol- $\% \mathrm{CH}_{3} \mathrm{CN}$, stopped-flow, detection at $610 \mathrm{~nm}$ )

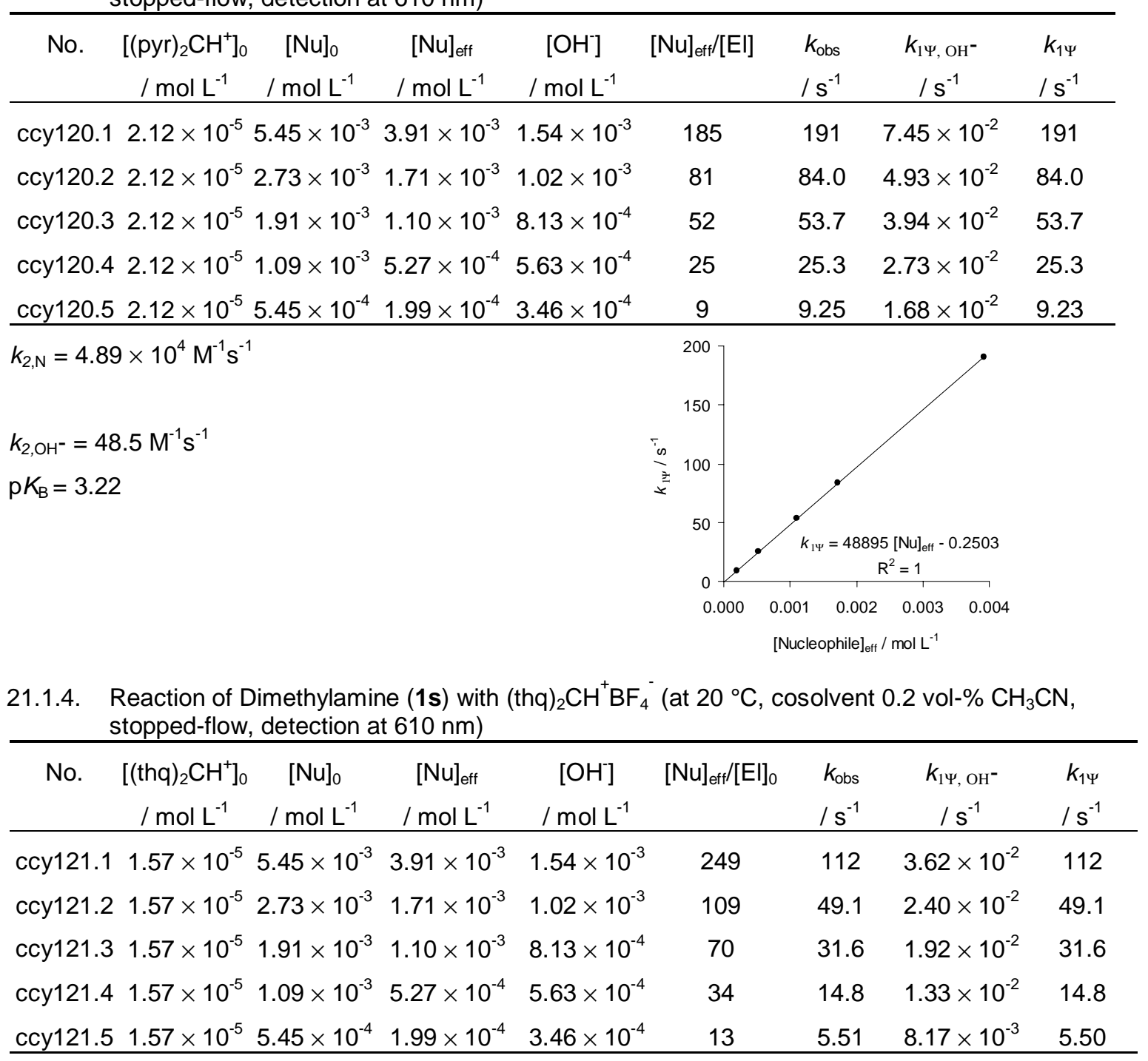




$$
\begin{aligned}
& k_{2, \mathrm{~N}}=2.87 \times 10^{4} \mathrm{M}^{-1} \mathrm{~s}^{-1} \\
& k_{2, \mathrm{OH}^{-}}=23.6 \mathrm{M}^{-1} \mathrm{~s}^{-1} \\
& \mathrm{p} K_{\mathrm{B}}=3.22
\end{aligned}
$$

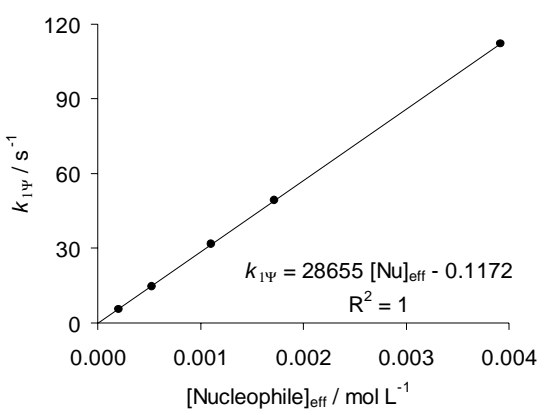

21.1.5. Reaction of Dimethylamine (1s) with (ind) ${ }_{2} \mathrm{CH}^{+} \mathrm{BF}_{4}^{-}$(at $20^{\circ} \mathrm{C}$, cosolvent 0.3 vol- $\% \mathrm{CH}_{3} \mathrm{CN}$, stopped-flow, detection at $610 \mathrm{~nm})$

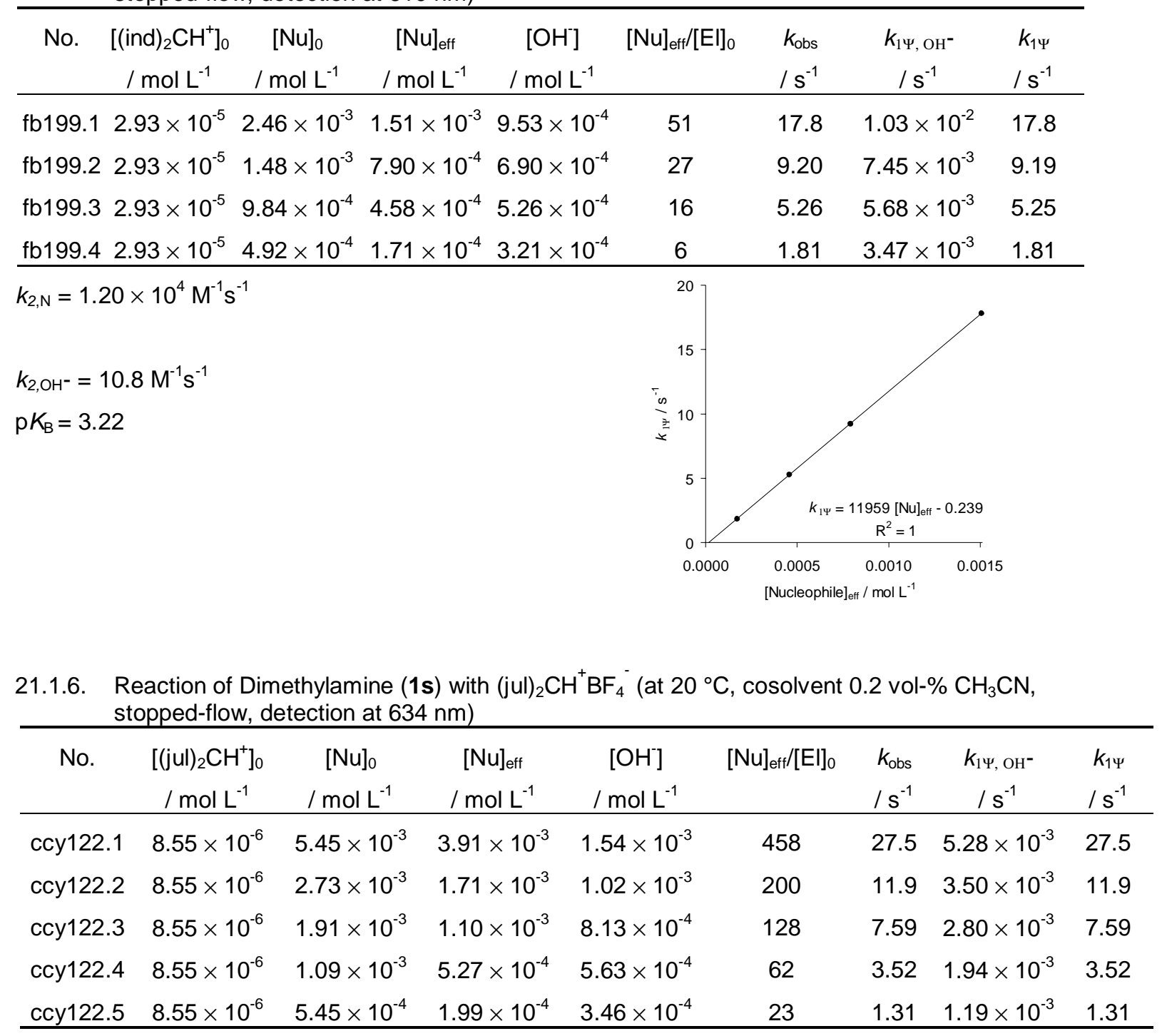




$$
\begin{aligned}
& k_{2, \mathrm{~N}}=7.06 \times 10^{3} \mathrm{M}^{-1} \mathrm{~s}^{-1} \\
& k_{2, \mathrm{OH}^{-}}=3.44 \mathrm{M}^{-1} \mathrm{~s}^{-1} \\
& \mathrm{p} K_{\mathrm{B}}=3.22
\end{aligned}
$$

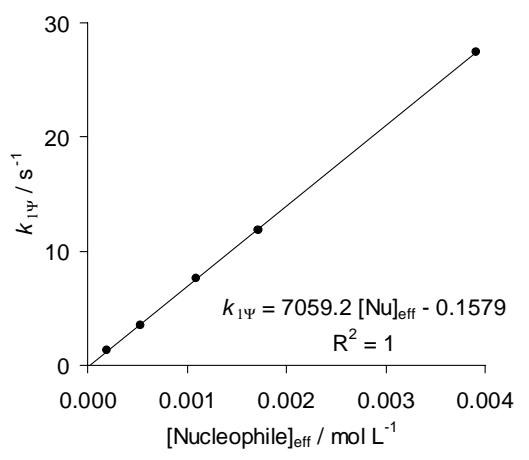

\begin{tabular}{|c|c|c|c|c|c|c|c|c|}
\hline No. & $\begin{array}{c}{\left[(\mathrm{lil})_{2} \mathrm{CH}^{+}\right]_{0}} \\
/ \mathrm{mol} \mathrm{L}^{-1}\end{array}$ & $\begin{array}{c}{[\mathrm{Nu}]_{0}} \\
/ \mathrm{mol} \mathrm{L}^{-1}\end{array}$ & $\begin{array}{c}{[\mathrm{Nu}]_{\mathrm{eff}}} \\
/ \mathrm{mol} \mathrm{L}^{-1}\end{array}$ & $\begin{array}{c}{\left[\mathrm{OH}^{-}\right]} \\
/ \mathrm{mol} \mathrm{L}^{-1}\end{array}$ & {$[\mathrm{Nu}]_{\mathrm{eff}}\left[[\mathrm{El}]_{0}\right.$} & $\begin{array}{l}k_{\mathrm{obs}} \\
/ \mathrm{s}^{-1}\end{array}$ & $\begin{array}{c}k_{1 \Psi, \mathrm{OH}^{-}} \\
/ \mathrm{s}^{-1}\end{array}$ & $\begin{array}{l}k_{1 \Psi} \\
/ \mathrm{s}^{-1}\end{array}$ \\
\hline ccy123.1 & $8.55 \times 10^{-6}$ & $5.45 \times 10^{-3}$ & $3.91 \times 10^{-3}$ & $1.54 \times 10^{-3}$ & 458 & 13.7 & $3.32 \times 10^{-3}$ & 13.7 \\
\hline ccy 123.2 & $8.55 \times 10^{-6}$ & $2.73 \times 10^{-3}$ & $1.71 \times 10^{-3}$ & $1.02 \times 10^{-3}$ & 200 & 5.91 & $2.19 \times 10^{-3}$ & 5.91 \\
\hline ccy123.3 & $8.55 \times 10^{-6}$ & $1.91 \times 10^{-3}$ & $1.10 \times 10^{-3}$ & $8.13 \times 10^{-4}$ & 128 & 3.77 & $1.76 \times 10^{-3}$ & 3.77 \\
\hline ccy 123.4 & $8.55 \times 10^{-6}$ & $1.09 \times 10^{-3}$ & $5.27 \times 10^{-4}$ & $5.63 \times 10^{-4}$ & 62 & 1.75 & $1.22 \times 10^{-3}$ & 1.75 \\
\hline ccy123.5 & $8.55 \times 10^{-6}$ & $5.45 \times 10^{-4}$ & $1.99 \times 10^{-4}$ & $3.46 \times 10^{-4}$ & 23 & 0.649 & $7.48 \times 10^{-4}$ & 0.648 \\
\hline \multicolumn{9}{|c|}{$k_{2, \mathrm{~N}}=3.52 \times 10^{3} \mathrm{M}^{-1} \mathrm{~s}^{-1}$} \\
\hline \multicolumn{9}{|c|}{$k_{2, \mathrm{OH}^{-}}=2.16 \mathrm{M}^{-1} \mathrm{~s}^{-1}$} \\
\hline \multicolumn{9}{|c|}{$\mathrm{p} K_{\mathrm{B}}=3.22$} \\
\hline & & & & & & $D^{2}$ & & \\
\hline & & & & & $\begin{array}{l}0.001 \quad 0.0 \\
\text { [Nucleophile }\end{array}$ & $\begin{array}{l}020.00 \\
\text { eff } / \mathrm{mol} \mathrm{L}^{-1}\end{array}$ & 0.004 & \\
\hline
\end{tabular}

21.1.7. Reaction of Dimethylamine (1s) with (lil) $)_{2} \mathrm{CH}^{+} \mathrm{BF}_{4}^{-}$(at $20^{\circ} \mathrm{C}$, cosolvent 0.2 vol- $\% \mathrm{CH}_{3} \mathrm{CN}$, stopped-flow, detection at $634 \mathrm{~nm}$ )

21.2. Reactivity parameters in water: $N=17.12 ; s=0.50$

\begin{tabular}{ccc}
\hline Reference electrophile & $E$ parameter & $k_{2, \mathrm{~N}}\left(20^{\circ} \mathrm{C}\right) / \mathrm{M}^{-1} \mathrm{~s}^{-1}$ \\
\hline$(\text { mor })_{2} \mathrm{CH}^{+}$ & -5.53 & $6.10 \times 10^{5}$ \\
$(\mathrm{dma})_{2} \mathrm{CH}^{+}$ & -7.02 & $1.05 \times 10^{5}$ \\
$(\text { pyr })_{2} \mathrm{CH}^{+}$ & -7.69 & $4.89 \times 10^{4}$ \\
$(\text { thq })_{2} \mathrm{CH}^{+}$ & -8.22 & $2.87 \times 10^{4}$ \\
$(\text { ind })_{2} \mathrm{CH}^{+}$ & -8.76 & $1.20 \times 10^{4}$ \\
$(\mathrm{jul})_{2} \mathrm{CH}^{+}$ & -9.45 & $7.06 \times 10^{3}$ \\
$(\text { lil })_{2} \mathrm{CH}^{+}$ & -10.04 & $3.52 \times 10^{3}$ \\
\hline
\end{tabular}

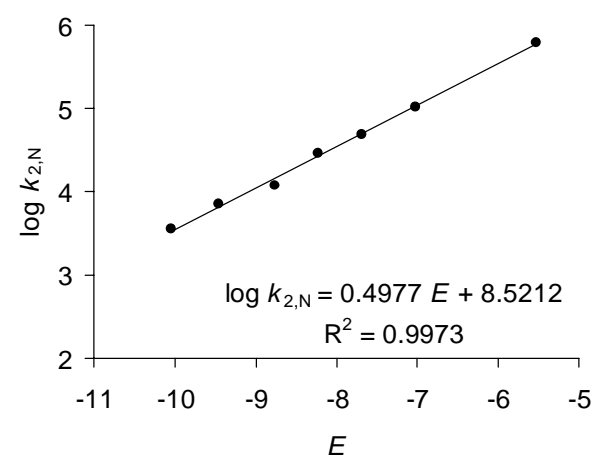




\section{Diethylamine (1t)}

22.1. Rate constants in water

22.1.1. Reaction of Diethylamine (1t) with (mor) ${ }_{2} \mathrm{CH}^{+} \mathrm{BF}_{4}{ }^{-}$(at $20^{\circ} \mathrm{C}$, cosolvent 9 vol- $\% \mathrm{CH}_{3} \mathrm{CN}$, stopped-flow, detection at $610 \mathrm{~nm}$ )

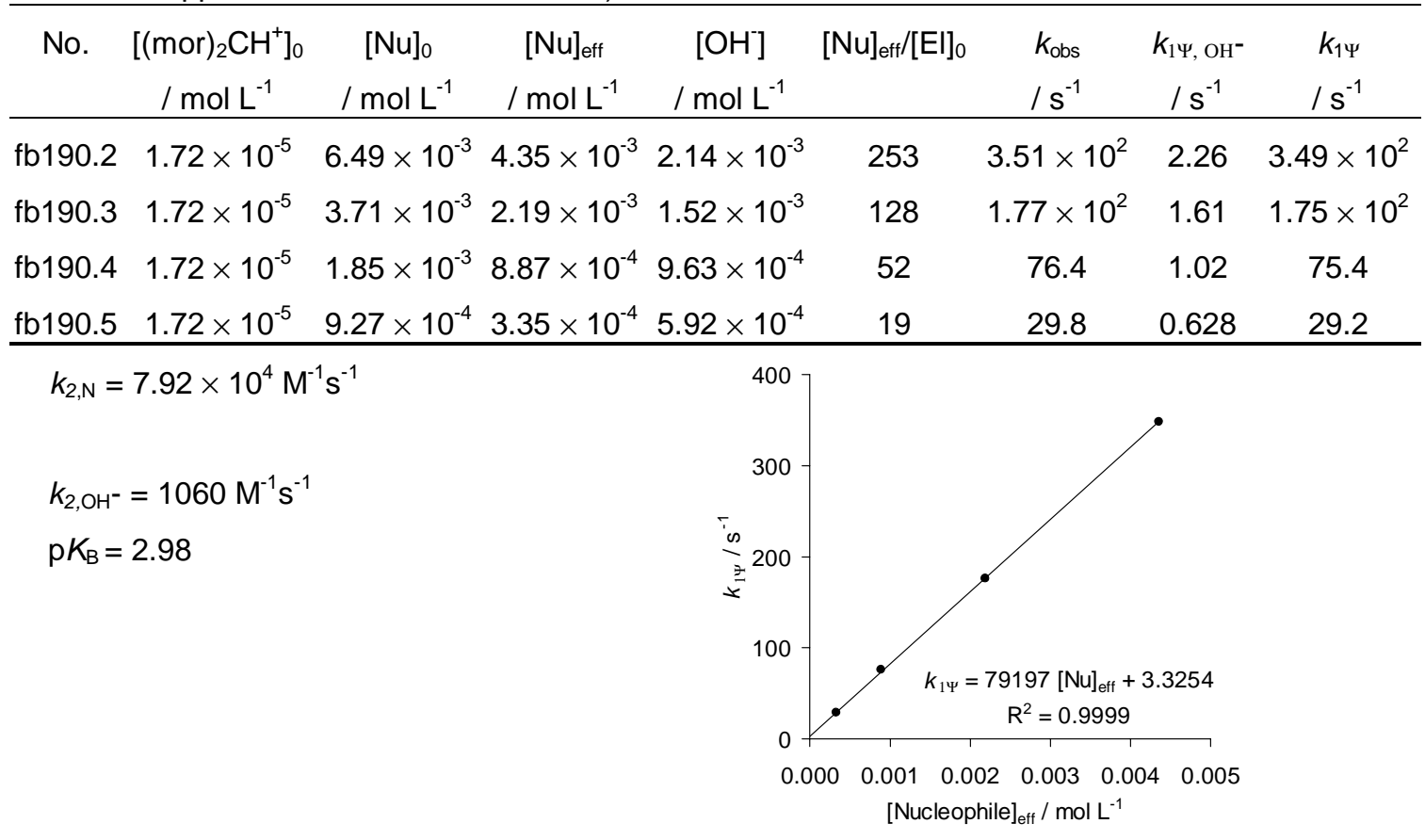

22.1.2. Reaction of Diethylamine (1t) with $(\mathrm{dma})_{2} \mathrm{CH}^{+} \mathrm{BF}_{4}{ }^{-}$(at $20^{\circ} \mathrm{C}$, cosolvent 0.5 vol- $\% \mathrm{CH}_{3} \mathrm{CN}$, stopped-flow, detection at $610 \mathrm{~nm}$ )

\begin{tabular}{|c|c|c|c|c|c|c|c|c|}
\hline No. & $\begin{array}{c}{\left[(\mathrm{dma})_{2} \mathrm{CH}^{+}\right]_{0}} \\
/ \mathrm{mol} \mathrm{L}^{-1}\end{array}$ & $\begin{array}{c}{[\mathrm{Nu}]_{0}} \\
/ \mathrm{mol} \mathrm{L}^{-1}\end{array}$ & $\begin{array}{c}{[\mathrm{Nu}]_{\mathrm{eff}}} \\
/ \mathrm{mol} \mathrm{L}^{-1}\end{array}$ & $\begin{array}{c}{\left[\mathrm{OH}^{-}\right]} \\
/ \mathrm{mol} \mathrm{L}^{-1}\end{array}$ & {$[\mathrm{Nu}]_{\mathrm{efff}} /[\mathrm{El}]_{0}$} & $\begin{array}{l}k_{\mathrm{obs}} \\
/ \mathrm{s}^{-1}\end{array}$ & $\begin{array}{c}k_{1 \Psi, \mathrm{OH}^{-}} \\
/ \mathrm{s}^{-1}\end{array}$ & $\begin{array}{l}k_{1 \Psi} \\
/ s^{-1}\end{array}$ \\
\hline ccy145.1 & $5.95 \times 10^{-5}$ & $1.28 \times 10^{-2}$ & $9.63 \times 10^{-3}$ & $3.17 \times 10^{-3}$ & 162 & 108 & 0.416 & 108 \\
\hline ccy145.2 & $5.95 \times 10^{-5}$ & $6.40 \times 10^{-3}$ & $4.28 \times 10^{-3}$ & $2.12 \times 10^{-3}$ & 72 & 50.1 & 0.277 & 49.8 \\
\hline ccy 145.3 & $5.95 \times 10^{-5}$ & $4.48 \times 10^{-3}$ & $2.78 \times 10^{-3}$ & $1.70 \times 10^{-3}$ & 47 & 32.7 & 0.223 & 32.5 \\
\hline ccy145.4 & $5.95 \times 10^{-5}$ & $2.56 \times 10^{-3}$ & $1.36 \times 10^{-3}$ & $1.20 \times 10^{-3}$ & 23 & 15.7 & 0.157 & 15.5 \\
\hline ccy145.5 & $5.95 \times 10^{-5}$ & $1.28 \times 10^{-3}$ & $5.33 \times 10^{-4}$ & $7.47 \times 10^{-4}$ & 9 & 5.90 & $9.79 \times 10^{-2}$ & 5.80 \\
\hline \multicolumn{3}{|c|}{$k_{2, \mathrm{~N}}=1.12 \times 10^{4} \mathrm{M}^{-1} \mathrm{~s}^{-1}$} & & 120 & & & & \\
\hline \multicolumn{3}{|c|}{$k_{2, \mathrm{OH}^{-}}=131 \mathrm{M}^{-1} \mathrm{~s}^{-1}$} & & & & & & \\
\hline \multicolumn{2}{|c|}{$\mathrm{p} K_{\mathrm{B}}=2.98$} & & & 60 & & & & \\
\hline & & & & & & & & \\
\hline & & & & 0.0000 & $\begin{array}{c}0.0025 \quad 0 . \\
\text { [Nucleophil }\end{array}$ & $\begin{array}{l}050 \\
\text { ]eff } / n\end{array}$ & $\begin{array}{ll}75 & 0.0100\end{array}$ & \\
\hline
\end{tabular}


22.1.3. Reaction of Diethylamine (1t) with (pyr) ${ }_{2} \mathrm{CH}^{+} \mathrm{BF}_{4}^{-}$(at $20^{\circ} \mathrm{C}$, cosolvent 0.1 vol- $\% \mathrm{CH}_{3} \mathrm{CN}$, stopped-flow, detection at $610 \mathrm{~nm}$ )

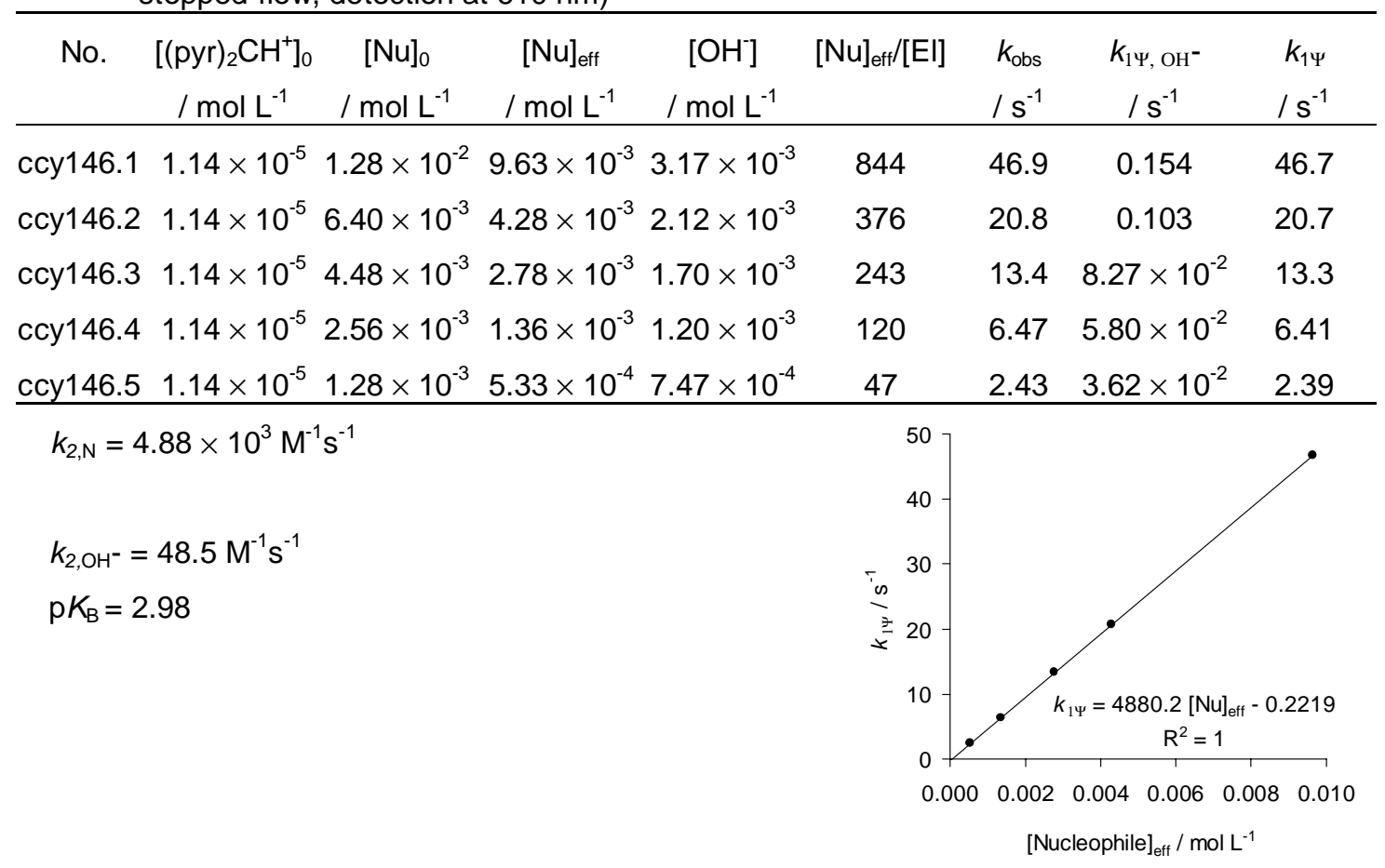

22.1.4. Reaction of Diethylamine (1t) with (thq) ${ }_{2} \mathrm{CH}^{+} \mathrm{BF}_{4}^{-}$(at $20^{\circ} \mathrm{C}$, cosolvent 0.4 vol- $\% \mathrm{CH}_{3} \mathrm{CN}$, stopped-flow, detection at $610 \mathrm{~nm}$ )

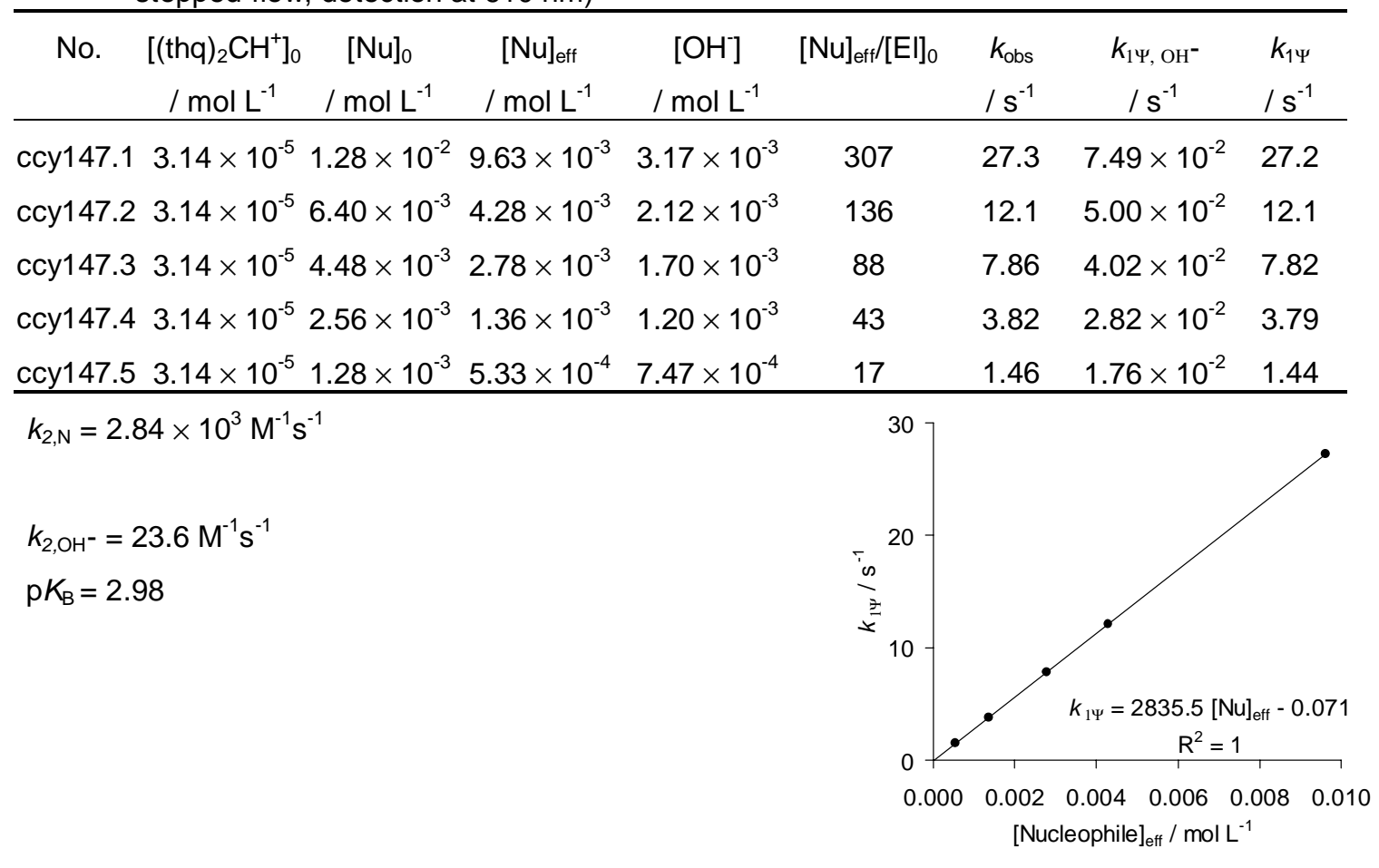


22.1.5. Reaction of Diethylamine (1t) with (ind) ${ }_{2} \mathrm{CH}^{+} \mathrm{BF}_{4}^{-}$(at $20{ }^{\circ} \mathrm{C}$, cosolvent 0.2 vol- $\% \mathrm{CH}_{3} \mathrm{CN}$, stopped-flow, detection at $613 \mathrm{~nm}$ )

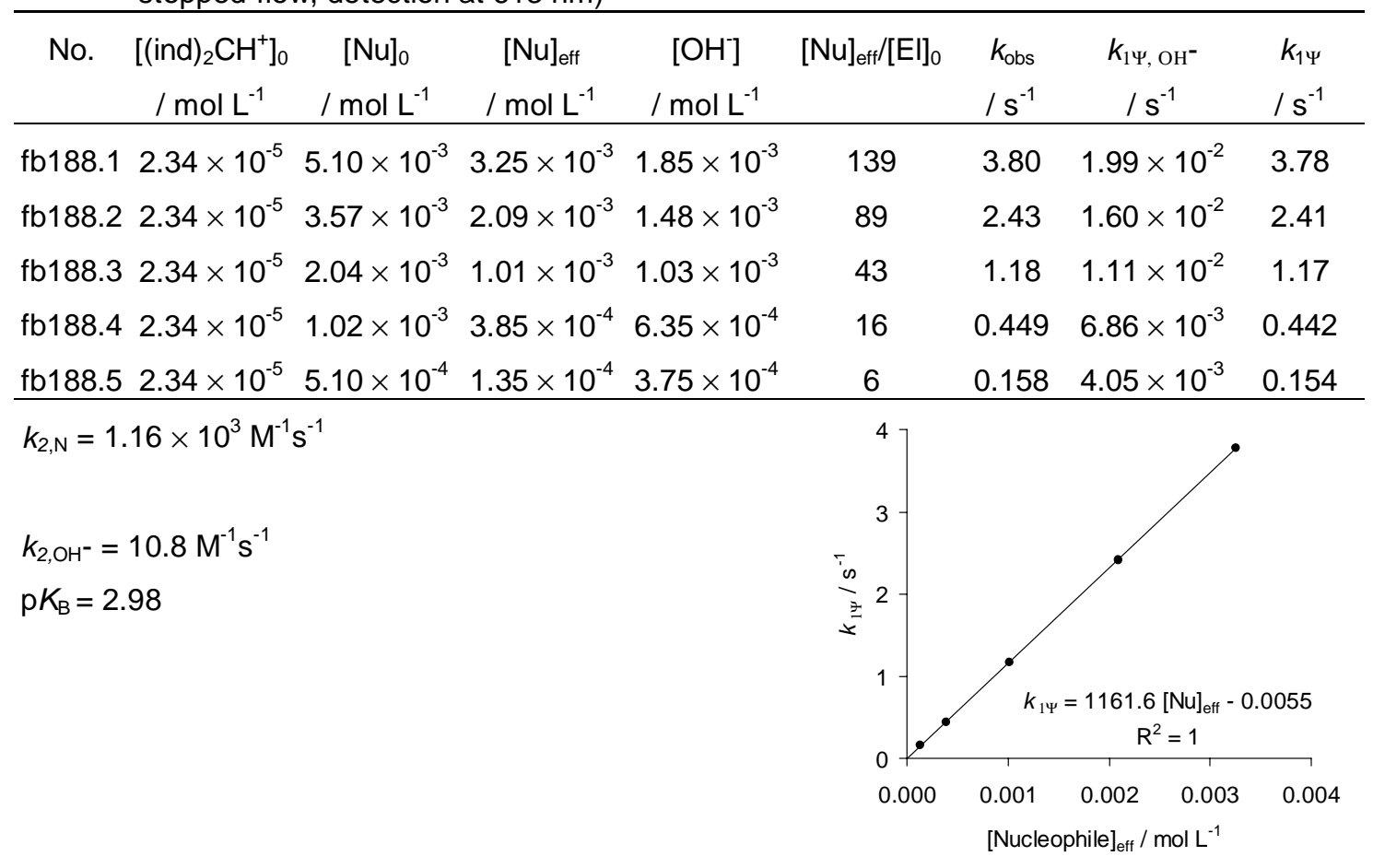

22.1.6. Reaction of Diethylamine (1t) with (jul) ${ }_{2} \mathrm{CH}^{+} \mathrm{BF}_{4}^{-}$(at $20^{\circ} \mathrm{C}$, cosolvent 0.2 vol- $\% \mathrm{CH}_{3} \mathrm{CN}$, stopped-flow, detection at $634 \mathrm{~nm}$ )

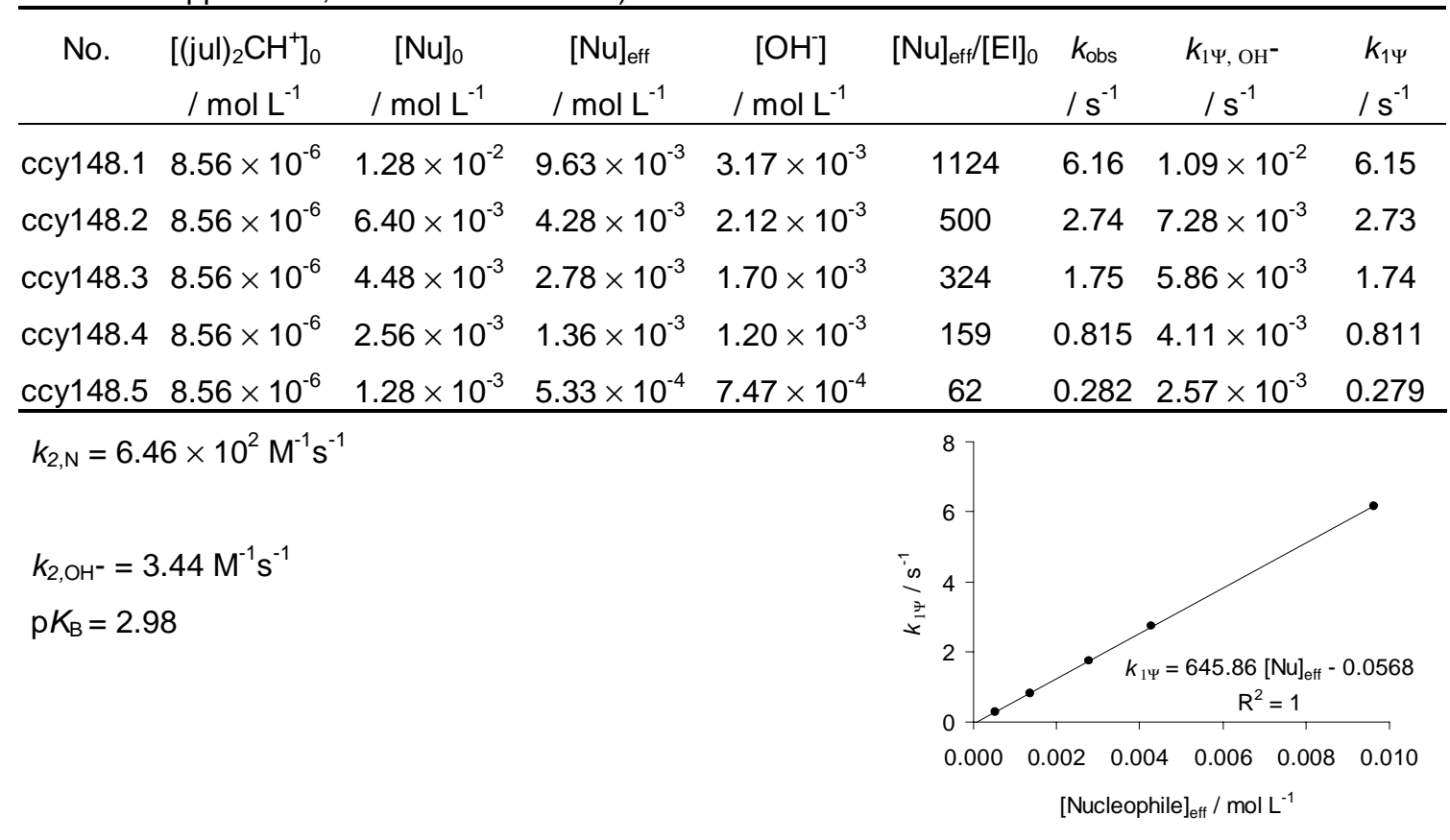


22.1.7. Reaction of Diethylamine (1t) with (lil) $)_{2} \mathrm{CH}^{+} \mathrm{BF}_{4}^{-}$(at $20^{\circ} \mathrm{C}$, cosolvent 0.2 vol- $\% \mathrm{CH}_{3} \mathrm{CN}$, stopped-flow, detection at $634 \mathrm{~nm}$ )

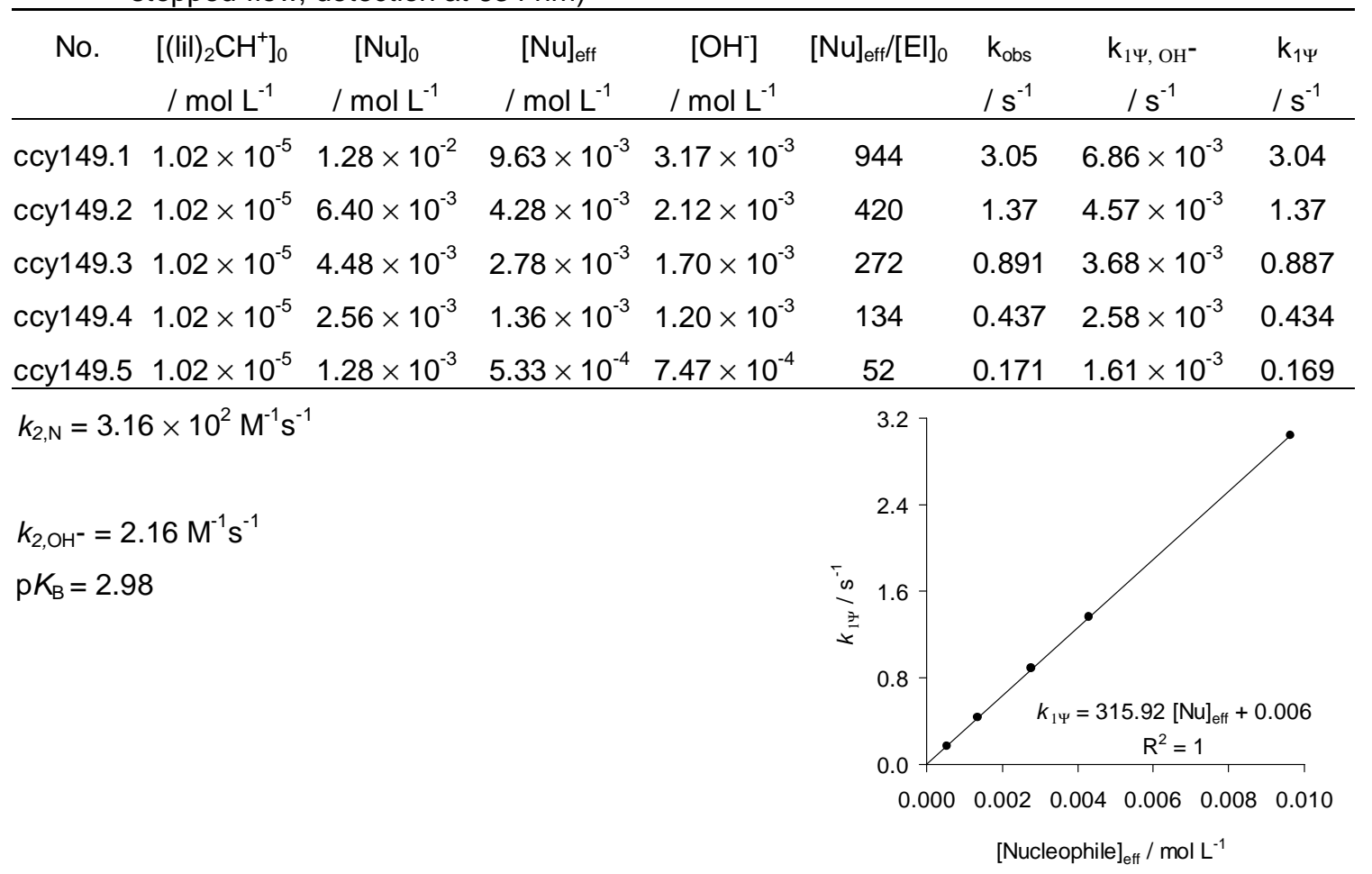

22.2. Reactivity parameters in water: $N=14.68 ; s=0.53$

\begin{tabular}{ccc}
\hline Reference electrophile & $E$ parameter & $k_{2, \mathrm{~N}}\left(20^{\circ} \mathrm{C}\right) / \mathrm{M}^{-1} \mathrm{~s}^{-1}$ \\
\hline (mor $)_{2} \mathrm{CH}^{+}$ & -5.53 & $7.92 \times 10^{4}$ \\
$(\mathrm{dma})_{2} \mathrm{CH}^{+}$ & -7.02 & $1.12 \times 10^{4}$ \\
$(\text { pyr })_{2} \mathrm{CH}^{+}$ & -7.69 & $4.88 \times 10^{3}$ \\
$(\text { thq })_{2} \mathrm{CH}^{+}$ & -8.22 & $2.84 \times 10^{3}$ \\
$\left(\right.$ ind ${ }_{2} \mathrm{CH}^{+}$ & -8.76 & $1.16 \times 10^{3}$ \\
$(\text { jul })_{2} \mathrm{CH}^{+}$ & -9.45 & $6.46 \times 10^{2}$ \\
$(\mathrm{lil})_{2} \mathrm{CH}^{+}$ & -10.04 & $3.16 \times 10^{2}$ \\
\hline
\end{tabular}

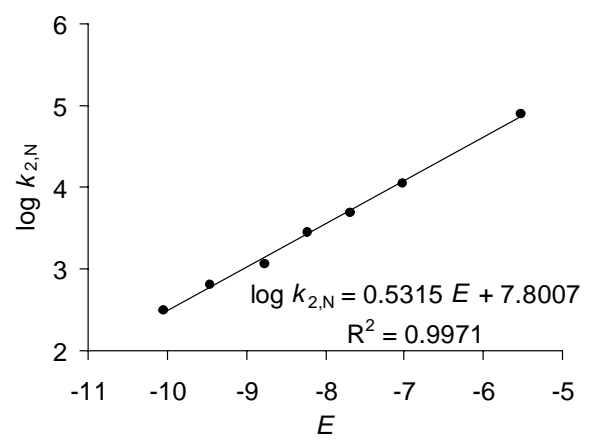




\section{Methylaminoacetonitrile (1u)}

23.1. Rate constants in water

23.1.1. Reaction of Methylaminoacetonitrile (1u) with $(\mathrm{dma})_{2} \mathrm{CH}^{+} \mathrm{BF}_{4}{ }_{4}^{-}$(at $20^{\circ} \mathrm{C}$, cosolvent 0.5 vol-\% $\mathrm{CH}_{3} \mathrm{CN}$, stopped-flow, detection at $610 \mathrm{~nm}$ )

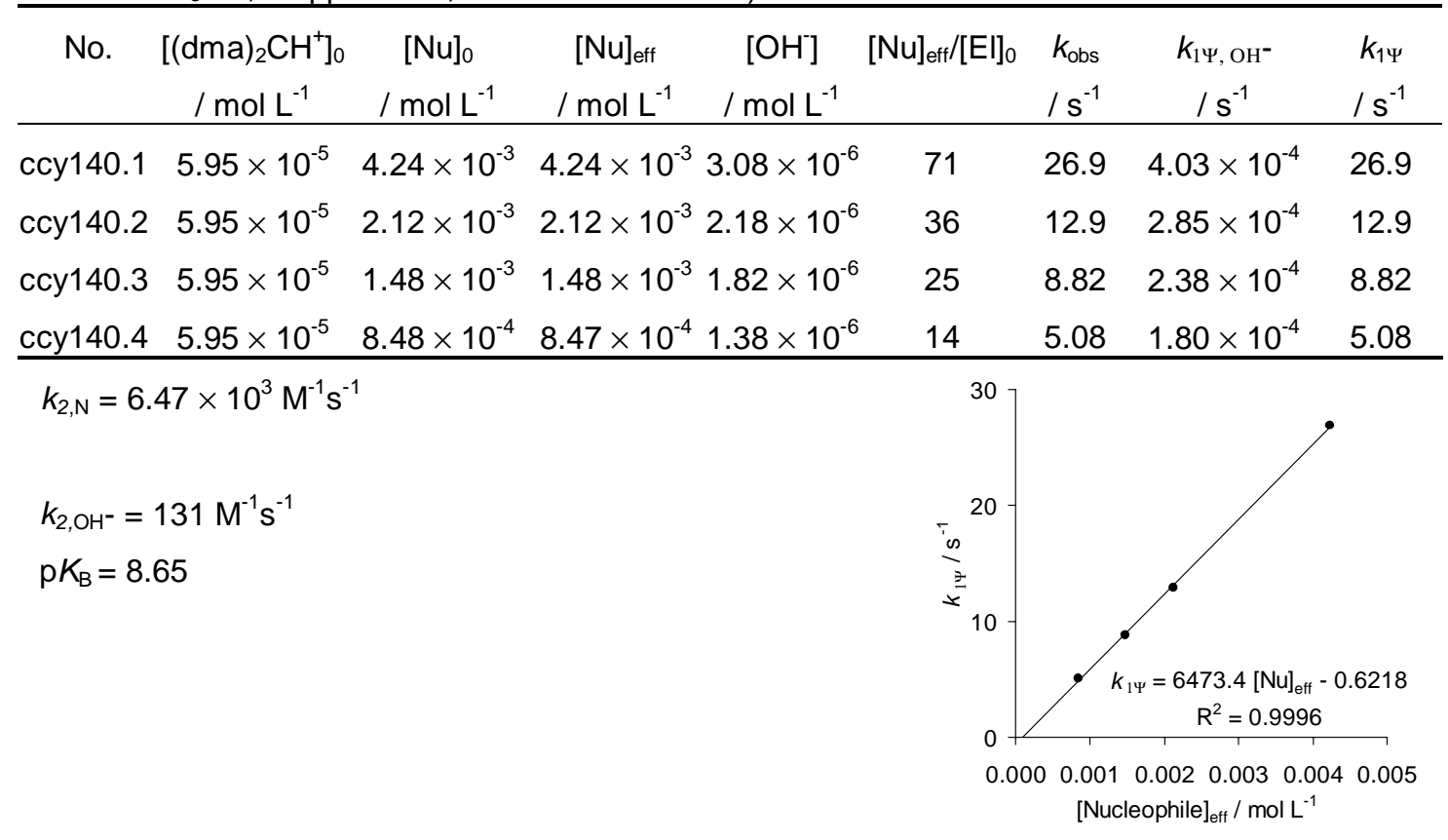

23.1.2. Reaction of Methylaminoacetonitrile (1u) with $(\mathrm{pyr})_{2} \mathrm{CH}^{+} \mathrm{BF}_{4}^{-}$(at $20^{\circ} \mathrm{C}$, cosolvent 0.1 vol-\% $\mathrm{CH}_{3} \mathrm{CN}$, stopped-flow, detection at $610 \mathrm{~nm}$ )

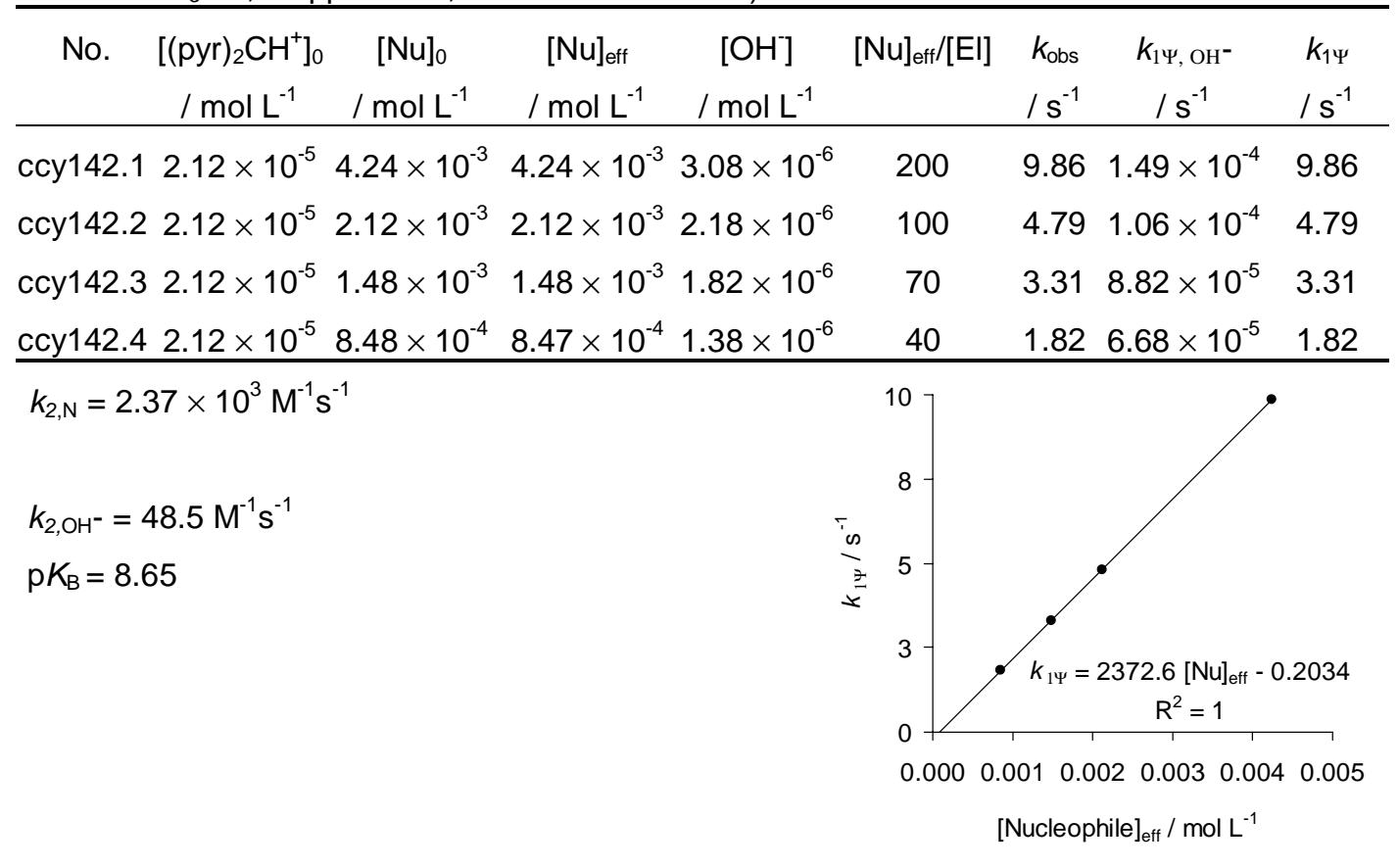


23.1.3. Reaction of Methylaminoacetonitrile (1u) with (thq) ${ }_{2} \mathrm{CH}^{+} \mathrm{BF}_{4}^{-}$(at $20^{\circ} \mathrm{C}$, cosolvent 0.4 vol-\% $\mathrm{CH}_{3} \mathrm{CN}$, stopped-flow, detection at $610 \mathrm{~nm}$ )

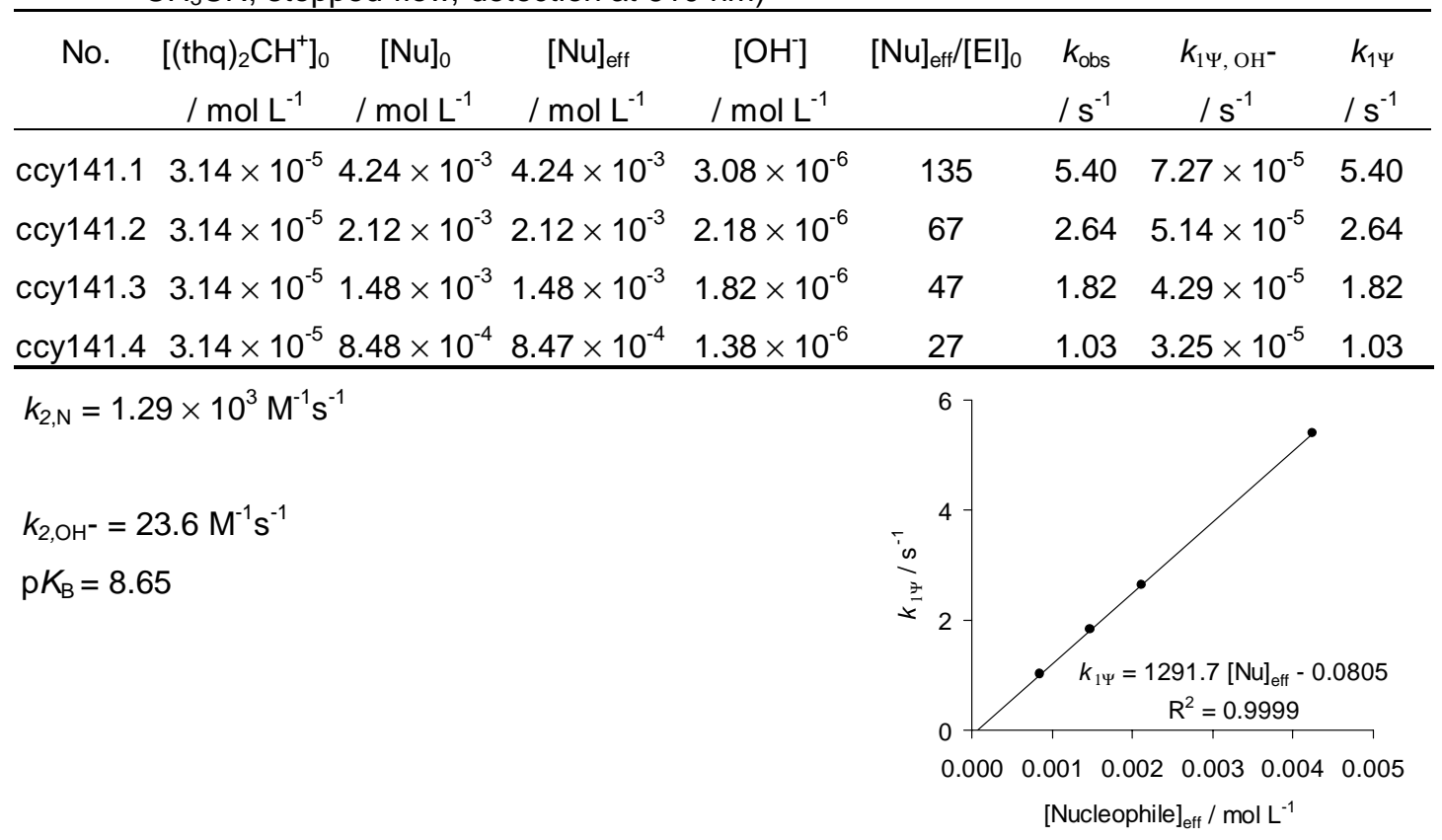

23.2. Reactivity parameters in water: $N=13.5 ; s=0.59$

\begin{tabular}{ccc}
\hline Reference electrophile & $E$ parameter & $k_{2, \mathrm{~N}}\left(20^{\circ} \mathrm{C}\right) / \mathrm{M}^{-1} \mathrm{~s}^{-1}$ \\
\hline$(\mathrm{dma})_{2} \mathrm{CH}^{+}$ & -7.02 & $6.47 \times 10^{3}$ \\
$(\mathrm{pyr})_{2} \mathrm{CH}^{+}$ & -7.69 & $2.37 \times 10^{3}$ \\
$(\text { thq })_{2} \mathrm{CH}^{+}$ & -8.22 & $1.29 \times 10^{3}$ \\
\hline
\end{tabular}

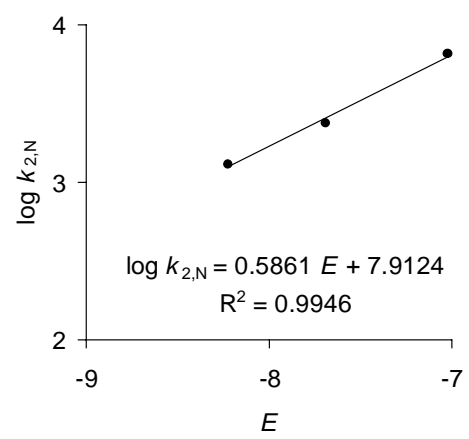




\section{2,2'-Iminodiethanol (1v)}

24.1. Rate constants in water

24.1.1. Reaction of 2,2'-Iminodiethanol (1v) with (dma) ${ }_{2} \mathrm{CH}^{+} \mathrm{BF}_{4}{ }^{-}$(at $20^{\circ} \mathrm{C}$, cosolvent 0.5 vol-\% $\mathrm{CH}_{3} \mathrm{CN}$, stopped-flow, detection at $610 \mathrm{~nm}$ )

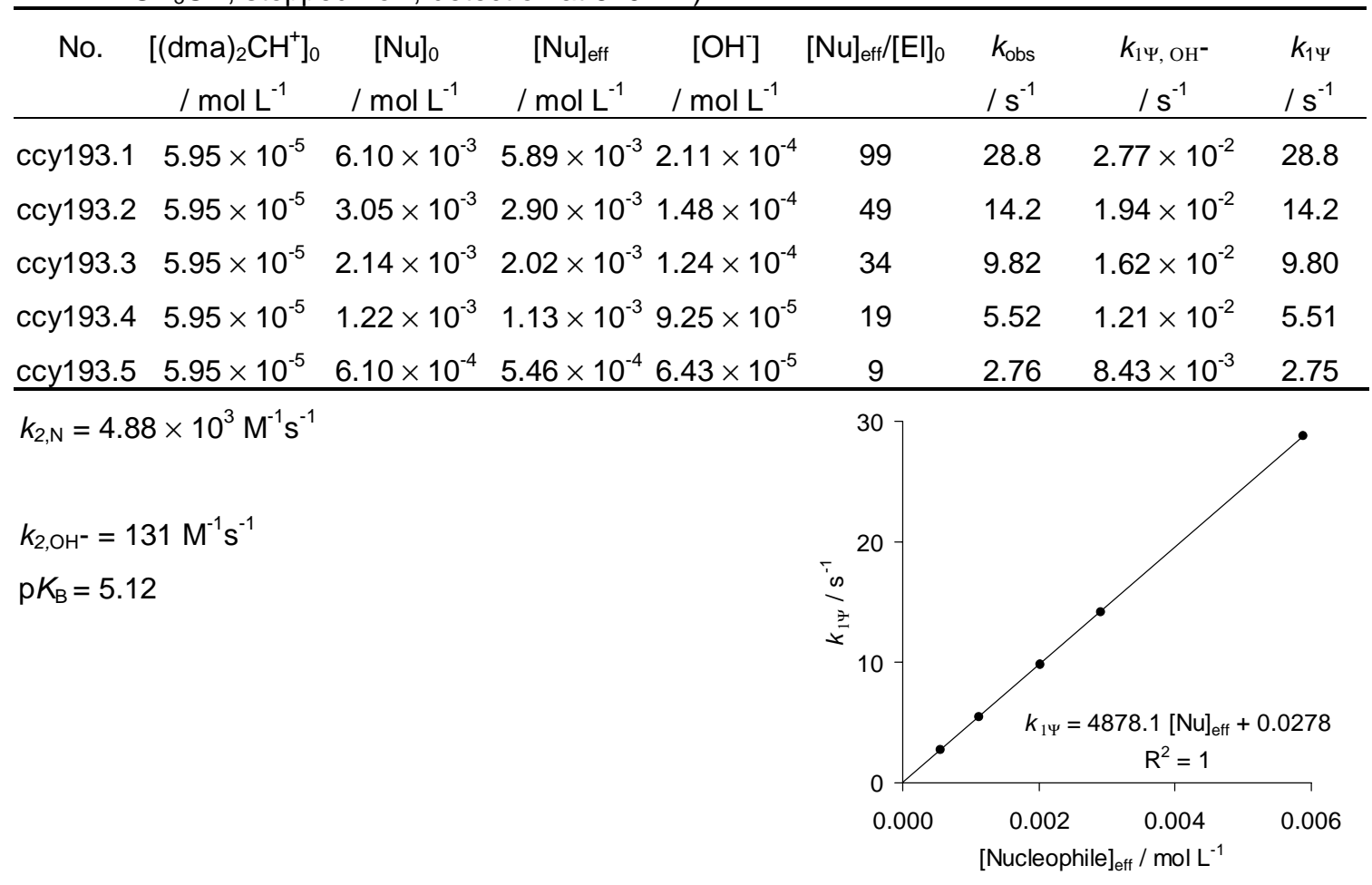

24.1.2. Reaction of 2,2'-Iminodiethanol (1v) with (pyr) $)_{2} \mathrm{CH}^{+} \mathrm{BF}_{4}^{-}$(at $20^{\circ} \mathrm{C}$, cosolvent 0.1 vol-\% $\mathrm{CH}_{3} \mathrm{CN}$, stopped-flow, detection at $610 \mathrm{~nm}$ )

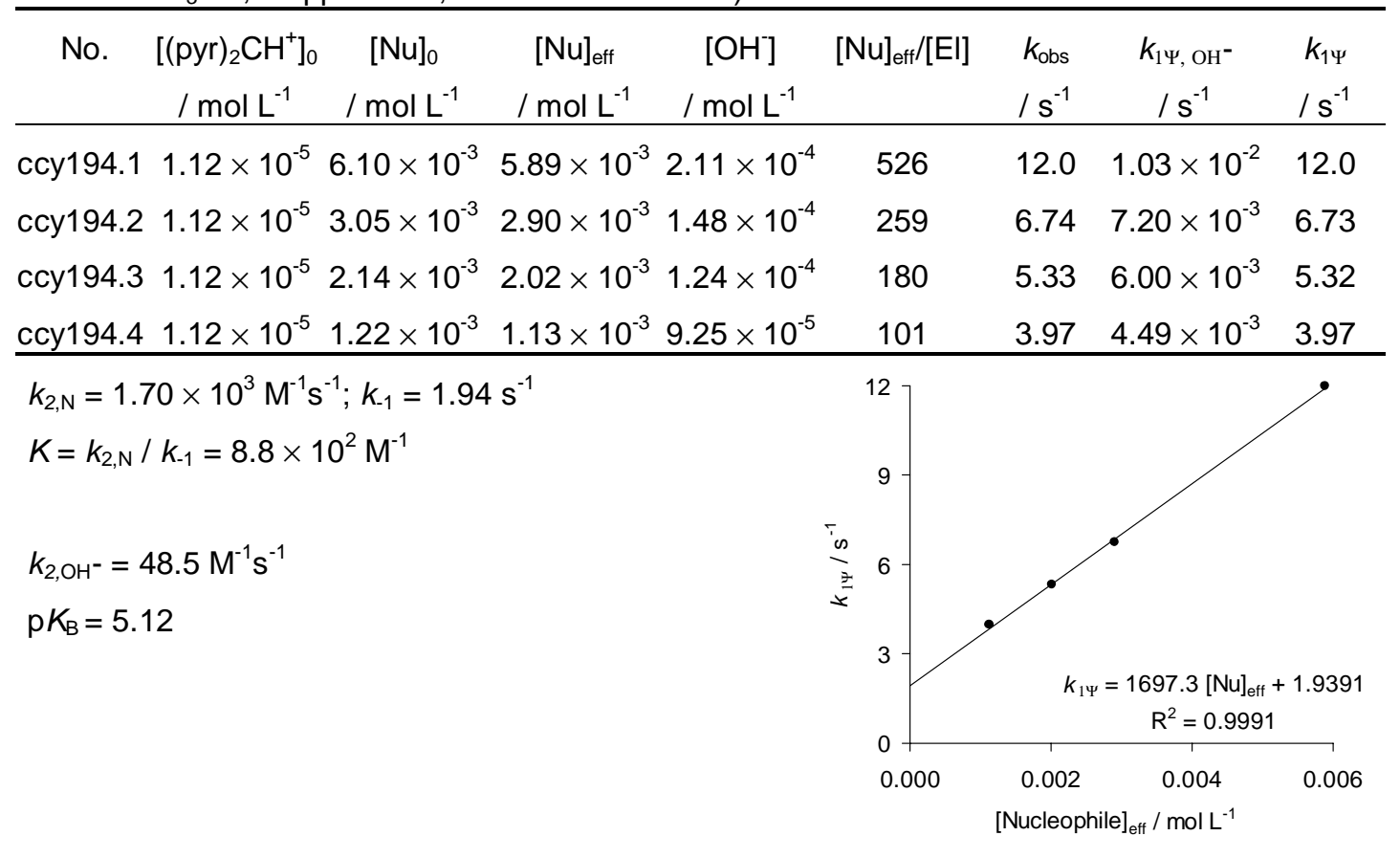


24.1.3. Reaction of $2,2^{\prime}-$ Iminodiethanol (1v) with (thq) ${ }_{2} \mathrm{CH}^{+} \mathrm{BF}_{4}{ }^{-}$(at $20^{\circ} \mathrm{C}$, cosolvent 0.2 vol-\% $\mathrm{CH}_{3} \mathrm{CN}$, stopped-flow, detection at $610 \mathrm{~nm}$ )

\begin{tabular}{|c|c|c|c|c|c|c|c|c|}
\hline No. & $\begin{array}{c}{\left[(\text { thq })_{2} \mathrm{CH}^{+}\right]_{0}} \\
/ \mathrm{mol} \mathrm{L}^{-1}\end{array}$ & $\begin{array}{c}{[\mathrm{Nu}]_{0}} \\
/ \mathrm{mol} \mathrm{L}^{-1}\end{array}$ & $\begin{array}{c}{[\mathrm{Nu}]_{\mathrm{eff}}} \\
/ \mathrm{mol} \mathrm{L}^{-1}\end{array}$ & $\begin{array}{c}{\left[\mathrm{OH}^{-}\right]} \\
/ \mathrm{mol} \mathrm{L}^{-1}\end{array}$ & {$[\mathrm{Nu}]_{\mathrm{eff}} /[\mathrm{El}]_{0}$} & $\begin{array}{l}k_{\mathrm{obs}} \\
/ \mathrm{s}^{-1}\end{array}$ & $\begin{array}{c}k_{1 \Psi, \mathrm{OH}^{-}} \\
/ \mathrm{s}^{-1} \\
\end{array}$ & $\begin{array}{l}k_{1 \Psi} \\
/ \mathrm{s}^{-1} \\
\end{array}$ \\
\hline ссу195.1 & $1.91 \times 10^{-5}$ & $6.10 \times 10^{-3}$ & $5.89 \times 10^{-3}$ & $2.11 \times 10^{-4}$ & 308 & 6.12 & $4.99 \times 10^{-3}$ & 6.12 \\
\hline сcy195.2 & $1.91 \times 10^{-5}$ & $3.05 \times 10^{-3}$ & $2.90 \times 10^{-3}$ & $1.48 \times 10^{-4}$ & 152 & 3.35 & $3.50 \times 10^{-3}$ & 3.35 \\
\hline ссу195.3 & $1.91 \times 10^{-5}$ & $2.14 \times 10^{-3}$ & $2.02 \times 10^{-3}$ & $1.24 \times 10^{-4}$ & 106 & 2.56 & $2.92 \times 10^{-3}$ & 2.56 \\
\hline сcy195.4 & $1.91 \times 10^{-5}$ & $1.22 \times 10^{-3}$ & $1.13 \times 10^{-3}$ & $9.25 \times 10^{-5}$ & 59 & 1.87 & $2.18 \times 10^{-3}$ & 1.87 \\
\hline \multirow{5}{*}{\multicolumn{9}{|c|}{$\begin{array}{l}k_{2, \mathrm{~N}}=9.01 \times 10^{2} \mathrm{M}^{-1} \mathrm{~s}^{-1} ; k_{-1}=0.785 \mathrm{~s}^{-1} \\
K=k_{2, \mathrm{~N}} / k_{-1}=1.1 \times 10^{3} \mathrm{M}^{-1} \\
k_{2, \mathrm{OH}}=23.6 \mathrm{M}^{-1} \mathrm{~s}^{-1} \\
\mathrm{p} K_{\mathrm{B}}=5.12\end{array}$}} \\
\hline & & & & & & & & \\
\hline & & & & & & & & \\
\hline & & & & & & & & \\
\hline & & & & & & & & \\
\hline & & & & & 0.000 & 0.002 & 0.004 & 0.006 \\
\hline & & & & & & & & \\
\hline
\end{tabular}

24.2. Reactivity parameters in water: $N=13.00 ; s=0.61$

\begin{tabular}{ccc}
\hline Reference electrophile & $E$ parameter & $k_{2, \mathrm{~N}}\left(20^{\circ} \mathrm{C}\right) / \mathrm{M}^{-1} \mathrm{~s}^{-1}$ \\
\hline$(\mathrm{dma})_{2} \mathrm{CH}^{+}$ & -7.02 & $4.88 \times 10^{3}$ \\
$(\mathrm{pyr})_{2} \mathrm{CH}^{+}$ & -7.69 & $1.70 \times 10^{3}$ \\
$(\text { thq })_{2} \mathrm{CH}^{+}$ & -8.22 & $9.01 \times 10^{2}$ \\
\hline
\end{tabular}

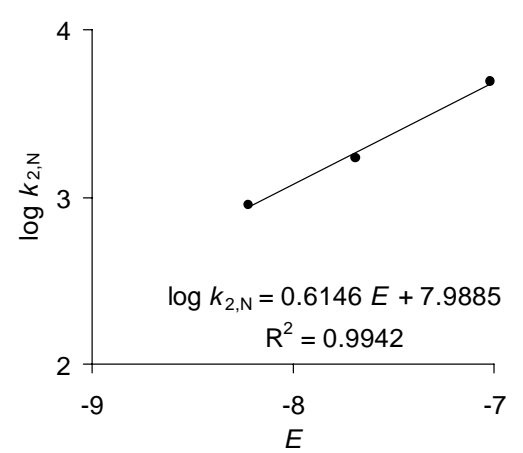




\section{Pyrrolidine (1w)}

25.1. Rate constants in water

25.1.1. Reaction of Pyrrolidine (1w) with $(\mathrm{dma})_{2} \mathrm{CH}^{+} \mathrm{BF}_{4}^{-}$(at $20{ }^{\circ} \mathrm{C}$, cosolvent: 0.4 vol- $\% \mathrm{CH}_{3} \mathrm{CN}$, stopped-flow, detection at $604 \mathrm{~nm}$ )

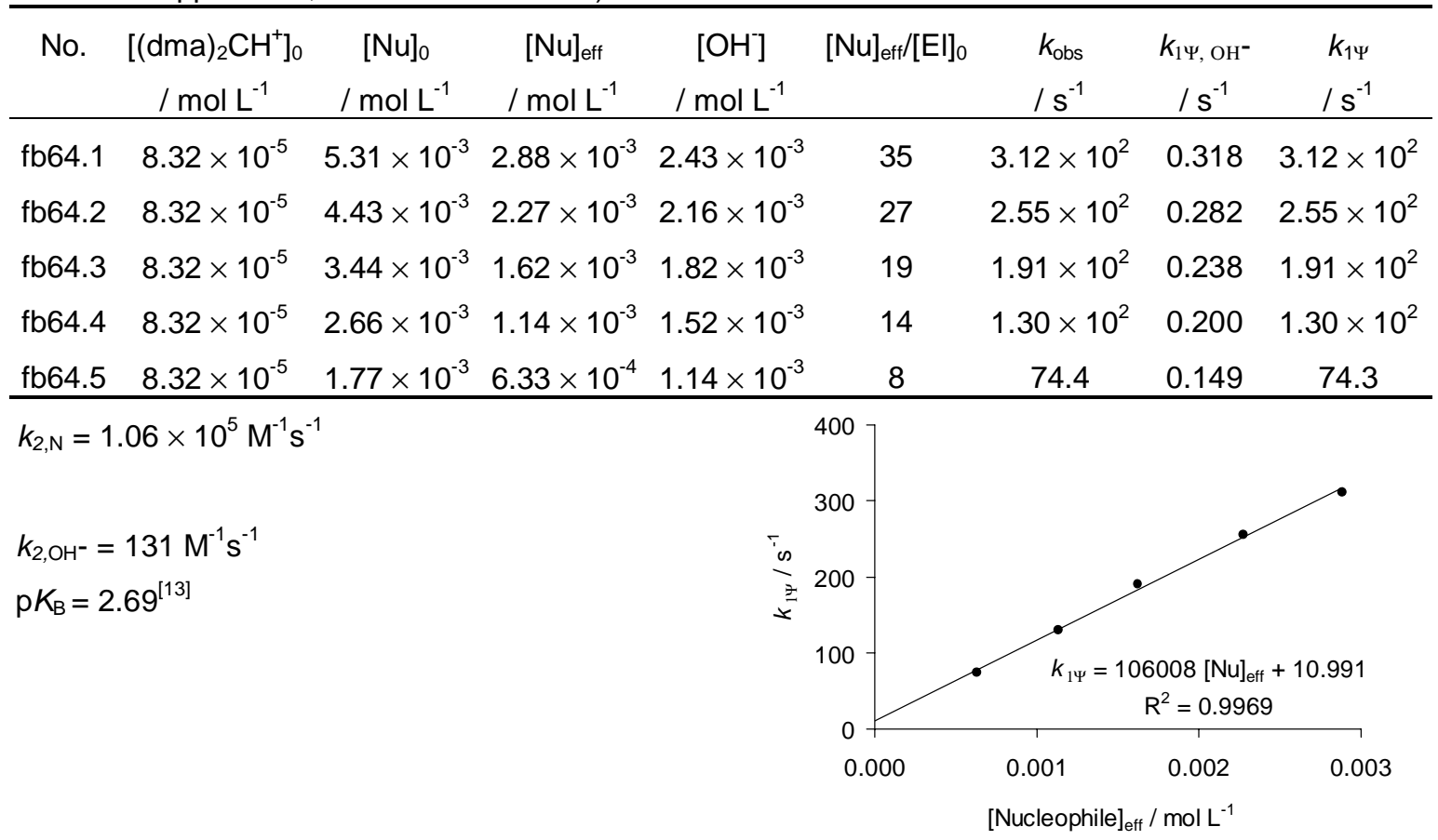

25.1.2. Reaction of Pyrrolidine (1w) with (pyr) ${ }_{2} \mathrm{CH}^{+} \mathrm{BF}_{4}^{-}$(at $20^{\circ} \mathrm{C}$, cosolvent: 0.2 vol- $\% \mathrm{CH}_{3} \mathrm{CN}$, stopped-flow, detection at $611 \mathrm{~nm}$ )

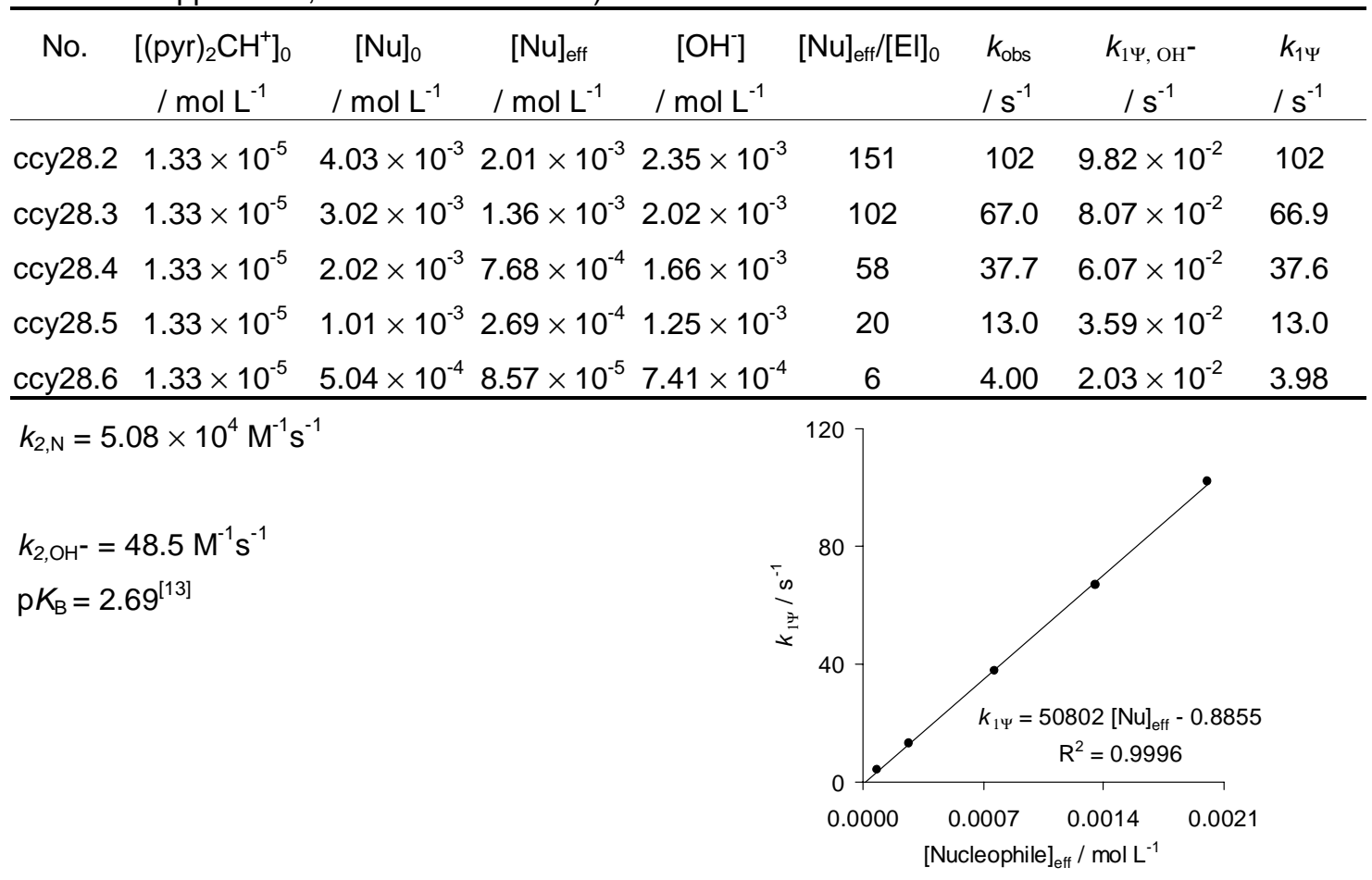


25.1.3. Reaction of Pyrrolidine (1w) with (thq) ${ }_{2} \mathrm{CH}^{+} \mathrm{BF}_{4}^{-}$(at $20{ }^{\circ} \mathrm{C}$, cosolvent: 0.4 vol- $\% \mathrm{CH}_{3} \mathrm{CN}$, stopped-flow, detection at $618 \mathrm{~nm}$ )

\begin{tabular}{|c|c|c|c|c|c|c|c|c|}
\hline No. & $\begin{array}{c}{\left[(\text { thq })_{2} \mathrm{CH}^{+}\right]_{0}} \\
/ \mathrm{mol} \mathrm{L}^{-1}\end{array}$ & $\begin{array}{c}{[\mathrm{Nu}]_{0}} \\
/ \mathrm{mol} \mathrm{L}^{-1}\end{array}$ & $\begin{array}{c}{[\mathrm{Nu}]_{\mathrm{eff}}} \\
/ \mathrm{mol} \mathrm{L}^{-1}\end{array}$ & $\begin{array}{c}{\left[\mathrm{OH}^{-}\right]} \\
/ \mathrm{mol} \mathrm{L}^{-1}\end{array}$ & {$[\mathrm{Nu}]_{\mathrm{eff}} /[\mathrm{El}]_{0}$} & $\begin{array}{l}k_{\mathrm{obs}} \\
/ \mathrm{s}^{-1}\end{array}$ & $\begin{array}{c}k_{1 \Psi, \mathrm{OH}^{-}} \\
/ \mathrm{s}^{-1}\end{array}$ & $\begin{array}{l}k_{1 \Psi} \\
/ s^{-1}\end{array}$ \\
\hline $\mathrm{fb} 60.1$ & $1.86 \times 10^{-5}$ & $8.85 \times 10^{-3}$ & $5.50 \times 10^{-3}$ & $3.35 \times 10^{-3}$ & 296 & $1.44 \times 10^{2}$ & $7.91 \times 10^{-2}$ & $1.44 \times 10^{2}$ \\
\hline $\mathrm{fb} 60.2$ & $1.86 \times 10^{-5}$ & $5.31 \times 10^{-3}$ & $2.88 \times 10^{-3}$ & $2.43 \times 10^{-3}$ & 155 & 76.4 & $5.73 \times 10^{-2}$ & 76.3 \\
\hline $\mathrm{fb} 60.3$ & $1.86 \times 10^{-5}$ & $4.43 \times 10^{-3}$ & $2.27 \times 10^{-3}$ & $2.16 \times 10^{-3}$ & 122 & 62.2 & $5.09 \times 10^{-2}$ & 62.1 \\
\hline fb59.1 & $1.86 \times 10^{-5}$ & $3.54 \times 10^{-3}$ & $1.69 \times 10^{-3}$ & $1.85 \times 10^{-3}$ & 91 & 45.6 & $4.38 \times 10^{-2}$ & 45.6 \\
\hline $\mathrm{fb} 60.4$ & $1.86 \times 10^{-5}$ & $3.44 \times 10^{-3}$ & $1.62 \times 10^{-3}$ & $1.82 \times 10^{-3}$ & 87 & 45.9 & $4.29 \times 10^{-2}$ & 45.9 \\
\hline $\mathrm{fb} 60.5$ & $1.86 \times 10^{-5}$ & $2.66 \times 10^{-3}$ & $1.14 \times 10^{-3}$ & $1.52 \times 10^{-3}$ & 61 & 31.1 & $3.60 \times 10^{-2}$ & 31.1 \\
\hline $\mathrm{fb} 60.6$ & $1.86 \times 10^{-5}$ & $1.77 \times 10^{-3}$ & $6.33 \times 10^{-4}$ & $1.14 \times 10^{-3}$ & 34 & 17.5 & $2.68 \times 10^{-2}$ & 17.5 \\
\hline $\mathrm{fb} 59.2$ & $1.86 \times 10^{-5}$ & $1.77 \times 10^{-3}$ & $6.33 \times 10^{-4}$ & $1.14 \times 10^{-3}$ & 34 & 17.0 & $2.68 \times 10^{-2}$ & 17.0 \\
\hline fb59.3 & $1.86 \times 10^{-5}$ & $1.06 \times 10^{-3}$ & $2.90 \times 10^{-4}$ & $7.70 \times 10^{-4}$ & 16 & 7.42 & $1.82 \times 10^{-2}$ & 7.40 \\
\hline $\mathrm{fb} 60.7$ & $1.86 \times 10^{-5}$ & $8.85 \times 10^{-4}$ & $2.18 \times 10^{-4}$ & $6.67 \times 10^{-4}$ & 12 & 6.00 & $1.57 \times 10^{-2}$ & 5.98 \\
\hline $\mathrm{fb} 60.8$ & $1.86 \times 10^{-5}$ & $5.31 \times 10^{-4}$ & $9.37 \times 10^{-5}$ & $4.37 \times 10^{-4}$ & 5 & 2.45 & $1.03 \times 10^{-2}$ & 2.44 \\
\hline
\end{tabular}

$$
\begin{aligned}
& k_{2, \mathrm{OH}^{-}}=23.6 \mathrm{M}^{-1} \mathrm{~s}^{-1} \\
& \mathrm{p} K_{\mathrm{B}}=2.69^{[13]}
\end{aligned}
$$

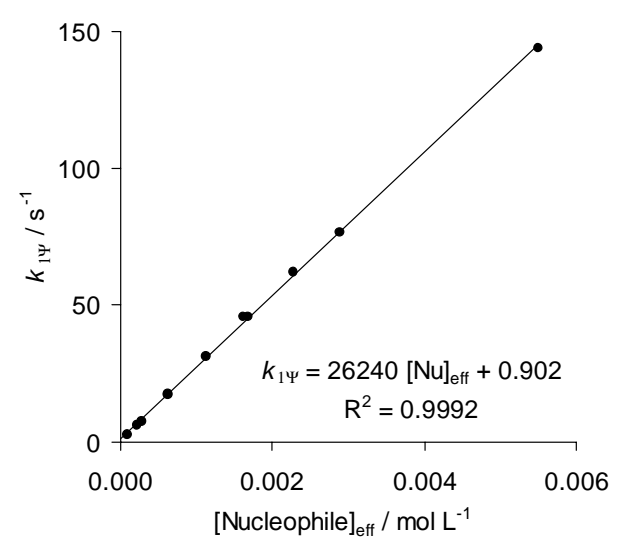


25.1.4. Reaction of Pyrrolidine (1w) with (ind) ${ }_{2} \mathrm{CH}^{+} \mathrm{BF}_{4}^{-}$(at $20^{\circ} \mathrm{C}$, cosolvent: 0.2 vol- $\% \mathrm{CH}_{3} \mathrm{CN}$, stopped-flow, detection at $614 \mathrm{~nm}$ )

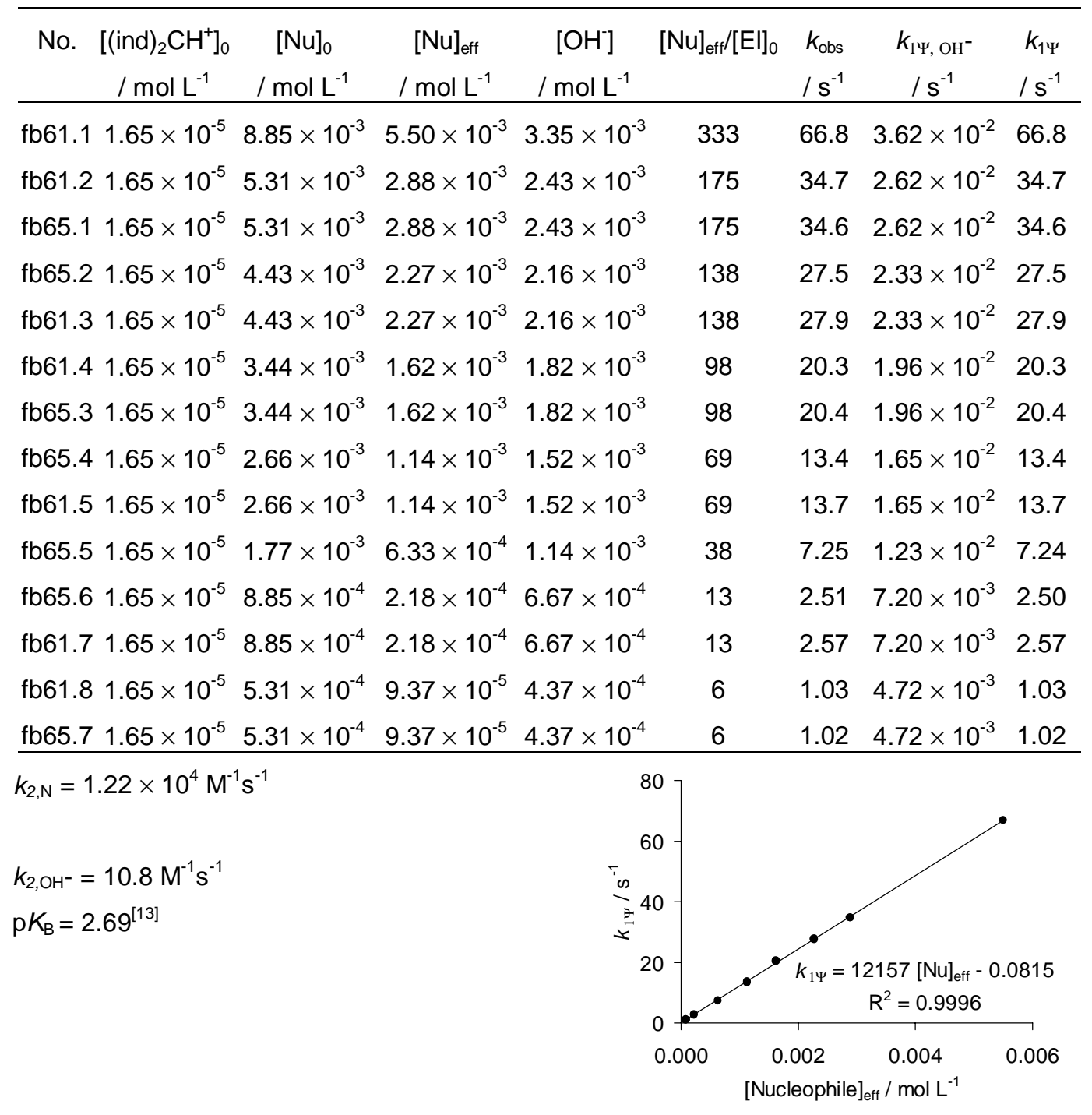


25.1.5. Reaction of Pyrrolidine (1w) with $(\mathrm{jul})_{2} \mathrm{CH}^{+} \mathrm{BF}_{4}{ }^{-}$(at $20{ }^{\circ} \mathrm{C}$, cosolvent: 0.2 vol- $\% \mathrm{CH}_{3} \mathrm{CN}$, stopped-flow, detection at $634 \mathrm{~nm}$ )

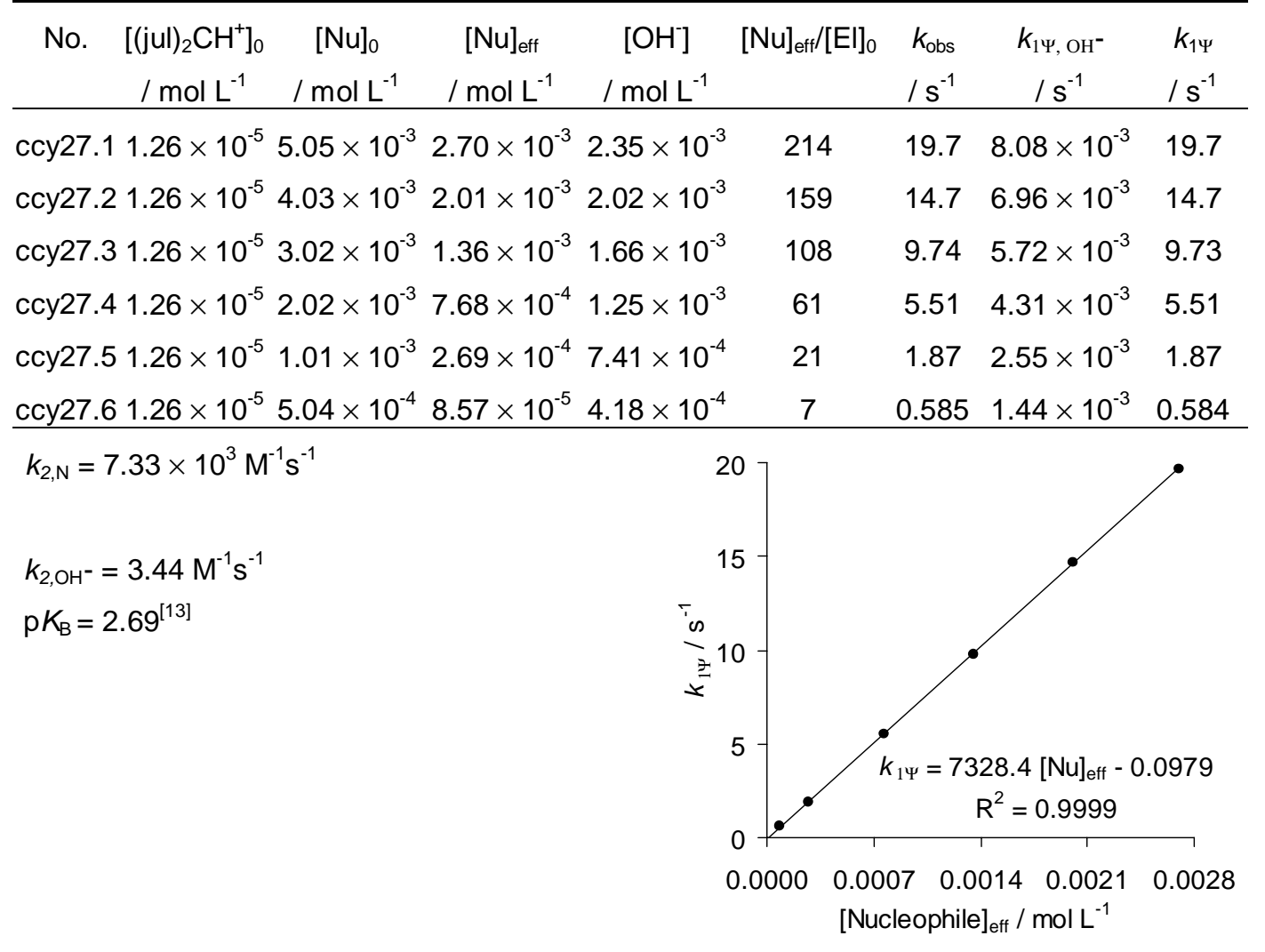


25.1.6. Reaction of Pyrrolidine (1w) with (lil) $)_{2} \mathrm{CH}^{+} \mathrm{BF}_{4}{ }_{4}$ (at $20^{\circ} \mathrm{C}$, cosolvent: 0.4 vol- $\% \mathrm{CH}_{3} \mathrm{CN}$, stopped-flow, detection at $634 \mathrm{~nm}$ )

\begin{tabular}{|c|c|c|c|c|c|c|c|c|}
\hline No. & $\begin{array}{c}{\left[(\mathrm{lil})_{2} \mathrm{CH}^{+}\right]_{0}} \\
/ \mathrm{mol} \mathrm{L}^{-1}\end{array}$ & $\begin{array}{c}{[\mathrm{Nu}]_{0}} \\
/ \mathrm{mol} \mathrm{L}^{-1}\end{array}$ & $\begin{array}{c}{[\mathrm{Nu}]_{\mathrm{eff}}} \\
/ \mathrm{mol} \mathrm{L}^{-1}\end{array}$ & $\begin{array}{c}{\left[\mathrm{OH}^{-}\right]} \\
/ \mathrm{mol} \mathrm{L}^{-1}\end{array}$ & ]o & $\begin{array}{l}k_{\mathrm{obs}} \\
/ \mathrm{s}^{-1}\end{array}$ & $\begin{array}{c}k_{1 \Psi, \mathrm{OH}^{-}} \\
/ \mathrm{s}^{-1} \\
\end{array}$ & $\begin{array}{l}k_{1 \Psi} \\
/ \mathrm{s}^{-1}\end{array}$ \\
\hline & 1 & 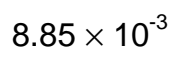 & $5.50 \times 10^{-3}$ & $3.35 \times 10^{-}$ & 304 & 18.8 & $7.24 \times 10^{-3}$ & 18.8 \\
\hline 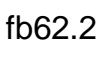 & & $5.31 \times 1$ & $2.88 \times 10^{-3}$ & $2.43 \times 10^{-3}$ & 59 & 9.52 & $5.24 \times 10^{-3}$ & 9.51 \\
\hline b3. & $1.81 \times 10^{-5}$ & $5.31 \times 10^{-3}$ & $2.88 \times 10^{-3}$ & $2.43 \times 10^{-3}$ & 59 & 9.80 & $5.24 \times 10^{-3}$ & 9.79 \\
\hline 63 & $1.81 \times 10^{-5}$ & $4.43 \times 10^{-3}$ & $2.27 \times 10^{-3}$ & $2.16 \times 10^{-3}$ & 26 & 7.71 & $4.66 \times 10^{-3}$ & 7.7 \\
\hline L & $31.81>$ & 4.43 & 2.27 & $2.16 \times 10^{-3}$ & 16 & 7.68 & $4.66 \times 10^{-3}$ & 7.68 \\
\hline 62 & $+1.81 \times 1$ & $3.44 \times$ & $1.62 \times 10^{-3}$ & $1.82 \times 10^{-3}$ & 90 & 5.51 & $3.93 \times 10^{-3}$ & 5.51 \\
\hline 63 & $31.81 \times 10^{-5}$ & $3.44 \times 1$ & $1.62 \times 10^{-3}$ & $1.82 \times 10^{-3}$ & 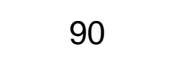 & 5.68 & $3.93 \times 10^{-3}$ & 5.68 \\
\hline O & $1.81 \times 10^{-5}$ & $2.66 \times$ & $1.14 \times 10^{-3}$ & $1.52 \times 10^{-3}$ & 63 & 3.78 & $3.29 \times 10^{-3}$ & 3.78 \\
\hline fb62 & $1.81 x$ & 2.66 & 1.14 & $1.52 \times 10^{-3}$ & 6 & 3.76 & 3.2 & 3.76 \\
\hline fb62. & $1.81 \times 10^{-5}$ & $1.77 \times$ & $6.33 \times 1$ & $1.14 \times 10^{-3}$ & 35 & 2.05 & $2.46 \times 10^{-3}$ & 2.05 \\
\hline fb63. & $1.81 \times 10^{-5}$ & $1.77 \times 1$ & $6.33 \times 10^{-4}$ & $1.14 \times 10^{-3}$ & 3 & 2.07 & $2.46 \times 10^{-3}$ & 2.07 \\
\hline $\mathrm{fb} 63$ & $1.81 \times 10^{-5}$ & & $2.18 \times 10^{-4}$ & $6.67 \times 10^{-4}$ & 12 & 0.721 & $1.44 \times 10^{-3}$ & 0.72 \\
\hline $\mathrm{fb} 62$ & $1.81 \times 10^{-5}$ & $8.85 \times 10^{-4}$ & $2.18 \times 10^{-4}$ & $6.67 \times 10^{-4}$ & 12 & 0.701 & $1.44 \times 10^{-3}$ & 0.700 \\
\hline $\mathrm{fb} 62$ & $31.81 \times 10^{-5}$ & $5.31 \times 10^{-4}$ & $9.37 \times 10^{-5}$ & $4.37 \times 10^{-4}$ & 5 & 0.269 & $9.45 \times 10^{-4}$ & 0.268 \\
\hline 063.7 & $1.81 \times 10^{-5}$ & $5.31 \times 10^{-4}$ & $9.37 \times 10^{-5}$ & $4.37 \times 10^{-4}$ & 5 & 0.306 & $9.45 \times 10^{-4}$ & 0.30 \\
\hline
\end{tabular}

$k_{2, \mathrm{~N}}=3.41 \times 10^{3} \mathrm{M}^{-1} \mathrm{~s}^{-1}$

$k_{2, \mathrm{OH}^{-}}=2.16 \mathrm{M}^{-1} \mathrm{~s}^{-1}$

$\mathrm{p} K_{\mathrm{B}}=2.69^{[13]}$

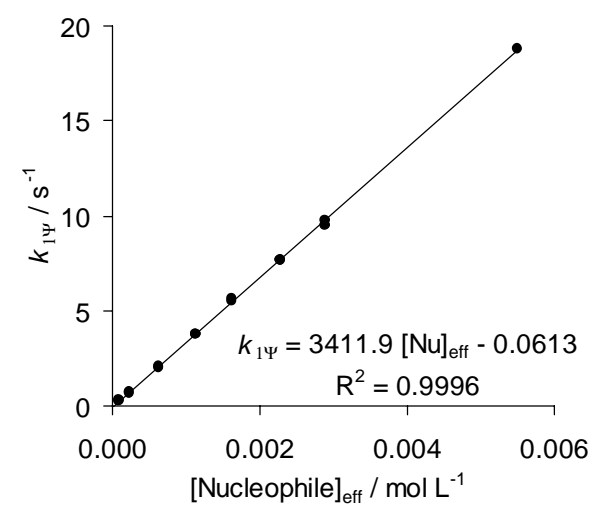

25.2. Reactivity parameters in water: $N=17.21 ; s=0.49$

\begin{tabular}{ccc}
\hline Reference electrophile & $E$ parameter & $k_{2, \mathrm{~N}}\left(20^{\circ} \mathrm{C}\right) / \mathrm{M}^{-1} \mathrm{~s}^{-1}$ \\
\hline$(\mathrm{dma})_{2} \mathrm{CH}^{+}$ & -7.02 & $1.06 \times 10^{5}$ \\
$(\mathrm{pyr})_{2} \mathrm{CH}^{+}$ & -7.69 & $5.08 \times 10^{4}$ \\
$(\text { thq })_{2} \mathrm{CH}^{+}$ & -8.22 & $2.62 \times 10^{4}$ \\
$(\text { ind })_{2} \mathrm{CH}^{+}$ & -8.76 & $1.22 \times 10^{4}$ \\
$(\text { jul })_{2} \mathrm{CH}^{+}$ & -9.45 & $7.33 \times 10^{3}$ \\
$(\text { (lil })_{2} \mathrm{CH}^{+}$ & -10.04 & $3.41 \times 10^{3}$ \\
\hline
\end{tabular}

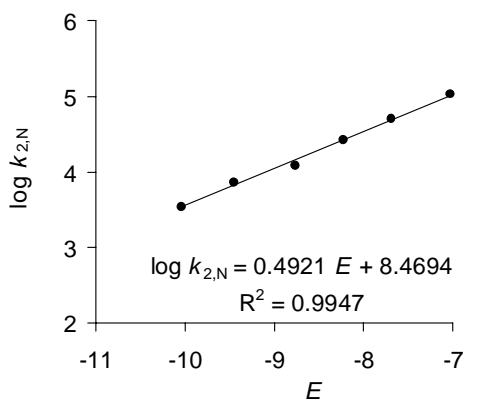




\section{Piperidine (1x)}

26.1. Rate constants in water

26.1.1. Reaction of Piperidine (1x) with (dma) ${ }_{2} \mathrm{CH}^{+} \mathrm{BF}_{4}{ }_{4}$ (at $20{ }^{\circ} \mathrm{C}$, cosolvent: 0.4 vol-\% $\mathrm{CH}_{3} \mathrm{CN}$, stopped-flow, detection at $604 \mathrm{~nm}$ )

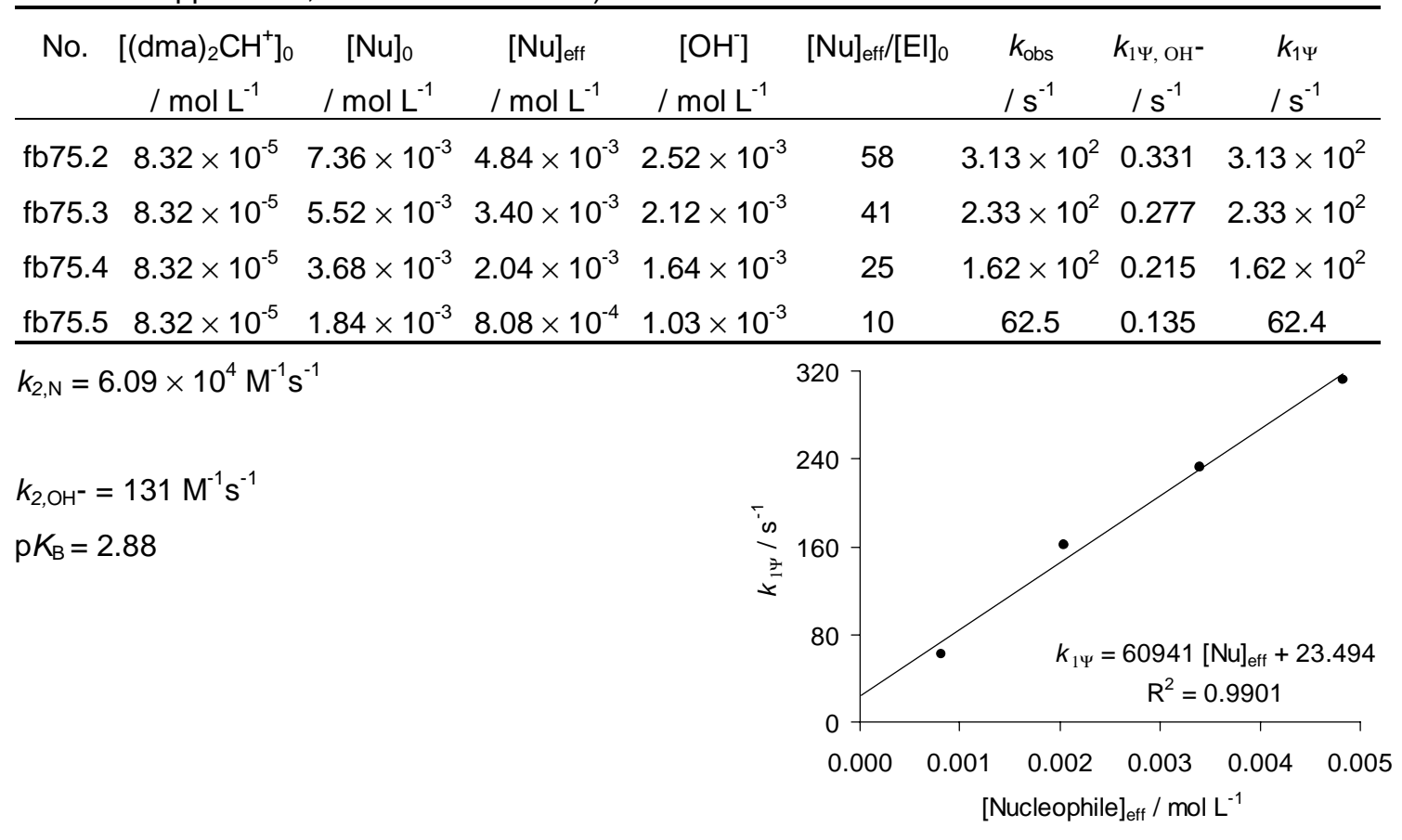

26.1.2. Reaction of Piperidine (1x) with (pyr) ${ }_{2} \mathrm{CH}^{+} \mathrm{BF}_{4}{ }^{-}$(at $20^{\circ} \mathrm{C}$, cosolvent: 0.2 vol- $\% \mathrm{CH}_{3} \mathrm{CN}$, stopped-flow, detection at $611 \mathrm{~nm}$ )

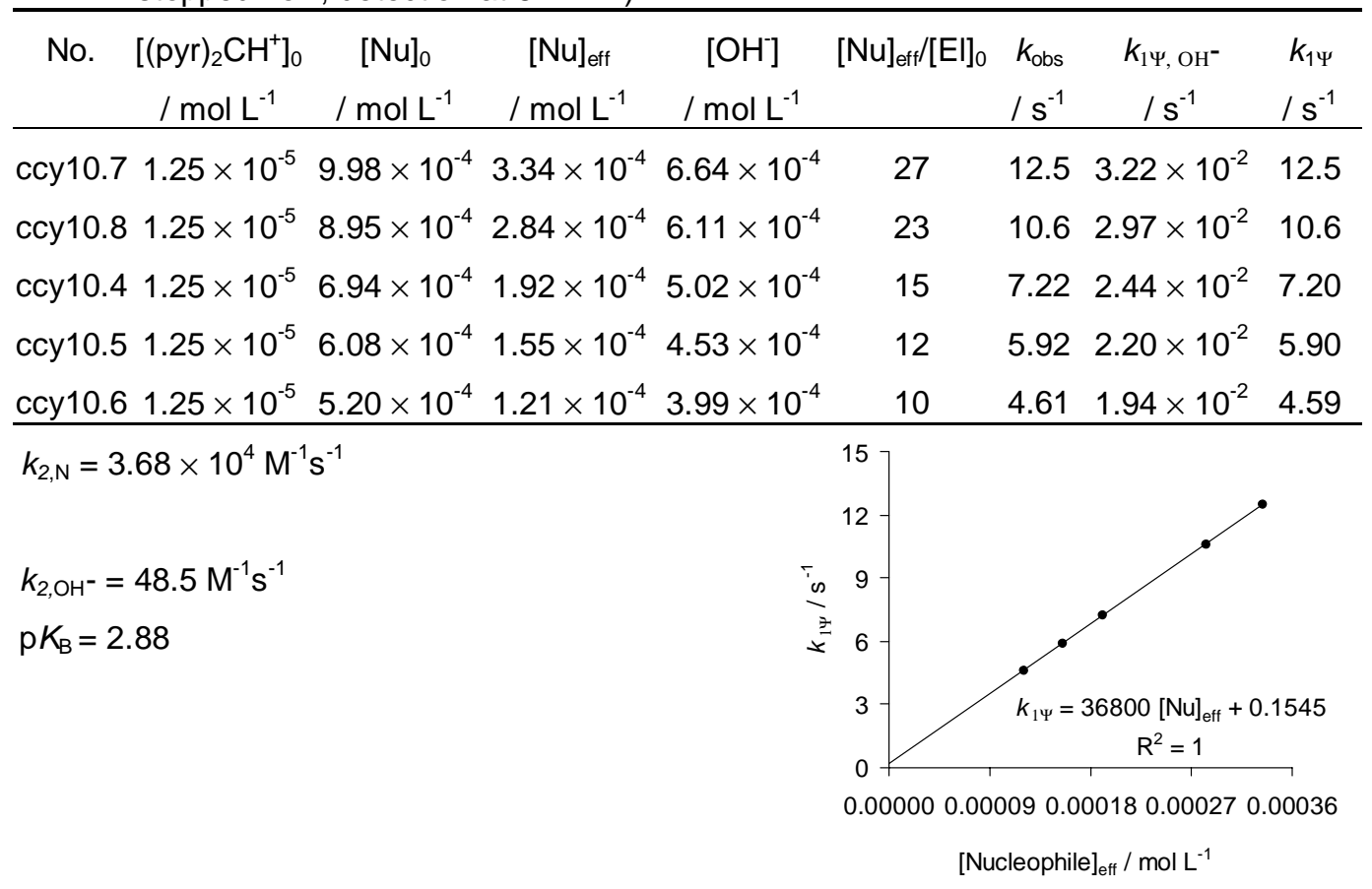


26.1.3. Reaction of Piperidine (1x) with (thq) ${ }_{2} \mathrm{CH}^{+} \mathrm{BF}_{4}^{-}$(at $20^{\circ} \mathrm{C}$, cosolvent: 0.2 vol- $\% \mathrm{CH}_{3} \mathrm{CN}$, stopped-flow, detection at $618 \mathrm{~nm}$ )

\begin{tabular}{ccccccccc}
\hline No. & $\begin{array}{c}{\left[(\text { thq })_{2} \mathrm{CH}^{+}\right]_{0}} \\
/ \mathrm{mol} \mathrm{L}^{-1}\end{array}$ & $\begin{array}{c}{[\mathrm{Nu}]_{0}} \\
/ \mathrm{mol} \mathrm{L}^{-1}\end{array}$ & $\begin{array}{c}{[\mathrm{Nu}]_{\mathrm{eff}}} \\
/ \mathrm{mol} \mathrm{L}^{-1}\end{array}$ & $\begin{array}{c}{[\mathrm{OH}]} \\
/ \mathrm{mol} \mathrm{L}^{-1}\end{array}$ & $\begin{array}{c}{[\mathrm{Nu}]_{\mathrm{eff}} /[\mathrm{E}]_{0}} \\
k_{\mathrm{obs}}\end{array}$ & $\begin{array}{c}k_{1 \Psi, \mathrm{OH}^{-}} \\
/ \mathrm{s}^{-1}\end{array}$ & $\begin{array}{c}k_{1 \Psi} \\
/ \mathrm{s}^{-1}\end{array}$ & $/ \mathrm{s}^{-1}$ \\
\hline ccy8.1 & $1.58 \times 10^{-5}$ & $2.60 \times 10^{-3}$ & $1.29 \times 10^{-3}$ & $1.31 \times 10^{-3}$ & 82 & 33.9 & $3.08 \times 10^{-2}$ & 33.9 \\
ccy8.2 & $1.58 \times 10^{-5}$ & $1.73 \times 10^{-3}$ & $7.41 \times 10^{-4}$ & $9.89 \times 10^{-4}$ & 47 & 19.4 & $2.33 \times 10^{-2}$ & 19.4 \\
ccy8.3 & $1.58 \times 10^{-5}$ & $8.65 \times 10^{-4}$ & $2.69 \times 10^{-4}$ & $5.96 \times 10^{-4}$ & 17 & 6.85 & $1.41 \times 10^{-2}$ & 6.84 \\
ccy8.4 & $1.58 \times 10^{-5}$ & $6.95 \times 10^{-4}$ & $1.92 \times 10^{-4}$ & $5.03 \times 10^{-4}$ & 12 & 4.83 & $1.19 \times 10^{-2}$ & 4.82 \\
ccy8.5 & $1.58 \times 10^{-5}$ & $6.05 \times 10^{-4}$ & $1.54 \times 10^{-4}$ & $4.51 \times 10^{-4}$ & 10 & 3.89 & $1.06 \times 10^{-2}$ & 3.88 \\
ccy8.6 & $1.58 \times 10^{-5}$ & $5.20 \times 10^{-4}$ & $1.21 \times 10^{-4}$ & $3.99 \times 10^{-4}$ & 8 & 2.92 & $9.42 \times 10^{-3}$ & 2.91 \\
\hline
\end{tabular}

$k_{2, \mathrm{~N}}=2.64 \times 10^{4} \mathrm{M}^{-1} \mathrm{~s}^{-1}$

$k_{2, \mathrm{OH}}=23.6 \mathrm{M}^{-1} \mathrm{~s}^{-1}$

$\mathrm{p} K_{\mathrm{B}}=2.88$

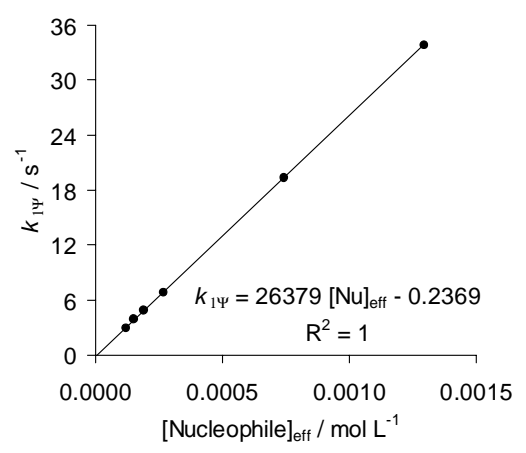

26.1.4. Reaction of Piperidine (1x) with (ind) ${ }_{2} \mathrm{CH}^{+} \mathrm{BF}_{4}^{-}$(at $20{ }^{\circ} \mathrm{C}$, cosolvent: 0.4 vol- $\% \mathrm{CH}_{3} \mathrm{CN}$, stopped-flow, detection at $614 \mathrm{~nm}$ )

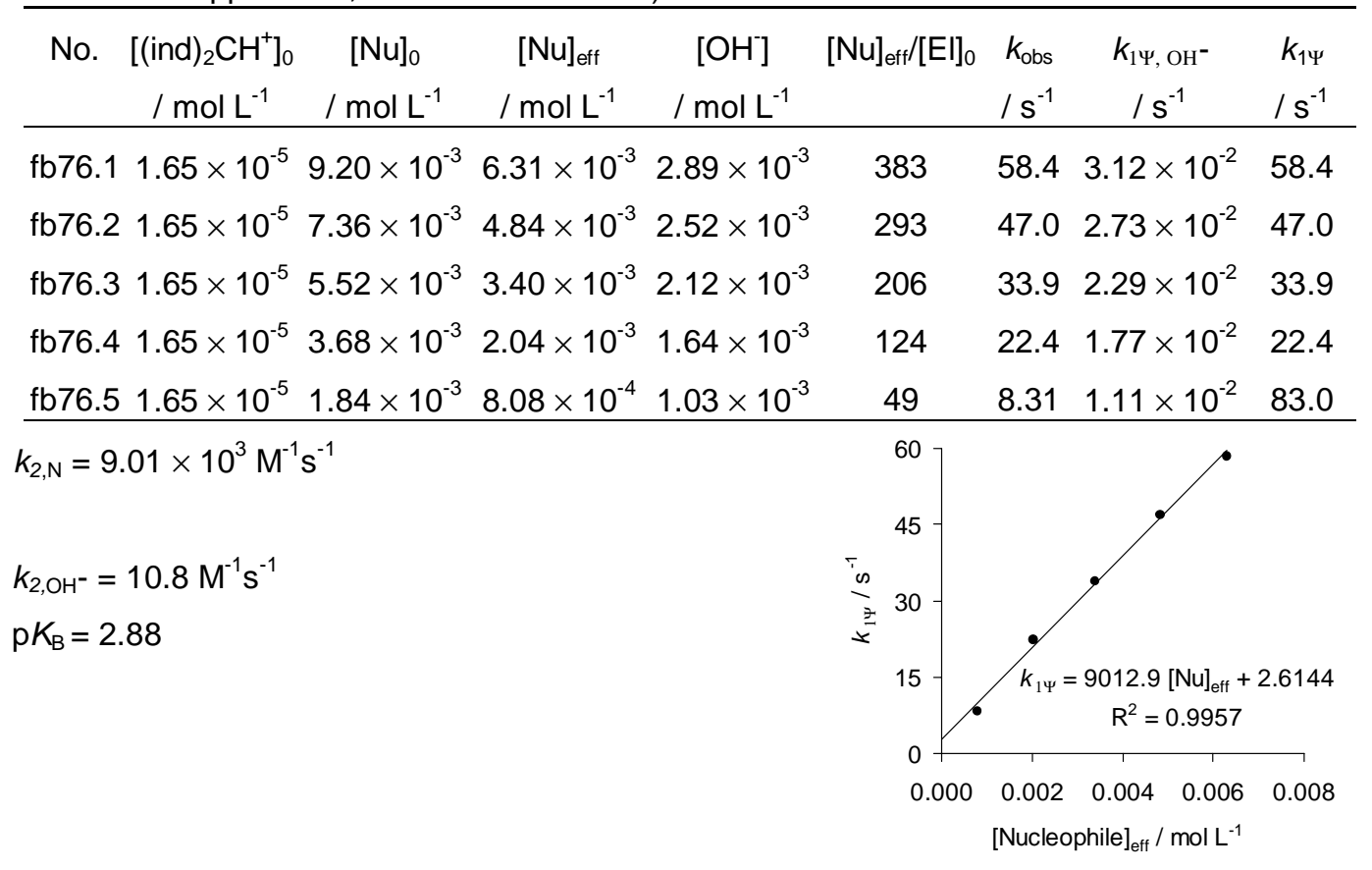


26.1.5. Reaction of Piperidine (1x) with (jul) ${ }_{2} \mathrm{CH}^{+} \mathrm{BF}_{4}{ }_{4}$ (at $20^{\circ} \mathrm{C}$, cosolvent: 0.2 vol- $\% \mathrm{CH}_{3} \mathrm{CN}$, stopped-flow, detection at $634 \mathrm{~nm}$ )

\begin{tabular}{|c|c|c|c|c|c|c|c|}
\hline $\begin{array}{c}\text { No. } \quad\left[(\mathrm{jul})_{2} \mathrm{CH}^{+}\right]_{0} \\
/ \mathrm{mol} \mathrm{L}^{-1}\end{array}$ & $\begin{array}{c}{[\mathrm{Nu}]_{0}} \\
/ \mathrm{mol} \mathrm{L}^{-1}\end{array}$ & $\begin{array}{c}{[\mathrm{Nu}]_{\mathrm{eff}}} \\
/ \mathrm{mol} \mathrm{L}^{-1}\end{array}$ & $\begin{array}{c}{\left[\mathrm{OH}^{-}\right]} \\
/ \mathrm{mol} \mathrm{L}^{-1}\end{array}$ & {$[\mathrm{Nu}]_{\mathrm{eff}} /[\mathrm{El}]_{0}$} & $\begin{array}{l}k_{\mathrm{obs}} \\
/ \mathrm{s}^{-1}\end{array}$ & $\begin{array}{c}k_{1 \Psi, \mathrm{OH}^{-}} \\
/ \mathrm{s}^{-1}\end{array}$ & $\begin{array}{l}k_{1 \Psi} \\
/ s^{-1}\end{array}$ \\
\hline ccy7.1 $1.26 \times 10^{-5}$ & $2.60 \times 10^{-3}$ & $1.29 \times 10^{-3}$ & $1.31 \times 10^{-3}$ & 103 & 9.00 & $4.49 \times 10^{-3}$ & 9.00 \\
\hline ccy7.2 $1.26 \times 10^{-5}$ & $1.73 \times 10^{-3}$ & $7.41 \times 10^{-4}$ & $9.89 \times 10^{-4}$ & 59 & 5.23 & $3.40 \times 10^{-3}$ & 5.23 \\
\hline $\operatorname{ccy} 7.31 .26 \times 10^{-5}$ & $8.65 \times 10^{-4}$ & $2.69 \times 10^{-4}$ & $5.96 \times 10^{-4}$ & 21 & 1.86 & $2.05 \times 10^{-3}$ & 1.86 \\
\hline ccy7.4 $1.26 \times 10^{-5}$ & $6.95 \times 10^{-4}$ & $1.92 \times 10^{-4}$ & $5.03 \times 10^{-4}$ & 15 & 1.32 & $1.73 \times 10^{-3}$ & 1.32 \\
\hline ccy7.5 $1.26 \times 10^{-5}$ & $6.05 \times 10^{-4}$ & $1.54 \times 10^{-4}$ & $4.51 \times 10^{-4}$ & 12 & 1.06 & $1.55 \times 10^{-3}$ & 1.06 \\
\hline ccy7.6 $1.26 \times 10^{-5}$ & $5.20 \times 10^{-4}$ & $1.21 \times 10^{-4}$ & $3.99 \times 10^{-4}$ & 10 & 0.816 & $1.37 \times 10^{-3}$ & 0.815 \\
\hline \multicolumn{8}{|c|}{$k_{2, \mathrm{~N}}=6.99 \times 10^{3} \mathrm{M}^{-1} \mathrm{~s}^{-1}$} \\
\hline \multicolumn{8}{|l|}{$k_{2, \mathrm{OH}^{-}}=3.44 \mathrm{M}^{-1} \mathrm{~s}^{-1}$} \\
\hline \multicolumn{8}{|l|}{$\mathrm{p} K_{\mathrm{B}}=2.88$} \\
\hline & & & & & & & \\
\hline & & & & & $\begin{array}{r}0.00 \\
{[\text { Nucle }}\end{array}$ & $\begin{array}{r}0.0010 \\
\text { hile } e_{\text {eff }} / \mathrm{mol} \mathrm{L}^{-1}\end{array}$ & 0.00 \\
\hline
\end{tabular}

26.1.6. Reaction of Piperidine (1x) with (lil) ${ }_{2} \mathrm{CH}^{+} \mathrm{BF}_{4}{ }^{-}$(at $20^{\circ} \mathrm{C}$, cosolvent: 0.4 vol- $\% \mathrm{CH}_{3} \mathrm{CN}$, stopped-flow, detection at $625 \mathrm{~nm}$ )

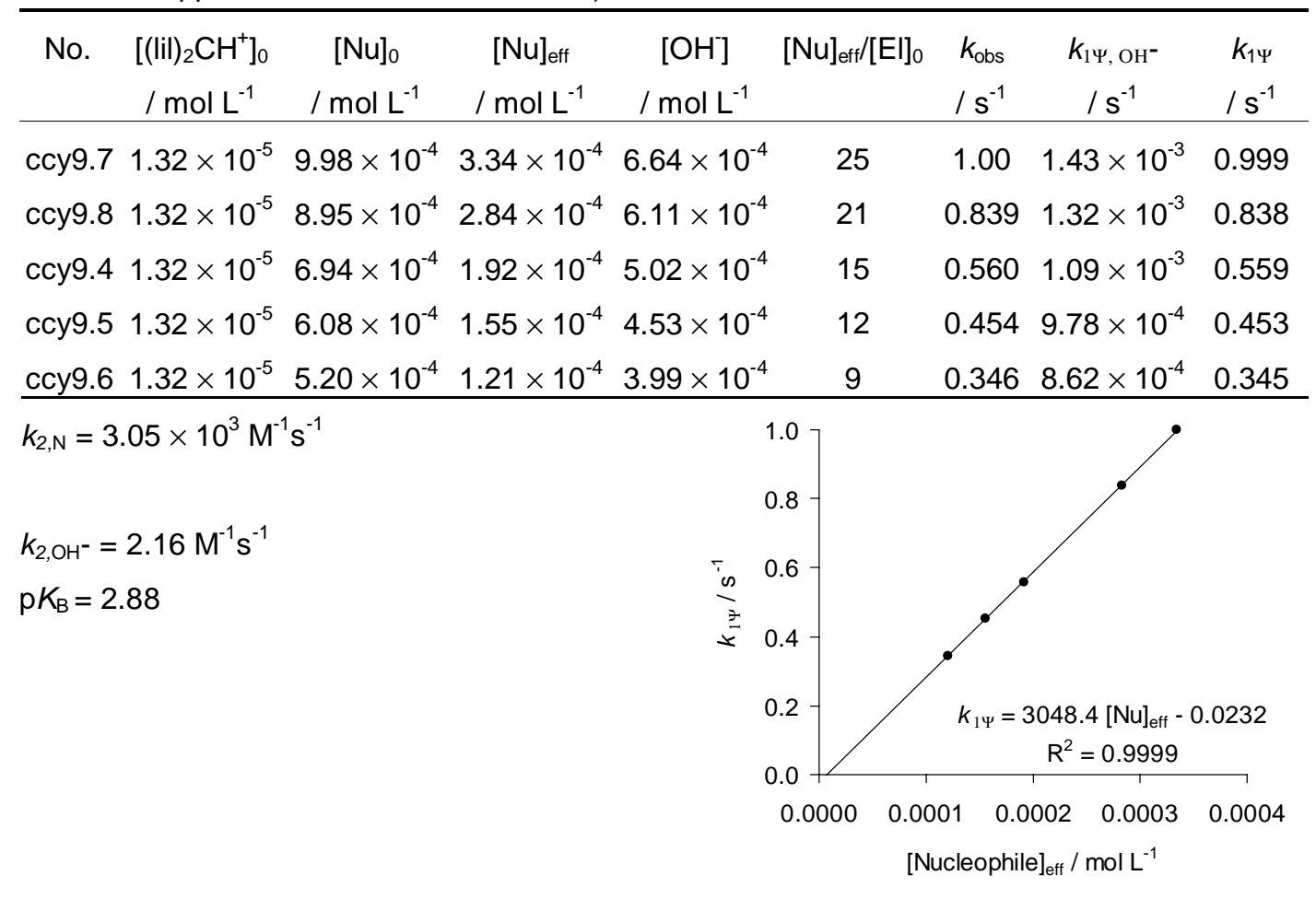


26.2. Reactivity parameters in water: $N=18.13 ; s=0.44$

\begin{tabular}{ccc}
\hline Reference electrophile & $E$ parameter & $k_{2, \mathrm{~N}}\left(20^{\circ} \mathrm{C}\right) / \mathrm{M}^{-1} \mathrm{~s}^{-1}$ \\
\hline$(\mathrm{dma})_{2} \mathrm{CH}^{+}$ & -7.02 & $6.09 \times 10^{4}$ \\
$(\text { pyr })_{2} \mathrm{CH}^{+}$ & -7.69 & $3.68 \times 10^{4}$ \\
$(\text { thq })_{2} \mathrm{CH}^{+}$ & -8.22 & $2.64 \times 10^{4}$ \\
$(\text { ind })_{2} \mathrm{CH}^{+}$ & -8.76 & $9.01 \times 10^{3}$ \\
$(\mathrm{jul})_{2} \mathrm{CH}^{+}$ & -9.45 & $6.99 \times 10^{3}$ \\
$(\mathrm{lil})_{2} \mathrm{CH}^{+}$ & -10.04 & $3.05 \times 10^{3}$ \\
\hline
\end{tabular}

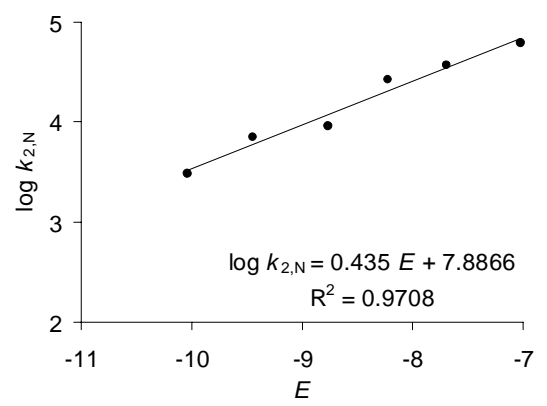

\section{Perhydroazepine (1y)}

27.1. Rate constants in water

27.1.1. Reaction of Perhydroazepine (1y) with $(\mathrm{dma}){ }_{2} \mathrm{CH}^{+} \mathrm{BF}_{4}{ }^{-}$(at $20^{\circ} \mathrm{C}$, cosolvent: 0.5 vol-\% $\mathrm{CH}_{3} \mathrm{CN}$, stopped-flow, detection at $610 \mathrm{~nm}$ )

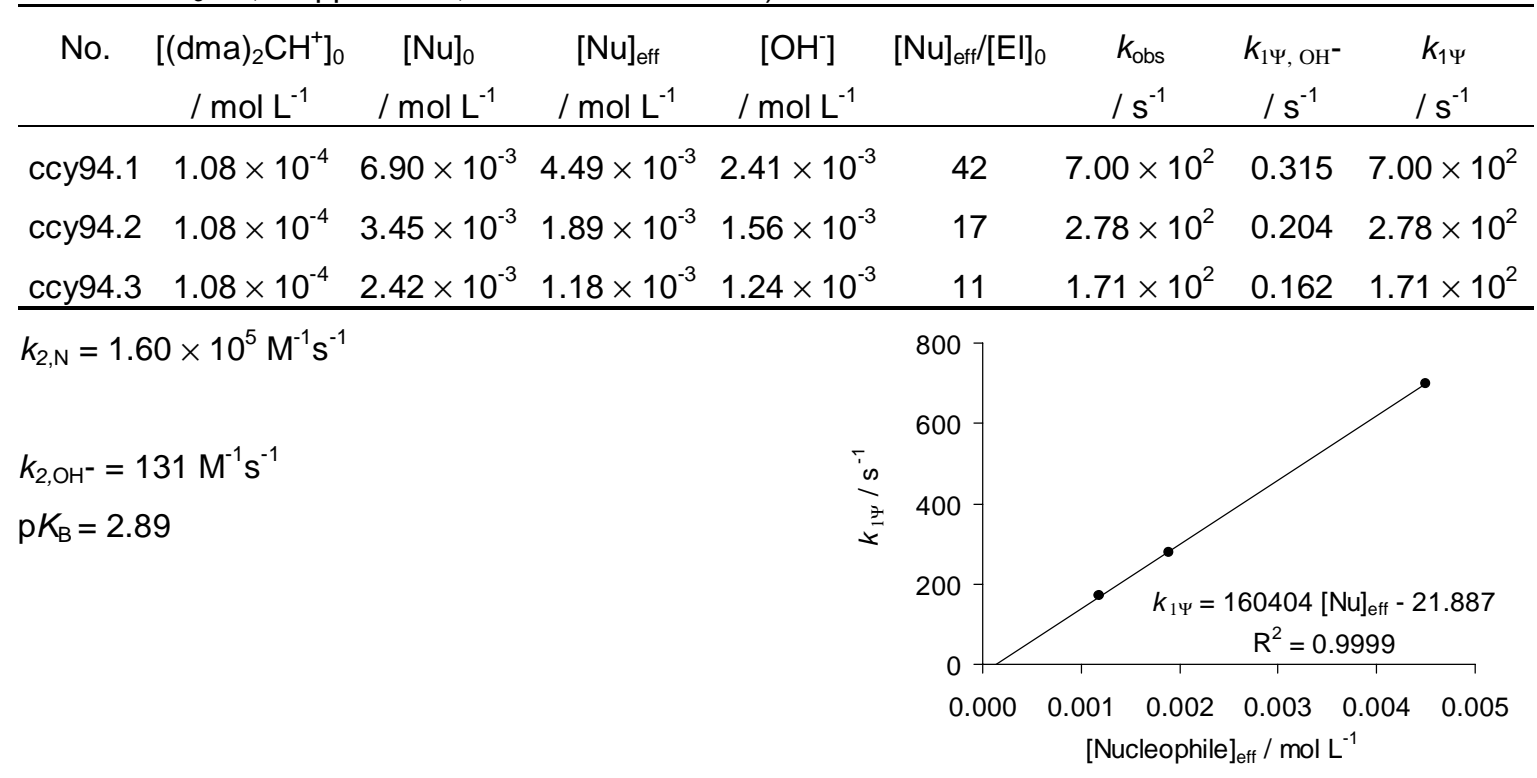


27.1.2. Reaction of Perhydroazepine (1y) with (pyr) ${ }_{2} \mathrm{CH}^{+} \mathrm{BF}_{4}{ }^{-}$(at $20^{\circ} \mathrm{C}$, cosolvent: 0.2 vol- $\% \mathrm{CH}_{3} \mathrm{CN}$, stopped-flow, detection at $615 \mathrm{~nm}$ )

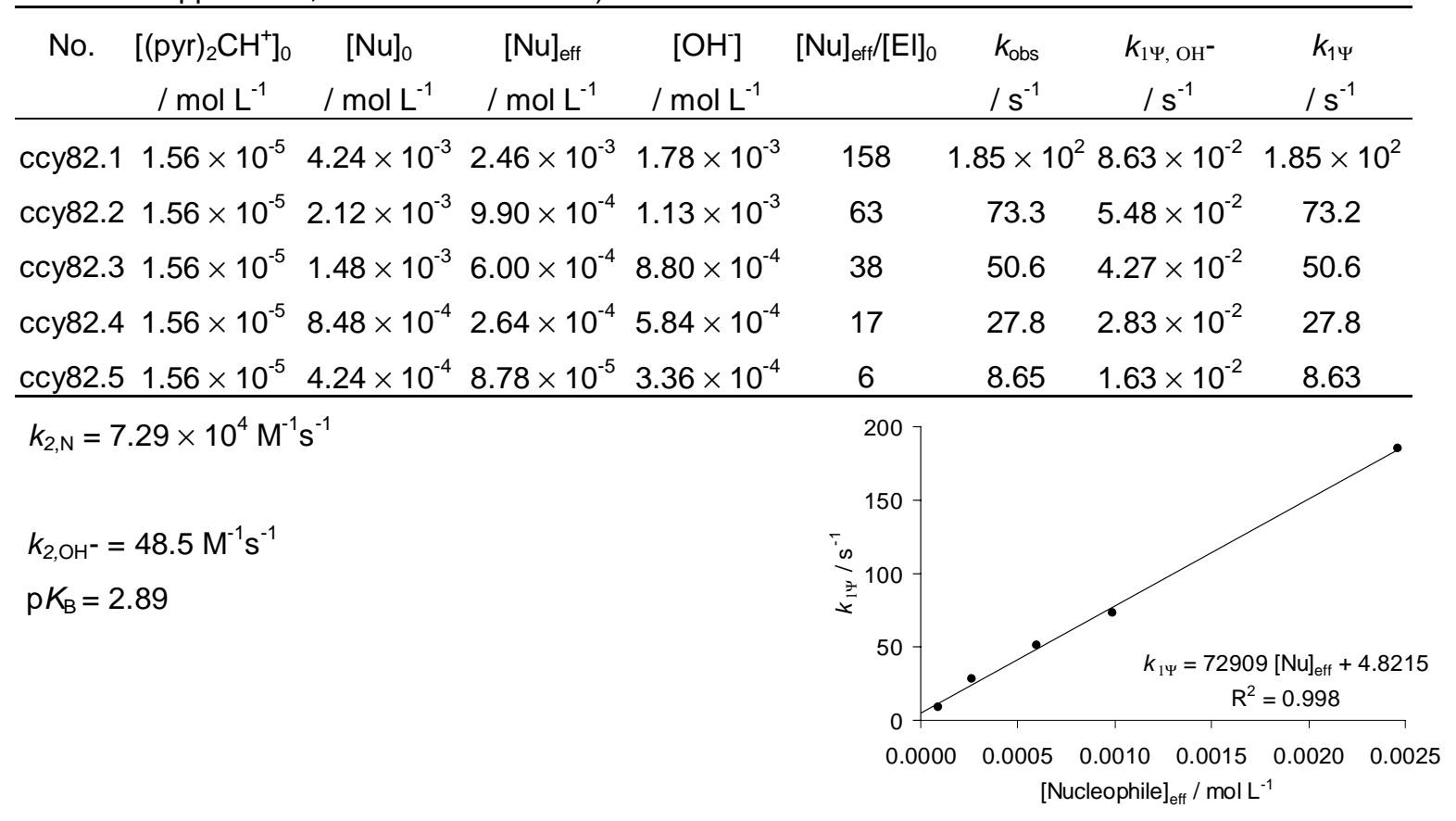

27.1.3. Reaction of Perhydroazepine (1y) with (thq) ${ }_{2} \mathrm{CH}^{+} \mathrm{BF}_{4}^{-}$(at $20^{\circ} \mathrm{C}$, cosolvent: 0.2 vol- $\% \mathrm{CH}_{3} \mathrm{CN}$, stopped-flow, detection at $610 \mathrm{~nm}$ )

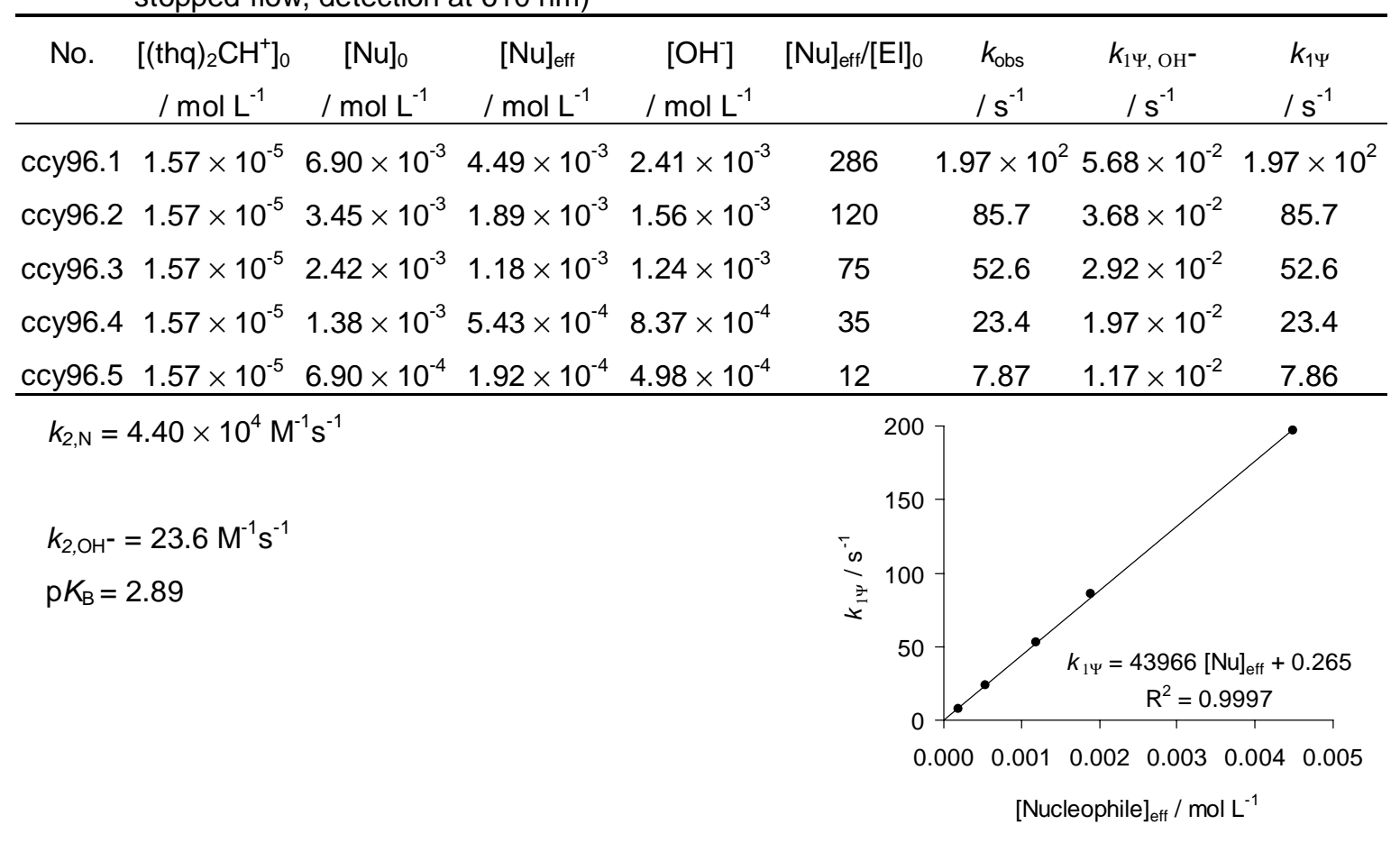


27.1.4. Reaction of Perhydroazepine (1y) with (ind) ${ }_{2} \mathrm{CH}^{+} \mathrm{BF}_{4}^{-}$(at $20^{\circ} \mathrm{C}$, cosolvent: 0.2 vol-\% $\mathrm{CH}_{3} \mathrm{CN}$, stopped-flow, detection at $615 \mathrm{~nm}$ )

\begin{tabular}{ccccccccc}
\hline No. & $\begin{array}{c}{\left[(\mathrm{ind})_{2} \mathrm{CH}^{+}\right]_{0}} \\
/ \mathrm{mol} \mathrm{L}^{-1}\end{array}$ & $\begin{array}{c}{[\mathrm{Nu}]_{0}} \\
/ \mathrm{mol} \mathrm{L}^{-1}\end{array}$ & $\begin{array}{c}{[\mathrm{Nu}]_{\mathrm{eff}}} \\
/ \mathrm{mol} \mathrm{L}^{-1}\end{array}$ & $\begin{array}{c}{[\mathrm{OH}]} \\
/ \mathrm{mol} \mathrm{L}^{-1}\end{array}$ & $\begin{array}{c}{[\mathrm{Nu}]_{\mathrm{eff}} /[\mathrm{El}]_{0}} \\
k_{\mathrm{obs}}\end{array}$ & $\begin{array}{c}k_{1 \Psi, \mathrm{OH}^{-}} \\
/ \mathrm{s}^{-1}\end{array}$ & $\begin{array}{c}k_{1 \Psi} \\
/ \mathrm{s}^{-1}\end{array}$ & $/ \mathrm{s}^{-1}$ \\
\hline ccy83.1 & $1.32 \times 10^{-5}$ & $4.24 \times 10^{-3}$ & $2.46 \times 10^{-3}$ & $1.78 \times 10^{-3}$ & 186 & 52.1 & $1.92 \times 10^{-2}$ & 52.1 \\
ccy83.2 & $1.32 \times 10^{-5}$ & $2.12 \times 10^{-3}$ & $9.90 \times 10^{-4}$ & $1.13 \times 10^{-3}$ & 75 & 20.5 & $1.22 \times 10^{-2}$ & 20.5 \\
fb203.1 & $2.34 \times 10^{-5}$ & $2.45 \times 10^{-3}$ & $1.20 \times 10^{-3}$ & $1.25 \times 10^{-3}$ & 51 & 24.9 & $1.35 \times 10^{-2}$ & 24.9 \\
ccy83.3 & $1.32 \times 10^{-5}$ & $1.48 \times 10^{-3}$ & $6.00 \times 10^{-4}$ & $8.80 \times 10^{-4}$ & 45 & 12.3 & $9.50 \times 10^{-3}$ & 12.3 \\
fb203.2 & $2.34 \times 10^{-5}$ & $1.72 \times 10^{-3}$ & $7.42 \times 10^{-4}$ & $9.78 \times 10^{-4}$ & 32 & 15.0 & $1.06 \times 10^{-2}$ & 15.0 \\
fb203.3 & $2.34 \times 10^{-5}$ & $1.23 \times 10^{-3}$ & $4.60 \times 10^{-4}$ & $7.70 \times 10^{-4}$ & 20 & 9.91 & $8.31 \times 10^{-3}$ & 9.90 \\
fb203.3b & $2.34 \times 10^{-5}$ & $1.23 \times 10^{-3}$ & $4.60 \times 10^{-4}$ & $7.70 \times 10^{-4}$ & 20 & 9.04 & $8.31 \times 10^{-3}$ & 9.03 \\
ccy83.4 & $1.32 \times 10^{-5}$ & $8.48 \times 10^{-4}$ & $2.64 \times 10^{-4}$ & $5.84 \times 10^{-4}$ & 20 & 5.27 & $6.30 \times 10^{-3}$ & 5.26 \\
fb203.4b & $2.34 \times 10^{-5}$ & $7.35 \times 10^{-4}$ & $2.12 \times 10^{-4}$ & $5.23 \times 10^{-4}$ & 9 & 4.01 & $5.65 \times 10^{-3}$ & 4.00 \\
ccy83.5 & $1.32 \times 10^{-5}$ & $4.24 \times 10^{-4}$ & $8.78 \times 10^{-5}$ & $3.36 \times 10^{-4}$ & 7 & 1.63 & $3.63 \times 10^{-3}$ & 1.63 \\
fb203.5 & $2.34 \times 10^{-5}$ & $4.90 \times 10^{-4}$ & $1.11 \times 10^{-4}$ & $3.79 \times 10^{-4}$ & 5 & 1.92 & $4.09 \times 10^{-3}$ & 1.92 \\
\hline
\end{tabular}

$k_{2, \mathrm{~N}}=2.13 \times 10^{4} \mathrm{M}^{-1} \mathrm{~s}^{-1}$

$k_{2, \mathrm{OH}}=10.8 \mathrm{M}^{-1} \mathrm{~s}^{-1}$

$\mathrm{p} K_{\mathrm{B}}=2.89$

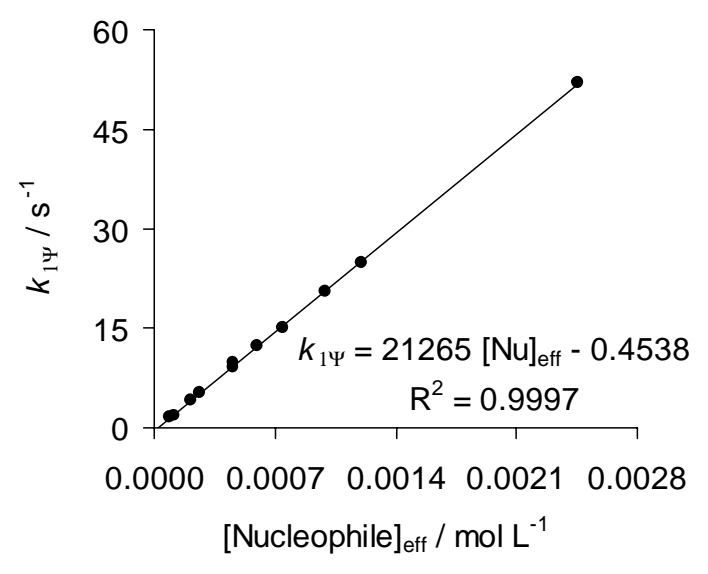


27.1.5. Reaction of Perhydroazepine (1y) with $(\mathrm{jul})_{2} \mathrm{CH}^{+} \mathrm{BF}_{4}{ }^{-}$(at $20^{\circ} \mathrm{C}$, cosolvent: 0.2 vol- $\% \mathrm{CH}_{3} \mathrm{CN}$, stopped-flow, detection at $630 \mathrm{~nm}$ )

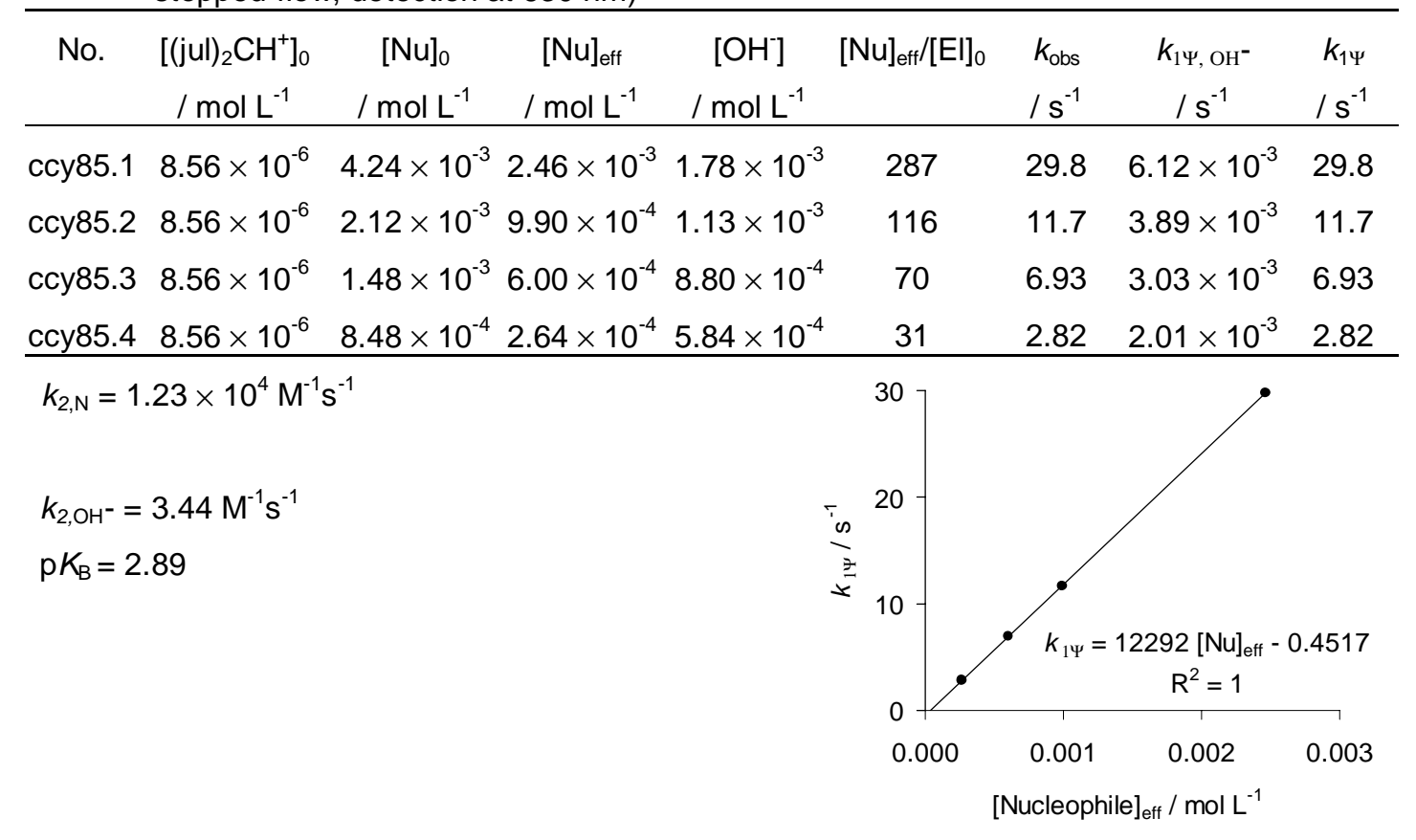

27.1.6. Reaction of Perhydroazepine (1y) with (lil) ${ }_{2} \mathrm{CH}^{+} \mathrm{BF}_{4}^{-}$(at $20{ }^{\circ} \mathrm{C}$, cosolvent: 0.4 vol-\% $\mathrm{CH}_{3} \mathrm{CN}$, stopped-flow, detection at $630 \mathrm{~nm}$ )

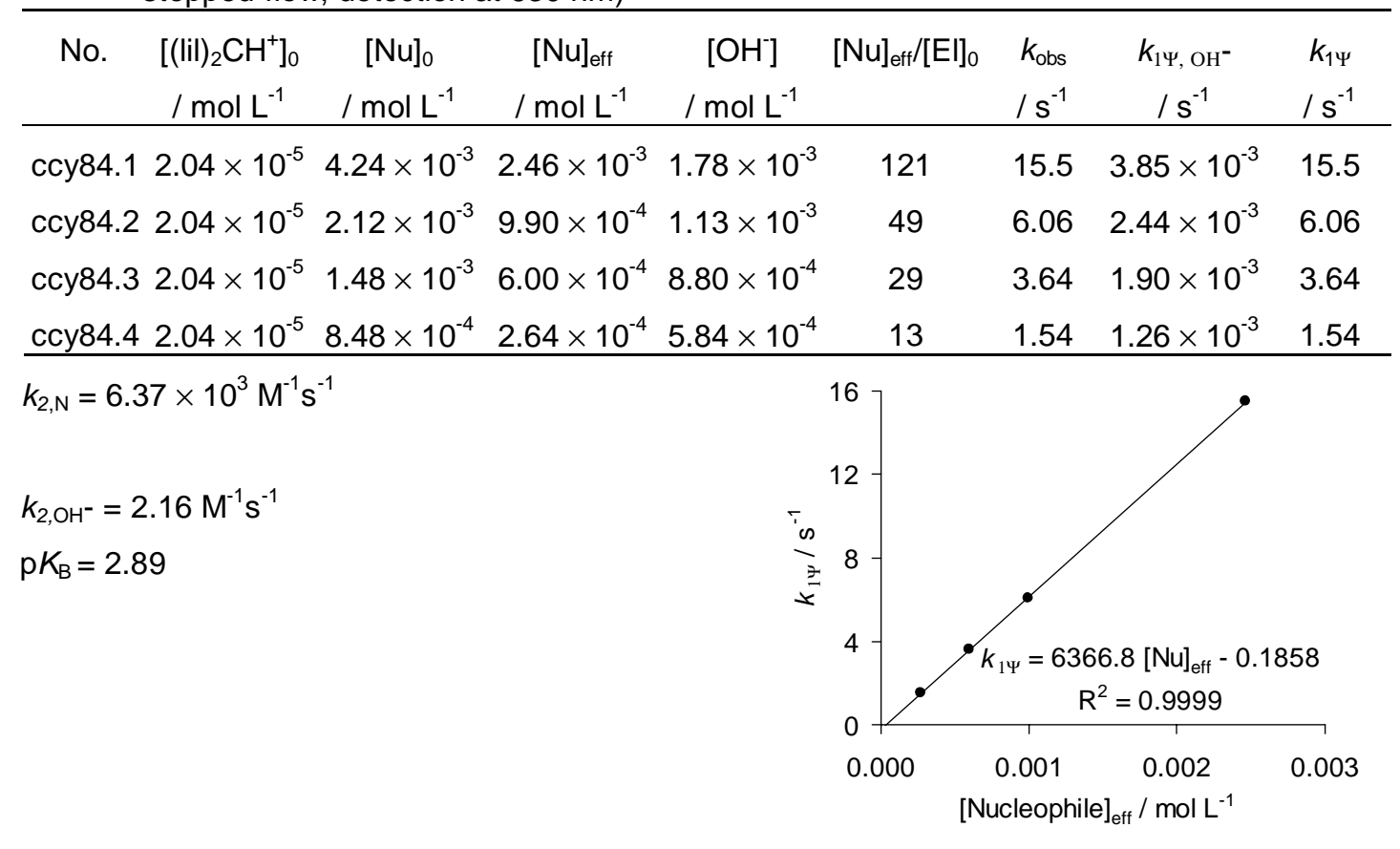


27.2. Reactivity parameters in water: $N=18.29 ; s=0.46$

\begin{tabular}{ccc}
\hline Reference electrophile & $E$ parameter & $k_{2, \mathrm{~N}}\left(20^{\circ} \mathrm{C}\right) / \mathrm{M}^{-1} \mathrm{~s}^{-1}$ \\
\hline$(\mathrm{dma})_{2} \mathrm{CH}^{+}$ & -7.02 & $1.60 \times 10^{5}$ \\
$(\text { pyr })_{2} \mathrm{CH}^{+}$ & -7.69 & $7.29 \times 10^{4}$ \\
$(\text { thq })_{2} \mathrm{CH}^{+}$ & -8.22 & $4.40 \times 10^{4}$ \\
$(\text { ind })_{2} \mathrm{CH}^{+}$ & -8.76 & $2.13 \times 10^{4}$ \\
$(\mathrm{jul}){ }_{2} \mathrm{CH}^{+}$ & -9.45 & $1.23 \times 10^{4}$ \\
$(\mathrm{lil})_{2} \mathrm{CH}^{+}$ & -10.04 & $6.37 \times 10^{3}$ \\
\hline
\end{tabular}

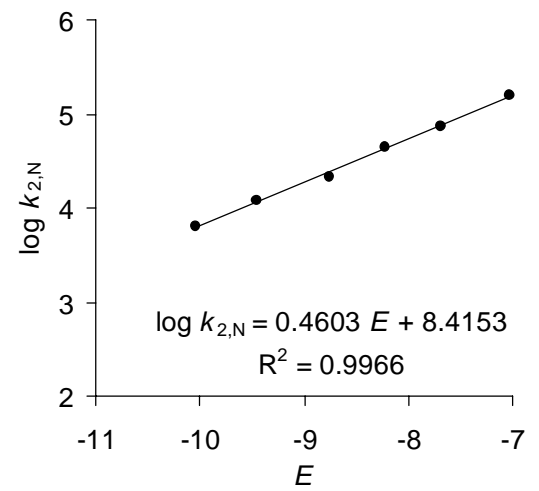

\section{Morpholine (1z)}

28.1. Rate constants in water

28.1.1. Reaction of Morpholine (1z) with (mor) ${ }_{2} \mathrm{CH}^{+} \mathrm{BF}_{4}^{-}$(at $20^{\circ} \mathrm{C}$, cosolvent 9 vol- $\% \mathrm{CH}_{3} \mathrm{CN}$, stopped-flow, detection at $605 \mathrm{~nm}$ )

\begin{tabular}{ccccccccc}
\hline No. & $\begin{array}{c}{\left[(\mathrm{mor})_{2} \mathrm{CH}^{+}\right]_{0}} \\
/ \mathrm{mol} \mathrm{L}^{-1}\end{array}$ & $\begin{array}{c}{[\mathrm{Nu}]_{0}} \\
/ \mathrm{mol} \mathrm{L}^{-1}\end{array}$ & $\begin{array}{c}{[\mathrm{Nu}]_{\mathrm{eff}}} \\
/ \mathrm{mol} \mathrm{L}^{-1}\end{array}$ & $\begin{array}{c}{[\mathrm{OH}]} \\
/ \mathrm{mol} \mathrm{L}^{-1}\end{array}$ & $\begin{array}{c}{[\mathrm{Nu}]_{\mathrm{eff}} /[\mathrm{El}]_{0}} \\
k_{\mathrm{obs}} \\
/ \mathrm{s}^{-1}\end{array}$ & $\begin{array}{c}k_{1 \Psi, \mathrm{OH}^{-}} \\
/ \mathrm{s}^{-1}\end{array}$ & $\begin{array}{c}k_{1 \Psi} \\
/ \mathrm{s}^{-1}\end{array}$ \\
\hline ccy14.1 & $7.28 \times 10^{-6}$ & $2.63 \times 10^{-3}$ & $2.55 \times 10^{-3}$ & $7.65 \times 10^{-5}$ & 351 & $7.96 \times 10^{2}$ & $8.11 \times 10^{-2}$ & $7.96 \times 10^{2}$ \\
ccy14.2 & $7.28 \times 10^{-6}$ & $1.76 \times 10^{-3}$ & $1.70 \times 10^{-3}$ & $6.24 \times 10^{-5}$ & 233 & $5.42 \times 10^{2}$ & $6.61 \times 10^{-2}$ & $5.42 \times 10^{2}$ \\
ccy14.3 & $7.28 \times 10^{-6}$ & $8.78 \times 10^{-4}$ & $8.34 \times 10^{-4}$ & $4.37 \times 10^{-5}$ & 115 & $2.56 \times 10^{2}$ & $4.63 \times 10^{-2}$ & $2.56 \times 10^{2}$ \\
ccy14.4 & $7.28 \times 10^{-6}$ & $5.27 \times 10^{-4}$ & $4.93 \times 10^{-4}$ & $3.36 \times 10^{-5}$ & 68 & $1.51 \times 10^{2}$ & $3.56 \times 10^{-2}$ & $1.51 \times 10^{2}$ \\
ccy14.5 $7.28 \times 10^{-6}$ & $3.51 \times 10^{-4}$ & $3.24 \times 10^{-4}$ & $2.72 \times 10^{-5}$ & 44 & 97.2 & $2.89 \times 10^{-2}$ & 97.2 \\
ccy14.6 $7.28 \times 10^{-6}$ & $1.76 \times 10^{-4}$ & $1.57 \times 10^{-4}$ & $1.90 \times 10^{-5}$ & 22 & 41.1 & $2.01 \times 10^{-2}$ & 41.1 \\
\hline
\end{tabular}

$k_{2, \mathrm{~N}}=3.16 \times 10^{5} \mathrm{M}^{-1} \mathrm{~s}^{-1}$

$k_{2, \mathrm{OH}^{-}}=1060 \mathrm{M}^{-1} \mathrm{~s}^{-1}$

$\mathrm{p} K_{\mathrm{B}}=5.64$

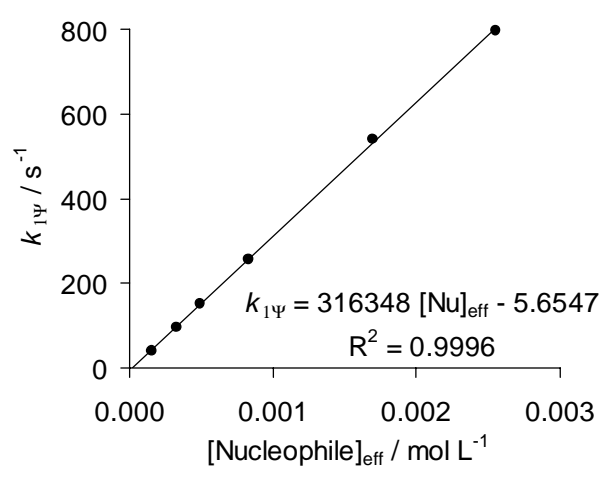


28.1.2. Reaction of Morpholine (1z) with $(\mathrm{dma}){ }_{2} \mathrm{CH}^{+} \mathrm{BF}_{4}{ }^{-}$(at $20^{\circ} \mathrm{C}$, cosolvent 0.5 vol- $\% \mathrm{CH}_{3} \mathrm{CN}$, stopped-flow, detection at $605 \mathrm{~nm}$ )

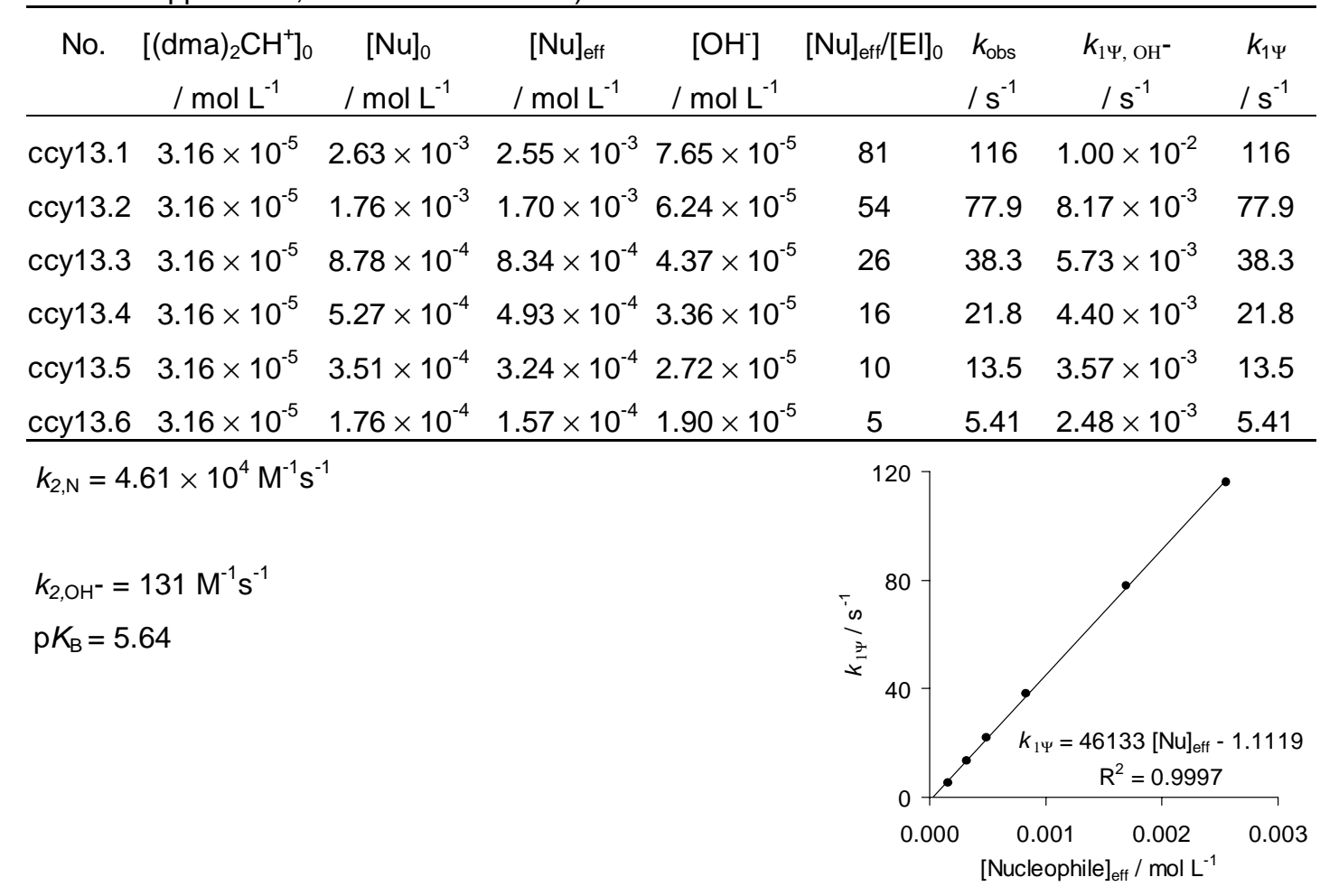

28.1.3. Reaction of Morpholine (1z) with (pyr) ${ }_{2} \mathrm{CH}^{+} \mathrm{BF}_{4}^{-}$(at $20^{\circ} \mathrm{C}$, cosolvent 9 vol-\% $\mathrm{CH}_{3} \mathrm{CN}$, stopped-flow, detection at $614 \mathrm{~nm}$ )

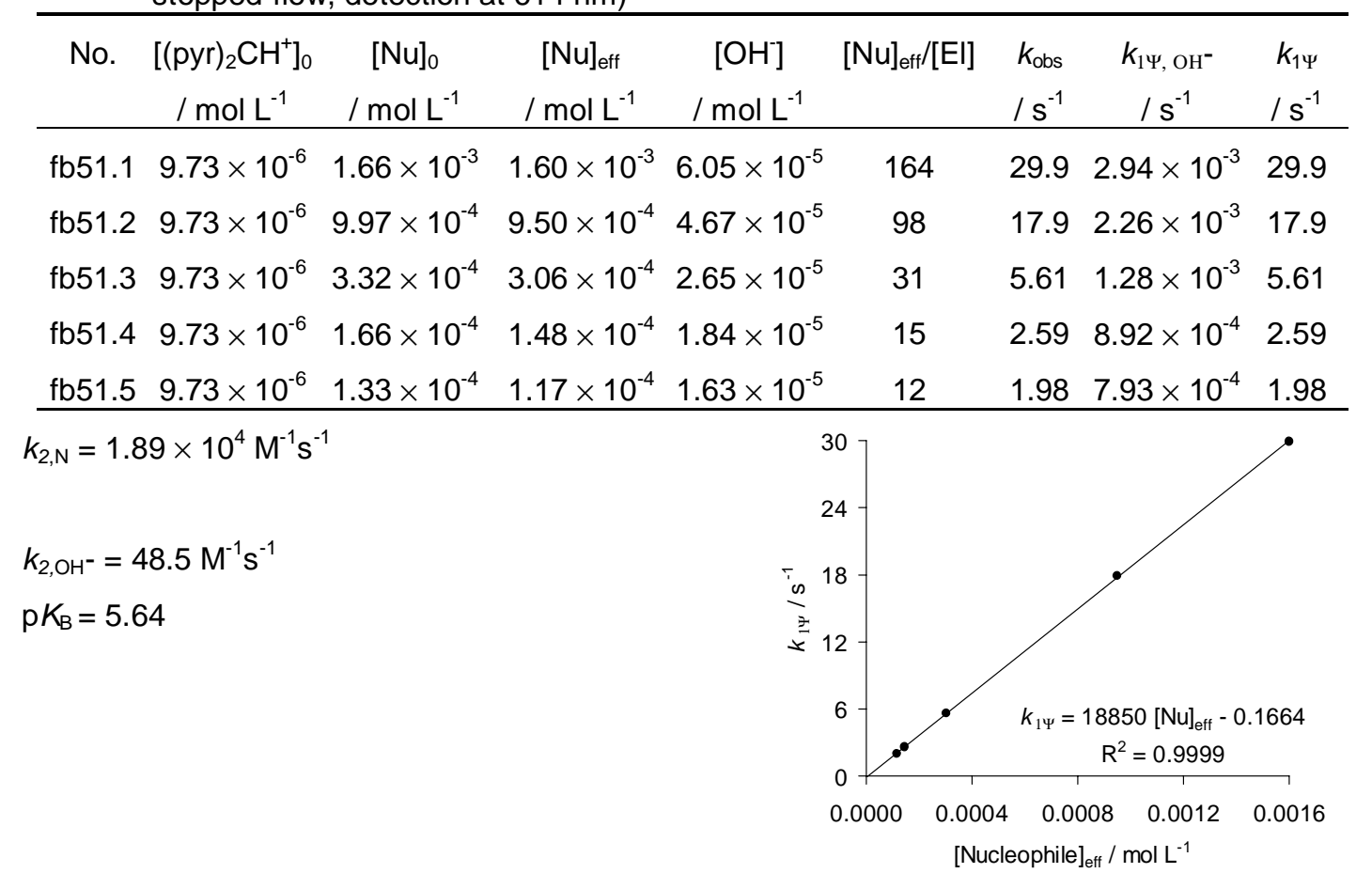


28.1.4. Reaction of Morpholine (1z) with (thq) ${ }_{2} \mathrm{CH}^{+} \mathrm{BF}_{4}{ }^{-}$(at $20^{\circ} \mathrm{C}$, cosolvent 0.4 vol- $\% \mathrm{CH}_{3} \mathrm{CN}$, stopped-flow, detection at $618 \mathrm{~nm}$ )

\begin{tabular}{ccccccccc}
\hline No. & $\begin{array}{c}{\left[(\text { thq })_{2} \mathrm{CH}^{+}\right]_{0}} \\
/ \mathrm{mol} \mathrm{L}^{-1}\end{array}$ & $\begin{array}{c}{[\mathrm{Nu}]_{0}} \\
/ \mathrm{mol} \mathrm{L}^{-1}\end{array}$ & $\begin{array}{c}{[\mathrm{Nu}]_{\text {eff }}} \\
/ \mathrm{mol} \mathrm{L}^{-1}\end{array}$ & $\begin{array}{c}{[\mathrm{OH}]} \\
/ \mathrm{mol} \mathrm{L}^{-1}\end{array}$ & $\begin{array}{c}{[\mathrm{Nu}]_{\mathrm{eff} f} /[\mathrm{El}]_{0}} \\
k_{\mathrm{obs}}\end{array}$ & $\begin{array}{c}k_{1 \Psi} \mathrm{OH}^{-} \\
/ \mathrm{s}^{-1}\end{array}$ & $\begin{array}{c}k_{1 \Psi} \\
/ \mathrm{s}^{-1}\end{array}$ & $/ \mathrm{s}^{-1}$ \\
\hline fb57.1 & $1.86 \times 10^{-5}$ & $3.32 \times 10^{-3}$ & $3.23 \times 10^{-3}$ & $8.61 \times 10^{-5}$ & 174 & 34.7 & $2.03 \times 10^{-3}$ & 34.7 \\
fb58.2 & $1.86 \times 10^{-5}$ & $1.66 \times 10^{-3}$ & $1.60 \times 10^{-3}$ & $6.05 \times 10^{-5}$ & 86 & 17.1 & $1.43 \times 10^{-3}$ & 17.1 \\
fb57.2 & $1.86 \times 10^{-5}$ & $1.66 \times 10^{-3}$ & $1.60 \times 10^{-3}$ & $6.05 \times 10^{-5}$ & 86 & 17.0 & $1.43 \times 10^{-3}$ & 17.0 \\
fb58.3 & $1.86 \times 10^{-5}$ & $9.97 \times 10^{-4}$ & $9.50 \times 10^{-4}$ & $4.67 \times 10^{-5}$ & 51 & 9.88 & $1.10 \times 10^{-3}$ & 9.88 \\
fb57.3 & $1.86 \times 10^{-5}$ & $9.97 \times 10^{-4}$ & $9.50 \times 10^{-4}$ & $4.67 \times 10^{-5}$ & 51 & 9.78 & $1.10 \times 10^{-3}$ & 9.78 \\
fb53.1 & $5.36 \times 10^{-5}$ & $1.66 \times 10^{-3}$ & $1.60 \times 10^{-3}$ & $6.05 \times 10^{-5}$ & 30 & 16.2 & $1.43 \times 10^{-3}$ & 16.2 \\
fb53.2 & $5.36 \times 10^{-5}$ & $9.97 \times 10^{-4}$ & $9.50 \times 10^{-4}$ & $4.67 \times 10^{-5}$ & 18 & 9.15 & $1.10 \times 10^{-3}$ & 9.15 \\
fb58.4 & $1.86 \times 10^{-5}$ & $3.32 \times 10^{-4}$ & $3.06 \times 10^{-4}$ & $2.65 \times 10^{-5}$ & 16 & 2.97 & $6.24 \times 10^{-4}$ & 2.97 \\
fb57.4 & $1.86 \times 10^{-5}$ & $3.32 \times 10^{-4}$ & $3.06 \times 10^{-4}$ & $2.65 \times 10^{-5}$ & 16 & 3.05 & $6.24 \times 10^{-4}$ & 3.05 \\
\hline
\end{tabular}

$k_{2, \mathrm{~N}}=1.08 \times 10^{4} \mathrm{M}^{-1} \mathrm{~s}^{-1}$

$k_{2, \mathrm{OH}^{-}}=23.6 \mathrm{M}^{-1} \mathrm{~s}^{-1}$

$\mathrm{p} K_{\mathrm{B}}=5.64$

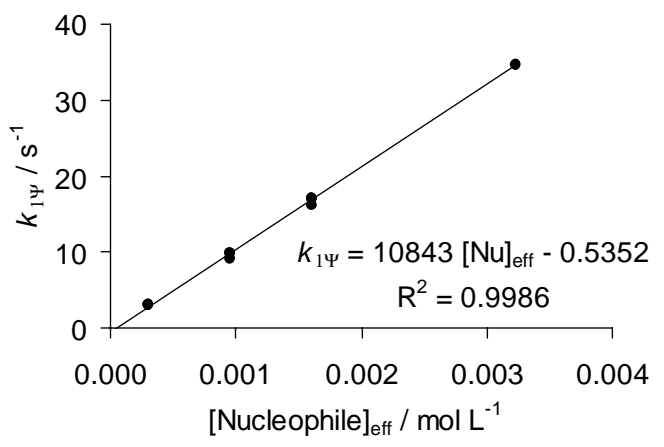


28.1.5. Reaction of Morpholine (1z) with (ind) ${ }_{2} \mathrm{CH}^{+} \mathrm{BF}_{4}{ }_{4}$ (at $20^{\circ} \mathrm{C}$, cosolvent 0.4 vol- $\% \mathrm{CH}_{3} \mathrm{CN}$, stopped-flow, detection at $614 \mathrm{~nm}$ )

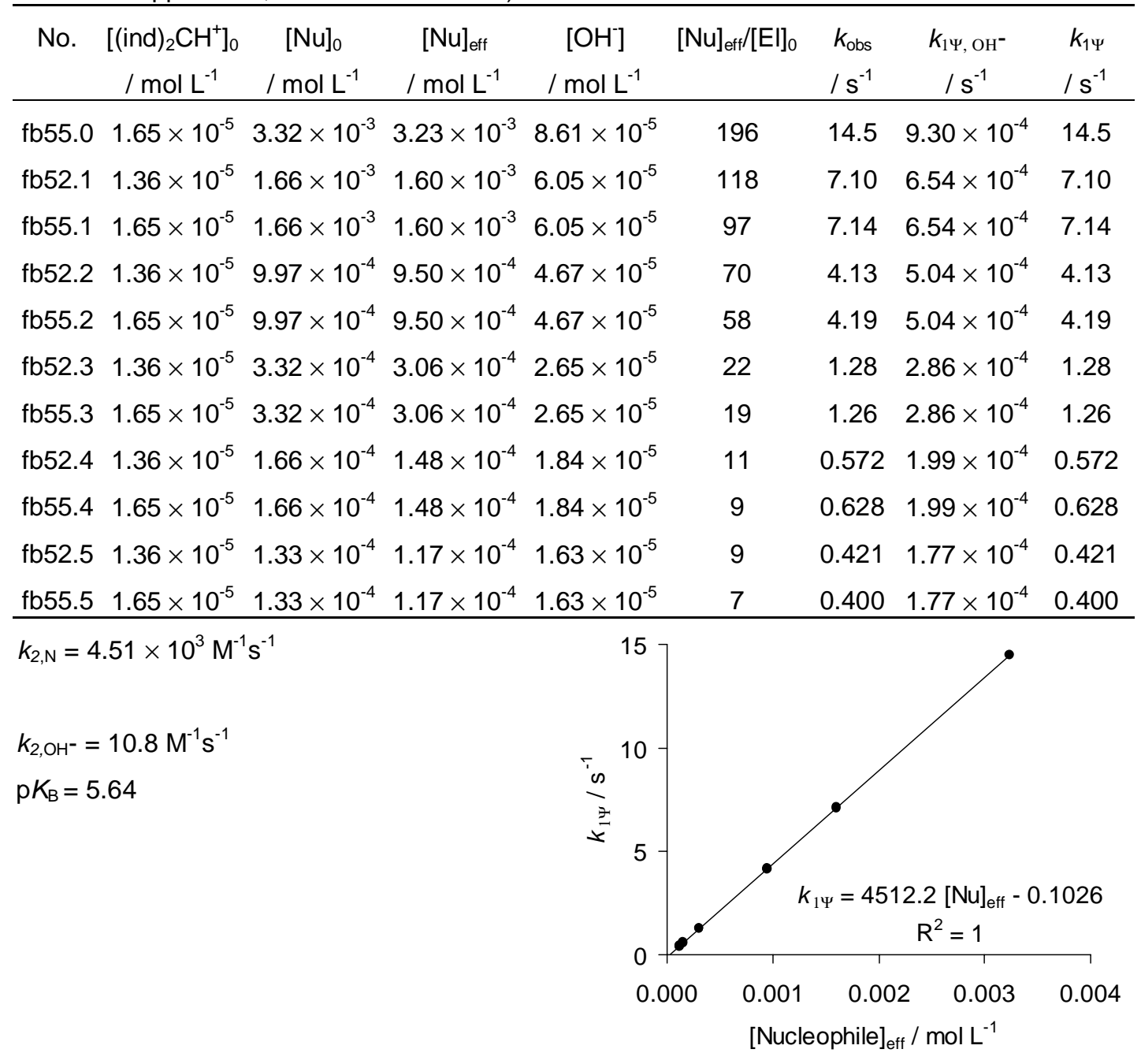


28.1.6. Reaction of Morpholine (1z) with $(\mathrm{jul})_{2} \mathrm{CH}^{+} \mathrm{BF}_{4}{ }^{-}$(at $20^{\circ} \mathrm{C}$, cosolvent 0.4 vol- $\% \mathrm{CH}_{3} \mathrm{CN}$, stopped-flow, detection at $634 \mathrm{~nm}$ )

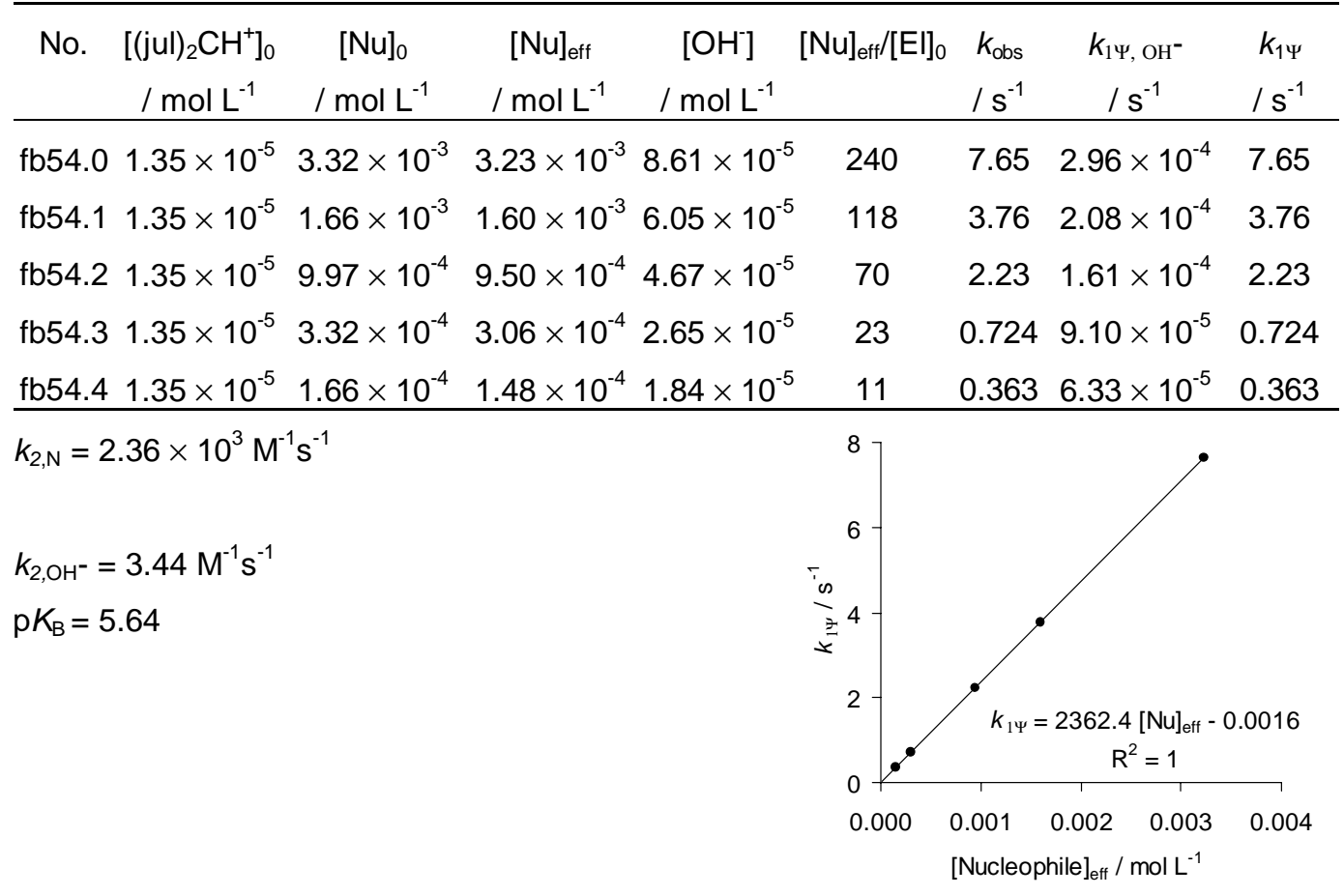

28.1.7. Reaction of Morpholine (1z) with (lil) ${ }_{2} \mathrm{CH}^{+} \mathrm{BF}_{4}^{-}$(at $20^{\circ} \mathrm{C}$, cosolvent 0.2 vol-\% $\mathrm{CH}_{3} \mathrm{CN}$, stopped-flow, detection at $630 \mathrm{~nm}$ )

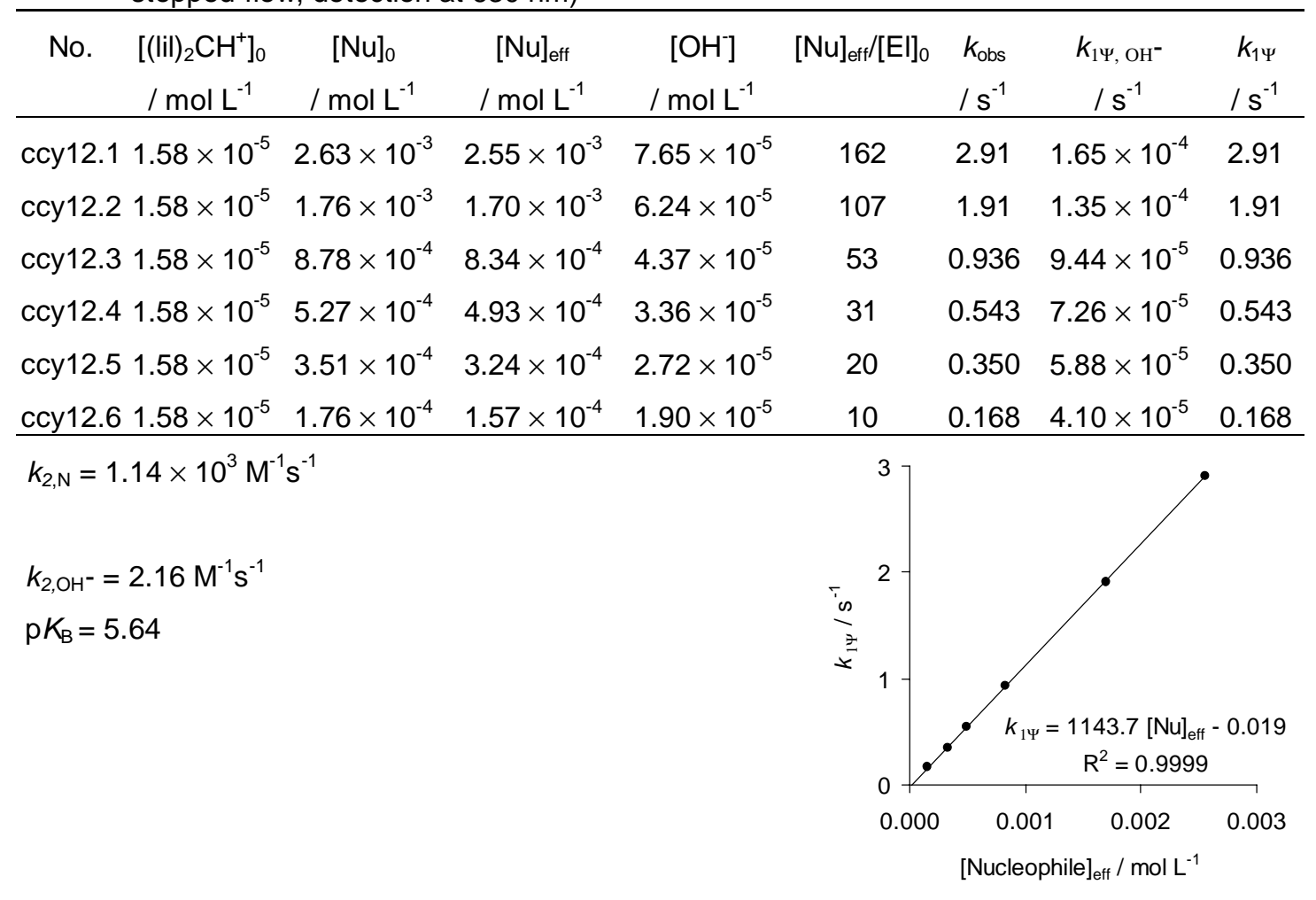


28.2. Reactivity parameters in water: $N=15.62 ; s=0.54$

\begin{tabular}{ccc}
\hline Reference electrophile & $E$ parameter & $k_{2, \mathrm{~N}}\left(20^{\circ} \mathrm{C}\right) / \mathrm{M}^{-1} \mathrm{~s}^{-1}$ \\
\hline (mor $)_{2} \mathrm{CH}^{+}$ & -5.53 & $3.16 \times 10^{5}$ \\
$(\mathrm{dma})_{2} \mathrm{CH}^{+}$ & -7.02 & $4.61 \times 10^{4}$ \\
$(\text { pyr })_{2} \mathrm{CH}^{+}$ & -7.69 & $1.89 \times 10^{4}$ \\
$(\text { thq })_{2} \mathrm{CH}^{+}$ & -8.22 & $1.08 \times 10^{4}$ \\
$(\text { ind })_{2} \mathrm{CH}^{+}$ & -8.76 & $4.51 \times 10^{3}$ \\
$(\mathrm{jul})_{2} \mathrm{CH}^{+}$ & -9.45 & $2.36 \times 10^{3}$ \\
$(\mathrm{lil})_{2} \mathrm{CH}^{+}$ & -10.04 & $1.14 \times 10^{3}$ \\
\hline
\end{tabular}

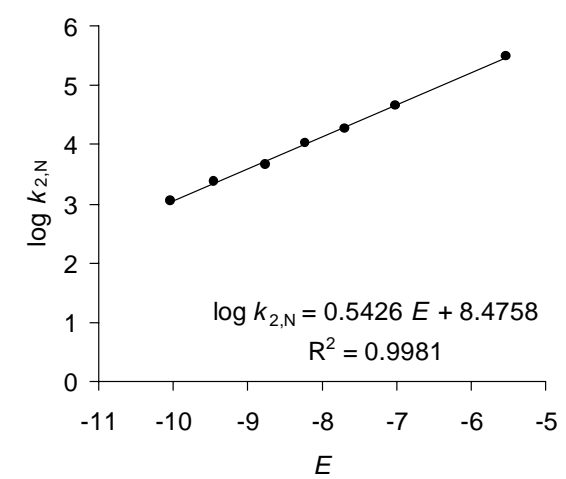

\section{Piperazine (1zz)}

29.1. Rate constants in water

29.1.1. Reaction of Piperazine (1 $\mathbf{z z}$ ) with (mor) ${ }_{2} \mathrm{CH}^{+} \mathrm{BF}_{4}^{-}$(at $20^{\circ} \mathrm{C}$, cosolvent 9 vol-\% $\mathrm{CH}_{3} \mathrm{CN}$, stopped-flow, detection at $607 \mathrm{~nm}$ )

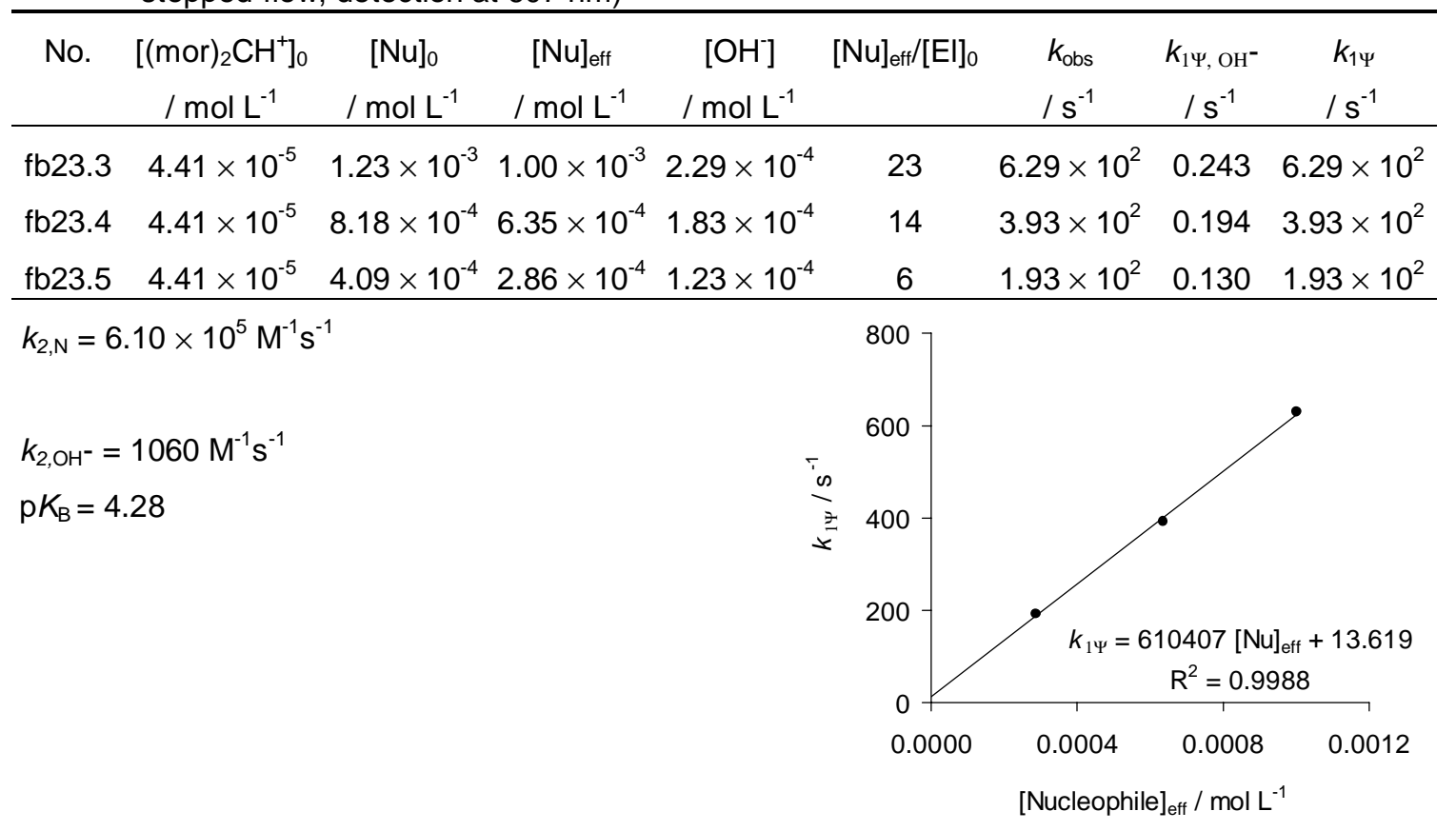


29.1.2. Reaction of Piperazine (1zz) with (dma) ${ }_{2} \mathrm{CH}^{+} \mathrm{BF}_{4}{ }_{4}^{-}$(at $20^{\circ} \mathrm{C}$, cosolvent 0.4 vol- $\% \mathrm{CH}_{3} \mathrm{CN}$, stopped-flow, detection at $604 \mathrm{~nm}$ )

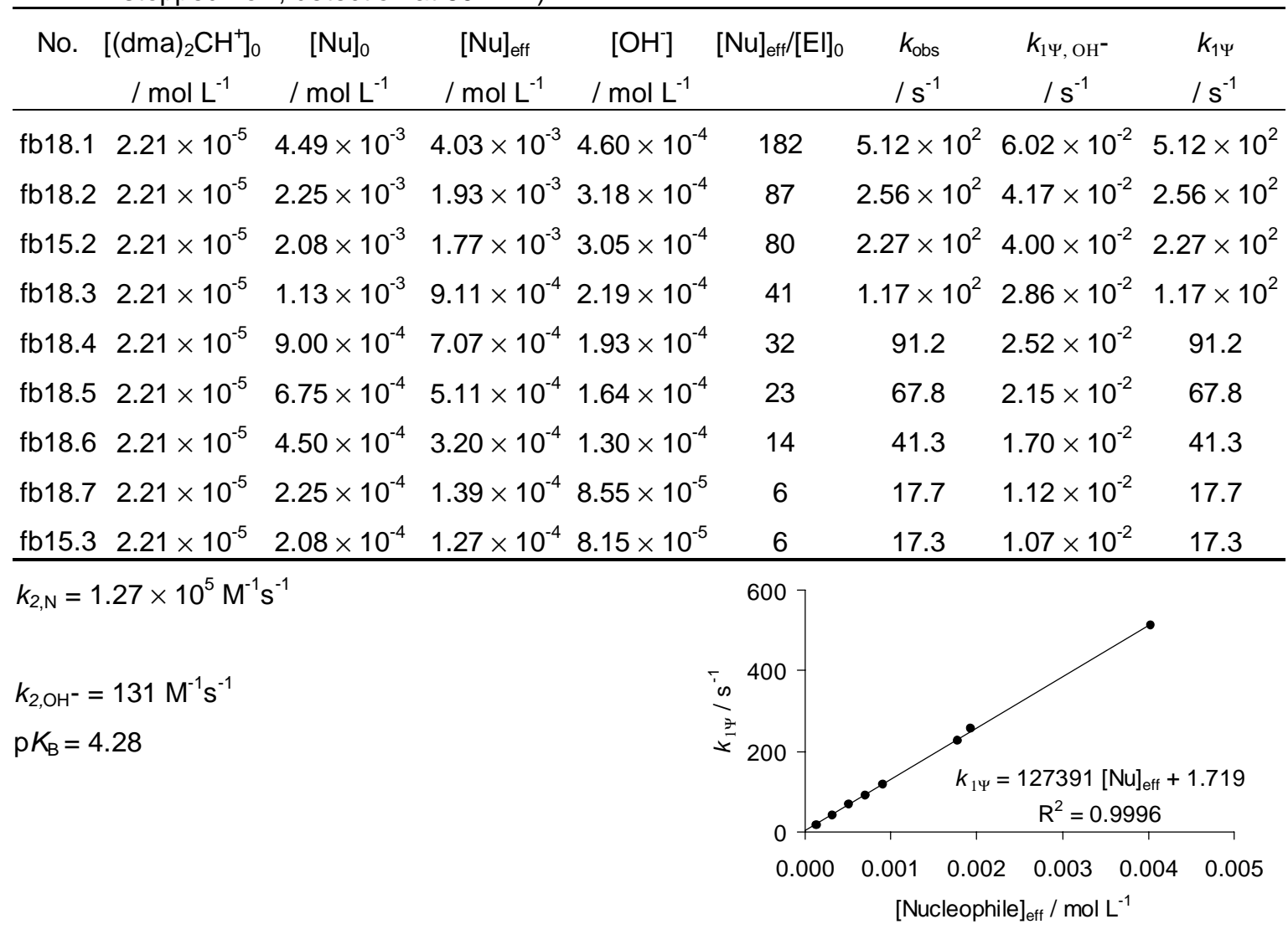

29.1.3. Reaction of Piperazine (1zz) with (pyr) ${ }_{2} \mathrm{CH}^{+} \mathrm{BF}_{4}^{-}$(at $20^{\circ} \mathrm{C}$, cosolvent 0.9 vol- $\% \mathrm{CH}_{3} \mathrm{CN}$, stopped-flow, detection at $611 \mathrm{~nm}$ )

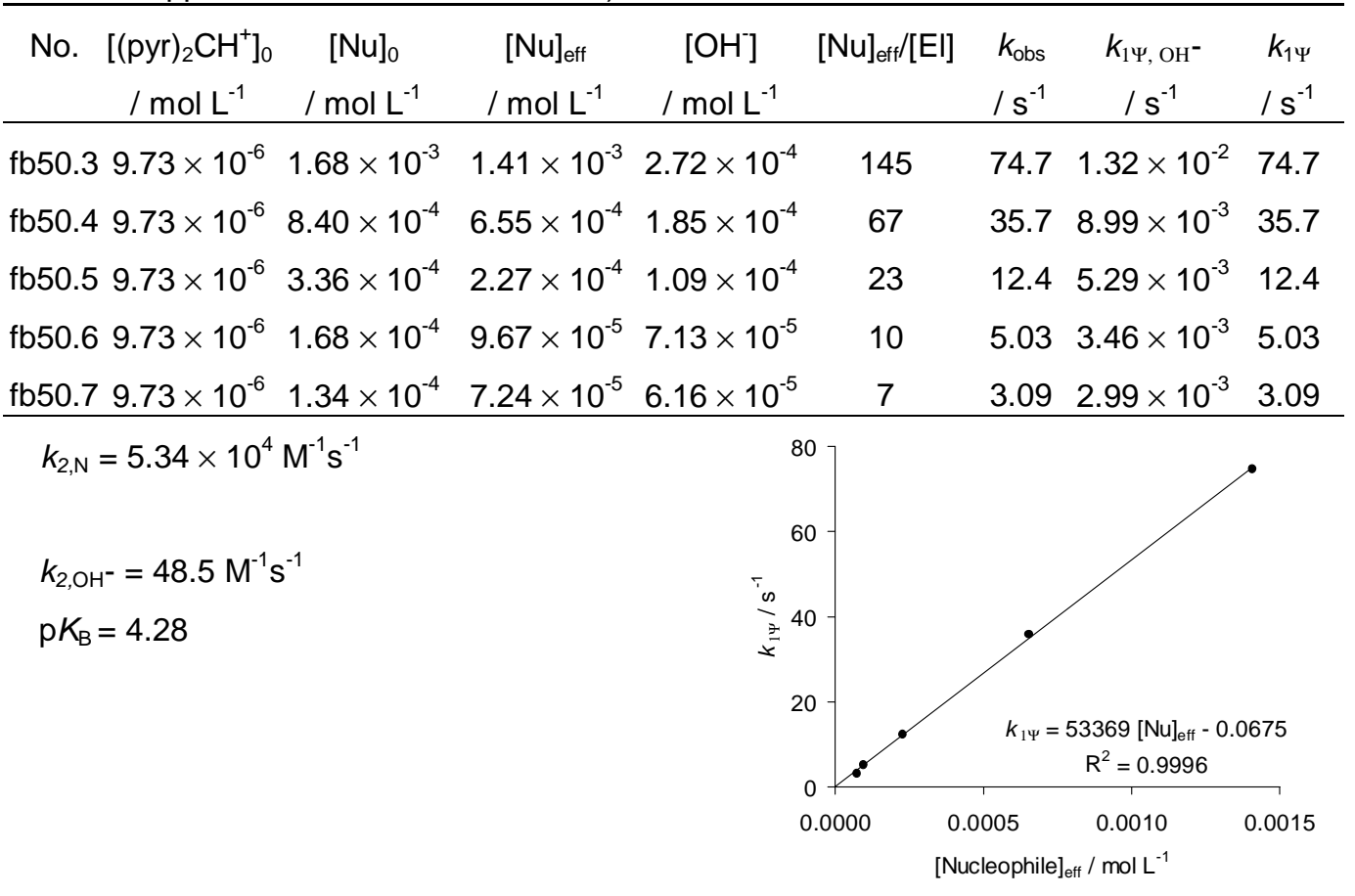


29.1.4. Reaction of Piperazine (1 $\mathbf{z z}$ ) with (thq) ${ }_{2} \mathrm{CH}^{+} \mathrm{BF}_{4}^{-}$(at $20{ }^{\circ} \mathrm{C}$, cosolvent 0.4 vol- $\% \mathrm{CH}_{3} \mathrm{CN}$, stopped-flow, detection at $618 \mathrm{~nm}$ )

\begin{tabular}{|c|c|c|c|c|c|c|c|c|}
\hline No. & $\begin{array}{c}{\left[(\text { thq })_{2} \mathrm{CH}^{+}\right]_{0}} \\
/ \mathrm{mol} \mathrm{L}^{-1}\end{array}$ & $\begin{array}{c}{[\mathrm{Nu}]_{0}} \\
/ \mathrm{mol} \mathrm{L}^{-1}\end{array}$ & $\begin{array}{c}{[\mathrm{Nu}]_{\mathrm{eff}}} \\
/ \mathrm{mol} \mathrm{L}^{-1}\end{array}$ & $\begin{array}{c}{\left[\mathrm{OH}^{-}\right]} \\
/ \mathrm{mol} \mathrm{L}^{-1}\end{array}$ & {$[\mathrm{Nu}]_{\mathrm{eff}} /[\mathrm{El}]_{0}$} & $\begin{array}{l}k_{\mathrm{obs}} \\
/ \mathrm{s}^{-1}\end{array}$ & $\begin{array}{c}k_{1 \Psi, \mathrm{OH}^{-}} \\
/ \mathrm{s}^{-1}\end{array}$ & $\begin{array}{l}k_{1 \Psi} \\
/ s^{-1}\end{array}$ \\
\hline $\mathrm{fb} 47.2$ & $5.36 \times 10^{-5}$ & $4.21 \times 10^{-3}$ & $3.77 \times 10^{-3}$ & $4.45 \times 10^{-4}$ & 70 & $1.14 \times 10^{2}$ & $1.05 \times 10^{-2}$ & $1.14 \times 10^{2}$ \\
\hline $\mathrm{fb} 47.3$ & $5.36 \times 10^{-5}$ & $1.68 \times 10^{-3}$ & $1.41 \times 10^{-3}$ & $2.72 \times 10^{-4}$ & 26 & 42.8 & $6.42 \times 10^{-3}$ & 42.8 \\
\hline $\mathrm{fb} 47.4$ & $5.36 \times 10^{-5}$ & $8.42 \times 10^{-4}$ & $6.56 \times 10^{-4}$ & $1.86 \times 10^{-4}$ & 12 & 19.1 & $4.38 \times 10^{-3}$ & 19.1 \\
\hline \multicolumn{5}{|c|}{$k_{2, \mathrm{~N}}=3.04 \times 10^{4} \mathrm{M}^{-1} \mathrm{~s}^{-1}$} & 120 & & & \\
\hline \multicolumn{5}{|c|}{$k_{2, \mathrm{OH}^{-}}=23.6 \mathrm{M}^{-1} \mathrm{~s}^{-1}$} & is 80 & & & \\
\hline \multirow{3}{*}{\multicolumn{2}{|c|}{$\mathrm{p} K_{\mathrm{B}}=4.28$}} & & & & 40 & & & \\
\hline & & & & & 0.000 & 0.001 & 0.002 & 0.004 \\
\hline & & & & & \multicolumn{4}{|c|}{$[\text { Nucleophile }]_{\text {eff }} / \mathrm{mol} \mathrm{L}^{-1}$} \\
\hline
\end{tabular}

29.1.5. Reaction of Piperazine (1zz) with (ind) ${ }_{2} \mathrm{CH}^{+} \mathrm{BF}_{4}{ }^{-}$(at $20^{\circ} \mathrm{C}$, cosolvent 0.4 vol- $\% \mathrm{CH}_{3} \mathrm{CN}$, stopped-flow, detection at $618 \mathrm{~nm}$ )

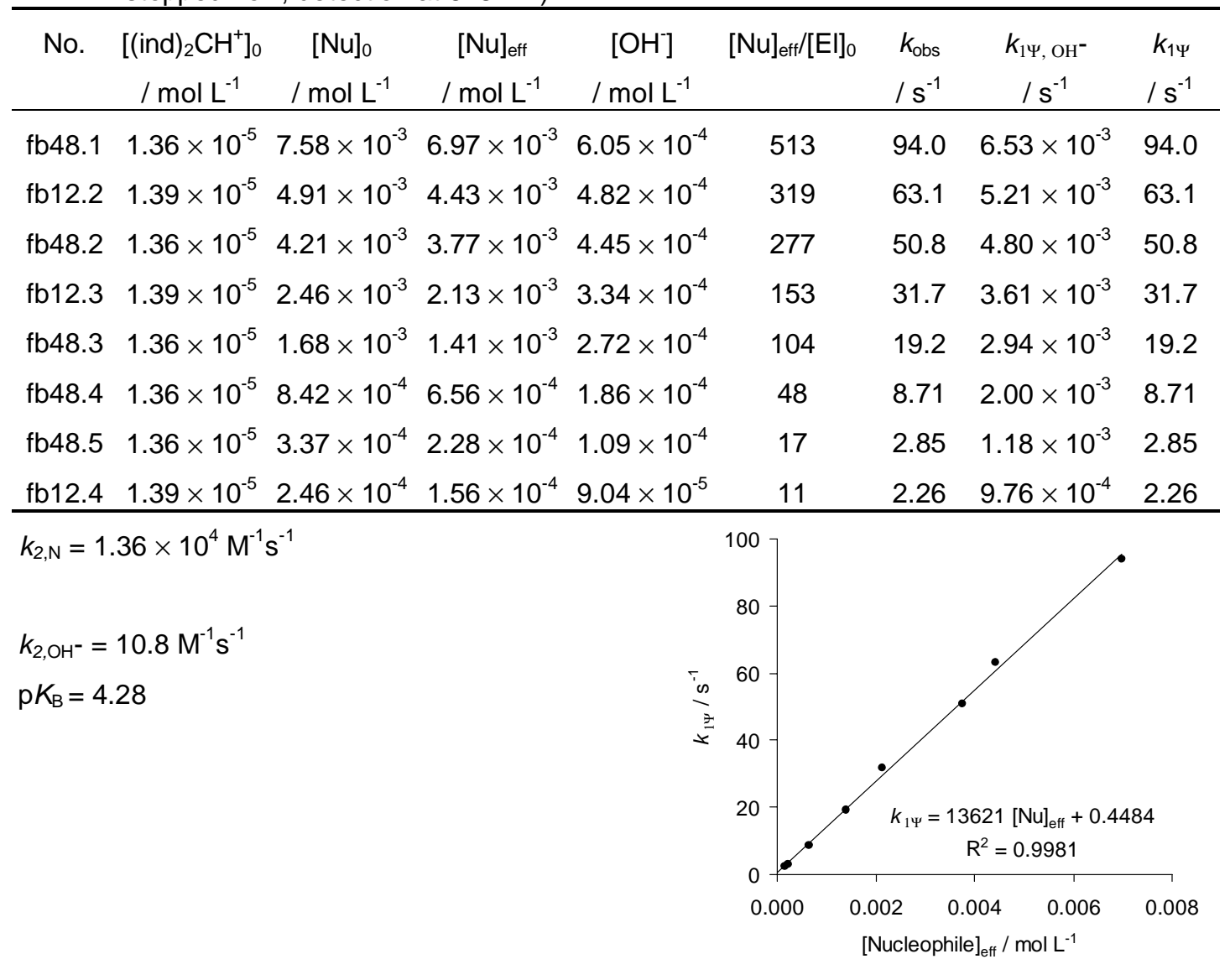


29.1.6. Reaction of Piperazine (1zz) with (jul) ${ }_{2} \mathrm{CH}^{+} \mathrm{BF}_{4}{ }^{-}$(at $20^{\circ} \mathrm{C}$, cosolvent 0.4 vol- $\% \mathrm{CH}_{3} \mathrm{CN}$, stopped-flow, detection at $634 \mathrm{~nm}$ )

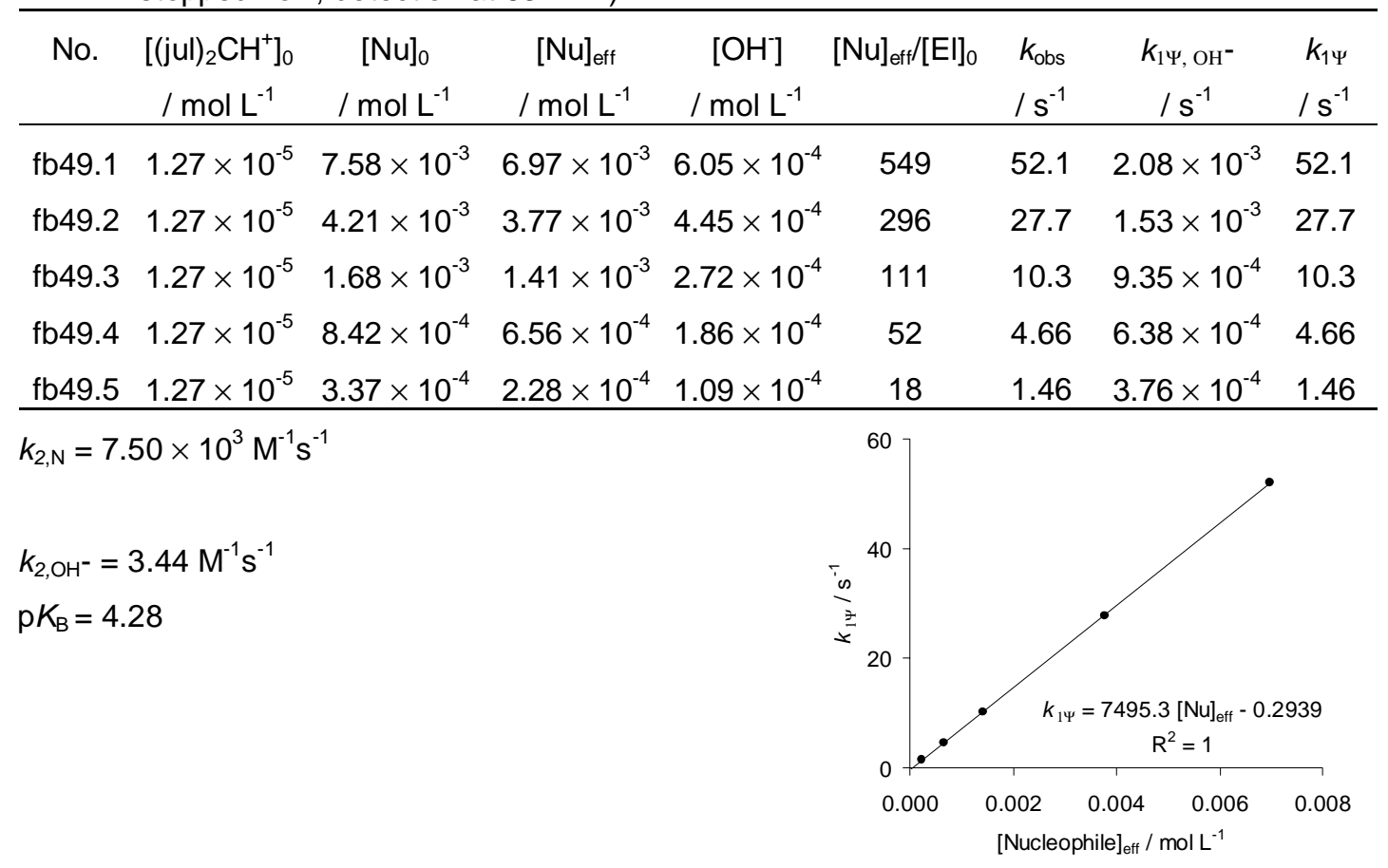

29.1.7. Reaction of Piperazine (1zz) with (lil) ${ }_{2} \mathrm{CH}^{+} \mathrm{BF}_{4}^{-}$(at $20^{\circ} \mathrm{C}$, cosolvent 0.4 vol- $\% \mathrm{CH}_{3} \mathrm{CN}$, stopped-flow, detection at $604 \mathrm{~nm}$ )

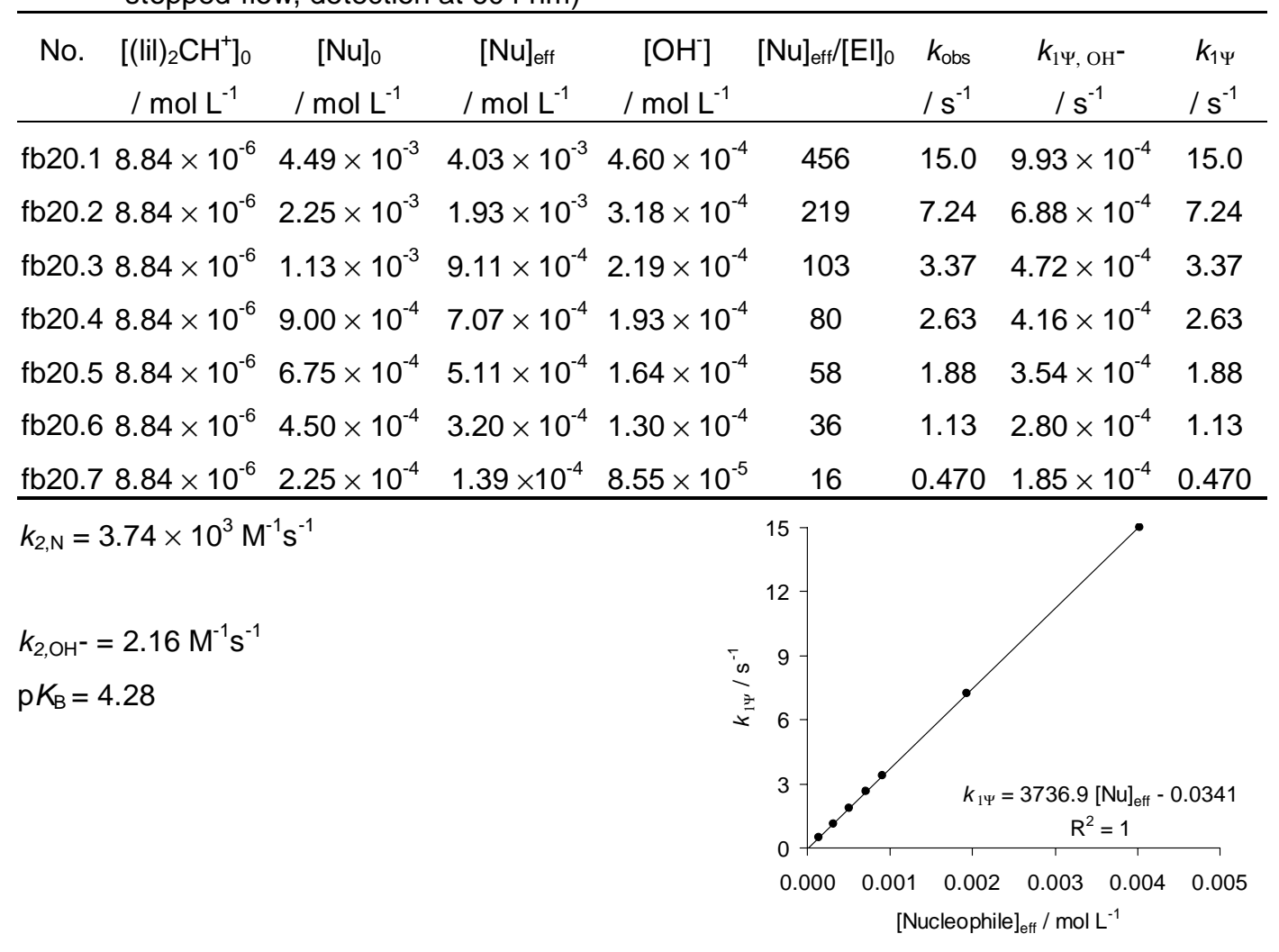


29.2. Reactivity parameters in water: $N=17.22 ; s=0.50$

\begin{tabular}{ccc}
\hline Reference electrophile & $E$ parameter & $k_{2, \mathrm{~N}}\left(20^{\circ} \mathrm{C}\right) / \mathrm{M}^{-1} \mathrm{~s}^{-1}$ \\
\hline (mor $)_{2} \mathrm{CH}^{+}$ & -5.53 & $6.10 \times 10^{5}$ \\
$(\text { dma })_{2} \mathrm{CH}^{+}$ & -7.02 & $1.27 \times 10^{5}$ \\
$(\mathrm{pyr})_{2} \mathrm{CH}^{+}$ & -7.69 & $5.34 \times 10^{4}$ \\
$(\text { thq })_{2} \mathrm{CH}^{+}$ & -8.22 & $3.04 \times 10^{4}$ \\
$(\text { ind })_{2} \mathrm{CH}^{+}$ & -8.76 & $1.36 \times 10^{4}$ \\
$(\mathrm{jul})_{2} \mathrm{CH}^{+}$ & -9.45 & $7.48 \times 10^{3}$ \\
$(\mathrm{lil})_{2} \mathrm{CH}^{+}$ & -10.04 & $3.74 \times 10^{3}$ \\
\hline
\end{tabular}

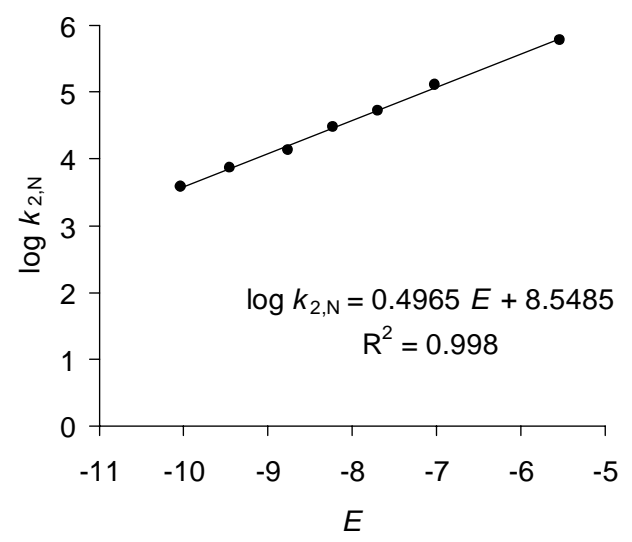

\section{Literature}

${ }^{1}$ Heo, C. K. M.; Bunting, J. W. J. Chem. Soc., Perkin Trans. 2 1994, 2279-2290.

${ }^{2}$ Minegishi, S.; Mayr, H. J. Am. Chem. Soc. 2003, 125, 286-295.

${ }^{3}$ Virtanen, P. O. I.; Korhonen, R. Acta Chem. Scand. 1973, 27, 2650-2654.

${ }^{4}$ Minegishi, S.; Kobayashi, S.; Mayr, H. J. Am. Chem. Soc. 2004, 126, 5174-5181.

${ }^{5}$ Phan, T. B.; Mayr, H. Can. J. Chem. 2005, 83, 1554-1560.

${ }^{6}$ Garcia-Rio, L.; Iglesias, E.; Leis, J. R.; Peña, M. E.; Rios A. J. Chem. Soc., Perkin Trans. 21993, 29-

37.

${ }^{7}$ Brotzel, F.; Mayr, H.; unpublished results

${ }^{8}$ Munro, A. P.; Williams, D. L. H. J. Chem. Soc., Perkin Trans. 2 1999, 1989-1993.

${ }^{9}$ Um, I.-H.; Lee, E.-J.; Seok, J.-A.; Kim K.-H. J. Org. Chem. 2005, 70, 7530-7536.

${ }^{10}$ Edsall, J. T.; Blanchard, M. H. J. Am. Chem. Soc. 1933, 55, 2337-2353.

${ }^{11}$ Bunting, J. W.; Stefanidis, D. J. Am. Chem. Soc. 1990, 112, 779-786.

${ }^{12}$ Chebotarev, A. N.; Kachan, S. V. Russ. J. Phys. Chem. 1991, 65, 360-362.

${ }^{13}$ CRC Handbook of Chemistry and Physics, CRC Press, Boca Raton, 84 ${ }^{\text {th }}$ ed., 2003. 